\title{
SILVER I-MOTIFS AND THEIR ANTIBACTERIAL ACTIVITY
}

BY

\section{JESSICA BRATT}

VICTORIA UNIVERSITY OF WELLINGTON

2020

A thesis submitted in fulfilment of the requirements for the degree of Master of

Biomedical Science

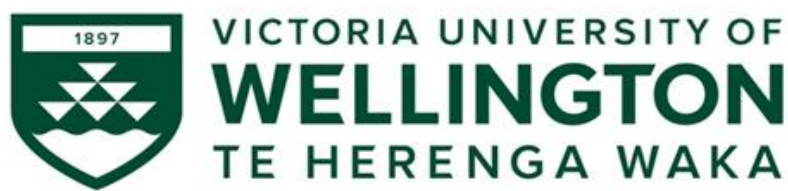





\begin{abstract}
The spread of antibiotic resistance and the emergence of multi-drug resistant bacteria is a major threat to public health. This study investigated a unique cytosine rich DNA structure, the i-Motif to deliver soluble $\mathrm{Ag}^{+}$as a novel antimicrobial agent (AgiMs). AgiMs were evaluated in vitro against P. aeruginosa and A. baumannii strains. AgiMs displayed significant antibacterial activity against both $P$. aeruginosa and $A$. baumannii (median MIC: $0.875 \mu \mathrm{M}$ and $0.75 \mu \mathrm{M}$, respectively) by rapid, bactericidal and concentrationdependent effect. Low concentrations of AgiMs showed efficacy against PA01 20-h biofilms, resulting in 57\% reduction in biomass (5 x MIC). A single dose of AgiMs extended survival of $G$. Mellonella larvae, with the therapeutic benefit paralleled in the reduction of internal bacterial load. Synergistic interactions were observed with the combination of AgiMs and tobramycin, a common antibiotic used to treat P. aeruginosa infections; indicating the potential for AgiMs to reinstate the potency of current antibiotics. This silver-based agent might be an alternative to the failing antibiotic regimes for MDR resistant infections. Further in vitro and in vivo studies are warranted to confirm the therapeutic potential.
\end{abstract}




\section{ACKNOWLEDGEMENTS}

First and foremost, I want to thank my supervisor, Dr Darren Day, who has guided and inspired me throughout the journey of this thesis with his passion for science and entertaining goat wrangling tales.

Jen, this thesis would not be possible without you. Thank you for welcoming me onto your project, and for the constant guidance and support you have given me without hesitation (not to mention cakes and formatting help!). You have been a constant inspiration to me during my time at Victoria, and I truly treasure our friendship. You will always be our Alpha.

Daniel, my fellow mischief-maker, your friendship and support have made my experience unforgettable and you will always hold a special place in my heart. From sharing lollies at our desks to long discussions in your office or on the bus home, and far too many Dad jokes - thanks for keeping me sane, annoyed (only once) and entertained (always).

Varun, your presence in the lab, whether for help or hilarity, was always welcome. You have guided me throughout my time at Victoria and I will always remember your great advice and sass.

A big thanks to the DJD lab - thank you for your support. I will always cherish our hot chocolate times and Covid-quizzes. I never thought postgrad life would involve so many dishes and autoclaving rubbish - but we got through it together!

To my part-one comrades, especially Aanchal, Janine, Sharna and Katie, thank you for the friendship and laughs you have given me throughout this experience. Coffee breaks, treat runs and 'office chats' helped keep me sane and smiling. We made it!

To my friends - thank you for putting up with me over the past two years. Your encouragement and love has been unwavering and I couldn't have done it without you. I love you all so much.

To my family; Mum, Dad, Ros, Sim, Trini and Electra. Thank you for your constant love and support. I would not have succeeded without your encouragement and I am so lucky to have you all.

And finally, to all of the SBS family, thanks for the memories xxx 


\section{TABLE OF CONTENTS}

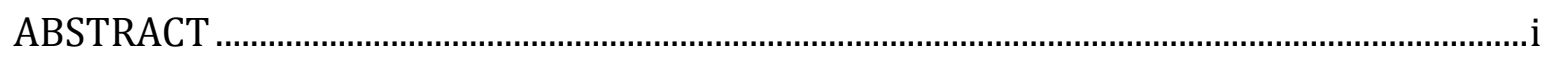

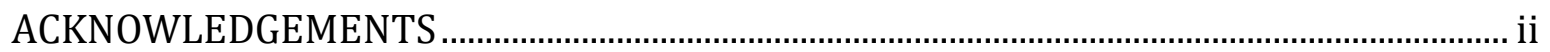

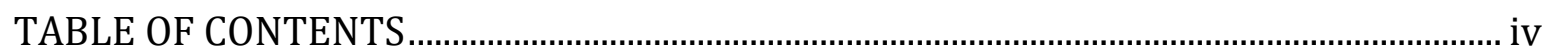

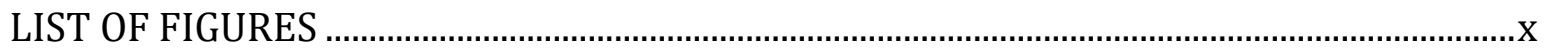

LIST OF TABLES

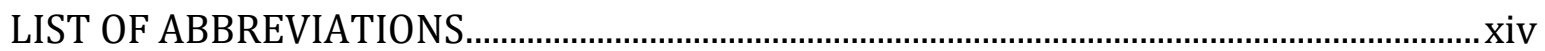

CHAPTER 1. INTRODUCTION

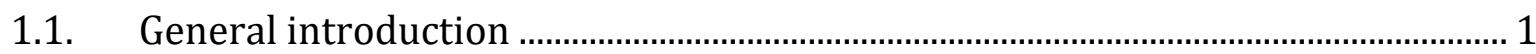

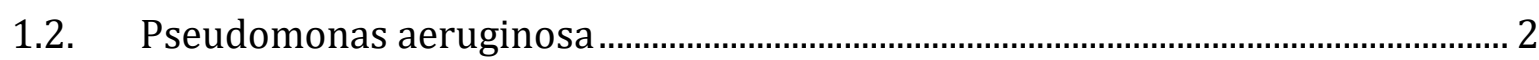

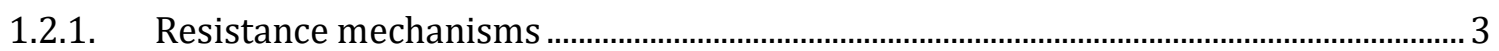

1.2.1.1. Intrinsic resistance mechanisms ………................................................................ 4

1.2.1.2. Acquired resistance mechanisms............................................................................. 5

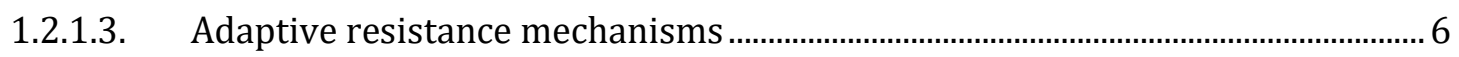

1.2.1.4. Biofilms and persister cell population ....................................................................... 6

1.2.1.5. Virulence factors ........................................................................................................ 8

1.3. Current therapeutic options and emerging technologies ......................................... 9

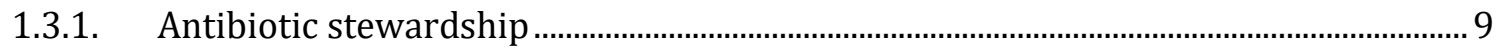

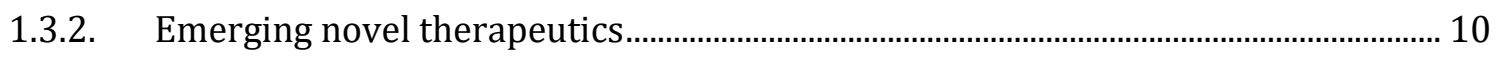

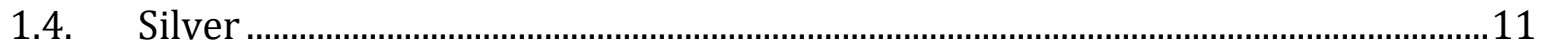

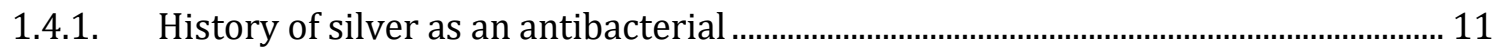

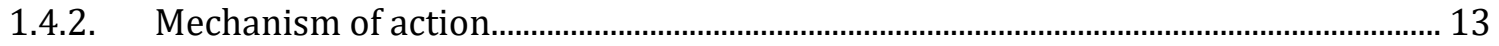

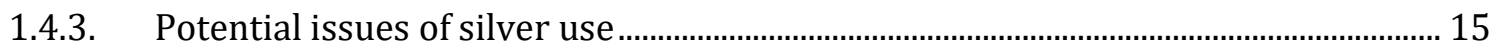




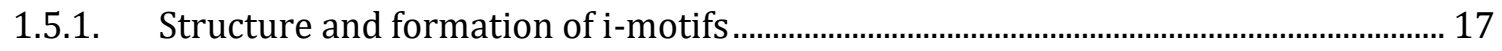

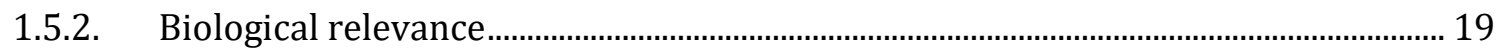

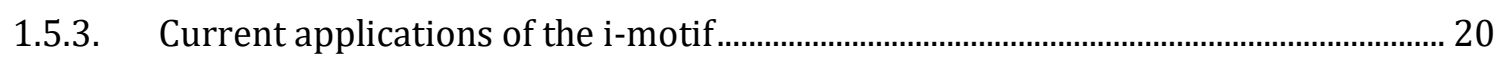

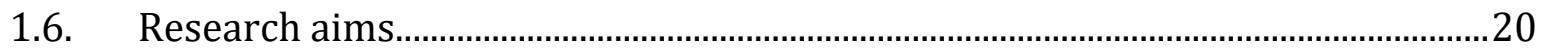

CHAPTER 2. MATERIALS AND METHODS …....................................................................23

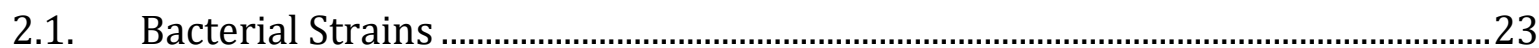

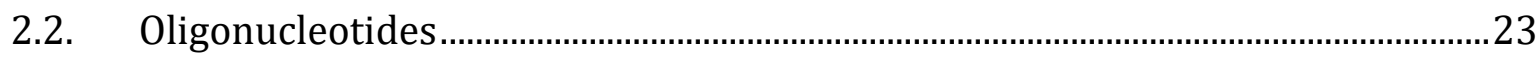

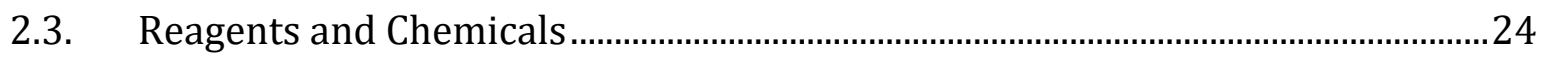

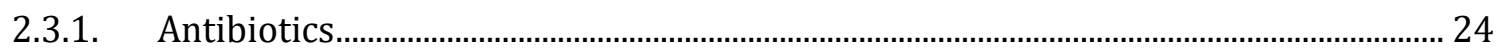

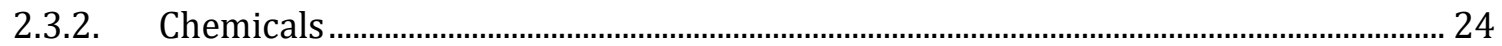

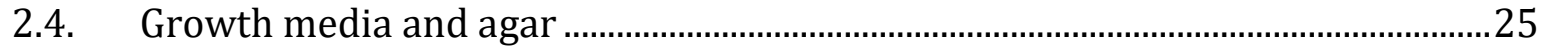

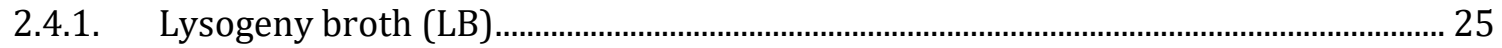

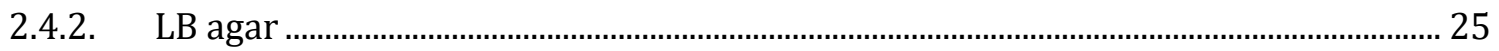

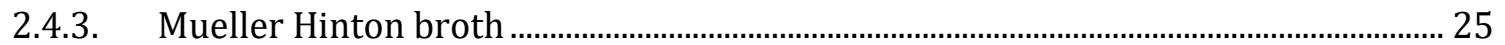

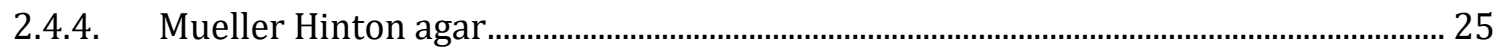

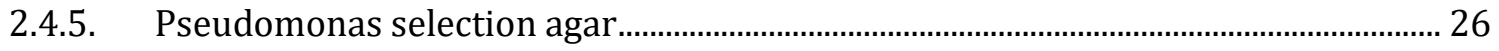

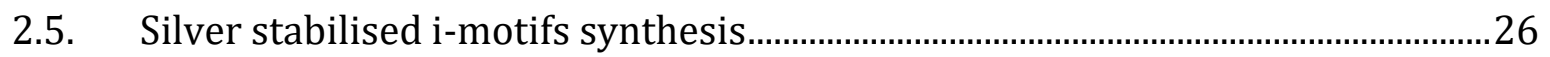

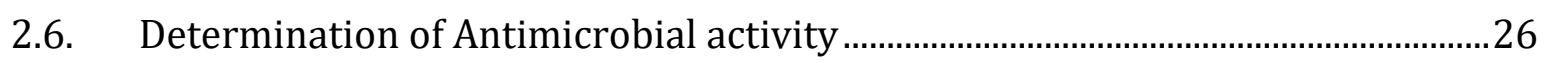

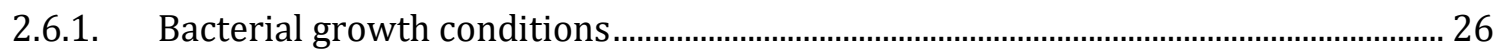

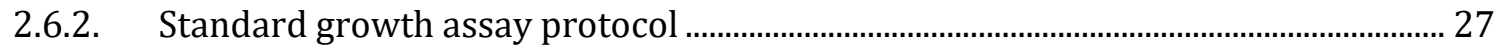

2.6.3. Optical density by UV spectroscopy............................................................................... 27

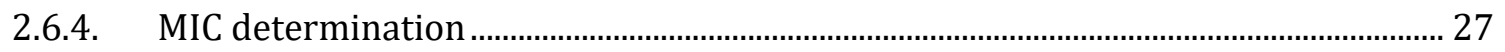

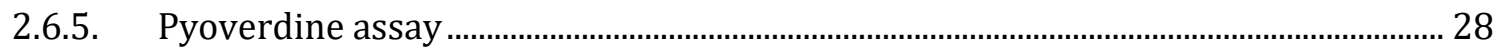

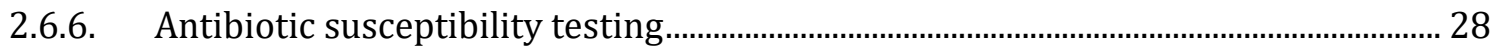




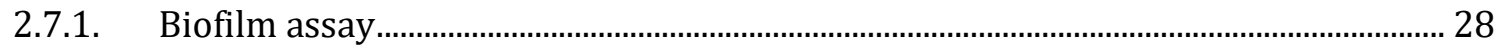

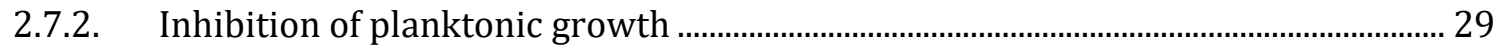

2.7.3. Biofilm biomass determination .................................................................................... 29

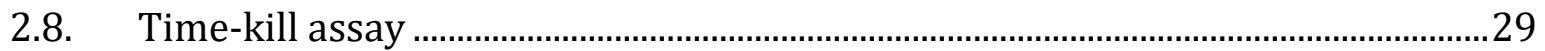

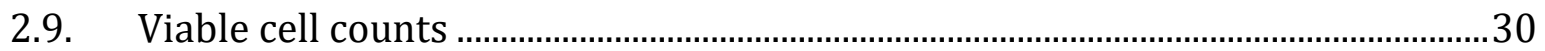

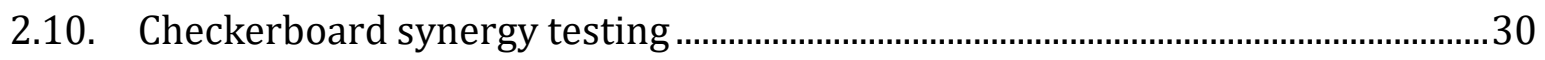

2.10.1. FIC determination for synergy testing ....................................................................... 31

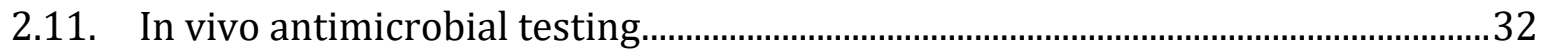

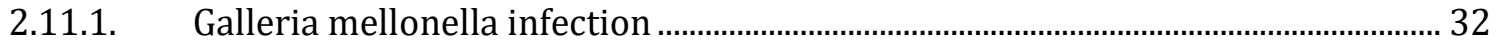

2.11.2. Determination of bacterial load in the haemolymph................................................. 33

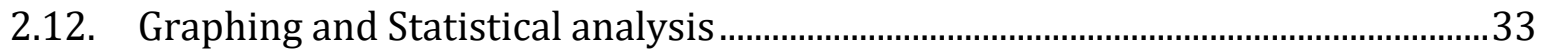

CHAPTER 3. OPTIMISATION OF AGIM FOR ANTIBACTERIAL ACTIVITY ......................35

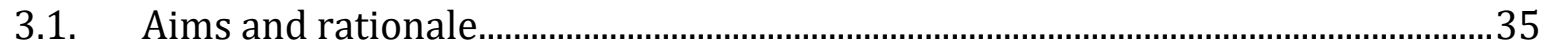

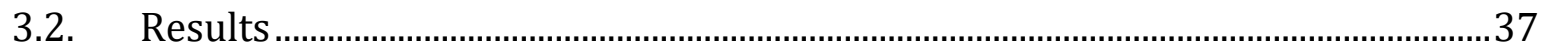

3.2.1. AgiMs exhibit antibacterial activity ……….................................................................... 37

3.2.2. The poly-C region of AgiMs associated with antimicrobial potency .......................... 44

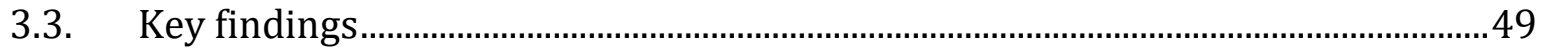

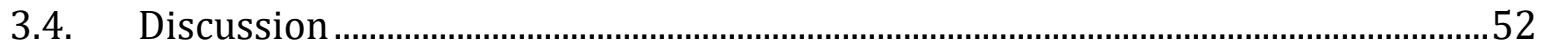

CHAPTER 4. INVESTIGATION OF AGIMS IN VITRO ANTIBACTERIAL ACTIVITY ......55

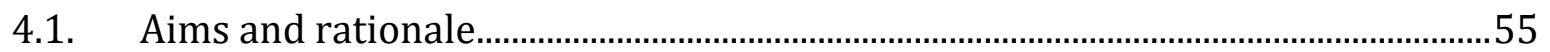

4.2. Bacterial strains antibiotic susceptibilities..........................................................

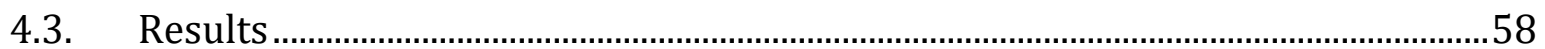


4.3.1. AgiM exhibit dose-dependent killing of P. aeruginosa .................................................... 58

4.3.2. AgiM exhibit dose-dependent killing of A. baumannii ..................................................... 62

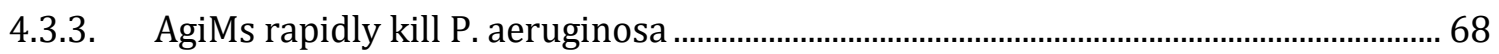

4.3.4. AgiMs inhibit the production of pyoverdine …………...................................................... 73

4.3.5. AgiMs are not substrates for efflux pumps ……............................................................. 77

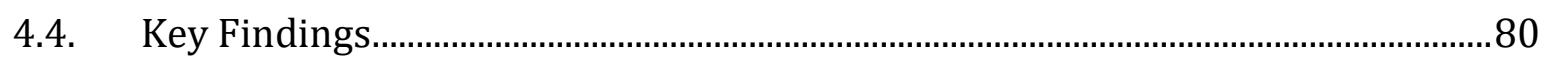

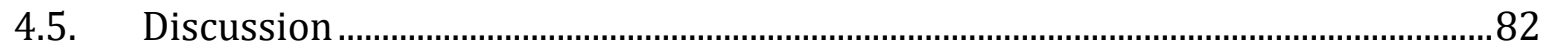

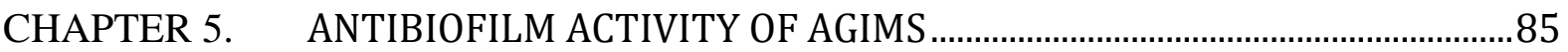

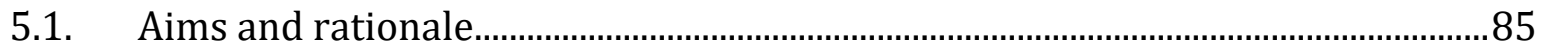

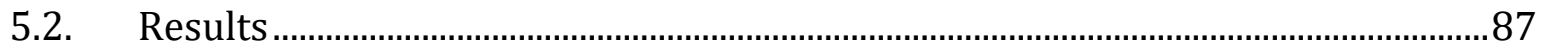

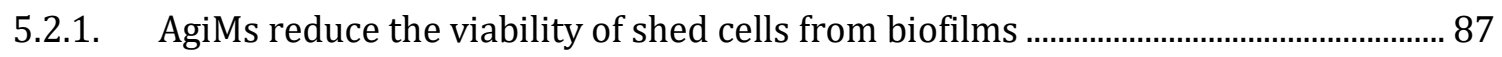

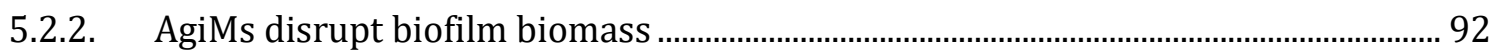

5.2.3. AgiM reduces the viability of 20-h biofilms...................................................................... 97

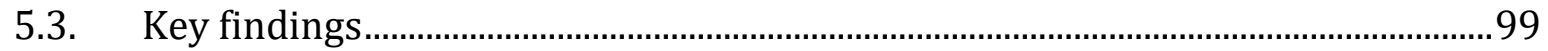

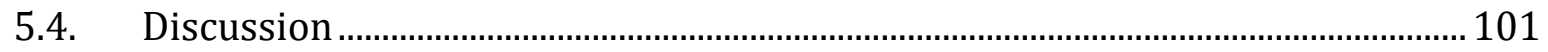

5.4.1. Evaluation of experimental design and technique ...................................................103

5.4.1.1. Closed v open system of biofilm growth ............................................................ 103

5.4.1.2. Evaluation of biofilm biomass .............................................................................. 104

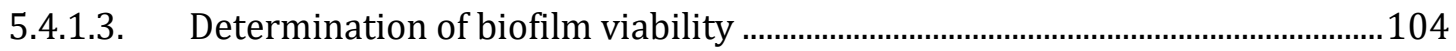

5.4.1.4. Limitations of in vitro biofilm susceptibility testing ............................................ 105

CHAPTER 6. AGIMS AND SYNERGY AGAINST P. AERUGINOSA..................................... 107

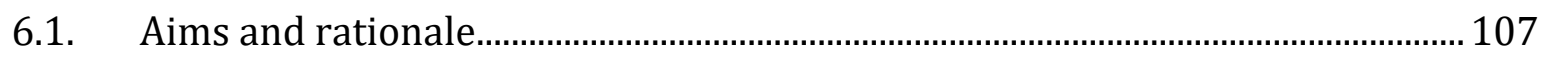

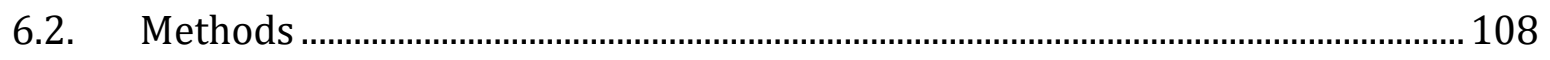

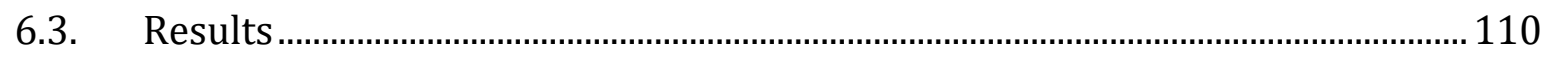

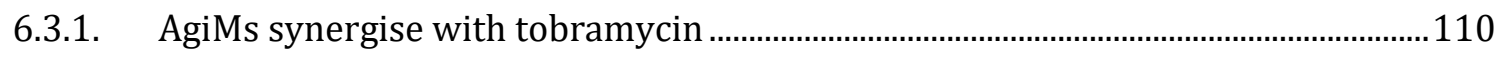


6.5. Discussion

CHAPTER 7. INVESTIGATION OF IN VIVO ACTIVITY OF AGIMS

7.1. Aims and rationale

7.2. Results

7.2.1. G. Mellonella are highly susceptible to infection by P. aeruginosa . .118

7.2.2. Treatment with AgiMs extends survival of G. mellonella larvae after infection with P. aeruginosa

7.2.2.1. AgiM treatment of P. aeruginosa PAO1 exhibits a dose-dependent increase in larval survival.

7.2.2.2. AgiM treatment of P. aeruginosa clinical isolates exhibits a dose-dependent increase in larval survival

7.2.3. Enhanced larval survival after treatment of AgiM results in a parallel reduction in the number of viable CFU in the larval haemolymph

7.2.3.1. Bacterial load observed at 12-h and 24-h post-infection....................................131

7.2.3.2. The number of viable CFU in haemolymph at the onset of mortality 134

7.2.4. AgiMs in combination with tobramycin extend survival of G. mellonella 138

7.3. Key findings 139

7.4. Discussion 141

CHAPTER 8. GENERAL DISCUSSION 145

8.1. Research aims

8.2. Research outcomes

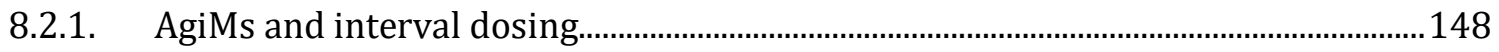

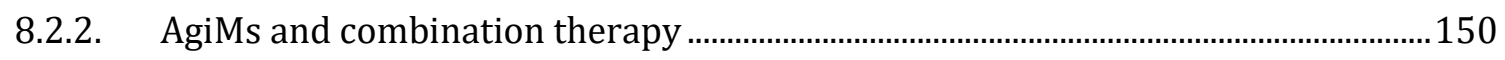

8.3. Considerations for AgiMs as a potential therapeutic ........................................... 150 


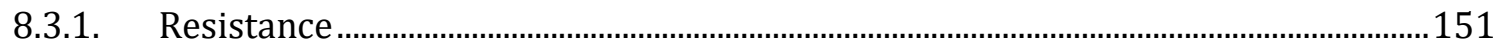

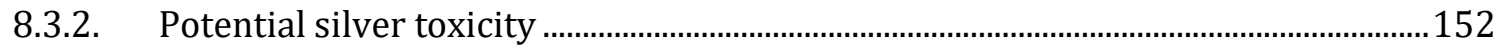

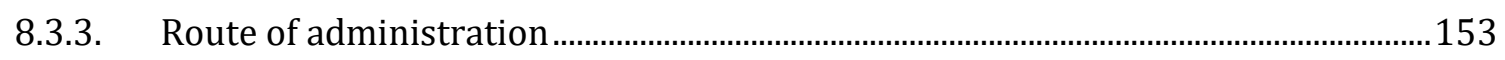

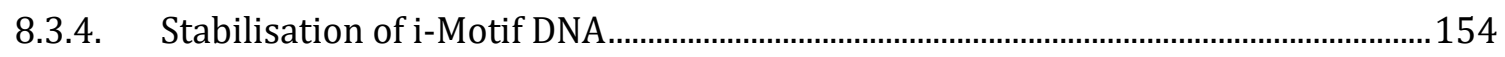

8.3.5. The commercial viability of AgiM …………............................................................ 155

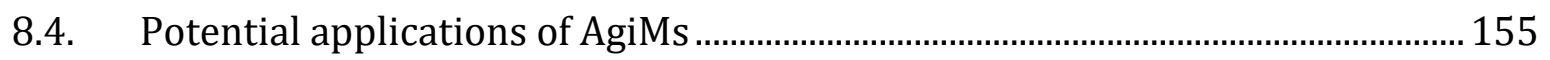

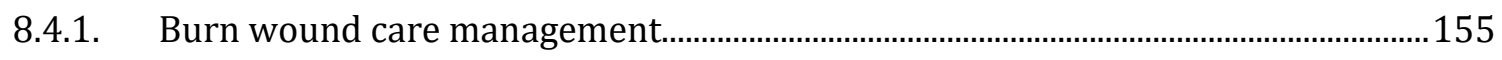

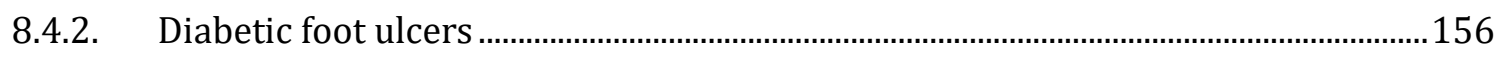

8.4.3. Cystic fibrosis and respiratory tract infections...........................................................156

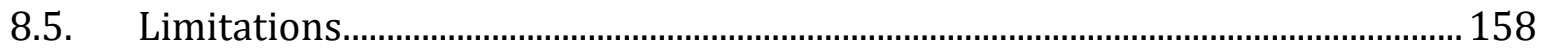

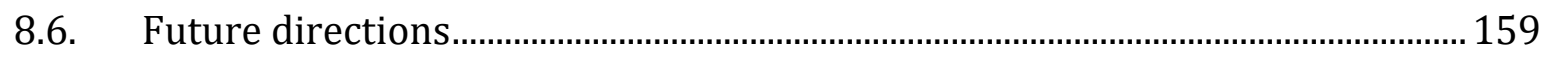

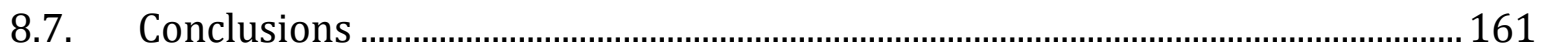

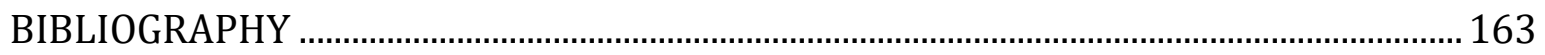

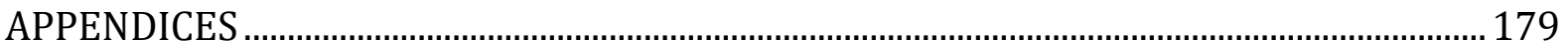




\section{LIST OF FIGURES}

Figure 1.1 Resistance mechanisms of Gram-negative bacteria P. aeruginosa...................... 4

Figure 1.2 Biofilm maturation process.................................................................................... 7

Figure 1.3 Schematic of the antibacterial mechanisms of Ag+ in bacteria.........................15

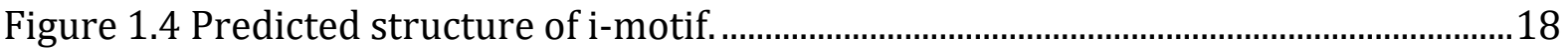

Figure 2.1 Schematic of checkerboard synergy plate layout in 96-well micro-titre plate.

Figure 3.1 Comparison of 6:1 ratio with excess $\mathrm{Ag}^{+}$ratio in Full i-motif 2..........................40

Figure 3.2 Comparison of 6:1 Ag:DNA molar ratio with 10:1 Ag:DNA in Half i-motif 2...42

Figure 3.3 The number of $\mathrm{C}-\mathrm{C}+\mathrm{bp}$ is proportional to the antibacterial activity of AgiMs

Figure 3.4 Dose-response curves demonstrate AgiM exhibit strong antibacterial activity.

Figure 3.5 Antibacterial activity: 48

Figure 3.6 Comparison of iMotif sequence $\mathrm{C}-\mathrm{C}+$ pair number to corresponding bacterial growth inhibiton. .50

Figure 3.7 Schematic of proposed binding mechanism of $\mathrm{Ag}^{+}$in AgiM..................................52

Figure 4.1a-c Dose-dependent antibacterial effect of AgiM HiM3.

Figure 4.2 Dose-response curves of AgiM HiM3 antibacterial activity show low IC50

values

Figure 4.3 Dose-dependent antibacterial activity of AgiM HiM3 against A. baumannii...65

Figure 4.4 Dose-response curves of AgiM HiM3 antibacterial activity show low IC $_{50}$

values against $\mathrm{A}$. baumannii. .66

Figure 4.5 Time-kill curves demonstrate AgiM killing of inoculum. 
Figure 4.6 AgiMs rapidly kill P. aeruginosa strains.

Figure 4.7 Subinhibitory concentrations of AgiM reduce siderophore pyoverdine..........76

Figure 4.8 AgiM are not substrates for P. aeruginosa efflux pumps........................................79

Figure 5.1 Proposed antibacterial activity of AgiMs on established biofilms. ...................86

Figure 5.2 High concentrations of AgiM treatment reduce planktonic shedding from 20-

$\mathrm{h}$ and 44-h biofilms. .90

Figure 5.3 Comparison of highest concentration AgiM treatment ( $5 \mu \mathrm{M})$ with untreated control on planktonic cells shed from 20 -h and 40 -h biofilms. 91

Figure 5.4 High concentrations of AgiM treatment disrupt 20-h and 44-h biofilms .94

Figure 5.5 Effects of $5 \mu \mathrm{M}$ AgiM on 20-h and 44-h biofilms produced by P. aeruginosa..96

Figure 5.6 The effect of AgiM on 20-h and 44-h PA01 biofilm viability .98

Figure 6.1 Schematic of Isobologram graph. 109

Figure 6.2 a-d: Isobolograms confirm synergistic and additive interactions between AgiM and tobramycin in four strains of P. aeruginosa.

Figure 7.1 Effect of varying inoculum doses of $P$. aeruginosa strains on the survival of $G$. mellonella larvae 120

Figure 7.2 Effect of AgiM treatment on the survival of G. Mellonella larvae infected with PA01 strain of P. aeruginosa 124 Figure 7.3 Effect of AgiM treatment on the survival of G. Mellonella larvae infected with clinical isolates of P. aeruginosa. 128

Figure 7.4 Survival proportion of larvae at 4-h after onset of mortality 129 Figure 7.5 The effect of AgiMs treatment on survival of Galleria mellonella and the corresponding internal bacterial load of PA01 133

Figure 7.6 The effect of AgiM treatment on survival and internal larval burden of P. aeruginosa strains at onset of mortality 136 
Figure 7.7 Effect of combined treatment of AgiM/tobramycin on the survival of G.

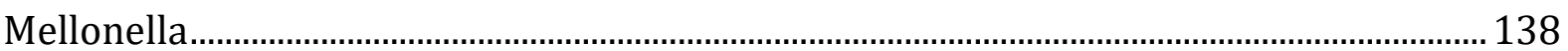

Figure 8.1 Proposed effect of 'interval dosing' of AgiMs on P. aeruginosa mature biofilms 


\section{LIST OF TABLES}

Table 2-1 Bacterial strains used in the study. .23

Table 2-2 Oligonucleotide sequences used in this study. .23

Table 2-3 Antibiotic agents used in this thesis .24

Table 2-4 FIC interpretations for evaluation of synergistic activity 32

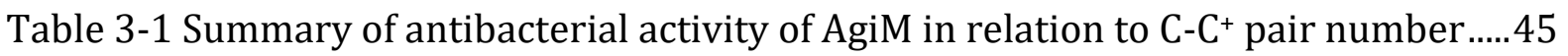

Table 4-1 Antibiotic susceptibility of P. aeruginosa strains used in this study. .56

Table 4-2 Antibiotic susceptibilities of A. baumannii strains used in this study .57

Table 4-3 Summary of AgiM HiM3 activity against Gram-negative bacteria P. aeruginosa and A. baumannii 80

Table 6-1 in vitro sensitivities of P. aeruginosa strains to antimicrobial agents AgiM and tobramycin 110

Table 6-2 FIC calculations and interpretations for all strains of P. aeruginosa determined from checkerboard assay 111

Table 7-1: Summary of inoculum size required to achieve $\geq 80 \%$ death of larvae within 24-48 h post-infection in different strains of $\mathrm{P}$. aeruginosa. 119 Table 7-2 The effect of AgiM treatment on the viability of $P$. aeruginosa strains, and the corresponding log reduction in internal larval burden 135 


\section{LIST OF ABBREVIATIONS}

\begin{tabular}{ll} 
Ag & silver \\
AgiM & silver i-Motif \\
AgNC & silver nanocluster \\
AgNP & silver nanoparticle \\
AgNO3 & silver nitrate \\
AgSD & silver sulfadiazine \\
AUC & area under the curve \\
Bp & base pair \\
C & cytosine \\
CD & circular dichromism \\
CF & cystic fibrosis \\
CFU & colony forming unit \\
CI & confidence interval \\
ddH20 & double distilled water \\
DNA & Deoxyribonucleic Acid \\
EDTA & Ethylenediaminetetraacetic acid \\
EtOh & Ethanol \\
FIC & fractional inhibitory concentration \\
IC50 & half maximal inhibitory concentration \\
LB & lysogeny broth \\
MES & 2-(N-morpholino)ethanesulfonic acid \\
MDR & multi drug resistance \\
MIC & minimum inhibitory concentration \\
Nm & nanometre \\
OD & optical density \\
PBS & phosphate-buffered saline \\
RND & resistance nodulation division \\
ROS & reactive oxygen species \\
RT & room temperature \\
SD & standard deviation \\
SEM & standard error of the mean \\
ssDNA & single-stranded DNA \\
QS & quorum sensing \\
& \\
\hline &
\end{tabular}




\section{CHAPTER 1. INTRODUCTION}

\subsection{General introduction}

The emergence and exponential rise of antibiotic resistance mark a sentinel event in modern medicine. The increasing rate of resistant pathogens exceeds that of new drug discovery with no new major classes of antibiotics introduced between 1962 and 2000, threatening a new post-antibiotic reality (1). During the last few decades, microorganisms have evolved to gain exceptional resistance, with some showing insensitivities to all known antibiotics. The consequences of this rise in resistance are seen in the increase of chronic nosocomial infections with higher rates of mortality and morbidity. These persistent infections are a significant burden on healthcare systems and also economically. The World Health Organisation (WHO) announced that failure to act of the issue of MDR bacteria would lead to the loss of 10 million lives annually by 2050, at a cost to the world economy of 100 trillion USD (2).

The notoriety of resistant nosocomial microorganisms is exemplified by the ESKAPE pathogens; an acronym for a collection of Gram-negative and Gram-positive species made up of Enterococcus faecium, Staphylococcus aureus, Klebsiella pneumoniae, Acinetobacter baumannii, Pseudomonas aeruginosa, and Enterobacter (2)(3). The ESKAPE pathogens are the leading cause of nosocomial infections globally and exhibit high levels of resistance, enabling them to escape the effects of antibiotic treatment. The bacteria involved in this coterie represent the archetypal microorganism, demonstrating high levels of pathogenesis and resistance that allow them to be so successful in the hospital environment. 
The rise of multi-drug resistant bacteria highlights the urgent need for new antibacterial compounds with novel delivery and targeting strategies to avoid the development of resistance. In this study, silver-stabilised i-motifs (AgiMs) were investigated against $P$. aeruginosa to evaluate their antibacterial activity. These unique cytosine rich DNA secondary structures contain silver ions, which have well-established toxicity towards microbes (4). This thesis seeks to determine the viability of AgiMs as a novel therapeutic against the rising threat of resistant bacteria. Chapter 3 describes the optimisation of AgiMs to achieve antibacterial activity. Investigation of the antibacterial activity of AgiMs was continued in chapter 4, which evaluates the effect of AgiMs treatment in planktonic cultures and chapter 5, which explores the ability of AgiMs to disrupt and eradicate established biofilms. The potential synergistic interactions of AgiMs in combination with aminoglycosides to enhance therapeutic efficacy at low doses was explored in chapter 6. Finally, the antibacterial activity of AgiMs was investigated using an invertebrate model of infection.

\subsection{Pseudomonas aeruginosa}

In this era of antibiotic resistance, Pseudomonas aeruginosa represents one of the most concerning pathogens involved in antibiotic resistance and therefore, was selected as the test pathogen for this thesis. The Gram-negative bacterium P. aeruginosa is a ubiquitous environmental bacterium typically found in soil and aqueous environments that has emerged as a major opportunistic human pathogen over the past century. This clinically significant pathogen causes a variety of infections, including urinary, respiratory, and other soft-tissue infections, particularly in immunocompromised patients such as burn victims and individuals with cancer or cystic fibrosis resulting in severe infections with 
high mortality and morbidity rates (5). Due to its adaptability and affinity for aqueous environments, $P$. aeruginosa is one of the most common culprits of respiratory infections and is associated with the chronic colonisation of the lungs of cystic fibrosis patients that ultimately leads to pulmonary failure and death (6).

Antimicrobial resistance is a substantial problem in the treatment of $P$. aeruginosa due to the inherent insensitivity of the pathogen to a wide variety of antimicrobial drugs and its rapid acquisition of additional resistance mechanisms (4).

\subsubsection{Resistance mechanisms}

P. aeruginosa is associated with both acute and chronic infections that are extremely difficult to eradicate due to the precedence of multi-drug resistant strains. Multi-drug resistance (MDR) is defined as the insensitivity of a microorganism to three or more of the following antimicrobial classes: cephalosporins, carbapenems ampicillin-sulbactam, fluoroquinolones and aminoglycosides (7). These MDR microorganisms render current antimicrobial treatments ineffective, leading to chronic infections with increased morbidity.

The exceptional resistance of $P$. aeruginosa is attributed to multiple resistance mechanisms conferring resistance to a variety of antibacterial compounds. These mechanisms include intrinsic, adaptive and acquired resistance, which are summarised in figure 1 and discussed below. 


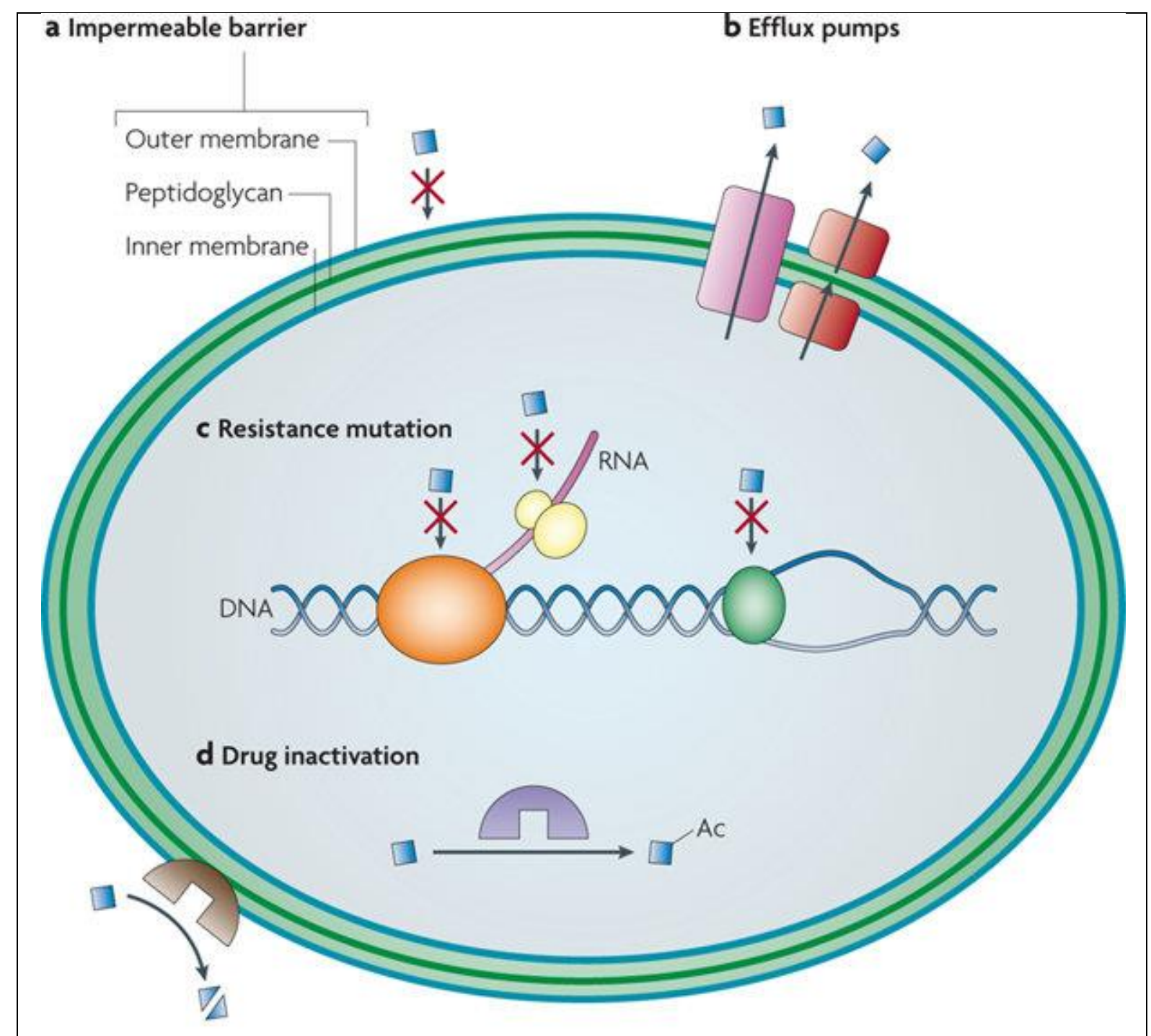

Figure 1.1 Resistance mechanisms of Gram-negative bacteria P. aeruginosa.

A variety of resistance mechanisms are involved in the exceptional resistance of P. aeruginosa infections. (a) The impermeable barrier is due to the intrinsic resistance to antibiotics (blue squares) due to low permeability of the outer membrane (b) Multi-drug efflux pumps extrude antibiotics from the cell. The RND efflux pump (pink) can pump antibiotics directly outside of the cell while the major facilitator superfamily (red) secretes antibiotic into the periplasm (c) resistance mutations, these mutations modify the target protein, for example by disabling the antibiotic-binding site but leaving the cellular function of the protein intact. An example is a mutation in gyrase (green) that causes resistance to fluoroquinolones and (d) drug inactivation can occur by covalent modification of the antibiotic. An example is the degradation of $\beta$-lactam antibiotics catalysed by $\beta$-lactamases (brown). Reprinted from Nature Reviews Microbiology, Heather K. Allen et al., Call of the wild: antibiotic resistance genes in natural environments, March 1, 2010, Copyright (C) 2010, Springer Nature

\subsubsection{Intrinsic resistance mechanisms}

Intrinsic resistance is the innate ability of a bacterial species to reduce the efficacy of an antibiotic through inherent structural or functional characteristic (8). P. aeruginosa is 
intrinsically resistant to most antibiotics due to the low outer membrane permeability, reported to be up to 100 times lower than that of $E$. coli (9). The outer membrane of $P$. aeruginosa, composed of a thin layer of peptidoglycan in the periplasmic space between the inner and the outer lipid membranes, reduces permeability for most antibiotics and provides an effective and adaptable armour against antimicrobial agents (10).

The bacterial cell wall membrane effectively acts as a barrier toward antimicrobial uptake, but also possess the ability to actively extrude antimicrobials that enter the cell. The resistance of $P$. aeruginosa to a wide range of antibiotics is thought to be mediated through the expression of resistance-nodulation-division (RND) efflux systems (10). Four major efflux pumps have been described for P. aeruginosa; MexAB-OprM, MexCDOprJ, MexEF-OprN and MexXY-OprM (11)(10). These pumps form a channel that spans the inner membrane, periplasm and outer membrane and facilitates the substrate to be pumped directly from the cytoplasm or cytoplasmic membrane to the extracellular environment. The combination of the low permeability of the outer membrane and the overexpression of multi-drug efflux pumps confers high intrinsic resistance to $P$. aeruginosa.

\subsubsection{Acquired resistance mechanisms}

P. aeruginosa exhibits two types of acquired resistance with horizontal gene transfer and mutational resistance. Bacterial plasmids, transposons and DNA elements can be acquired through conjugation, transformation and transduction, increasing antibiotic resistance. This mechanism is attributed to the high incidence of resistance to aminoglycosides and $\beta$-lactam antibiotics by P. aeruginosa (12)(13).

Mutational resistance describes the spontaneous mutations that lead to reduced uptake and efflux pump overexpression characteristic in resistant strains of bacteria. The 
mutation rate of bacteria increases under stressful conditions such as the presence of DNA-damaging agents. Hypermutator strains of $P$. aeruginosa exhibit a 70 -fold increase in mutation frequency and are strongly associated with infections in cystic fibrosis patients (14). Mutations involved with efflux pump expression, seen in strains with mutations in mexZ that result in the overexpression of MexXY-OprM and cause resistance to aminoglycosides, fluoroquinolones and cefepime in clinical strains of $P$. aeruginosa (15).

\subsubsection{Adaptive resistance mechanisms}

Adaptive resistance is a transient form of resistance, which is induced in the presence of antibiotics or environmental stresses and can be reversed upon removal of the external stimulant. It is induced through gene expression and protein production, or the alteration of antibiotic targets. Adaptive resistance was found in isolates sourced from P. aeruginosa infections from cystic fibrosis patients. Multiple mutations in resistance genes gyrA, gyrB and orfN were found, resulting in overproduction of efflux pumps causing resistance to ciprofloxacin.

\subsubsection{Biofilms and persister cell population}

The formation of biofilms by P. aeruginosa are a hallmark of chronic infection, indicative of disease progression and persistence. Biofilms are complex, surface-adhering microbial communities, encased within a self-composed extracellular polymeric matrix made of polysaccharides, proteins, lipids, and extracellular DNA (16)(17). The formation of biofilms involves the transition from free moving planktonic cells into a sessile, dormant state. These multicellular bacterial communities form microcolonies and develop into mature biofilms. Eventually, biofilms serve as bacterial reservoirs that are transmitted back to the environment through biofilm dispersal, leading to the colonisation of new 
surfaces and are attributed to the recalcitrance of infections (7). These bacterial communities can form on biotic or abiotic surfaces, including medical devices such as implants and catheters, as well as tissue such as the lungs of cystic fibrosis patients (18). Once established, it is challenging to penetrate or disrupt the biofilm; often resulting in surgical removal of the colonised area.

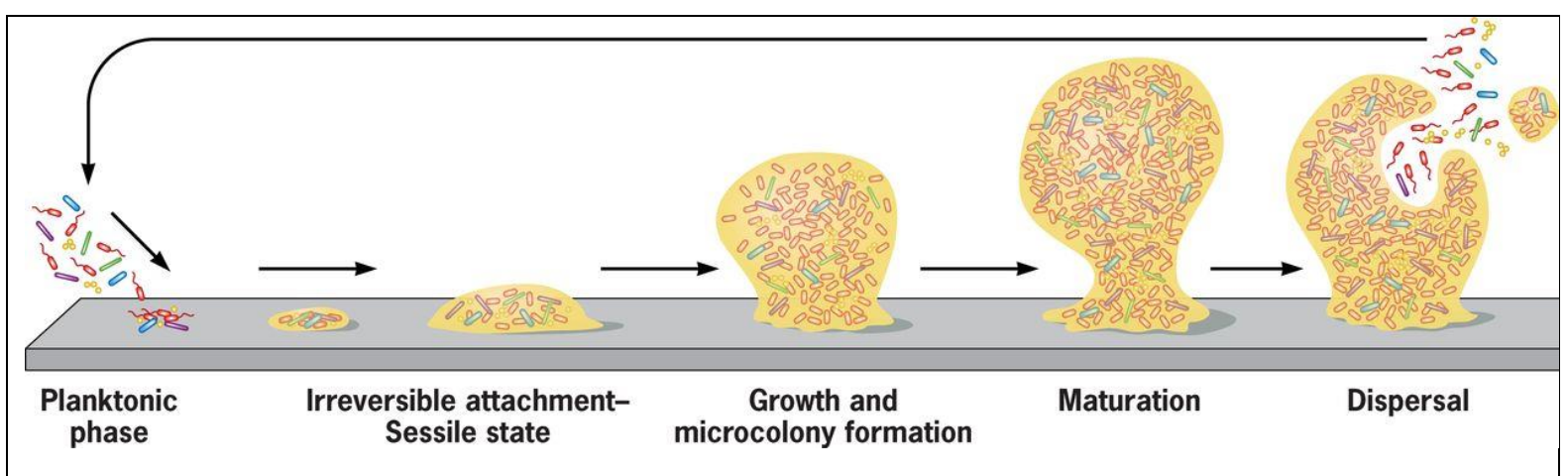

Figure 1.2 Biofilm maturation process.

Planktonic bacteria adhere to a biotic or abiotic surface, leading to irreversible attachment and transition to a sessile state of growth. These multicellular communities multiply and mature. These biofilms eventually serve as bacterial reservoirs that are transmitted back to the environment through biofilm dispersal, which facilitates the spread of infection through the colonisation of new surfaces (7). Reprinted from Clinical Microbiology Reviews, Magana et al., Options and Limitations in Clinical Investigation of Bacterial Biofilms, Apr 4, 2018, with permission by American Society for Microbiology Copyright (C) 2018

The exopolymer matrix that encases the cells of a biofilm can limit diffusion of substances, providing a protective barrier that allows effective resistance against large molecules. The negatively charged exopolysaccharide is effective in restricting permeation of positively charged aminoglycoside antibiotics, potentially through binding with them. Decreased diffusion does not restrict all agents from permeating the biofilms matrix. A decreased, often sub-inhibitory concentration of antibiotic can enter the biofilm. For $P$. aeruginosa biofilms, the combination of restricted diffusion and expression of $\beta$ lactamase act synergistically to effectively degrade the limited antimicrobials that enter the biofilm (19). 
The progression of biofilm maturation coincides with the development of biofilm-specific resistance mechanisms. These include the restricted penetration of antimicrobials into the biofilm across the exopolysaccharide matrix, the decreased growth rate of bacteria in biofilms, biofilm-specific expression of possible resistance genes, metabolic heterogeneity of the bacterial population, the overexpression of efflux systems and the presence of persister cells (20)(21)(22)(19).

It is evident the high level of recalcitrance in P. aeruginosa biofilm infections is due to an isogenic subpopulation of tolerant bacteria called persister cells or persisters (23)(16). It was reported that aggressive treatment of catheter-related bloodstream infections, with concentrations up to 1000 -fold higher than the MIC over 14 days resulted in the relapse of more than $20 \%$ of infections due to persister cells (18). These cells make up less than $1 \%$ of the bacterial population and are present in both late-stationary phase cultures and biofilm communities. These persister cells are dormant and do not multiply, giving them phenotypic tolerance rather than any genetic modification. Persister cells lie dormant during the stress of antibiotic attack, permitting the survival of the bacterial community, then can resume growth and return to planktonic cells (18). This reversible phenotypic 'switch' allows persister cells recalcitrant to antimicrobials, to emerge from the biofilm and serve as a reservoir of surviving pathogens which are responsible for recurrent infections and therapeutic failures (24).

\subsubsection{Virulence factors}

P. aeruginosa produces a variety of virulence determinants that are significant for the pathogenesis of this opportunistic nosocomial bacterium. These factors include toxins that damage the host (pyocyanin, type III secretion enzymes), factors that promote colonisation and biofilm formation (quorum sensing, adherens) and determinants that 
provide nutritional support to the bacteria (siderophores, carbohydrate permeases) $(25)(26)$.

Under iron-limiting conditions which inhibit bacterial growth, $P$. aeruginosa secretes the iron-sequestering virulence factor pyoverdine into the extracellular environment. Pyoverdine exhibits an exceptional affinity for ferric iron and can take trace iron from the environment or sequester iron from the host. Pyoverdine can scavenge iron from the host through a variety of sources, including intercepting iron from the hosts own ironsequestering proteins lactoferrin and transferrin (27) and commandeering iron from mitochondria causing significant damage (28). After the acquisition of iron, the siderophore is transferred back into the bacteria through a specific cell surface receptor, FpvA (28)(29). Pyoverdine is associated with chronic P. aeruginosa infections and has been reported as essential for P. aeruginosa pathogenesis in mammalian and invertebrate models (27).

\subsection{Current therapeutic options and emerging technologies}

With antibiotic resistance and associated systemic infections on the rise, paradoxically research and development of new antibiotics are nearly all but ceased. The high cost of development, combined with a low financial return, has led the scientific community to a crossroads; try to revitalise the current sensitivities of antibiotics or shift to the development of alternative therapeutics that employ a distinct mode of action. A combination of these plans is the current state of play.

\subsubsection{Antibiotic stewardship}

Antibiotic stewardship aims to reduce the selective pressure that promotes highly resistant pathogens by providing guidelines on the use of antibiotics. Decades of antibiotic misuse through the over-prescription by doctors and incorrect or incomplete 
antibiotic regimes by patients has contributed greatly to the current state of MDR pathogens. Antibiotic stewardship recommends the appropriate use of antibiotics and restricts the use of some classes of antibiotics, such as "last line of defence" carbapenems. It is recommended to use the highest concentration of an antibiotic, tolerated by the patient, for the shortest amount of time to eliminate the infection (4) (30). However, it is important to note there is no scientific consensus on the effectiveness of these programs to reduce the development of resistance (30).

\subsubsection{Emerging novel therapeutics}

The rise in antibiotic resistance has triggered global initiates to develop novel and more effective antimicrobial compounds as well as to develop novel delivery and targeting strategies. The development of nanostructured materials which can be used to either convey antimicrobials to assist in the delivery of novel drugs or ultimately, possess antimicrobial activity by themselves are a promising strategy (31). Additionally, nanoparticles (metallic, organic, carbon nanotube) may circumvent drug resistance mechanisms in bacteria and, associated with their antimicrobial potential, inhibit biofilm formation or other important processes.

Other strategies, including the combined use of plant-based antimicrobials and nanoparticles to overcome toxicity issues, are also being investigated. Coupling nanoparticles and natural-based antimicrobials (or other repurposed compounds) to inhibit the activity of bacterial efflux pumps; formation of biofilms and interference of quorum sensing are just some of the strategies to combat multi-drug resistant bacteria (32).

A novel strategy is the targeting of virulence factors produced by bacteria rather than the traditional antimicrobial mode of action to kill or inhibit the growth of bacteria directly. 
Kirienko and colleagues have identified small molecules that directly target the siderophore pyoverdine to reduce the pathogenic effect of pyoverdine and improved survival in C. elegans model of infection. Interestingly, these molecules reduce but do not completely inhibit pyoverdine biosynthesis (28). The targeting of quorum sensing signals in $P$. aeruginosa infections has been reported with treatment of Azithromycin, an antibiotic that inhibits lasl-mediated quorum sensing signals. Clinical trials reported improved respiratory function of CF patients compared with the placebo(33). Compromising these virulence factors does not lead to bacterial cell death; instead, it reduces the pressure to evolve resistance and could potentially be used alone or to restore and enhance antibiotic efficacy(34).

\subsection{Silver}

Silver as a therapeutic was almost forgotten after the discovery of modern-day antibiotics. However, the emergence of antibiotic resistance has led researchers to reconsider silver as a potent antimicrobial agent.

\subsubsection{History of silver as an antibacterial}

The antimicrobial properties of silver are well-established and have been utilised for centuries. The precious metal was used to make acupuncture needles during the Han Dynasty in China ca. 1500 BC, and its use as a disinfectant can be traced back to ca. 1300 BC with the preservation of liquids in clay jars lined with silver (35)(36)(37). Silver has been used in vessels, plates, and cutlery to help preserve and avoid contamination. Hippocrates is reported to have used silver in powder form to treat ulcers and infections around $400 \mathrm{BC}$ and the Romans introduced the application of silver nitrate $\left(\mathrm{AgNO}_{3}\right)$ to treat infections in $69 \mathrm{BC}(38)$. Referred to as 'lapis infernalis' translated as 'infernal stone' perhaps associated with the pain of $\mathrm{AgNO}_{3}$ application directly to the skin, has been used 
extensively as an antibacterial agent with prominent use during the 1800 s as a topical treatment of burns, ulcerations and infected wounds (39). It can be used in various forms such as solid rods or pencils to dab wounds as well as solutions varying in strengths from $0.2-2 \%(38)$.

Silver nitrate application for treatment of burn wounds was first reported in 1964, quickly followed by the development of silver sulfadiazine (AgSD) cream in 1968. AgSD cream, a combination of $\mathrm{AgNO}_{3}$ with the antibiotic sulphonamide, is a broad-spectrum silver-based antimicrobial for treatment of P. aeruginosa infections in burn wounds (39). The application of silver in a cream allows improved and prolonged delivery of silver directly to the wound compared to liquid solutions. The impressive antimicrobial activity exhibited by AgSD cream is due to the slow and sustained release of silver ions into the wound (39). Another development of this therapeutic is silvazine, a combination of AgSD $1 \%$ and chlorhexidine digluconate $0.2 \%$, with strong efficacy, reported for treatment of burns (40).

More recently, 'next generation' silver-based antimicrobials have emerged with silvercoated medical devices, like catheters, endotracheal tubes, and orthopaedic devices (41). A range of silver-coated or silver-impregnated dressings are now clinically available, which provide an effective and long-lasting concentration of silver directly to the wound site (42). Silver Nanoparticles (AgNPs) are an emerging nanotechnology with broadspectrum antibacterial activity. They can be found in a variety of household and healthcare-related products, from surface coatings of washing machines, water purifiers, wound dressings and coatings on urinary catheters and surgical instruments (43). AgNPs have shown toxic effects against a broad spectrum of Gram-negative and Gram-positive bacteria (44)(45), and displayed significant synergy with a variety of antibiotics (46). 
However, recent reports on the genotoxicity, cytotoxicity and low selectively of AgNPs have questioned the potential for AgNPs as a viable antimicrobial strategy (46).

\subsubsection{Mechanism of action}

Silver exhibits a broad-spectrum antimicrobial effect through the release of silver ions (36)(39). Silver ions have been shown to be effective against both Gram-negative and Gram-positive bacteria, as well as against some antibiotic-resistant strains after application of silver-coated dressings in an in vitro study (47). The antibacterial activity of silver has been studied extensively, resulting in some consistent trends and interesting discrepancies that preclude a definitive mode of action. It is evident that silver ions $\left(\mathrm{Ag}^{+}\right)$ disrupt multiple cellular processes leading to bactericidal action (48), while the inert form of metallic silver (Ag) does not react with human or bacterial cells.

Silver ions bind and react with proteins and enzymes on the surface of bacteria leading to critical structural changes in the cell membrane and wall, ultimately resulting in the disintegration and death of the bacteria (39)(45). Bacterial cell walls are comprised of peptidoglycan, composed of sugar and amino acid residues which are cross-linked to form a protective mesh around the cytoplasm. It is proposed that silver ions perforate the cell membrane by destroying cross-linking bonds in the peptidoglycan membrane. These pits in the membrane cause leakage of the internal cell content and contribute to cell disintegration (49)(50). Silver ions interact with structural and functional proteins such as thiol groups, leading to unstable protein folding (47). The resulting misfolded proteins on the cellular membrane contribute to an increase in permeability and a weakened structure.

Exposure to silver ions triggers the overproduction of reactive oxygen species (ROS) by disrupting metabolic pathways such as the respiratory chain in bacterial cells. Increasing 
intracellular ROS levels leads to oxidative stress, as well as protein and DNA damage. Conflicting observations have made it impossible to draw a firm conclusion from the literature on the veracity of silver ions directly inducing ROS. It seems more likely that silver ions indirectly lead to ROS production. Silver ions can perturb iron homeostasis and destabilise Fe-S clusters which are central to respiration, leading to electron leakage and associated ROS production. The affinity for silver to bind thiol groups could be hindering endogenous anti-ROS defences such as free cysteine and glutathione which are ROS scavengers (51)(50).

In summary, silver uses multiple mechanisms of action to destroy bacterial cells. Exposure to silver ions causes degradation to the bacterial cell wall, allowing the cytoplasm to leak out of the punctured membrane. Silver ions bind and penetrate the bacteria, allowing access for more silver ions to flood the bacterial cell leading to disruption of cellular and metabolic processes. Silver ions also cause genetic disruption and enzymatic inhibition halting cell growth and resulting in cell death (36). 


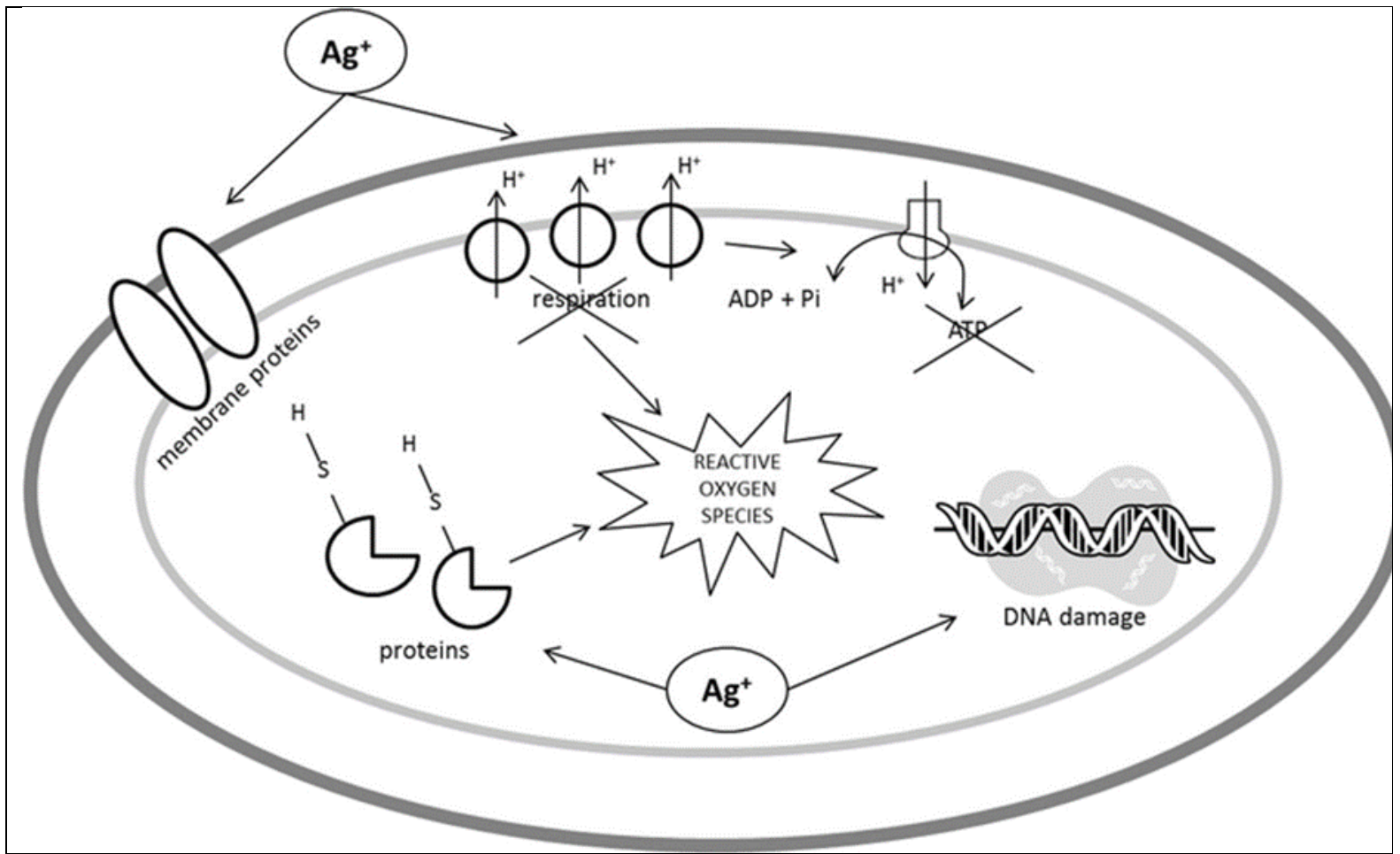

Figure 1.3 Schematic of the antibacterial mechanisms of Ag+ in bacteria.

The proposed mechanism suggests it is a multi-step process attacking DNA, cell membrane integrity and inhibition of protein and enzyme activity. Ag+ are thought to bind and form complexes with DNA, blocking replication. Disruption of cell membrane integrity by Ag+ is achieved by detaching the cytoplasmic membrane from the cell wall. Cell membrane perturbation by Ag+ is linked to the loss of proton motive force which significantly decreases cell viability. Ag+ also blocks electron transfer which is proposed to generate ROS, which are highly toxic and can damage proteins, DNA and lipids. One of the major targets of Ag+ are thiol groups ubiquitous in proteins, which could disrupt protein tertiary structure and inactivate enzymatic functions (45). Reprinted from Biometals, Kristel Mijnendonckx et al., Antimicrobial silver: uses, toxicity and potential for resistance, June 15, 2013, Copyright (C) 2013, Springer Nature

\subsubsection{Potential issues of silver use}

Silver has been used extensively for centuries as an antimicrobial treatment with only rare reports of resistance. The first silver-resistant bacteria was reported in 1960, isolated from a burn wound treated with silver nitrate (52). The best-characterised mechanism of silver resistance came from a strain of Salmonella enterica serovar Typhimurium that led to the closure of Massachusetts General Hospital burn ward and the death of several patients. The plasmid pMG101 was isolated, belonging to the IncHI incompatibility group it confers resistance to silver, mercury, tellurite as well as to 
several antibiotics (53). The historical use and present-day prevalence of silver-based therapeutics compared to the low frequency of resistance indicate the probability of future widespread silver-resistance being low.

Humans can be exposed to silver through various entry routes; with ingestion the primary route for silver compounds to enter the body. Exposure can also occur through inhalation of silver-contaminated dust, application of silver-based creams or silverimpregnated medical devices. Soluble silver compounds are easily absorbed by the body, as a result of its ability to bind to proteins, DNA and RNA. There are varying levels of recommended exposure limits and guidelines for silver, with the World Health Organisation (WHO) reporting a lifetime intake of about $10 \mathrm{~g}$ of silver for the no observable adverse effect on humans (45). Acute symptoms of chronic exposure to silver are decreased blood pressure and respiration, combined with intestinal irritation. The chronic symptoms associated with prolonged intake of low doses of silver include liver and kidney damage and changes in blood cells. A known outcome of ingestion or inhalation of silver is the development of argyria, a permanent bluish-grey discolouration of the skin or argyrosis, discolouration of the eyes that while cosmetically irreversible is not life-threatening or associated with irreversible tissue damage (54). The growing industry of silver-based products highlights the importance of understanding the potential of cytotoxicity caused by the absorption of silver in the body.

\subsection{I-Motifs}

This thesis investigates the antimicrobial activity of silver stabilised i-motifs in Gramnegative bacteria. A substantial amount of literature exists exploring the structure and potential functions of the i-motif, however, its use as an antimicrobial remains a novel 
application that holds promise for future research and development to be discussed throughout this thesis.

\subsubsection{Structure and formation of i-motifs}

DNA is polymorphic by nature, with the propensity to adopt alternative non-canonical conformations, exemplified by the structure of the i-motif. The i-motif, named for its unique intercalated-DNA structure consists of two parallel stranded duplexes intercalated in an antiparallel orientation, held together by hemi-protonated cytosine base pairs $\left(\mathrm{C}: \mathrm{C}^{+}\right)(55)$ (Figure 1.4).

Gehring et al. observed and characterised the first i-motif structure for the hexamer sequence d(TCCCCC), which formed an intercalated quadruple-helical tetramolecular structure under acidic conditions (55)(56). Examination of the i-motif structure using NMR and crystallography have indicated the i-motif can fold in either an intermolecular or intramolecular conformation. Dimers composed of two separate DNA strands or tetramers of four separate DNA strands can form an intermolecular structure or intramolecular structure (monomer) depending on the arrangement of the cytosine base pairs. The arrangement of the cytosine pairs also categories the i-motif into two different intercalation topologies known as $3^{\prime} E$ and $5^{\prime} E$; determined by the position of the terminal C:C+ base pairs at the $3^{\prime}$ or $5^{\prime}$ end (57).

Initial experiments showed that acidic conditions promote the formation of i-motif structures (58). The assumption that a low pH was necessary to ensure protonation of base pairs and confer formation of the i-motif structure led researchers to question the biological relevance of the structure; deeming the i-motif a mere structural oddity. However, recent research has provided evidence that the i-motif structure can fold under 
physiologically relevant conditions and may play a role in fundamental biological processes.

Recent studies have demonstrated the formation of i-motifs at a neutral $\mathrm{pH}$ in the presence of silver cations (55),copper cations (59) and through molecular crowding (56). The presence of metallic cations, $\mathrm{Ag}^{+}$or $\mathrm{Cu}^{+}$, act as a substrate for $\mathrm{H}^{+}$to form cytosine base pair dimers $\left(\mathrm{C}: \mathrm{C}^{+}\right)$and promote the formation of i-motif structures. This process is reversible using metal chelators or by oxidation from exposure to oxygen and presents a unique redox-sensitive control over i-motif folding. Formation of i-motif structures at neutral $\mathrm{pH}$ was also demonstrated through molecular crowding agents, such as poly(ethylene glycol) (PEG). Notably, molecular crowding mimics conditions inside the cell, supporting the in vivo existence of i-motif (56).

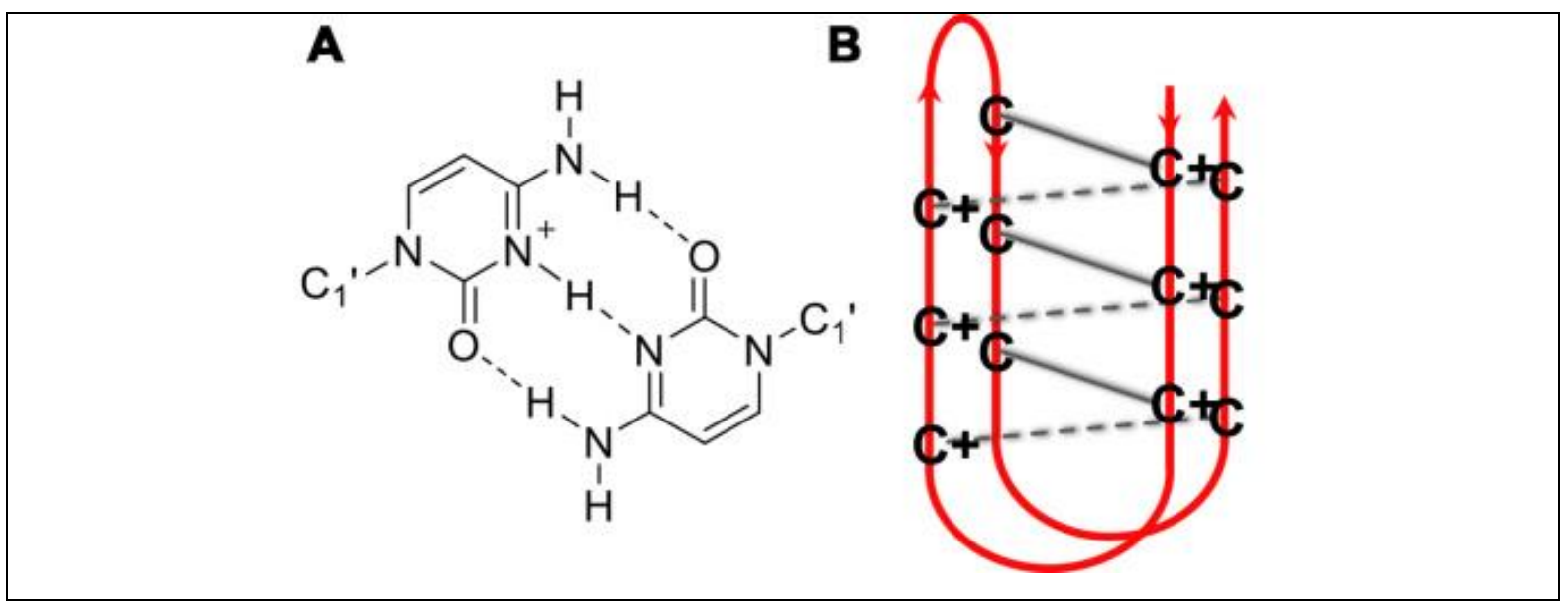

Figure 1.4 Predicted structure of i-motif.

(a) the hemi-protonated C-C+ base pair (b) i-motif structure consisting of two parallel stranded duplexes intercalated in an antiparallel orientation, held together by hemi-protonated cytosine base pairs. Figure reprinted from (Abdelhamid et al., 2018) with permission from Oxford University Press, Copyright (C) 2018 (57) 


\subsubsection{Biological relevance}

There is growing evidence that i-motifs play a fundamental role in gene regulation. Imotif structures form in cytosine-rich sequences complementary to guanine-rich Gquadruplex structures in the gene promoter regions of the human genome. Zeerati et al., first detected i-motif structures in the nuclei of human cells using antibody fragments (iMab) that recognise i-motif structures with high affinity and selectivity (56). This study also explored the connection between i-motif formation and the cell cycle; finding imotifs are predominant during the late G1 phase of the cell, which is linked to high levels of transcription.

Several studies have explored the regulatory role of i-motifs in the modulation of transcription and replication. Sun et al. investigated transcriptional regulation in the cMyc promotor; using a supercoiled plasmid to mimic the negative superhelicity of transcription they were able to induce the formation of i-motif and G-quadruplex structures under physiological conditions suggesting both non-canonical DNA structures are involved in the control of gene expression (60). The role of i-motif structures during DNA replication was linked to the progression of disease. It was discovered that i-motifs form in the presence of molecular crowding and could inhibit DNA polymerase stalling DNA replication, proposing the i-motif could play a role in the initiation of genomic instability through inhibition of DNA replication which in turn, could lead to diseases such as cancer (61). The presence of i-motif structures in the regulatory regions of the human genome, such as telomeric and promotor regions confer a critical regulatory role in the genome; with dis-regulation linked to disease. Overexpression of transcription in oncogenes such as c-Myc, BCL2 and K-Ras are seen in cancer cells, with underexpression commonly linked to neurodegenerative diseases (62). 
Together the current literature on i-motifs provides insight into the unique structure and stability of the structure, as well as its potential role in gene regulation and expression with implications for disease

\subsubsection{Current applications of the i-motif}

Since their discovery, i-motifs have been applied to nanotechnology for analytical and biomedical purposes. The i-motifs ability to rapidly and reversibly form a unique intercalated tetraplex structure, sensitive to changes in $\mathrm{pH}$ has made it an attractive tool to researchers. Several nanodevices have been created based on the structural oscillation between folded and unfolded to monitor $\mathrm{pH}$ changes inside cells. The development of the 'I-switch' by the Krishnan group was the first example of an i-motif-based nanotechnology able to sense and report pH changes along endosomal maturation both inside living cells in culture and in a multicellular organism (63). The use of gold nanoparticles (AuNPs) modified to i-motif forming sequences have been used to effectively monitor $\mathrm{pH}$ changes inside endocytic vesicles (64).

The substantial evidence that the i-motif structures form in gene promoter regions and play a fundamental role in gene regulation present the i-motif as an attractive drug target with several studies show promise of targeting i-motifs sequences for chemotherapy and modulation of gene transcription. A recent study demonstrated the anti-cancer drug mitoxantrone can bind and induce stable i-motif structures at a physiologically relevant $\mathrm{pH}(65)$.

\subsection{Research aims}

The overall aim of this thesis was to investigate the antibacterial activity of silver i-motifs against Gram-negative $P$. aeruginosa, contributing to the research against resistant bacteria. The increase of MDR strains of bacteria, exemplified by the ESKAPE pathogens 
is a global health concern. The development of new antibiotics has slowed in combination with a dwindling antibiotic armamentarium, leading researchers to explore novel antimicrobial options. Investigation of the antibacterial activity of AgiMs was explored in three stages.

Silver stabilised i-motifs are well reported in the literature but are currently not established as an antibacterial agent. The first aim was to optimise the AgiMs preparation protocol and explore the effect of different poly-C sequences lengths to achieve the optimal antibacterial activity. The objective was to select a candidate AgiM sequence to use throughout the study, which was most efficacious per nucleotide and could provide a potentially viable treatment with high efficacy and reduced toxicity.

The second aim was to assess the in vitro antibacterial activity of AgiMs. The ability of AgiMs to deliver soluble $\mathrm{Ag}^{+}$to $P$. aeruginosa and $A$. baumannii was assessed using a variety of susceptibility tests, indicating a strong antibacterial activity. This led to the final aim of this thesis, to validate AgiMs antibacterial activity using an in vivo invertebrate model to elucidate the potential implications this treatment could have in a clinical setting. 



\section{CHAPTER 2. MATERIALS AND METHODS}

\subsection{Bacterial Strains}

Bacterial strains were stored at $-80{ }^{\circ} \mathrm{C}$ in Lysogeny broth (LB) supplemented with $25 \%$ glycerol.

Table 2-1 Bacterial strains used in the study.

* New Zealand Culture Collection (NZCC), Environmental Science and Research, ${ }^{* *}$ provided by Dr Matt Blakiston at Auckland City Hospital, originally sourced from patients in NZ and the Pacific

\begin{tabular}{|c|c|c|}
\hline Strain & Characteristics & Source \\
\hline \multicolumn{3}{|c|}{$\begin{array}{l}\text { Pseudomonas } \\
\text { aeruginosa }\end{array}$} \\
\hline PA01 & ATCC 15692: Laboratory strain & NZCC* \\
\hline ARL 1079 & Clinical sample: tracheal aspirate & NZCC* \\
\hline ARL 1205 & Clinical sample: left thigh & NZCC* \\
\hline ARL 1024 & Clinical sample: catheter, urine & NZCC* \\
\hline PA- $\Delta$ mexB & $\begin{array}{l}\text { PA01 two-allele transposon mutant: } \\
\text { Resistance-nodulation-cell division } \\
\text { multidrug efflux transported MexB }\end{array}$ & $\begin{array}{l}\text { University of } \\
\text { Washington } \\
\text { (Manpoil Lab) }\end{array}$ \\
\hline \multicolumn{3}{|c|}{$\begin{array}{l}\text { Acinetobacter } \\
\text { baumannii }\end{array}$} \\
\hline$A B$ & Laboratory strain & Auckland Hospital** \\
\hline$A B 6$ & Clinical sample & Auckland Hospital** \\
\hline$A B 10$ & Clinical sample & Auckland Hospital** \\
\hline$A B 12$ & Clinical sample & Auckland Hospital** \\
\hline
\end{tabular}

\subsection{Oligonucleotides}

Oligonucleotides used in this study were synthesised by Integrated DNA Technologies (IDT, Singapore). The lyophilised oligonucleotides were re-suspended in TE buffer (10 mM Tris-HCl, $1 \mathrm{mM}$ EDTA, $\mathrm{pH} 8.0$ ) to a concentration of $250 \mu \mathrm{M}$, and stored at $-20^{\circ} \mathrm{C}$. Sequences used in this study are given in Table 2-2.

Table 2-2 Oligonucleotide sequences used in this study.

Underlined sequences denote the i-motif turn 


\begin{tabular}{|c|c|c|}
\hline Sequence ID & Sequence $\left(5^{\prime}-3^{\prime}\right)$ & Number of $\mathrm{C}-\mathrm{C}^{+}$pairs \\
\hline Half i-motif 1 & TCCC $\underline{\text { TAA CCCT }}$ & 3 \\
\hline Half i-motif 2 & 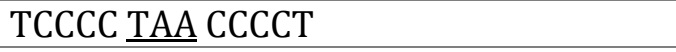 & 4 \\
\hline Half i-motif 3 & TCСССС TAA СССССТ & 5 \\
\hline Full i-motif 1 & 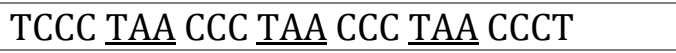 & 6 \\
\hline Full i-motif 2 & 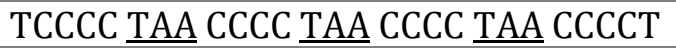 & 8 \\
\hline Full i-motif 3 & $\begin{array}{l}\text { TCСССС TAA CСССС TAA CСССС TAA } \\
\text { CССССТ }\end{array}$ & 10 \\
\hline
\end{tabular}

\subsection{Reagents and Chemicals}

\subsubsection{Antibiotics}

Antibiotic stocks were prepared in $\mathrm{ddH}_{2} \mathrm{O}$ and stored at $-20^{\circ} \mathrm{C}$. Antibiotics used in this study were purchased from Sigma-Aldrich NZ and ThermoFisher Scientific NZ (Table 2$3)$.

Table 2-3 Antibiotic agents used in this thesis

\begin{tabular}{ll}
\hline Antibiotic Stock solutions & Stock concentration \\
\hline Tobramycin & $1 \mathrm{mg} / \mathrm{mL}$ \\
\hline Ciprofloxacin & $1 \mathrm{mg} / \mathrm{mL}$ \\
\hline Antibiotic Antimicrobial Susceptibility Discs & $10 \mathrm{mcg}$ \\
\hline Oxoid Tobramycin & $10 \mathrm{mcg}$ \\
\hline Oxoid Ciprofloxacin & $100 \mathrm{mcg}$ \\
\hline Oxoid Piperacillin & $30 \mathrm{mcg}$ \\
\hline Oxoid Cefotaxime & $10 \mathrm{mcg}$ \\
\hline Oxoid Gentamicin & $30 \mathrm{mcg}$ \\
\hline Oxoid Tetracycline & \\
\hline
\end{tabular}

\subsubsection{Chemicals}

All chemicals and reagents used in this study were obtained from Sigma-Aldrich or

ThermoFisher Scientific NZ. Buffers and solutions used are described in Appendix 1.1 


\subsection{Growth media and agar}

\subsubsection{Lysogeny broth (LB)}

The standard growth culture medium used for experiments was lysogeny broth, Miller variant (LB). LB media was prepared by mixing $10 \mathrm{~g}$ Tryptone (Oxoid LP0042), 5 g Yeast extract (Oxoid LP0021) and $10 \mathrm{~g} \mathrm{NaCl}$ with $\mathrm{ddH}_{2} \mathrm{O}$ to a volume of $1 \mathrm{~L}$. The $\mathrm{pH}$ was adjusted to 7.0 , and the solution sterilised by autoclave at $121^{\circ} \mathrm{C}$ for $20 \mathrm{~min}$. The sterile media was stored at room temperature. A double-strength media (2xLB) was prepared with double the quantity of Typtone, Yeast and $\mathrm{NaCl}$ in $1 \mathrm{~L}$ of $\mathrm{ddH}_{2} \mathrm{O}$.

\subsubsection{LB agar}

LB media was prepared as above, with the addition of $15 \mathrm{~g}$ bacteriological agar (Oxoid LP0011) per litre of media prior to sterilisation by autoclave. The sterile agar was cooled to $50^{\circ} \mathrm{C}$ in a water bath then aseptically poured into $90 \mathrm{~mm}$ Petri dishes ( $25 \mathrm{~mL}$ per plate) and left to solidify at room temperature before storage at $4{ }^{\circ} \mathrm{C}$.

\subsubsection{Mueller Hinton broth}

Mueller Hinton broth was used in Kirby-Bauer antibiotic susceptibility testing. Mueller Hinton media was prepared by mixing $21 \mathrm{~g}$ Mueller Hinton Broth (Oxoid CM405) with $\mathrm{ddH}_{2} \mathrm{O}$ to a volume of $1 \mathrm{~L}$ and sterilising by autoclave at $121{ }^{\circ} \mathrm{C}$ for $20 \mathrm{~min}$.

\subsubsection{Mueller Hinton agar}

Mueller Hinton agar was prepared by suspending $38 \mathrm{~g}$ of Mueller Hinton agar base (Oxoid CM0337) in $1 \mathrm{~L}$ of $\mathrm{ddH}_{2} \mathrm{O}$ and boiling until medium dissolved completely. The solution was then sterilised by autoclave at $121{ }^{\circ} \mathrm{C}$ for $20 \mathrm{~min}$. The sterile agar was cooled to 50 ${ }^{\circ} \mathrm{C}$ in a water bath then aseptically poured into $90 \mathrm{~mm}$ Petri dishes (25 $\mathrm{mL}$ per plate) and left to solidify at room temperature before storage at $4{ }^{\circ} \mathrm{C}$. 


\subsubsection{Pseudomonas selection agar}

To prepare Pseudomonas selection agar, 24.2 g of Pseudomonas agar base (Oxoid, CM0559) was re-suspended in $500 \mathrm{~mL}$ of $\mathrm{ddH}_{2} \mathrm{O}$, and $5 \mathrm{~mL}$ of glycerol, then boiled until dissolved completely. The solution was sterilised by autoclaving at $121^{\circ} \mathrm{C}$ for $20 \mathrm{~min}$ then cooled to $50{ }^{\circ} \mathrm{C}$ in a water bath. The contents of one vial of Pseudomonas C-F-C supplement (Oxoid SR102), reconstituted in 1:1 Ethanol: $\mathrm{dd}_{2} \mathrm{O}$, was added, mixed well, then the media poured into $90 \mathrm{~mm}$ Petri dishes aseptically (25 $\mathrm{mL}$ per plate) and left to solidify at room temperature before storage at $4{ }^{\circ} \mathrm{C}$.

\subsection{Silver stabilised i-motifs synthesis}

AgiM were prepared by rapidly mixing $250 \mu \mathrm{M}$ oligonucleotide and $1 \mathrm{mM} \mathrm{AgNO}_{3}$ in 100 mM MES buffer (pH 6.5) to a final volume of $400 \mu \mathrm{L}$. The resulting solution was incubated in the dark at $4^{\circ} \mathrm{C}$ for 30 mins. Excess $\mathrm{Ag}^{+}$and reagents were removed using Amicon Ultra-0.5 mL centrifugal filters Ultralcel - 3K (Merck Millipore., Germany), and resulting filtrate re-suspended in $100 \mu \mathrm{L}$ milliQ $\mathrm{H}_{2} \mathrm{O}$ (Millipore co., USA). The ratio of DNA : $\mathrm{Ag}^{+}$was altered during preparation of AgiM depending on molar excess of $\mathrm{Ag}^{+}$required. For 1:6 DNA : $\mathrm{Ag}^{+}\left(20 \mu \mathrm{L}\right.$ Oligonucleotide: $\left.30 \mu \mathrm{L} \mathrm{AgNO}_{3}\right)$ to $1: 10 \mathrm{DNA}: \mathrm{Ag}^{+}(20 \mu \mathrm{L}$ Oligonucleotide: $\left.50 \mu \mathrm{L} \mathrm{AgNO}_{3}\right)$ and 1:20 DNA : $\mathrm{Ag}^{+}(20 \mu \mathrm{L} \text { Oligonucleotide: } 100 \mu \mathrm{L} \mathrm{AgNO})_{3}$.

\subsection{Determination of Antimicrobial activity}

\subsubsection{Bacterial growth conditions}

Frozen glycerol stocks of bacteria were subcultured onto agar plates, incubated overnight at $37^{\circ} \mathrm{C}$ then kept inverted at $4{ }^{\circ} \mathrm{C}$ over 30 days, to be used as stock bacterial agar plates. Bacterial cultures were grown in LB medium, prepared from liquid cultures or bacterial agar plates, and incubated at $37{ }^{\circ} \mathrm{C}$ with $200 \mathrm{rpm}$ shaking with aeration for $16-\mathrm{h}$. Overnight cultures were diluted 1:100 into fresh LB media and grown at $37^{\circ} \mathrm{C}$ until an 
$\mathrm{OD}_{600}$ of 0.2 - 0.4 was obtained, to ensure cells had reached the exponential phase prior to use.

\subsubsection{Standard growth assay protocol}

Standard growth assays were performed in 96-well micro-titre plates using a SPECTROstar Nano absorbance plate reader (BMG Labtech, Germany) to measure bacterial growth by optical density at $600 \mathrm{~nm}\left(\mathrm{OD}_{600}\right)$. Wells containing test substrate (AgiM) at a volume of $75 \mu \mathrm{L}$ were inoculated with $10^{5} \times \mathrm{CFU} / 75 \mu \mathrm{L}$ exponentially growing bacteria, to a total well volume of $150 \mu \mathrm{L}$. Control wells containing bacteria-only, and blank wells with LB-only were used as controls for bacterial growth and contamination, respectively. Plates were then incubated at $37^{\circ} \mathrm{C}$ with aeration by meander-corner-well shaking and 10 seconds of $200 \mathrm{rpm}$ orbital shaking before each reading and growth measured every 5 mins for 20 -h. Results were blank corrected; then the $\mathrm{OD}_{600}$ plotted against time to provide growth curves for the various treatments.

\subsubsection{Optical density by UV spectroscopy}

The optical density (OD) of bacterial cultures was measured using the Unicam Helios spectrophotometer (Biolab Scientific Ltd, CA) at 600nm. Samples were measured in $1 \mathrm{~mL}$ cuvettes, with LB media as the blank control.

\subsubsection{MIC determination}

The minimum inhibitory concentration (MIC) was determined using the microdilution method as described by CLSI. A standard growth assay was prepared (Methods 1.2.2.2), with serial two-fold dilutions of test substrate in sterile milliQ $\mathrm{H}_{2} \mathrm{O}$, obtaining a concentration range of $2 \mu \mathrm{M}$ to $0.0075 \mu \mathrm{M}$. Then, $1 \times 10^{5} \mathrm{CFU} / 75 \mu \mathrm{L}$ bacterial suspension were added to the wells containing the diluted test substrate. After incubation for 20 -h at 
$37{ }^{\circ} \mathrm{C}$ wells were observed, and MIC recorded as the lowest concentration of test substrate that inhibited bacterial growth determined by absence of visual turbidity.

\subsubsection{Pyoverdine assay}

Pyoverdine assays were prepared according to standard growth assay protocol (see Methods 1.2.2.2), except an Enspire 2300 Multilabel plate reader (Perkin Elmer, USA) was used. To assess pyoverdine production during growth, wells were excited at $405 \mathrm{~nm}$, and corresponding emission wavelength at $455 \mathrm{~nm}$ was recorded every 5 mins for 20-h.

\subsubsection{Antibiotic susceptibility testing}

Antibiotic susceptibility tests of the bacterial strains were performed by Kirby-Bauer Disc diffusion test. The test strains were cultured in both LB media and Mueller Hinton media at $37^{\circ}$ overnight, then diluted to $1 \times 10^{8} \mathrm{CFU} / \mathrm{mL}$ (comparable to $0.5 \mathrm{McF}$ arland Standard) and uniformly swabbed onto the surface of LB agar and Muller Hinton agar plates respectively. After 5 minutes of agar plates being allowed to stand, antibiotic discs recommended by the Clinical Laboratory Standards Institute (CLSI) were placed onto agar plates using sterile forceps then incubated at $37^{\circ}$ for $16-18$ hours. Antibiotics discs tested were Tobramycin, Ciprofloxacin, Piperacillin, Cefotaxime, Tetracycline, and Gentamicin. Following incubation, agar plates were covered in a bacterial lawn with zones of inhibition around the antibiotic discs. Bacterial susceptibility to these antibiotics was verified by measuring the diameter (or radius and multiplying by 2) of the inhibition zones formed over the test period.

\subsection{Biofilm eradication testing}

\subsubsection{Biofilm assay}

Biofilm assays were performed using MBEC peg plates (Innovotech, CA). Exponentially growing bacterial strains suspended in LB were adjusted to $1 \times 10^{5} \mathrm{CFU} / \mathrm{mL}$ and used to 
inoculate the 96-well plate with a well volume of $150 \mu \mathrm{L}$. The MBEC peg lid was placed into the wells and incubated in a humidified container at $37^{\circ} \mathrm{C}$ with $110 \mathrm{rpm}$ shaking for 20 or 44 hours to allow biofilm formation.

After biofilm growth for 20 or 44 hours, the peg lid was removed and washed in $200 \mu \mathrm{L}$ sterile $\mathrm{ddH}_{2} \mathrm{O}$ then placed into a new 96 -well plate containing the antimicrobial challenge and incubated for 6 -h in a humidified container at $37^{\circ} \mathrm{C}$ with $110 \mathrm{rpm}$ shaking. Challenge plates contained $200 \mu \mathrm{L}$ of AgiM at 2-fold dilutions from $5 \mu \mathrm{M}$ to $0.6 \mu \mathrm{M}$. After challenge, biofilm growth and eradication was analysed by various methods.

\subsubsection{Inhibition of planktonic growth}

After the antimicrobial challenge, the ability of the treatment to inhibit planktonic growth was investigated by measuring the $\mathrm{OD}_{600}$ of the challenge plate after removal of the peg lid.

\subsubsection{Biofilm biomass determination}

The biomass of biofilms formed on the peg lid was determined by crystal violet staining. After the antimicrobial challenge, the peg plate was removed and stained in $200 \mu \mathrm{L} 0.05 \%$

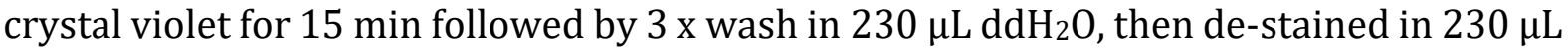
$100 \%$ EtOH overnight followed by the measurement of the OD595 value.

\subsection{Time-kill assay}

Time-kill curve analyses were performed using a micro-dilution method in a 96-well micro-titration plate. A starting inoculum of $2 \times 10^{5} \mathrm{CFU}$ exponential bacteria was incubated in the presence of AgiM concentrations set $1 \times$ MIC and $4 \times$ MIC, at room temperature for 6-h. MICs for each bacterial strain were determined prior using a standard growth assay protocol. At time points of 15 mins, 30 mins, 1-h, 2-h, 3-h, 4-h and 6-h, the contents of corresponding wells $(150 \mu \mathrm{L})$ were removed and serially diluted to 
enumerate viable cells (CFU). The CFU counts for each time point were compared to the untreated control CFU count.

\subsection{Viable cell counts}

Bacterial cell viability was measured by enumeration of colony-forming units on agar plates. Growing bacteria were removed from 96 -well plates at specific time points and diluted in sterile 1 x PBS in 1:10 dilutions (20 $\mu \mathrm{L}$ culture in $180 \mu \mathrm{L}$ diluent). Droplets of $10 \mathrm{uL}$ of each dilution were spotted on LB agar or Mueller Hinton agar. Once fully absorbed, plates were inverted and incubated at $37^{\circ} \mathrm{C}$ for 20 -h. CFU enumeration was determined by counting the colonies for the first dilution that resulted in a countable range of 10- 100 colonies and $\mathrm{CFU} / \mathrm{mL}$ calculated.

\subsection{Checkerboard synergy testing}

Investigation of synergistic interactions between AgiM and tobramycin was achieved using the checkerboard microdilution method (Ref). The MIC for test agents was determined prior to checkboard assays using a standard growth assay protocol described in methods 1.2.2.4.

Synergy checkerboard assays were performed in 96-well micro-titre plates. Test agents, tobramycin and AgiM, were prepared to $4 \mathrm{x}$ concentration using milliQ $\mathrm{H}_{2} \mathrm{O}$ to account for in-plate dilution. Tobramycin was added along the y-axis of the plate, using a 2-fold serial dilution to achieve a concentration range from high to low. AgiM was added along the $\mathrm{x}$ axis of the plate, using a 2-fold serial dilution to achieve a concentration range from high to low, as illustrated in Figure 1. Then wells were inoculated with $2 \times 10^{5} \mathrm{CFU}$ of exponential bacteria suspended in LB. Control wells containing LB media or bacteria only were included in all experiments. Bacterial growth was measured using a SPECTROstar 
plate at $37^{\circ} \mathrm{C}$ with aeration by meander-corner-well shaking and 10 seconds of $200 \mathrm{rpm}$ orbital shaking before each reading. The $\mathrm{OD}_{600}$ was determined at 5-minute intervals over 20-h.

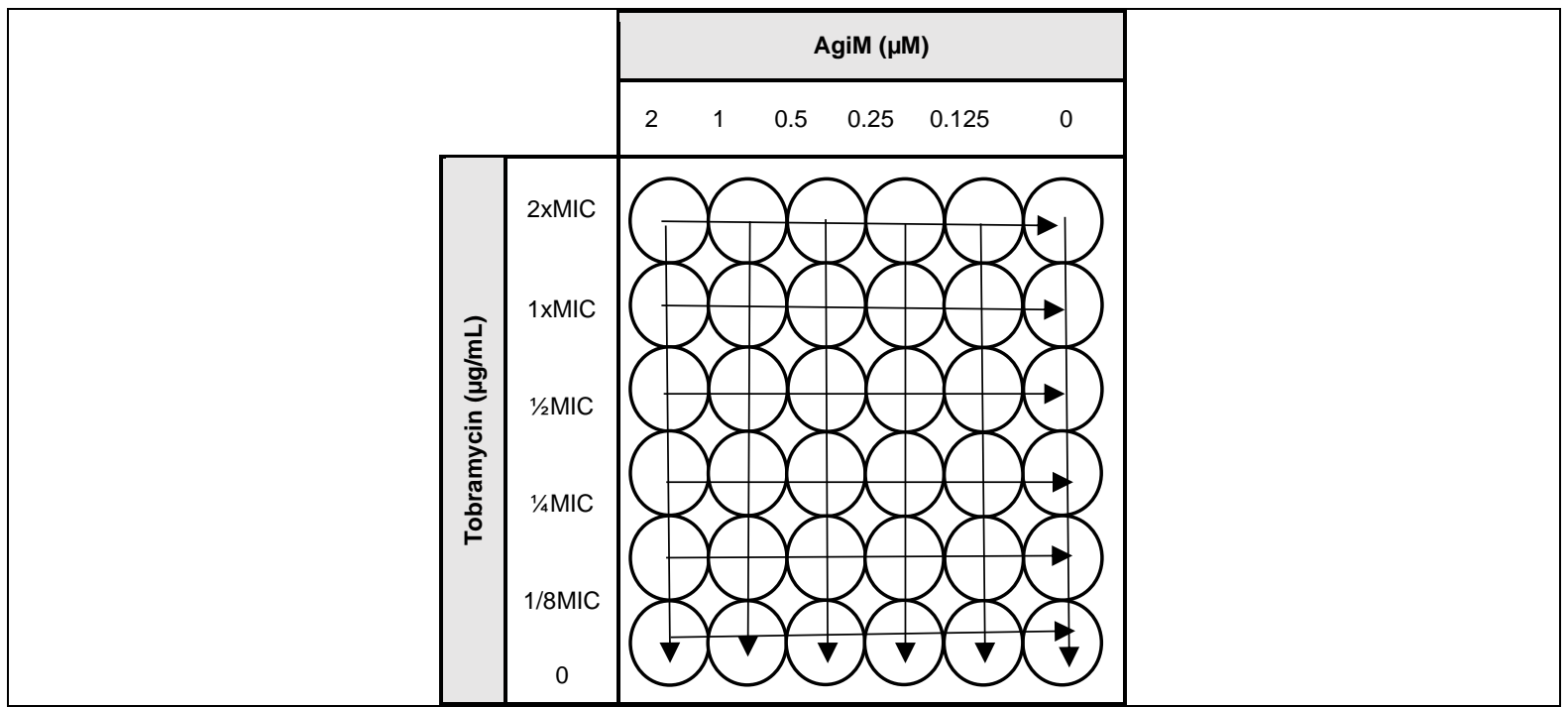

Figure 2.1 Schematic of checkerboard synergy plate layout in 96-well micro-titre plate.

Tobramycin was added along the y-axis of the plate, using a 2-fold serial dilution to achieve a concentration range from high to low. AgiM was added along the x-axis of the plate, using a 2-fold serial dilution to achieve a concentration range from high to low

\title{
2.10.1. FIC determination for synergy testing
}

To evaluate the interactions of the two test agents at the completion of the checkerboard assay, the fractional inhibitory concentration (FIC) index was calculated.

At the completion of checkerboard assay $\mathrm{MIC}_{\text {combination }}$ and $\mathrm{MIC}_{\text {individual }}$ values were determined for agent A (tobramycin) and agent B (AgiM). Synergy was determined by calculation of fractional inhibitory concentration (FIC) using the equation:

\author{
$\Sigma$ FIC $=$ FIC agent A + FIC agent B \\ FIC agent $\mathrm{A}=\mathrm{MIC}_{\mathrm{A} \text { combination }} \div \mathrm{MIC}_{\mathrm{A} \text { alone }}$ \\ FIC agent $\mathrm{B}=\mathrm{MIC}_{\mathrm{B} \text { combination }} \div \mathrm{MIC} \mathrm{C}_{\mathrm{B}}$ alone
}


Resulting $\sum$ FIC values were interpreted to be synergistic (FIC $\left.\leq 0.5\right)$, partial synergy $(0.5$ $<$ FIC $<1.0$ ), indifferent $(1.0 \leq$ FIC $\leq 4.0)$ or antagonistic (FIC $>4.0$ ).

Table 2-4 FIC interpretations for evaluation of synergistic activity

FIC RESULT SYNERGY

PARTIAL SYNERGY

INDIFFERENT

ANTAGONISM
INTERPRETATIONS

FIC $\leq 0.5$

$0.5<$ FIC $<1.0$

$1.0 \leq$ FIC $\leq 4.0$

FIC $>4.0$

\subsection{In vivo antimicrobial testing}

\subsubsection{Galleria mellonella infection}

The greater wax moth (Galleria mellonella) were used as the in vivo model of infection during antimicrobial testing. Last instar larvae were purchased from Biosuppliers (NZ), specified to be antibiotic-free and were stored in the dark at room temperature for up to 14 days. Larvae between 150-210 mg in weight were selected for experiments and starved overnight at $37^{\circ}$ degrees. Prior to injection, larvae were anaesthetised by placing into petri dishes (Interlab, NZ) on ice for 15 minutes. Larvae were injected with $10 \mu \mathrm{L}$ of the prepared bacterial suspension through the right hind proleg into the circulating haemolymph. To control the injection volume, an automated syringe pump (KD Scientific, United States) was used (speed to $1 \mu \mathrm{L} / \mathrm{sec}$ ) with a $1 \mathrm{~mL}$ syringe. Larvae were injected with $10 \mu \mathrm{L}$ of the prepared bacterial suspension through the right hind proleg into the circulating haemolymph. After 30 minutes, treatments were administered as a second injection of $10 \mu \mathrm{L}$ into the left hind proleg at the stated concentration. Larvae injected with PBS only, and treatment only were used as controls for injection-related mortality and treatment toxicity, respectively. After injection, larvae were kept starved in plastic 
petri dishes at $37^{\circ} \mathrm{C}$ and monitored for mortality every hour from $12-24 \mathrm{~h}$ post-injection. Death was characterised by melanisation of the larval skin resulting in the larvae turning dark brown to black, and unresponsive to touch. The death counts from each time point were used to generate Kaplan-Meier survival curves. Bacteria was plated on an agar plate to determine CFU and confirm the approximate number of live bacteria used to infect each larvae.

\subsubsection{Determination of bacterial load in the haemolymph}

Infected larvae were collected, and the larval skin was disinfected by sterile swab of ethanol. The larvae were anaesthetised on ice for 15 minutes, or until no movement was detected. The haemolymph was collected from each larva into individual Eppendorf tubes by holding the larva with tweezers and making an incision between the two segments near the tail of the larvae using a sterilised stainless-steel scalpel No.10 (Swann-Morton, England). A sample of $10 \mu \mathrm{L}$ haemolymph was extracted from the incision site with a pipette and added to $1 \mathrm{~mL} 1$ x PBS. The resulting solution was immediately serially diluted at a 1: 10 ratio (20 $\mu \mathrm{L}$ into $180 \mu \mathrm{L}$ PBS) and $10 \mu \mathrm{L}$ aliquots plated onto Pseudomonas selection agar plates in triplicates. Plates were allowed to dry, then inverted and incubated at $37{ }^{\circ} \mathrm{C}$ overnight. This process was repeated for each of the larvae sampled per treatment group. Colonies were counted the next day, and CFU/mL haemolymph was calculated.

\subsection{Graphing and Statistical analysis}

GraphPad Prism 6 (GraphPad Software, USA) was used for all statistical analysis and graphing. Analysis of variance was used to determine statistical significance in data. Oneway ANOVAs determined significance between the means of one variable for two or more independent groups and Two-way ANOVAs were used to determine statistically 
significant differences in means between groups with two independent variables. Dunnett's post-hoc test was used to compare group means to the control mean and Tukey's post-hoc test compared the means between all groups. A p-value of less than 0.05 was considered significant, with significance indicated as ${ }^{*} \mathrm{p}<0.05,{ }^{* *} \mathrm{p}<0.01,{ }^{* * *} \mathrm{p}<0.001$. 


\section{CHAPTER 3. OPTIMISATION OF AGIM FOR ANTIBACTERIAL}

\section{ACTIVITY}

\subsection{Aims and rationale}

The i-motif is a unique non-canonical DNA structure, consisting of two parallel stranded duplexes intercalated in an antiparallel orientation, held together by hemi-protonated cytosine base pairs (C: $\left.\mathrm{C}^{+}\right)(57)$. Day and colleagues first reported that the presence of $\mathrm{Ag}^{+}$ promotes the stable folding of DNA into the i-motif conformation at room temperature and a physiological $\mathrm{pH}$ (66). The formation of i-motifs in poly-c sequences with the addition of $\mathrm{Ag}^{+}$is also a known intermediate prior to silver nanocluster (AgNC) reduction (67). Previous experiments in our Laboratory investigating the antibacterial activity of AgNCs found that the intermediate conformation of AgiMs exhibited enhanced antibacterial killing compared to the equivalent AgNCs. The antibacterial activity was proposed to be due to the available $\mathrm{Ag}^{+}$in the AgiMs compared to the $\mathrm{A}^{0}$ in AgNCs. This chapter follows on from that preliminary research, with the aim of optimising the protocol of silver stabilised i-motifs (AgiMs) to achieve antibacterial potency against $P$. aeruginosa.

Oligonucleotide sequences were designed for this study with identical connecting loops (TAA) and varying poly-C tracts. These sequences included three full i-motifs and the equivalent three half i-motifs, which are predicted to form intra-strand i-motifs and interstrand dimeric i-motifs respectively. The preparation of AgiMs for this thesis was modified from Javani and colleagues protocol for AgNCs (68) The protocol instructs a 6:1 Ag+: DNA molar ratio recommended to limit the formation of silver nanoparticles (AgNPs) 
created by the reduction of excess $\mathrm{Ag}^{+}$during the synthesis of AgNCs. However, the reduction step is not required during the formation of AgiMs, as the addition of $\mathrm{Ag}^{+}$ induces the rapid and stable formation of i-motifs at room temperature followed by filtration to ensure removal of all unbound $\mathrm{Ag}^{+}$; allowing higher molar ratios to be explored in this study.

Preliminary experiments indicated full i-motifs and half i-motifs exhibited similar antibacterial activity, which is not what was expected. It was proposed that full i-motifs capable of forming intra-strand conformations would be twice as effective (on a molar basis) as their half i-motif counterparts which form inter-strand dimeric conformations. It was hypothesised that the recommended 6:1 $\mathrm{Ag}^{+}$: DNA molar ratio was not sufficiently saturating the larger full $\mathrm{i}$-motif sequences with $\mathrm{Ag}^{+}$, limiting the amount of bound silver and corresponding antibacterial activity. An experiment was designed to test this hypothesis using a standard growth assay to compare the antimicrobial activity of the 6:1 $\mathrm{Ag}^{+}$: DNA molar ratio with an excess of 20:1 $\mathrm{Ag}^{+}$: DNA molar ratio in full i-motif structures and 10:1 $\mathrm{Ag}^{+}$: DNA in half i-motif structures in the laboratory strain of P. aeruginosa PA01. The correlation between the poly-C tract of the i-motif sequence and antibacterial activity was investigated. It is reported that $\mathrm{Ag}^{+}$bind at the $\mathrm{N} 3$ atom of cytosine in $\mathrm{C}^{-} \mathrm{C}^{+}$base pair duplexes with hydrogen bonds aiding in stabilisation of the complex (69). Because of this binding mechanism is was proposed that each $\mathrm{C}_{-} \mathrm{C}^{+}$pair is capable of binding one $\mathrm{Ag}^{+}$, indicating a direct correlation to poly-C length and antibacterial activity. An experiment was designed to investigate this hypothesis using a standard growth assay to compare the antibacterial activity of the i-motif sequences. 


\subsection{Results}

\subsubsection{AgiMs exhibit antibacterial activity}

To investigate the optimal $\mathrm{Ag}^{+}$: DNA molar ratio for antibacterial activity, a full intrastrand i-motif (full i-motif 2: TCCCCTAACCCCTAACCCCTAАCCCT) and corresponding half inter-strand dimeric i-motif (half i-motif 2: TCCCCTAАССССТ) were selected. It was expected that in the presence of $\mathrm{Ag}^{+}$, the full $\mathrm{i}$-motif sequences would form twice as many i-motif structures resulting in an approximately 2-fold increase in efficacy compared to the half i-motif sequences.

The i-motif sequences were prepared using the 6:1 $\mathrm{Ag}^{+}$: DNA molar ratio, and the excess ratio of 20:1 $\mathrm{Ag}^{+}$: DNA for full i-motif 2 and 10:1 $\mathrm{Ag}^{+}$: DNA for half i-motif 2 . A standard growth assay was prepared using a 2-fold dilution series of the selected AgiMs from 0.5 $\mu \mathrm{M}-0.0075 \mu \mathrm{M}$ with P. aeruginosa strain PA01. Turbidity was measured at $600 \mathrm{~nm}$, with readings every 5 mins for 20 hours. The inhibitory effect of AgiMs was evaluated using three metrics, (1) the increase in apparent lag time to assess fractional killing of cells, (2) the area under the curve to assess accumulated biomass and (3) the turbidity at experiment completion to determine MIC. The antibacterial activity of the $6: 1 \mathrm{Ag}^{+}$: DNA prepared AgiMs was compared to the excess $\mathrm{Ag}^{+}$: DNA prepared AgiMs in Figure 3.1 (full i-motif 2) and Figure 3.2 (half i-motif 2).

Figure 3.1 compares the antibacterial activity of full i-motif 2 prepared with the standard and excess molar ratios. The growth curve in Figure 3.1a shows the growth profiles for both the 6:1 molar ratio (dotted lines) and the 20:1 molar ratio (solid lines) demonstrating a concentration-dependent killing effect with increasing concentrations of the AgiMs resulting in a proportional increase in the apparent lag time. The delay in growth is demonstrated by right-shifted parallel growth curves compared to the 
untreated control (black), with the 20:1 molar ratio curves showing increased growth inhibition compared to the 6:1 molar ratio curves (Figure 3.1a). At the completion of the experiment, no growth was detected in the $6: 1$ molar ratio full i-motif 2 at $0.5 \mu \mathrm{M}$, compared to the 20:1 molar ratio full i-motif 2 at $0.25 \mu \mathrm{M}$. The MIC was recorded as 0.5 $\mu \mathrm{M}$ and $0.25 \mu \mathrm{M}$ respectively, demonstrating a 2-fold decrease in MIC when the excess molar ratio is used.

To assess the impact of treatment on the accumulated biomass the bacteria, area under the curve (AUC) was calculated and graphed. These data show that all concentrations of 20:1 full i-motif 2 significantly decreased the biomass compared to the control $(\mathrm{p}<0.001)$ (Figure 3.1c). Comparatively, the 6:1 full i-motif 2 only showed significance at concentrations $0.5 \mu \mathrm{M}, 0.25 \mu \mathrm{M}, 0.125 \mu \mathrm{M}$ and $0.06 \mu \mathrm{M}(\mathrm{p}<0.01)$ (Figure 3.1c).

The strength of antimicrobial activity for the two molar ratios was assessed by doseresponse curves, calculated by the difference in time for treatments to reach a turbidity threshold of $\mathrm{OD}_{600}=0.1$, compared to the control. IC 50 values were determined using a non-linear regression model with log (inhibitor) vs normalised response (variable slope). The IC 50 value for $6: 1$ full i-motif 2 was $0.1538 \mu \mathrm{M}$ (95 \%CI: $0.1198 \mu \mathrm{M}$ to $0.1974 \mu \mathrm{M}$ ) and for $20: 1$ full i-motif 2 was $0.0844 \mu \mathrm{M}(95 \% \mathrm{CI}: 0.0680 \mu \mathrm{M}$ to $0.1048 \mu \mathrm{M})$. Notably, this shows the 20:1 molar ratio resulted in an almost 2-fold decrease in IC50 value compared to the $6: 1 \mathrm{Ag}^{+}$ratio, consistent with improved antibacterial activity.

Figure 3.2 compares the antibacterial activity of half i-motif 2 prepared with the standard and excess molar ratios. The growth curve in Figure 3.2a shows the growth profiles for both the 6:1 molar ratio (dotted lines) and the 10:1 molar ratio (solid lines) demonstrating a concentration-dependent killing effect with increasing concentrations of the AgiMs resulting in a proportional increase in the apparent lag time. The delay in 
growth is demonstrated by right-shifted parallel growth curves compared to the untreated control (black), with the 10:1 molar ratio demonstrating a small increase in growth inhibition compared to the 6:1 molar ratio curves (Figure 3.2a).

These data show that the excess $\mathrm{Ag}^{+}$ratio preparation resulted in increased antibacterial activity compared to the $6: 1$ ratio preparation of half i-motif 2 ; however, this effect was not as significant as seen in full i-motif 2 . At the completion of the experiment, no growth was detected at $0.5 \mu \mathrm{M}$ in both molar rations, as such, the MIC was recorded as $0.5 \mu \mathrm{M}$.

AUC was calculated to assess the effect of treatment on the accumulated biomass. These data show all concentrations of half i-motif 2 in both 6:1 molar ratio, and 10:1 molar ratio were significantly different to the untreated control $(\mathrm{p}<0.001)$ (Figure 3.2c, 3.2c.1).

The antibacterial activity was further assessed by analysis of dose-response curves calculated by the difference in time for treatments to reach a turbidity threshold of $\mathrm{OD}_{600}=0.1$, compared to the control. The $\mathrm{IC}_{50}$ value for the $6: 1$ half $\mathrm{i}$-motif 2 was 0.1458 $\mu \mathrm{M}$ (95 \%CI: $0.0976 \mu \mathrm{M}$ to $0.2175 \mu \mathrm{M})$, compared to an IC50 value of $0.1666 \mu \mathrm{M}(95 \% \mathrm{CI}$ : $1409 \mu \mathrm{M}$ to $0.1970 \mu \mathrm{M}$ ) for the $10: 1$ half $\mathrm{i}$-motif 2 . These data indicate a similar antibacterial activity with similar IC 50 values (Figure $3.2 \mathrm{~b}, 3.2 \mathrm{~b} .1$ ).

Overall, these findings confirm the hypothesis that the 6:1 molar ratio was not fully saturating the full i-motif with $\mathrm{Ag}^{+}$, which limited the antibacterial activity. The $\mathrm{IC}_{50}$ values of the full -motif and half -motif at the 6:1 molar ratio were comparable, at 0.1538 $\mu \mathrm{M}$ and $0.1458 \mu \mathrm{M}$ respectively. The antibacterial activity of both AgiMs improved using the excess $\mathrm{Ag}^{+}$: DNA molar ratios, in particular the full i-motif which exhibited an almost 2 -fold increase in antibacterial activity. The $\mathrm{IC}_{50}$ values of the full -motif and half -motif at the excess molar ratio were $0.08444 \mu \mathrm{M}$ and $0.1666 \mu \mathrm{M}$ respectively. 


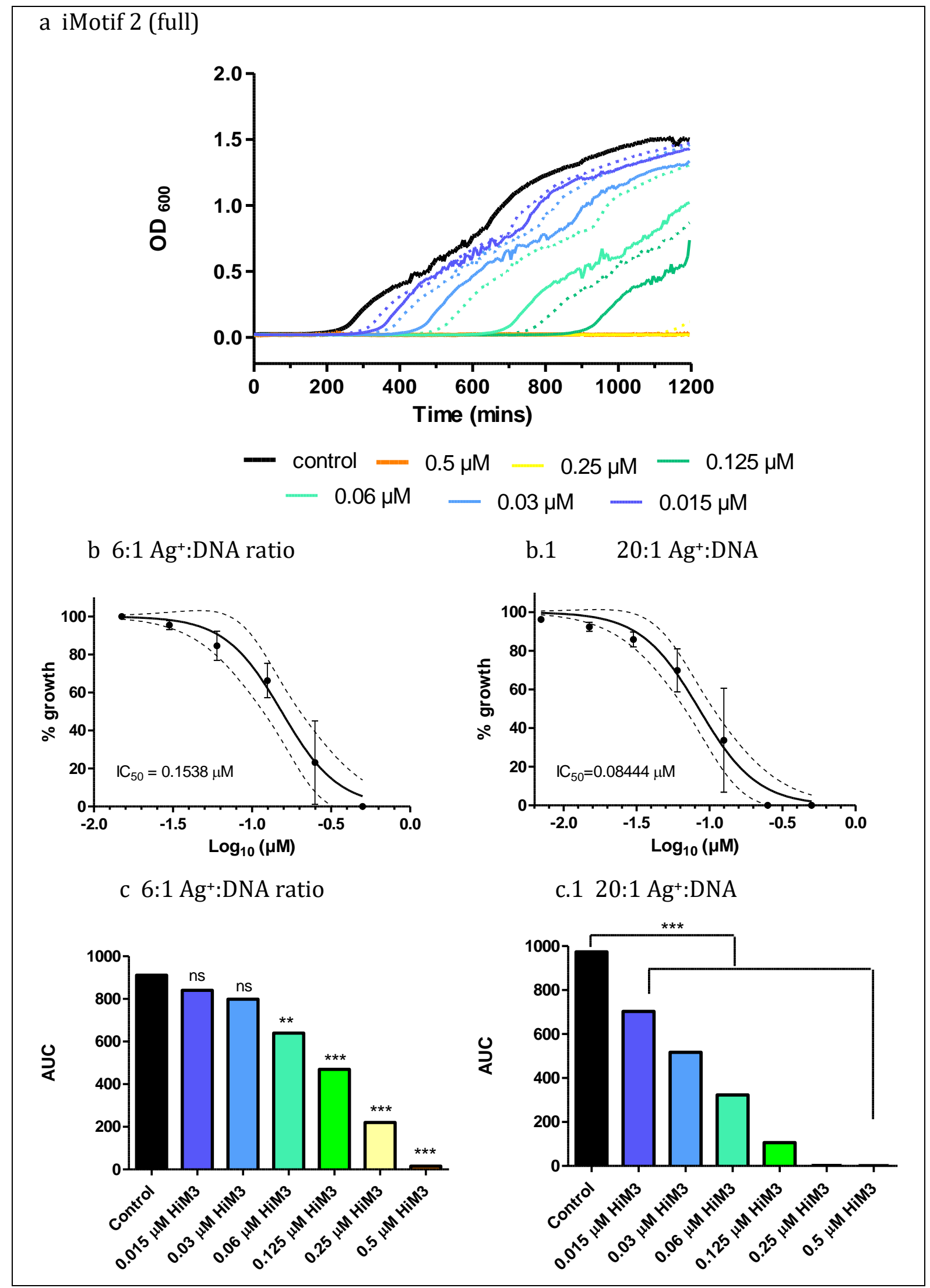

Figure 3.1 Comparison of 6:1 ratio with excess $\mathrm{Ag}^{+}$ratio in Full i-motif 2 .

PA01 was treated with a 2-fold dilution series of 6:1 $\mathrm{Ag}^{+}$: DNA full i-motif 2 (dotted lines) or 20:1 $\mathrm{Ag}^{+}$: DNA full i-motif 2 (solid lines) and the growth measured by turbidity at $600 \mathrm{~nm}$ every 5 minutes for 20-h. The growth curves represent three independent experiments (a). 
The growth curves from Figure a were used to calculate the difference in the time to reach $\mathrm{OD}_{600}=0.1$ compared to the untreated control. The results were plotted as a normalised response (\% growth) versus log concentrations. The IC 50 values were determined using a non-linear regression model with log (inhibitor) vs normalised response (variable slope). The dots represent the mean of three independent experiments with error bars representing SD, the solid lines show the fitted non-linear regression curve, and the dotted lines show the $95 \%$ confidence interval of the non-linear regression curve for $6: 1$ Ag+: DNA full i-motif 2 (b) and 20:1 Ag+: DNA full i-motif 2 (b.1). The area under the curve (AUC) of each growth profile was calculated and graphed. The bars represent three independent experiments for 6:1 $\mathrm{Ag}^{+}$: DNA full i-motif 2 (c) and 20:1 $\mathrm{Ag}^{+}$: DNA full i-motif 2 (c.1). The p values were calculated using one-way ANOVA with Dunnets post-hoc test to compare all treatments to the control. ${ }^{* * *} \mathrm{p}<0.001,{ }^{* *} \mathrm{p}<0.01, \mathrm{~ns}=$ no significance 


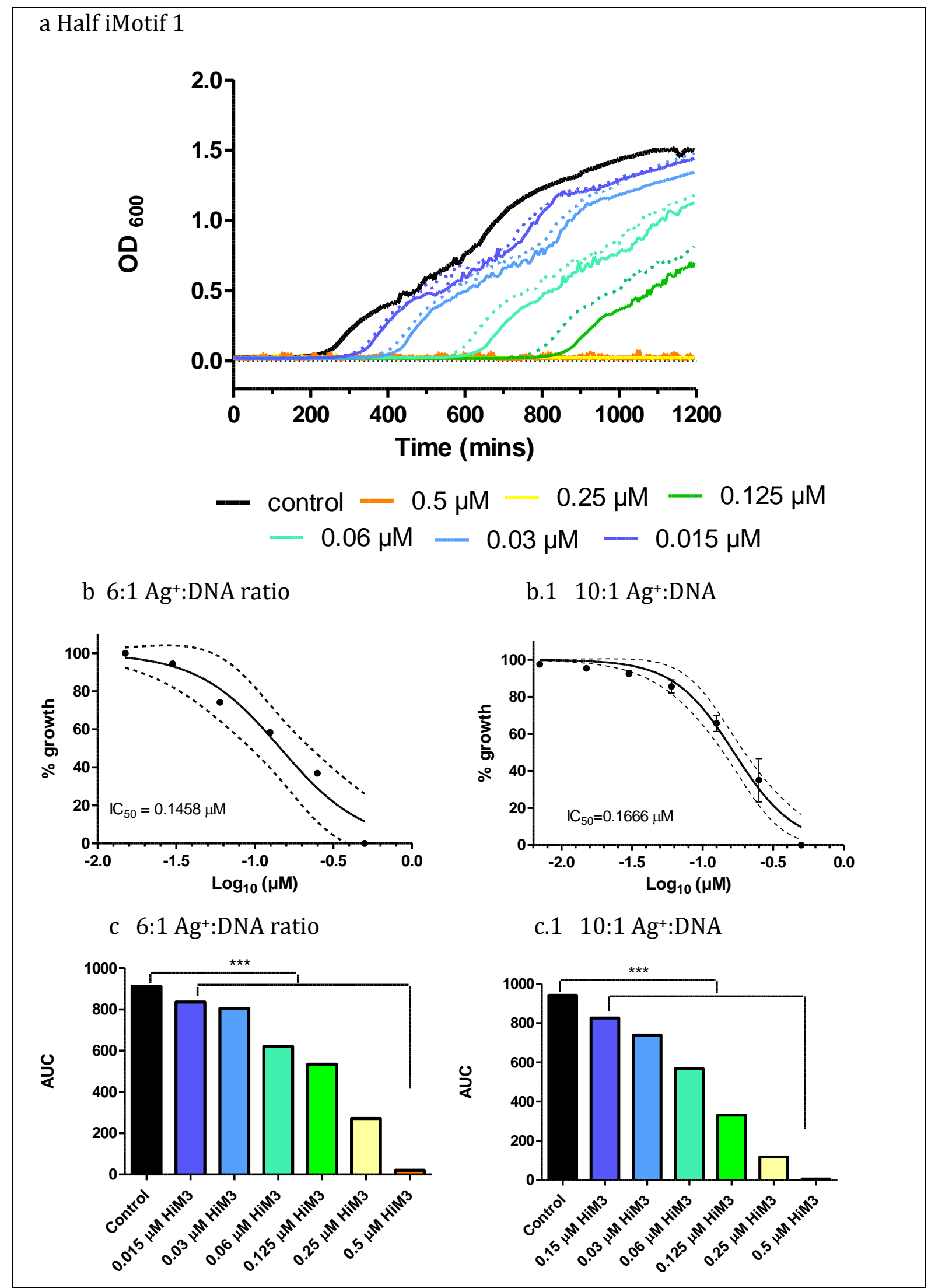

Figure 3.2 Comparison of 6:1 Ag:DNA molar ratio with 10:1 Ag:DNA in Half i-motif 2.

PA01 was treated with a 2-fold dilution series of 6:1 Ag+: DNA half i-motif 2 (dotted lines) or 10:1 Ag+: DNA half i-motif 2 (solid lines) and the growth measured by turbidity at $600 \mathrm{~nm}$ every 5 minutes for 20-h. The growth curves represent three independent experiments (a). The growth 
curves from Figure a were used to calculate the difference in the time to reach $\mathrm{OD}_{600}=0.1$ compared to the untreated control. The results were plotted as a normalised response (\% growth) versus $\log$ concentrations. The $\mathrm{IC}_{50}$ values were determined using a non-linear regression model with log (inhibitor) vs normalised response (variable slope). The dots represent the mean of three independent experiments with error bars representing SD, the solid lines show the fitted non-linear regression curve, and the dotted lines show the 95\% confidence interval of the non-linear regression curve for 6:1 Ag+: DNA half i-motif 2 (2b) and 10:1 $\mathrm{Ag}^{+}$: DNA half i-motif 2 (b.1). The area under the curve (AUC) of each growth profile was calculated and graphed. The bars represent three independent experiments for 6:1 $\mathrm{Ag}^{+}$: DNA half i-motif 2 (c) and 10:1 $\mathrm{Ag}^{+}$: DNA half i-motif 2 (c.1). The p values were calculated using one-way ANOVA with Dunnets posthoc test to compare all treatments to the control. ${ }^{* * *} \mathrm{p}<0.001$ 


\subsubsection{The poly-C region of AgiMs associated with antimicrobial potency}

The i-motif sequences designed for this study varied in poly-C length, with identical nucleotide turn regions (TAA). It was hypothesised that all sequences would form silverstabilised i-Motifs, with increasing poly-C regions correlated to increasing antimicrobial potency. It was expected that antimicrobial potency would increase proportionally to an increase in the length of the poly-C region for each sequence.

To investigate this hypothesis, a standard growth assay was performed using the three intra-strand full i-motifs and three inter-strand dimeric half i-motifs in a concentration range of $0.5 \mu \mathrm{M}$ to $0.075 \mu \mathrm{M}$. The antibacterial activity was assessed as earlier, by three metrics (1) the increase in apparent lag time to assess fractional killing of cells, (2) the area under the curve to assess accumulated biomass and (3) the turbidity at experiment completion to determine MIC.

As expected, all AgiMs exhibited concentration-dependent growth inhibition with increasing concentrations of AgiMs, resulting in a proportional increase in the apparent lag time (Figure 3.3). This effect is demonstrated by the right-shifted parallel growth curves compared to the untreated control. The parallel curves indicate AgiMs rapidly kill a fraction of the inoculum, consistent with a reduction in the inoculum size without changing the growth rate of surviving cells. No growth was detected in the $0.5 \mu \mathrm{M}$ treatment of half i-motif 1 , half i-motif 2 and half i-motif 3 at the completion of the experiment; as such, the MIC was recorded as $0.5 \mu \mathrm{M}$. Full i-motif 1 , full i-motif 2 and full i-motif 3 had no growth for $0.5 \mu \mathrm{M}$ and $0.25 \mu \mathrm{M}$ at experiment completion; as such, the MIC was recorded as $0.25 \mu \mathrm{M}$. 
Analysis of AUC for each growth profile showed all AgiM treatments significantly reduced the accumulated biomass of the inoculum, with statistical significance achieved even at the lowest concentration $0.0075 \mu \mathrm{M}(\mathrm{p}<0.001)$ (Figure 3.5).

The strong antimicrobial activity of the AgiMs was confirmed by analysis of doseresponse curves as described earlier. The $\mathrm{IC}_{50}$ values for half i-motif 1 , half $\mathrm{i}$-motif 2 , half i-motif 3, full i-motif 1, full i-motif 2 and full i-motif 3 were $0.1655 \mu \mathrm{M}$ (95 \%CI: 0.1237 to 0.2214), $0.1666 \mu \mathrm{M}$ (95 \%CI: 0.14 to 0.1970$), 0.1378$ (95 \%CI: 0.1198 to 0.1586 ), 0.07820 $\mu \mathrm{M}$ (95 \%CI: 0.06921 to 0.08837 ), $0.08444 \mu \mathrm{M}$ (95 \%CI:0.06803 to 0.1048 ), $0.07059 \mu \mathrm{M}$ (95 \%CI:0.05830 to 0.08547 ) respectively (Figure 3.4 ). These data indicate a strong antibacterial activity for all AgiMs tested, with increasing $\mathrm{C}^{-\mathrm{C}^{+}}$base pairs correlated with a decrease in $\mathrm{IC}_{50}$ values.

Table 3-1 shows a summary of the results from this experiment, with the MIC and IC50 values of each AgiM listed. This table shows the increasing $\mathrm{C}^{-\mathrm{C}^{+}}$base pair number correlates with a decrease in MIC and IC50. Overall, these findings support the hypothesis that the $\mathrm{C}-\mathrm{C}^{+}$base pair number is directly proportional to the antibacterial activity.

\begin{tabular}{|c|c|c|c|}
\hline i-Motif & $\mathrm{C}-\mathrm{C}+$ & MIC & $\mathrm{IC}_{50}(95 \% \mathrm{CI})$ \\
\hline Half i-motif 1 & 3 & $0.5 \mu \mathrm{M}$ & $\begin{array}{c}0.1655 \mu \mathrm{M} \\
(0.1237 \mu \mathrm{M} \text { to } 0.2214 \mu \mathrm{M})\end{array}$ \\
\hline Half i-motif 2 & 4 & $0.5 \mu \mathrm{M}$ & $\begin{array}{c}0.1666 \mu \mathrm{M} \\
(0.1409 \mu \mathrm{M} \text { to } 0.1970 \mu \mathrm{M})\end{array}$ \\
\hline Half i-motif 3 & 5 & $0.5 \mu \mathrm{M}$ & $\begin{array}{c}0.1378 \mu \mathrm{M} \\
(0.1198 \mu \mathrm{M} \text { to } 0.1586 \mu \mathrm{M})\end{array}$ \\
\hline Full i-motif 1 & 6 & $0.25 \mu \mathrm{M}$ & $\begin{array}{c}0.07820 \mu \mathrm{M} \\
(0.06921 \mu \mathrm{M} \text { to } 0.08837 \mu \mathrm{M})\end{array}$ \\
\hline Full i-motif 2 & 8 & $0.25 \mu \mathrm{M}$ & $\begin{array}{c}0.08444 \mu \mathrm{M} \\
(0.06803 \mu \mathrm{M} \text { to } 0.1048 \mu \mathrm{M})\end{array}$ \\
\hline Full i-motif 3 & 10 & $0.25 \mu \mathrm{M}$ & $\begin{array}{c}0.07059 \\
(0.05830 \mu \mathrm{M} \text { to } 0.08547 \mu \mathrm{M})\end{array}$ \\
\hline
\end{tabular}




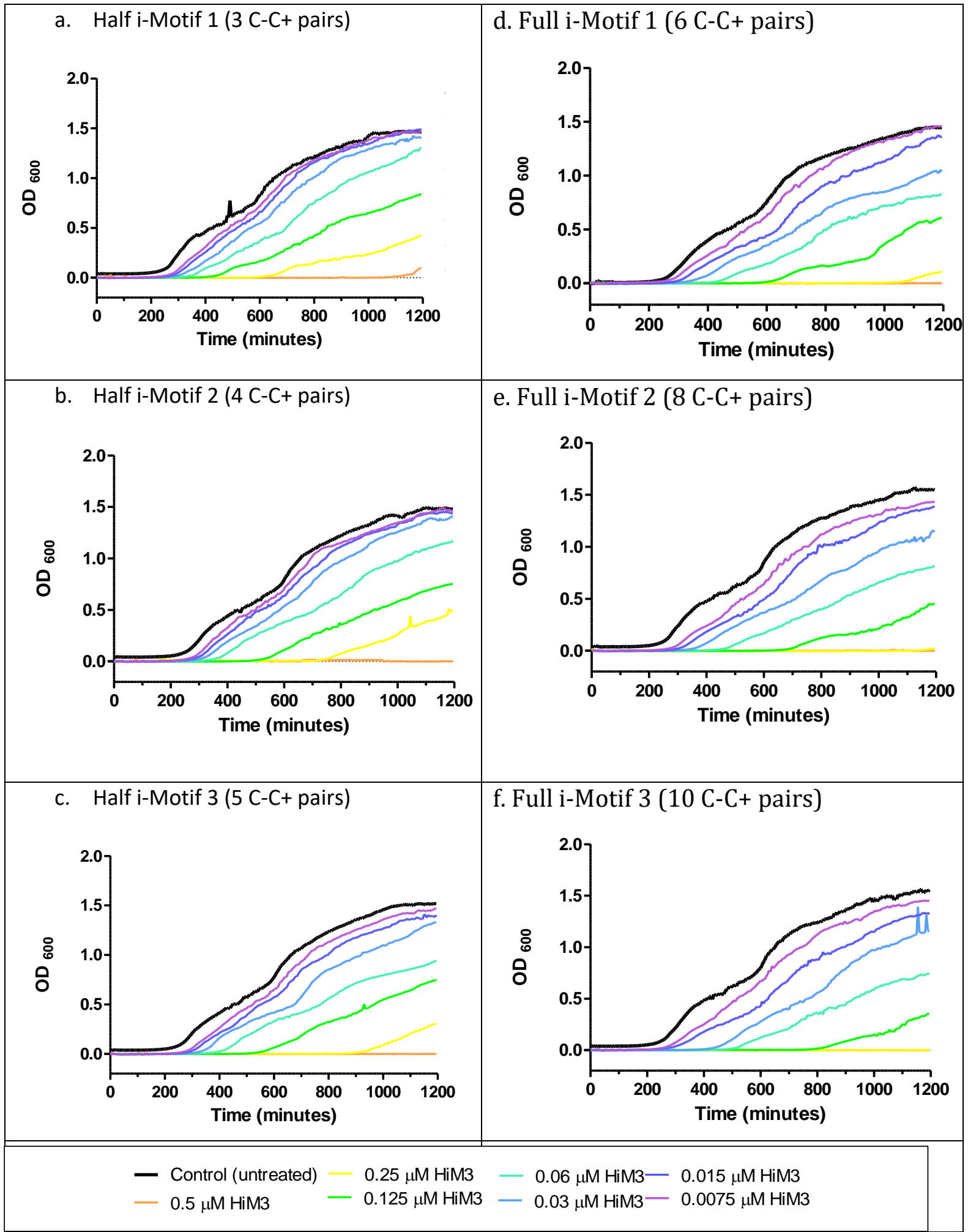

Figure 3.3 The number of $\mathrm{C}-\mathrm{C}+\mathrm{bp}$ is proportional to the antibacterial activity of AgiMs

. PA01 was treated with a 2-fold dilution series of half i-motif 1 (A), half i-motif 2 (B), half i-motif 3 (C), full i-motif 1 (D), full i-motif 2 (E), full i-motif $3(\mathrm{~F})$ in a concentration range of $0.5 \mu \mathrm{M}$ to $0.075 \mu \mathrm{M}$. Growth measured by turbidity at $600 \mathrm{~nm}$ every 5 minutes for 20 -h. The growth curves represent three independent experiments. 


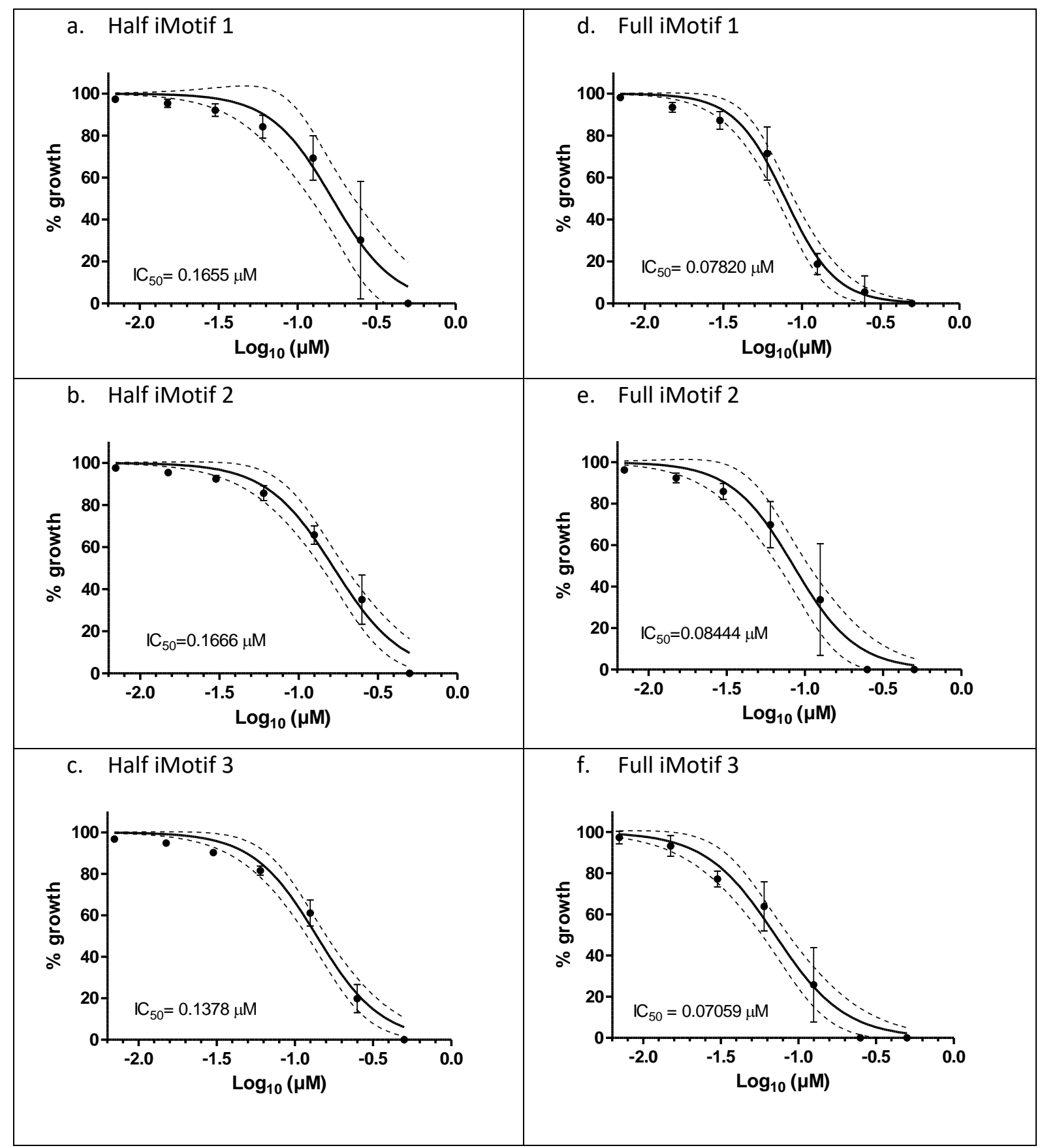

Figure 3.4 Dose-response curves demonstrate AgiM exhibit strong antibacterial activity.

Dose-response curves were calculated from difference to time to the threshold (OD600 $=0.1$ ) compared to the control. Results plotted as \% growth vs log concentration. IC50 values were determined using non-linear regression for log (inhibitor) vs normalised response (variable slope). The dots represent the mean of three independent experiments with error bars representing SD, the solid lines show the fitted non-linear regression curve, and the dotted lines show the $95 \%$ confidence interval of the non-linear regression curve 


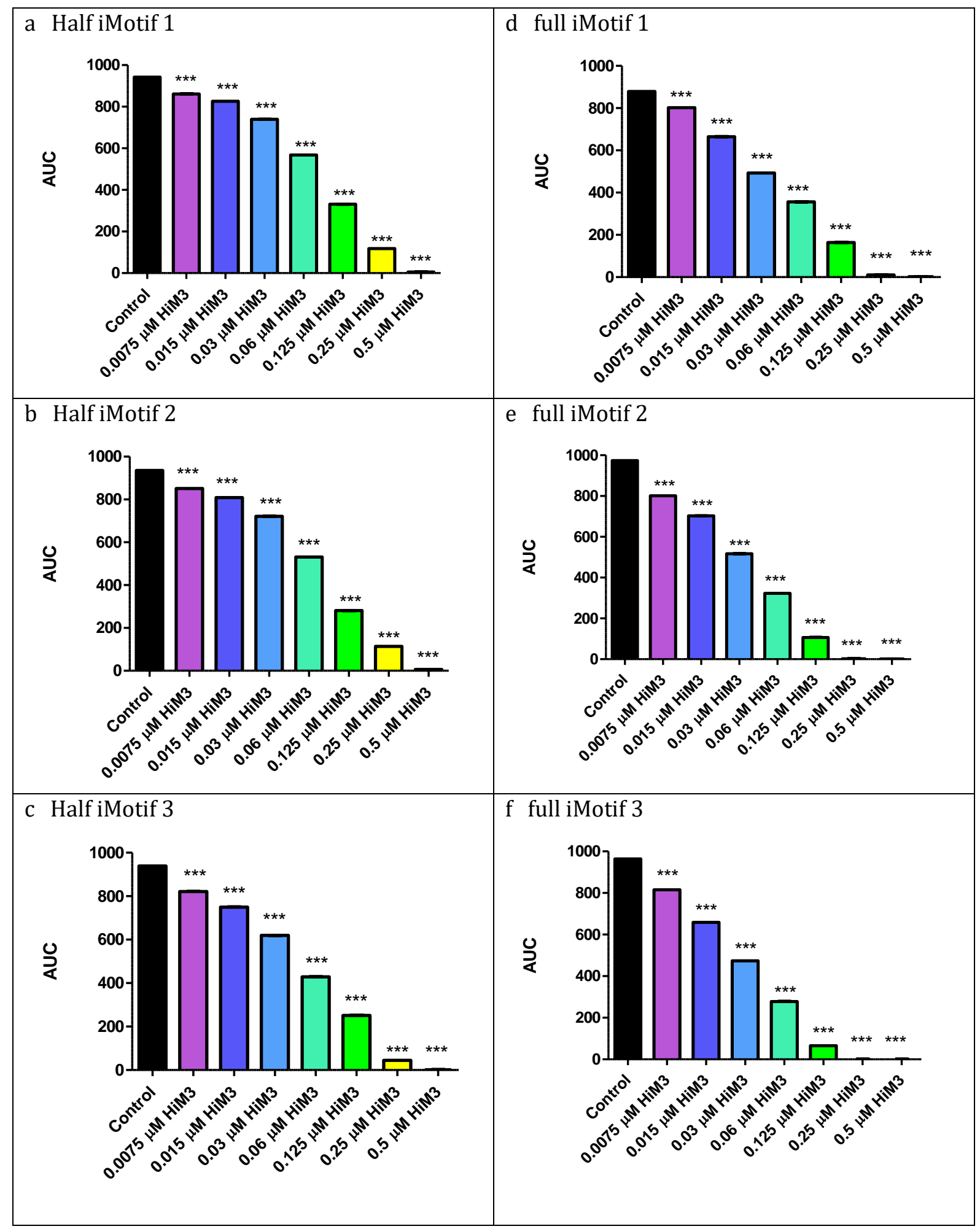

Figure 3.5 Antibacterial activity:

3.5a-f: The area under the curve (AUC) of each growth profile was calculated and graphed. The bars represent three independent experiments for 6:1 $\mathrm{Ag}^{+}$: DNA full i-motif 2 (1c) and 20:1 $\mathrm{Ag}^{+}$: DNA full i-motif 2 (1c.1). The p values were calculated using one-way ANOVA with Dunnets posthoc test to compare all treatments to the control. ${ }^{* * *} \mathrm{p}<0.001$ 


\subsection{Key findings}

The experiments in this chapter aimed to determine the relationship between i-motif sequence and antibacterial activity. It is predicted that the number of $\mathrm{C}-\mathrm{C}^{+}$base pairs in each sequence is directly proportional to the silver content and resulting antibacterial activity.

Initial experiments confirmed an excess $\mathrm{Ag}^{+}$: DNA molar ratio ensured sufficient silver saturation during AgiM preparation and resulted in enhanced antibacterial activity. As expected, the full i-motif demonstrated a significant improvement in concentrationdependent growth inhibition and resulted in a 2 -fold decrease in recorded MIC and $\mathrm{IC}_{50}$ values, consistent with enhanced antibacterial activity. The half i-motif showed minor improvement in growth inhibition, with MIC and IC $_{50}$ values remaining the same. These findings show the 6:1 molar ratio was not sufficient for the full i-motif sequence which is capable of binding twice as much silver than the half i-motif. These data also support the hypothesis that full i-motifs are twice as effective as their half i-motif counterparts on a molar basis.

A comparison of the number of $\mathrm{C}_{-} \mathrm{C}^{+}$base pairs with the antibacterial activity observed in the AgiM sequences tested in this chapter was made in Figure 6, using the time to reach a threshold of $\mathrm{OD}_{600}=0.1$ to calculate bacterial growth delay. If the $\mathrm{C}^{-\mathrm{C}^{+}}$number was directly proportional to the silver content (as the results in this chapter suggest), it would be expected that this graph would show both $\mathrm{C}^{-\mathrm{C}^{+}}$number and growth delay increasing proportionally to each other. Unexpectedly, growth delay increases from $3 \mathrm{C}^{-\mathrm{C}^{+}}$bp to up $6 \mathrm{C}^{-\mathrm{C}^{+}} \mathrm{bp}$, but then plateaus with no increase in growth delay over $8 \mathrm{C}-\mathrm{C}^{+}$bp and $10 \mathrm{C}-\mathrm{C}^{+}$ bps. These data indicate high sensitivity across the half i-motifs with antibacterial activity proportional to the $\mathrm{C}_{-} \mathrm{C}^{+}$number, conversely lower sensitivity is observed across the full 
i-motifs with growth delayed plateaued. This discrepancy suggests other mechanisms may be involved in the correlation between AgiM sequence and silver content. This could be due to the variety of i-motif configurations, and steric constraints which may limit the amount of silver able to bind. There is a correlation between $\mathrm{C}^{-} \mathrm{C}^{+} \mathrm{bp}$ number and silver content in AgiMs, but it may not be a linear one.

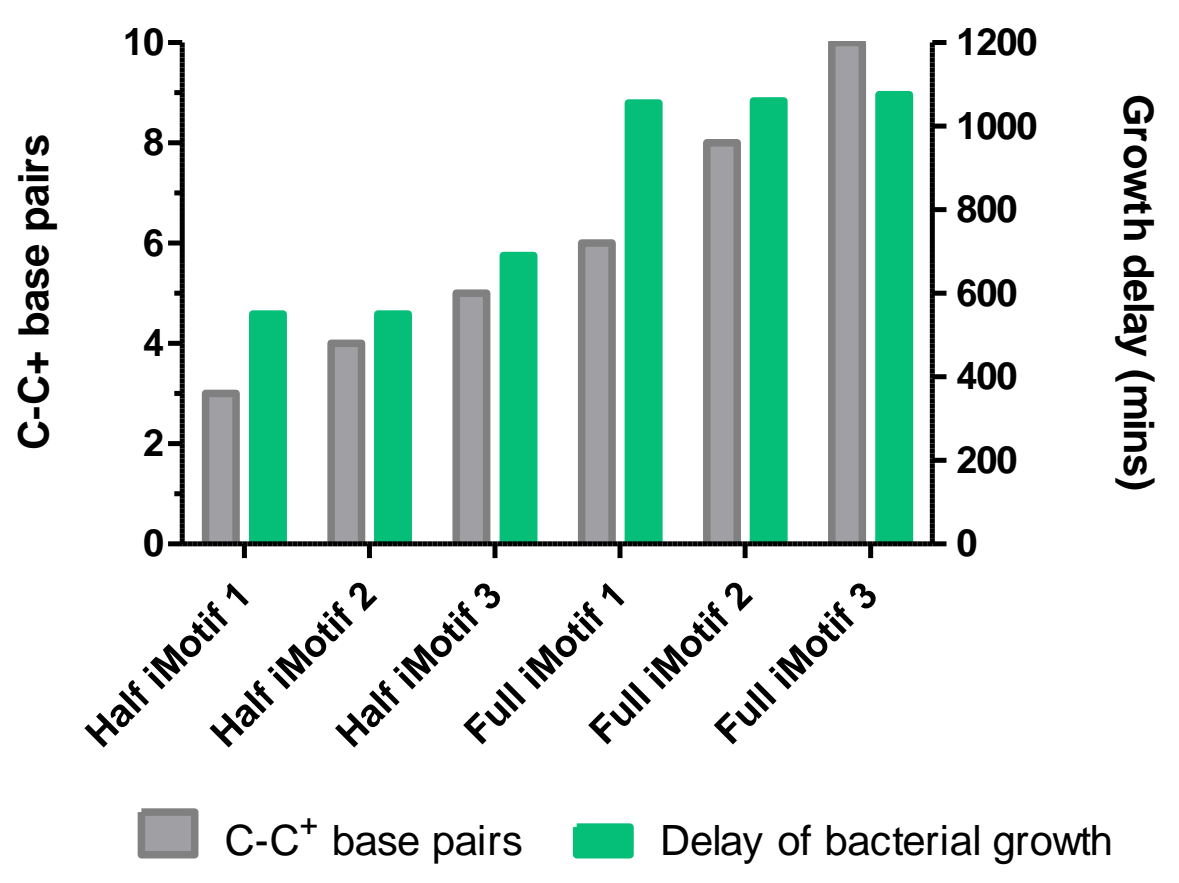

Figure 3.6 Comparison of iMotif sequence C-C+ pair number to corresponding bacterial growth inhibiton.

PA01 was treated with $0.125 \mu \mathrm{M}$ of each AgiM variation (Half iMotif 1, Half iMotif 2, Half iMotif 3 , iMotif 1 , iMotif 2, iMotif 3 ). The delay in bacterial growth (right y-axis) represents time to reach threshold (OD600 $=0.1$ ) compared to the $\mathrm{C}-\mathrm{C}+$ base pair number (left $\mathrm{y}$-axis).

A candidate i-motif sequence was selected to use throughout the remainder of this study.

Based on the findings of this chapter, half i-motif sequences displayed strong antibacterial activity and high sensitivity killing of $P$. aeruginosa. These sequences present a commercially viable and therapeutically relevant option. Synthesis of smaller oligonucleotides sequences is more financially viable, and contain a low silver content reducing potential toxicity. Half i-motif 3 (TCCCCCTAACCCCCT) was selected as the 
candidate sequence, displaying the strongest growth inhibition of the three half i-motif sequences.

AgiMs provide a novel method to deliver soluble $\mathrm{Ag}^{+}$to bacteria, demonstrating concentration-dependent antibacterial activity against PA01 strain of $P$. aeruginosa. The i-motif structure allows the delivery of $\mathrm{Ag}^{+}$without the nitrate groups associated with $\mathrm{AgNO}_{3}$. The antibacterial activity of AgiMs will be further explored and discussed in Chapter 4, using half i-motif 3 (HiM3) as the candidate sequence. 


\subsection{Discussion}

The direct correlation between the poly-C tract and silver content in AgiMs is not fully understood. The binding mechanism has been described by Swasey and colleagues, with $\mathrm{Ag}^{+}$able to bind at the $\mathrm{N} 3$ atom of cytosine in $\mathrm{C}^{-} \mathrm{C}^{+}$base pair duplexes with hydrogen bonds aiding in stabilisation of the complex (69) (Figure 3.5). Because of this binding mechanism is was proposed that each $\mathrm{C}^{-} \mathrm{C}^{+}$pair is capable of binding one $\mathrm{Ag}^{+}$, indicating a direct correlation to poly-C length and antibacterial activity.

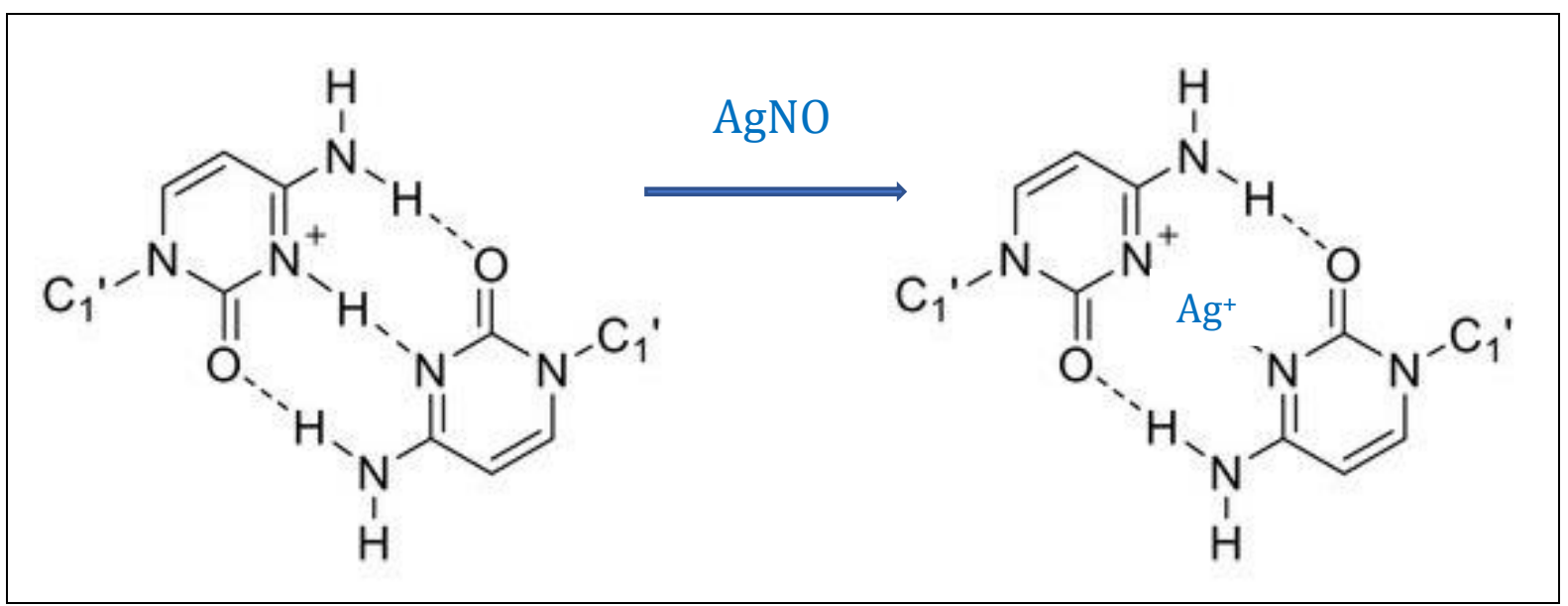

Figure 3.7 Schematic of proposed binding mechanism of $\mathrm{Ag}^{+}$in AgiM.

The addition of $\mathrm{AgNO}^{3}$ to the sequence induces the formation of AgiM. The $\mathrm{Ag}^{+}$binds the N3 atom of cytosine in $\mathrm{C}_{-} \mathrm{C}^{+}$base pair duplexes with hydrogen bonds aiding in stabilisation of the complex (Swasey et al., 2015). Figure adapted from (Abdelhamid et al., 2018) with permission from Oxford University Press, Copyright (C) 2018

The results of the chapter partially support this; however, it is evident other factors are involved as the relationship between $\mathrm{C}^{-\mathrm{C}^{+}}$bp number, and bacterial growth delay was not linear.

The unique conformations of silver-stabilised i-motif structures are still being investigated. Day and colleagues reported the indicated stoichiometry of the silverstabilised i-motif for the hTeloC sequence was less than expected. Based on CD analysis, the structures had a 4:1 $\mathrm{Ag}^{+}$: DNA molar ratio which was less than the potential $\mathrm{Ag}^{+}$ binding positions indicated by the sequence. This suggests silver-stabilised i-motif 
structures could be composed of a mixture of $\mathrm{C}: \mathrm{Ag}^{+}: \mathrm{C}^{+}$and $\mathrm{C}: \mathrm{H}^{+}: \mathrm{C}^{+}$and that the $4: 1 \mathrm{Ag}^{+}$: DNA stoichiometry observed could indicate the minimal amount of silver cations required to induce i-motif folding (66).

A complete physical characterisation of the AgiMs to precisely determine the number of silver atoms on each oligonucleotide would be beneficial to allow accurate correlation of silver atoms and antibacterial activity. Unfortunately due to time constraints silver quantification of AgiMs was not achieved in this study. 



\section{CHAPTER 4. INVESTIGATION OF AGIMS IN VITRO}

\section{ANTIBACTERIAL ACTIVITY}

\subsection{Aims and rationale}

The broad-spectrum antimicrobial activity of silver is well established, with an increasing number of applications using silver and AgNPs in both consumer and clinical products (48). Silver stabilised i-motifs (AgiMs) offer a soluble $\mathrm{Ag}^{+}$delivery system, which hold antibacterial therapeutic potential. The experiments performed in this chapter aim to investigate the antibacterial activity of AgiM HiM3 against $P$. aeruginosa, as well as provide preliminary data on its activity against $A$. baumannii. Antimicrobial susceptibility testing is a common and crucial step in drug discovery; used to predict therapeutic outcome and determine the potential of antimicrobial agents (44).

To examine the antibacterial activity of AgiM HiM3 standard growth assays were performed on exponentially growing inoculums of $P$. aeruginosa and A. baumannii. Both of these Gram-negative bacteria are known to evade antimicrobial treatment, resulting from resistance mechanisms conferring multi-drug resistance. All other experiments continued with P. aeruginosa as the test pathogen, using time-to-kill kinetics to determine preliminary pharmacokinetic information; followed by the investigation of AgiMs effect on pyoverdine production. Pyoverdine is a siderophore produced by $P$. aeruginosa associated with the virulence of infection. It sequesters iron from the environment and is able to intercept it from host iron-sequestering proteins transferrin and lactoferrin (27). Recent reports have indicated interfering with bacterial iron homeostasis, by inhibition of pyoverdine production may serve as a potential therapeutic target (25). 
P. aeruginosa possesses several innate defence mechanisms including the expression of four major efflux pumps (MexAB-OprM, MexCD-OprJ, MexEF-OprnN and MexXY-OprM) that reduce intracellular drug concentrations, conferring resistance to several classes of antibiotic ( $\beta$-lactams, fluoroquinolones and aminoglycosides) (25). The final experiment aimed to determine the effect of AgiMs in the presence and absence of efflux pumps, to establish if strains could develop resistance to AgiM treatment.

\subsection{Bacterial strains antibiotic susceptibilities}

The antibiotic susceptibilities of the bacterial strains used in this study were determined using the Kirby-Bauer Disk Diffusion Protocol (as described in Methods) to determine the sensitivity or resistance to a variety of antibiotics, further described in Appendix B.

The resistance or sensitivity of each strain to the listed antimicrobials was determined by measuring the size of growth inhibition zones $(\mathrm{mm})$ on the surface of the culture. Measurements listed are the mean of duplicate LB and MH agar plates. Classifications were based on the American Society for Microbiology standards for Pseudomonas and other non-fermenting Gram-Negative Rod bacteria. $\mathrm{S}=$ sensitive, $\mathrm{I}=$ intermediate , $\mathrm{R}=$ resistant

\begin{tabular}{|c|c|c|c|c|c|c|}
\hline \multirow[t]{2}{*}{ Strain } & \multicolumn{6}{|l|}{ Antibiotics } \\
\hline & Tobramycin & Ciprofloxacin & Piperacillin & Cefotaxime & Tetracycline & Gentamicin \\
\hline PA01 & $24 \mathrm{~mm} \mathrm{~S}$ & $30 \mathrm{~mm} \mathrm{~S}$ & $31 \mathrm{~mm} \mathrm{~S}$ & $23 \mathrm{~mm} \mathrm{~S}$ & $10 \mathrm{~mm} \mathrm{R}$ & $20 \mathrm{~mm} \mathrm{~S}$ \\
\hline 1024 & $18 \mathrm{~mm} \mathrm{~S}$ & $26 \mathrm{~mm} \mathrm{~S}$ & $19 \mathrm{~mm} \mathrm{~S}$ & $0 \mathrm{~mm} \mathrm{R}$ & $5 \mathrm{~mm} \mathrm{R}$ & $13 \mathrm{~mm} \mathrm{I}$ \\
\hline 1079 & $21 \mathrm{~mm} \mathrm{~S}$ & $35 \mathrm{~mm} \mathrm{~S}$ & $28 \mathrm{~mm} \mathrm{~S}$ & $18 \mathrm{~mm} \mathrm{I}$ & $13 \mathrm{~mm} \mathbf{R}$ & $16 \mathrm{~mm} \mathrm{~S}$ \\
\hline 1205 & $19 \mathrm{~mm} \mathrm{~S}$ & $26 \mathrm{~mm} \mathrm{~S}$ & $20 \mathrm{~mm} \mathrm{~S}$ & $5 \mathrm{~mm} \mathrm{R}$ & $0 \mathrm{~mm} \mathbf{R}$ & $14 \mathrm{~mm} \mathrm{I}$ \\
\hline
\end{tabular}


Table 4-1 show all strains of $P$. aeruginosa tested were sensitive to Piperacillin - a broad spectrum $\beta$-lactam antibiotic, ciprofloxacin - a fluoroquinolone and tobramycin - an aminoglycoside antibiotic, with significant growth inhibition zones visualised on the agar. All strains showed resistance to Tetracycline, a broad-spectrum bacteriostatic antibiotic that is only effective against multiplying microorganisms. Clinical isolates 1024 and 1205 were resistant to cefotaxime; with PA01 and isolate 1079 sensitive and intermediate respectively to cefotaxime, a broad-spectrum antibiotic. Strains PA01 and 1079 were sensitive to Gentamicin, and clinical isolates 1024 and 1205 intermediate. In summary, none of the P. aeruginosa strains used in this study were classified as multi-drug resistant; defined as resistant to three or more classes of antibiotic.

\begin{tabular}{|lllllll|}
\hline \multicolumn{6}{l}{ Table 4-2 Antibiotic susceptibilities of A. baumannii strains used in this study } \\
\hline Strain & Antibiotics & & & & \\
\hline & Tobramycin & Ciprofloxacin & Piperacillin & Cefotaxime & Tetracycline & Gentamicin \\
\hline AB10 & $0 \mathrm{~mm} \mathbf{R}$ & $0 \mathrm{~mm} \mathbf{R}$ & $0 \mathrm{~mm} \mathbf{R}$ & $0 \mathrm{~mm} \mathbf{R}$ & $12 \mathrm{~mm} \mathbf{R}$ & $0 \mathrm{~mm} \mathbf{R}$ \\
\hline $\mathbf{A B 1 2}$ & $0 \mathrm{~mm} \mathbf{R}$ & $0 \mathrm{~mm} \mathbf{R}$ & $15 \mathrm{~mm} \mathbf{R}$ & $8 \mathrm{~mm} \mathbf{R}$ & $13 \mathrm{~mm} \mathbf{R}$ & $0 \mathrm{~mm} \mathbf{R}$ \\
\hline $\mathbf{A B 6}$ & $0 \mathrm{~mm} \mathbf{R}$ & $0 \mathrm{~mm} \mathbf{R}$ & $0 \mathrm{~mm} \mathbf{R}$ & $0 \mathrm{~mm} \mathbf{R}$ & $11 \mathrm{~mm} \mathbf{R}$ & $0 \mathrm{~mm} \mathbf{R}$ \\
\hline
\end{tabular}

Table 4-2 shows strain AB was susceptible to Ciprofloxacin, Piperacillin and Tetracycline with intermediate susceptibility to tobramycin and cefotaxime, and resistance only to Gentamycin an aminoglycoside. The three clinical strains tested, AB6, AB10 and AB12 were resistant to all antibiotics tested showing small inhibition zones and often no growth inhibition from the antibiotic disks. Multi-drug resistance is classified as resistance to three or more antibiotic classes. These clinical isolates of $A$. baumannii are classified as multi-drug resistant (MDR) as they are resistant to the five classes of antibiotics used in this study (Ureidiopenicillins, Aminoglycosides, Tetracyclines, $4^{\text {th }}$ Generation Cephalosporins and Fluoroquinolones). 


\subsection{Results}

\subsubsection{AgiM exhibit dose-dependent killing of $P$. aeruginosa}

The antimicrobial activity of AgiM HiM3 to three clinical isolates of $P$. aeruginosa was evaluated with standard growth assays using a 2-fold dilution series of AgiM, ranging from $0.015 \mu \mathrm{M}$ to $2 \mu \mathrm{M}$, and growth monitored for 20 -h at $37^{\circ} \mathrm{C}$.

As previously described in Chapter 3, AgiM HiM3 exhibited dose-dependent growth inhibition across all strains tested; increasing concentrations of HiM3 resulted in a proportional increase in the apparent lag time (Figure 4.1a-c). The delay in growth can be observed by the treatment growth curves that are right-shifted compared to the untreated control across all clinical isolates; consistent with a reduction in the inoculum size without changing the growth rate of surviving cells. No growth was detected in concentrations of $2 \mu \mathrm{M}$ and $1 \mu \mathrm{M}$ for all strains at the completion of the experiment, as such, the MIC was recorded as $1 \mu \mathrm{M}$.

Analysis of area under the curve (AUC) determined treatment with $0.015 \mu \mathrm{M}$ HiM3 caused a statistically significant decrease in accumulated biomass for strains 1024, 1205 $(\mathrm{p}<0.001)$ and $1079(\mathrm{p}<0.01)$ (Figure 4.1a-c). It is important to note that while statistical significance was achieved at low concentrations of AgiM, the overall decrease in biomass is small. To achieve $>50 \%$ decrease in biomass compared to control in strain 1079 required treatment of $0.25 \mu \mathrm{M}$, and strains 1205 and 1024 at $0.125 \mu \mathrm{M}$.

The strong antimicrobial activity of AgiM was confirmed by analysis of dose-response curves as described earlier. The IC 50 values of HiM3 in different strains of P. aeruginosa were determined to be $0.1042 \mu \mathrm{M}$ (95 \% CI: 0.09199 to 0.1181 ) for isolate $1205,0.1388$ $\mu \mathrm{M}(95 \% \mathrm{CI}: 0.11105$ to 0.1743$)$ in isolate 1024 and $0.1858 \mu \mathrm{M}$ (95 \%CI:0.1522 to 0.2268$)$ for isolate 1079 (Figure 4.2a-c) 


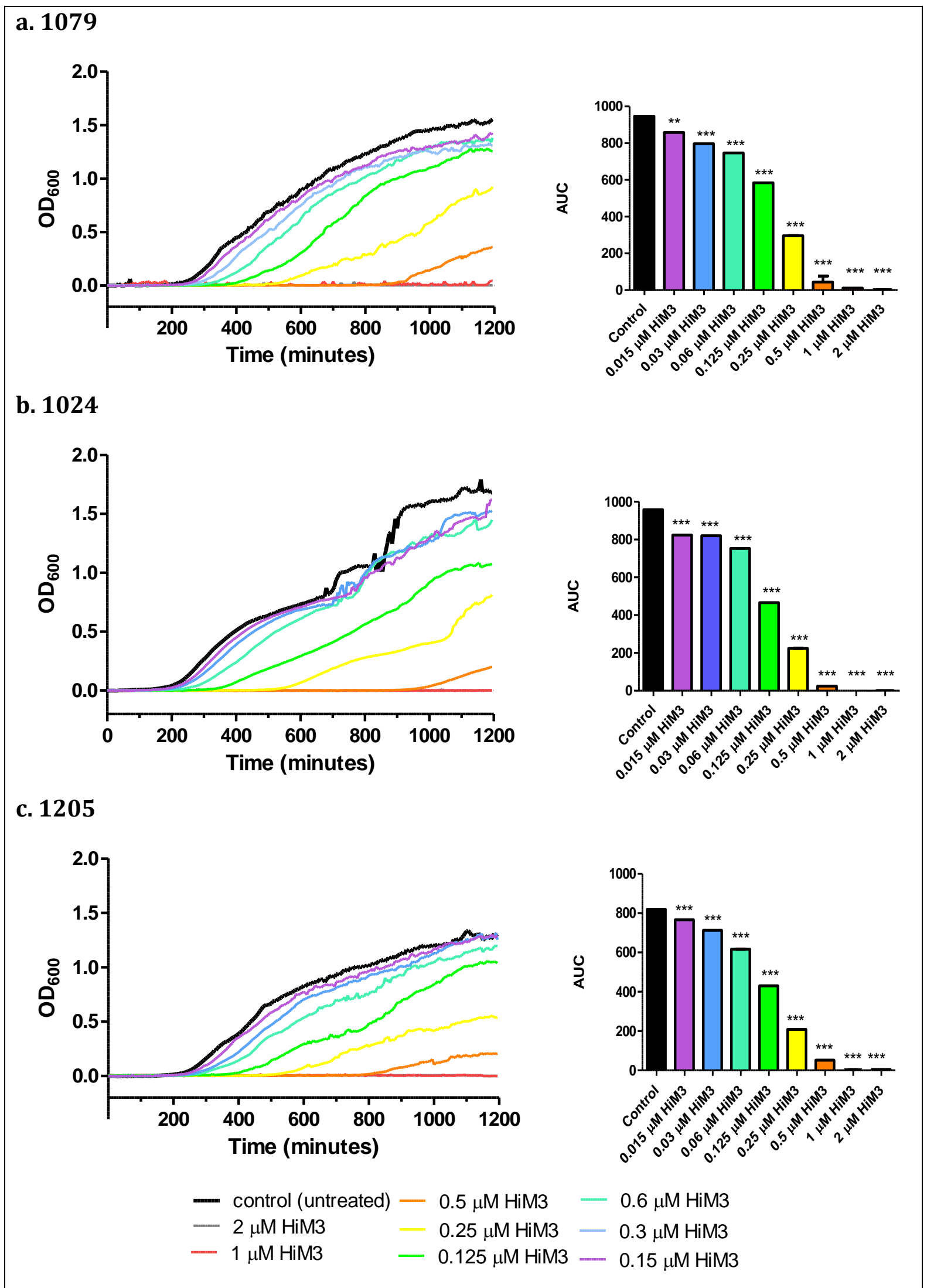

Figure 4.1a-c Dose-dependent antibacterial effect of AgiM HiM3.

P. aeruginosa clinical isolates 1079, 1205, 1024 were treated with a 2-fold dilution series of AgiM HiM3 at a concentration range of $2 \mu \mathrm{M}$ to $0.15 \mu \mathrm{M}$. Growth was monitored by turbidity at $600 \mathrm{~nm}$ every 5 minutes for 20 -h. The growth curves represent the mean of three independent 
experiments (left figures). The area under the curve (AUC) was calculated for each growth curve. The bar graphs represent the mean of three independent experiments, and the error bars show SD(right figures). P values were calculated using one-way ANOVA with Dunnet's post-hoc test to compare all means to the control. ${ }^{*} \mathrm{p}<0.05,{ }^{* *} \mathrm{p}<0.01, \mathrm{p}<0.001$ 
a. 1079

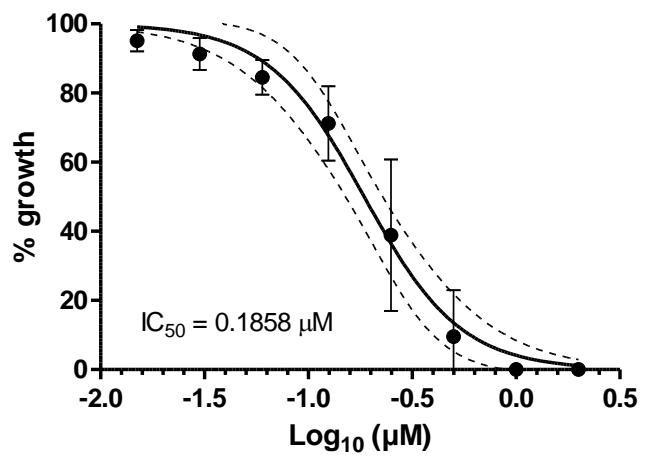

c. 1205 b. 1024

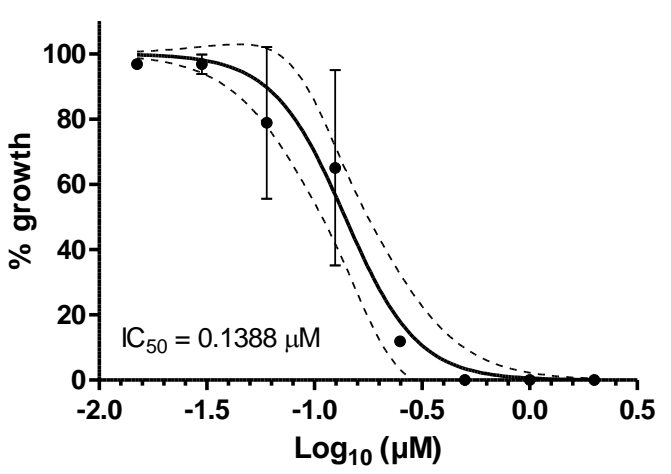

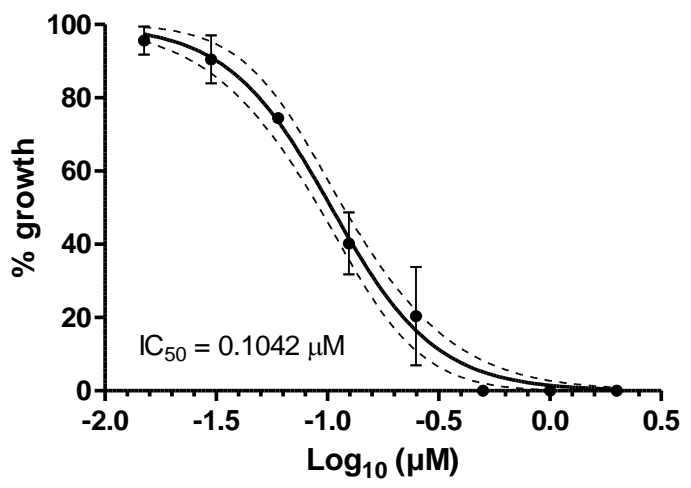

Figure 4.2 Dose-response curves of AgiM HiM3 antibacterial activity show low IC50 values

. The growth curves from Figure 1a-c were used to calculate the difference in the time to reach OD600 $=0.1$ compared to the untreated control. The results were plotted as a normalised response (\% growth) versus log concentrations. The IC50 values were determined using a nonlinear regression model with log (inhibitor) vs normalised response (variable slope). The dots represent the mean of three independent experiments with error bars representing SD, the solid lines show the fitted non-linear regression curve, and the dotted lines show the $95 \%$ confidence interval of the non-linear regression curve. 


\subsubsection{AgiM exhibit dose-dependent killing of A. baumannii}

To investigate the potential for AgiMs to be used against other Gram-negative bacteria, AgiM HiM3 was tested against Acinetobacter baumannii, a notorious nosocomial pathogen associated with high mortality rates and minimal therapeutic options. This opportunistic pathogen can survive for prolonged periods under a wide variety of environmental conditions; often populating medical equipment such as respirators and catheters as well as hospital tables and door handles leading to outbreaks of infection. These infections are difficult to treat, with MDR strains of $A$. baumannii acquiring resistance to almost all known antibiotics (70).

Standard growth assays were performed as described earlier, with A. baumannii isolates $\mathrm{AB} 6, \mathrm{AB} 10$ and $\mathrm{AB} 12$ and using a 2 -fold dilution series of AgiM, ranging from $0.015 \mu \mathrm{M}$ to $2 \mu \mathrm{M}$, and growth monitored for 20 -h at $37^{\circ} \mathrm{C}$.

It was found that the AgiM had a dose-dependent killing effect on A. baumannii strains, with increasing concentrations of AgiM HiM3 causing a proportional increase in the apparent lag time (Figure 4.3a-c). The treatment growth curves were right-shifted compared to the untreated control, and were approximately parallel, indicating a reduction in the inoculum. Clinical isolates AB6 and AB10 were similar, with no growth detected in concentrations $2 \mu \mathrm{M}$ and $1 \mu \mathrm{M}$; accordingly, the MIC was recorded as $1 \mu \mathrm{M}$. Clinical isolate $\mathrm{AB} 12$ was more sensitive to treatment with no growth detected at concentrations $2 \mu \mathrm{M}, 1 \mu \mathrm{M}, 0.5 \mu \mathrm{M}$ and $0.25 \mu \mathrm{M}$, as such the MIC was recorded as $0.25 \mu \mathrm{M}$. One-way ANOVA analysis of the area under the curve (AUC) for the isolate growth profiles showed all concentrations of AgiM HiM3 had a significant impact on the accumulated biomass $(\mathrm{p}<0.001)$ (Figure 4.3a-c). The antibacterial activity of AgiM HiM3 against $A$. baumannii was assessed by analysis of dose-response curves as described above for $P$. 
aeruginosa growth assays. The IC 50 values were $0.07476 \mu \mathrm{M}(95 \% \mathrm{CI}: 0.05885$ to 0.09833 $\mu \mathrm{M})$ in isolate $\mathrm{AB} 6,0.08658 \mu \mathrm{M}(95 \% \mathrm{CI}: 0.06520$ to $0.1212 \mu \mathrm{M})$ in isolate $\mathrm{AB} 10$ and $0.1152 \mu \mathrm{M}(95 \% \mathrm{CI}: 0.09302$ to $0.1426 \mu \mathrm{M})$ in isolate AB12 (figure 4.4a-c). These low values are consistent with strong antibacterial activity. 
a. AB6
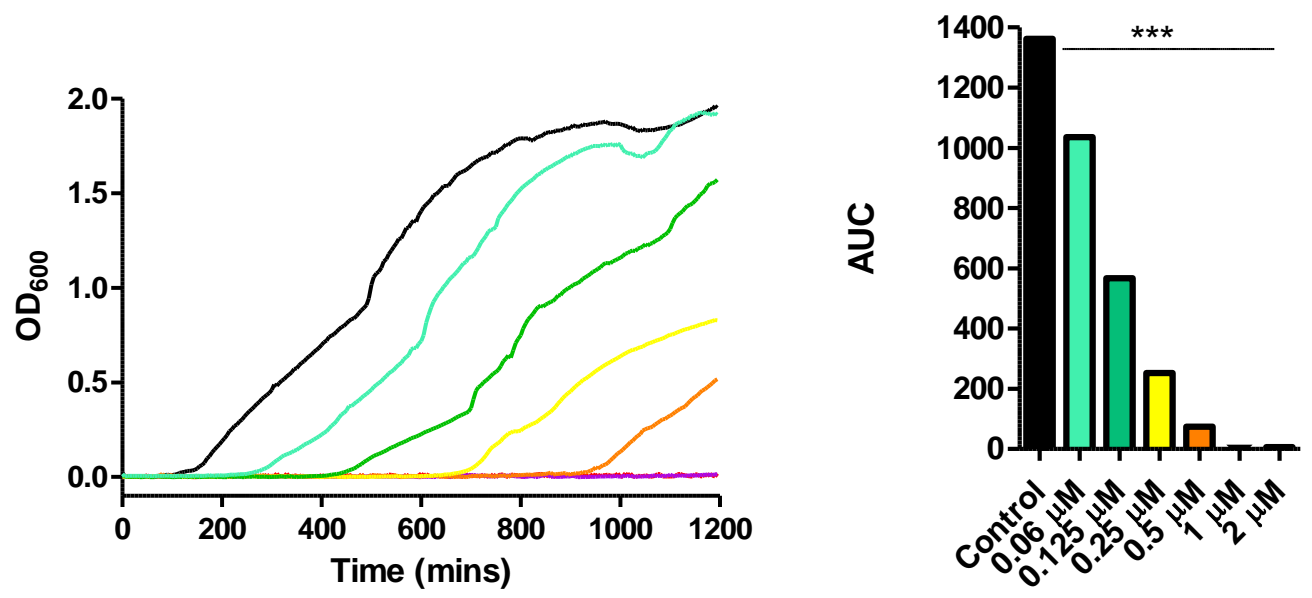

b. AB10
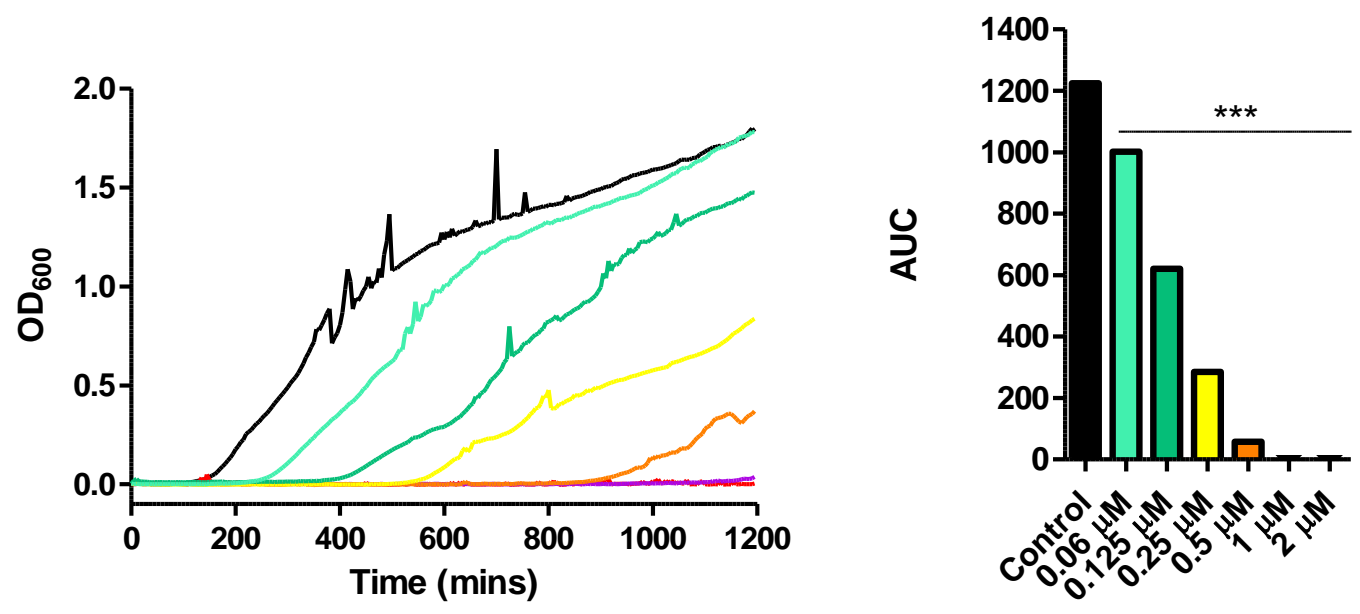

c. $\mathrm{AB12}$
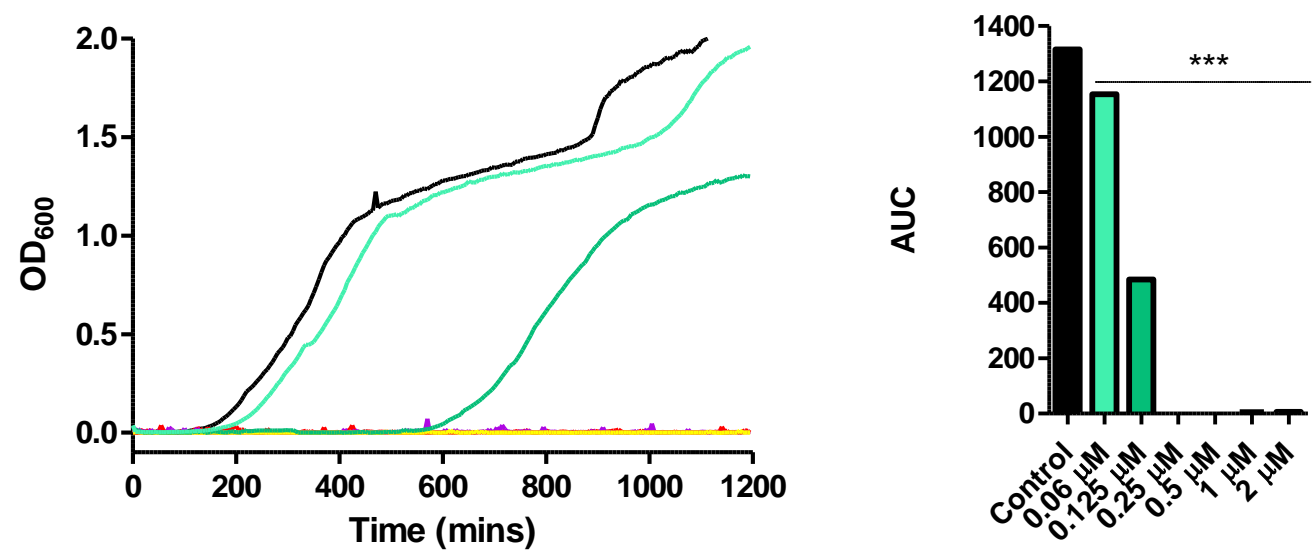

- Control $-2 \mu \mathrm{M}-1 \mu \mathrm{M}-0.5 \mu \mathrm{M}$
$-0.25 \mu \mathrm{M}-0.125 \mu \mathrm{M}-0.06 \mu \mathrm{M}$ 
Figure 4.3 Dose-dependent antibacterial activity of AgiM HiM3 against A. baumannii.

A. baumannii clinical isolates AB6, AB10 and AB12 were treated with a 2-fold dilution series of AgiM HiM3 at a concentration range of $2 \mu \mathrm{M}$ to $0.15 \mu \mathrm{M}$. Growth was monitored by turbidity at $600 \mathrm{~nm}$ every 5 minutes for 20 -h. The growth curves represent the mean of two independent experiments (left figures). The area under the curve (AUC) was calculated for each growth curve. The bar graphs represent the mean of two independent experiments, and the error bars show $\mathrm{SD}$ (right figures). P values were calculated using one-way ANOVA with Dunnet's post-hoc test to compare all means to the control. ${ }^{*} \mathrm{p}<0.05,{ }^{* *} \mathrm{p}<0.01, \mathrm{p}<0.001$ 
AB6

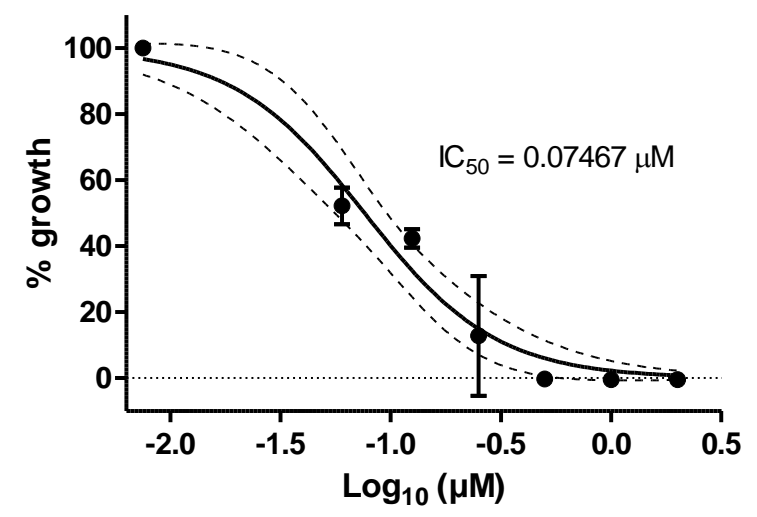

$\mathrm{AB} 10$

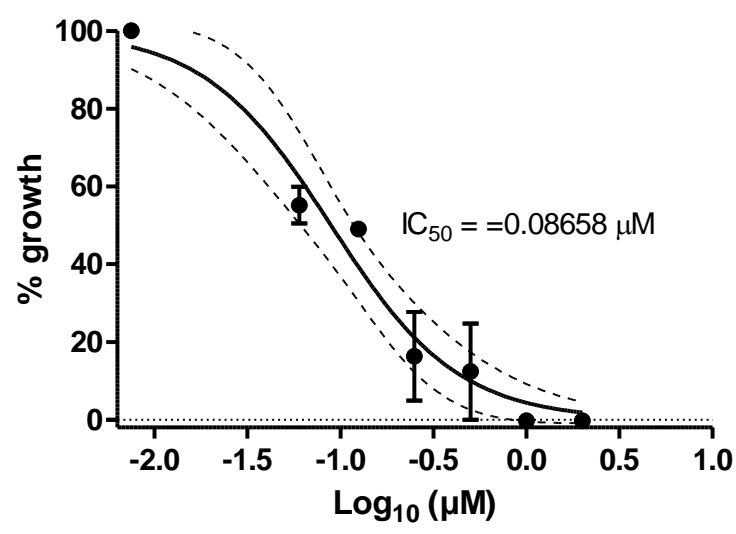

$\mathrm{AB} 12$

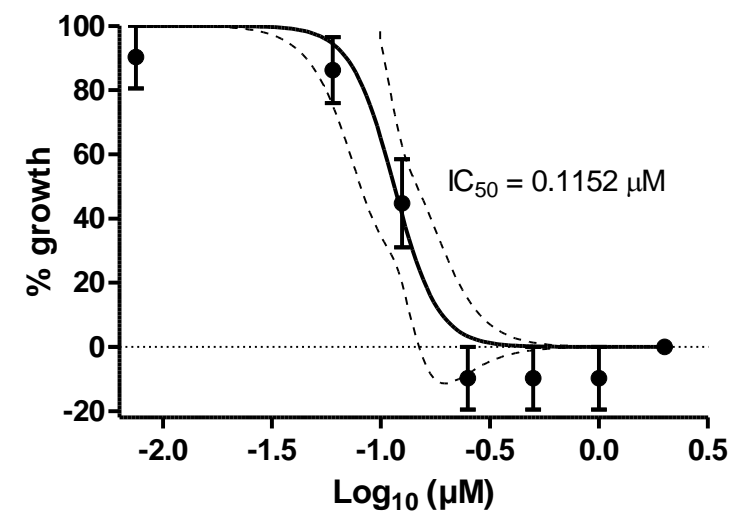

Figure 4.4 Dose-response curves of AgiM HiM3 antibacterial activity show low $\mathrm{IC}_{50}$ values against $\mathrm{A}$. baumannii.

The growth curves from Figure 3a-c were used to calculate the difference in the time to reach $\mathrm{OD}_{600}=0.1$ compared to the untreated control. The results were plotted as a normalised response (\% growth) versus log concentrations. The $\mathrm{IC}_{50}$ values were determined using a non-linear regression model with log (inhibitor) vs normalised response (variable slope). The dots represent 
the mean of two independent experiments with error bars representing SD, the solid lines show the fitted non-linear regression curve, and the dotted lines show the 95\% confidence interval of the non-linear regression curve. 


\subsubsection{AgiMs rapidly kill P. aeruginosa}

AgiM HiM3 displayed strong dose-dependent antibacterial activity, to further clarify the AgiMs mode of action time-kill kinetic studies were conducted. The time-kill assay provides a preliminary pharmacodynamic assessment, determining if a compound displays bacteriostatic or bactericidal activity. Bacteriostatic activity is defined as the decline or inhibition of growth of bacteria, without necessarily killing the inoculum; with bactericidal activity is defined as the rapid killing of the bacteria, demonstrated by a $>3$ log-fold decrease in colony-forming units (CFU), which confers $99.9 \%$ killing of the inoculum. The time-kill assays were performed in exponential phase inoculums of $P$. aeruginosa strain PA01 and clinical isolates 1079, 1205 and 1024 treated with AgiM HiM3 at concentrations $1 \times$ MIC and 4 x MIC, with samples taken at 15 mins, 30 mins, 1h, 2-h, 3-h, 4-h and 6-h time points, with a 0 min no treatment control. The effect of treatment was determined by enumeration of viable cells (CFU) at each time point, compared to the starting inoculum CFU and the percentage survival calculated.

Figure 4.5a-d shows the direct CFU counts of each time point at MIC (light blue) and $4 \mathrm{x}$ MIC (dark blue). Bactericidal activity was observed in two strains PA01 and 1024, with AgiM HiM3 causing a $3-\log 10$ reduction in CFU over the 6-h time period (Figure 4.5a-d). Comparatively, strains 1205 and 1079 only had a $1.6-\log ^{10}$ and $1.9-\log ^{10}$ reduction in CFU after AgiM treatment, respectively (Figure 4.5a-d). These data show AgiM exhibits bactericidal activity in P. aeruginosa, in a strain-specific manner.

Treatment of PAO1 at MIC resulted in a reduction of survival, with significant inhibition at $4 \mathrm{~h}(\mathrm{p}<0.05)$ but no bactericidal phase was observed (Figure 4.5a). PA01 treated with $4 \mathrm{x}$ MIC HiM3 caused a reduction in survival, with significance achieved at 3-h after treatment $(\mathrm{p}<0.05)$. Sterilisation of the inoculum was seen by 5 -h post-treatment with 4 
$\mathrm{x}$ MIC HiM3 and bactericidal activity confirmed by a $3-\log ^{10}$ reduction in growth compared to the control $(\mathrm{p}<0.001)$. These data show HiM3 at concentrations above MIC are necessary for bactericidal killing of PA01 strain of P. aeruginosa. The pattern of killing seen in PA01 shows rapid killing at the 30 min post-treatment period, although not significant. This could be due to the high level of SD across the four independent experiments of PA01.

Treatment of clinical isolates 1079, 1205 and 1024 at MIC resulted in significant growth inhibition compared to the control inoculum at 30 mins for 1079, and 2-h for strains 1205 and $1024(\mathrm{P}<0.05)$ (Figure 4.5a-d). Strain 1024 was the only clinical isolate to demonstrate bactericidal activity with a $3-\log 10$ reduction in growth compared to the control at 6-h post-treatment $(\mathrm{p}<0.001)$ (Figure $4.5 \mathrm{~d})$. Treatment of AgiM at $4 \times$ MIC significantly reduced survival from approximately $85-95 \%$ at 15 mins to $75 \%$ for strains 1079 and 1205, and 38\% for strain 1024 at 6-h post-treatment. AgiM Treatment of clinical isolates demonstrated rapid killing with significance at 30-min post-treatment of $4 \times$ MIC $(p<0.05)$ and continued significant inhibition over the 6 -h duration of the experiment $(\mathrm{P}<0.001)$.

These data demonstrate that clinical isolates 1079, 1024 and 1205 require higher treatment concentrations compared to the laboratory strain of P. aeruginosa. Conversely, AgiM treatment of the clinical isolates demonstrated the same trend as the laboratory strain with MIC treatment resulting in growth inhibition compared to the control, and 4 $\mathrm{x}$ MIC treatment causing improved efficacy with the potential of bactericidal behaviour. 
A: PA01

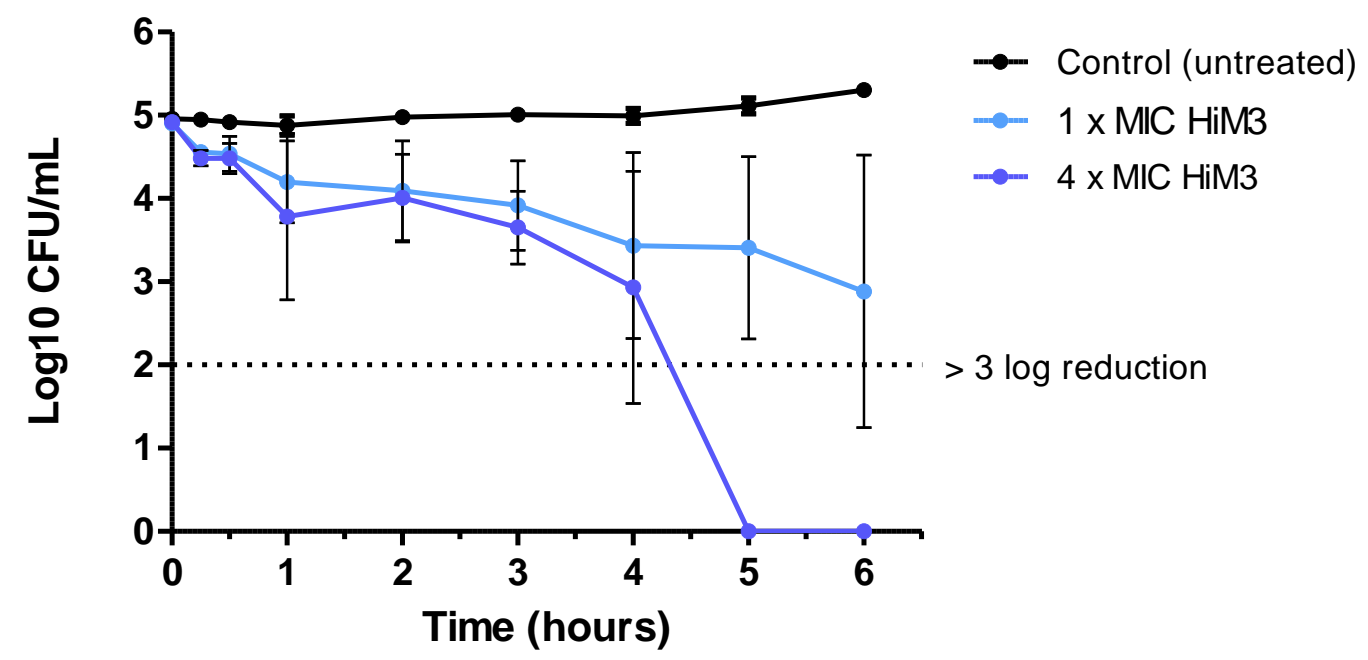

B: 1079

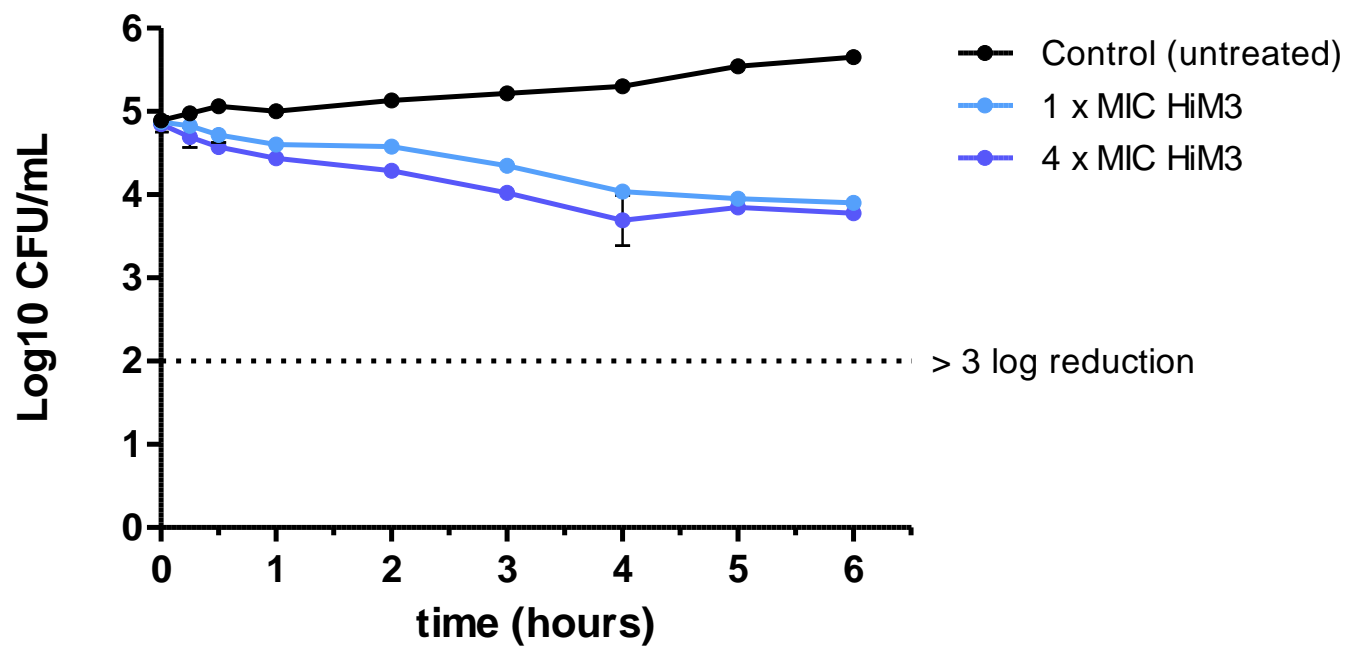




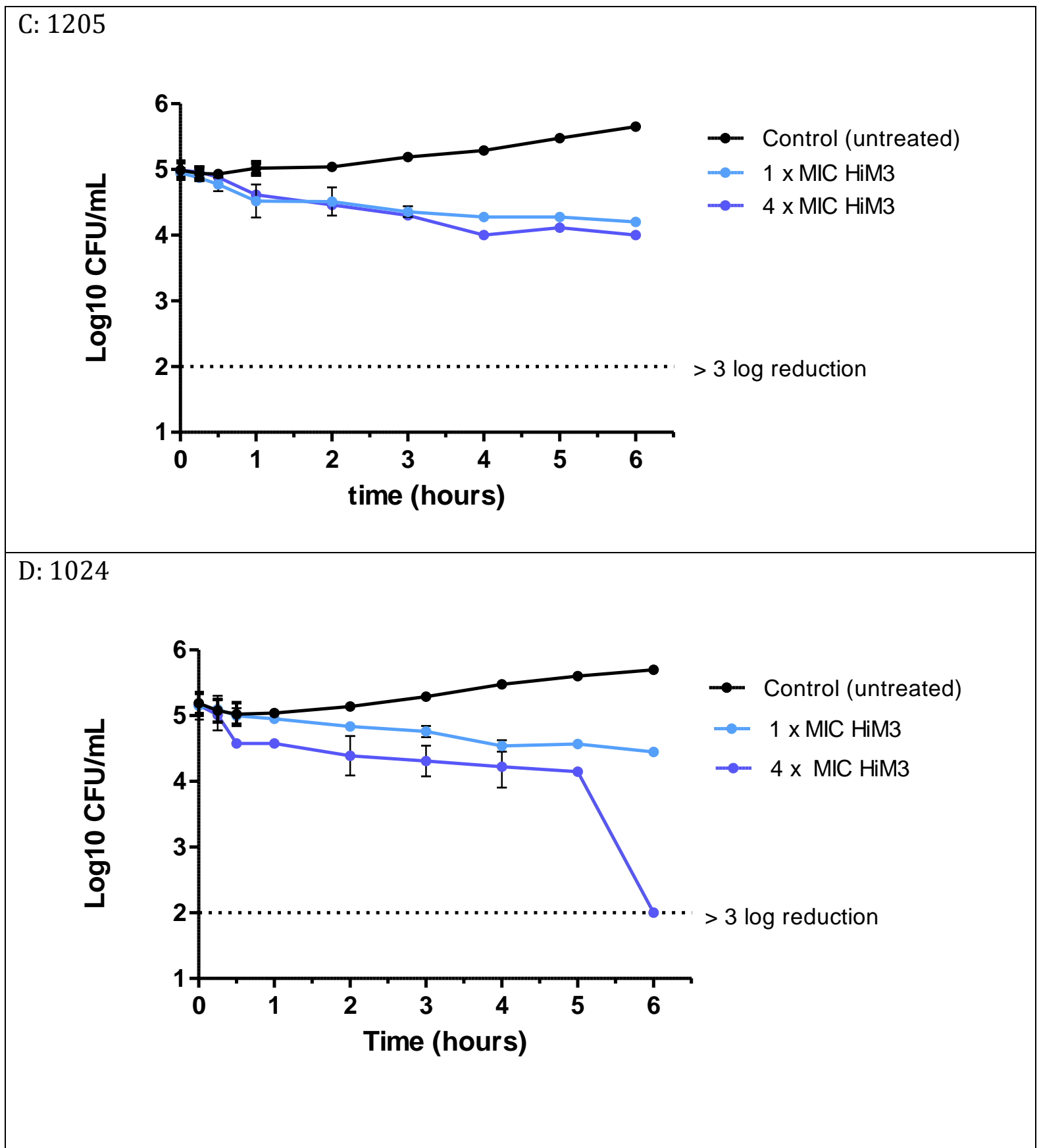

\section{Figure 4.5 Time-kill curves demonstrate AgiM killing of inoculum.}

The effects of treating exponentially growing P. aeruginosa strains PA01 (A), 1079 (B), 1205 (C) and 1024 (D) with MIC and 4xMIC AgiM Half iMotif 3 at various time points over 6 hours was determined by enumeration of viable cells (CFU count). The results are displayed as a direct CFU count The graphs represent four independent experiments for strain PAO1 and three independent experiments for other strains, with error bars representing SD. 
Figure 4.6 shows the fraction of surviving cells relative to the untreated control $(100 \%$ survival) for AgiM at concentration 4 x MIC at select time points (15 mins, 2-h, 4-h and 6h) in all strains of $P$. aeruginosa tested. The killing at 2-h post-treatment was significantly different $(\mathrm{p}<0.05)$ than the untreated control, with approximately $85-90 \%$ survival across all strains (Figure 4.5). The killing at 4-h post-treatment resulted in a significant reduction of survival to approximately $70-80 \%(\mathrm{p}<0.001)$ (Figure 4.6). At 6-h posttreatment, the strain PA01 had 0\% survival, with the clinical isolates decreasing to approximately 35 - 75\% survival (Figure 4.6).

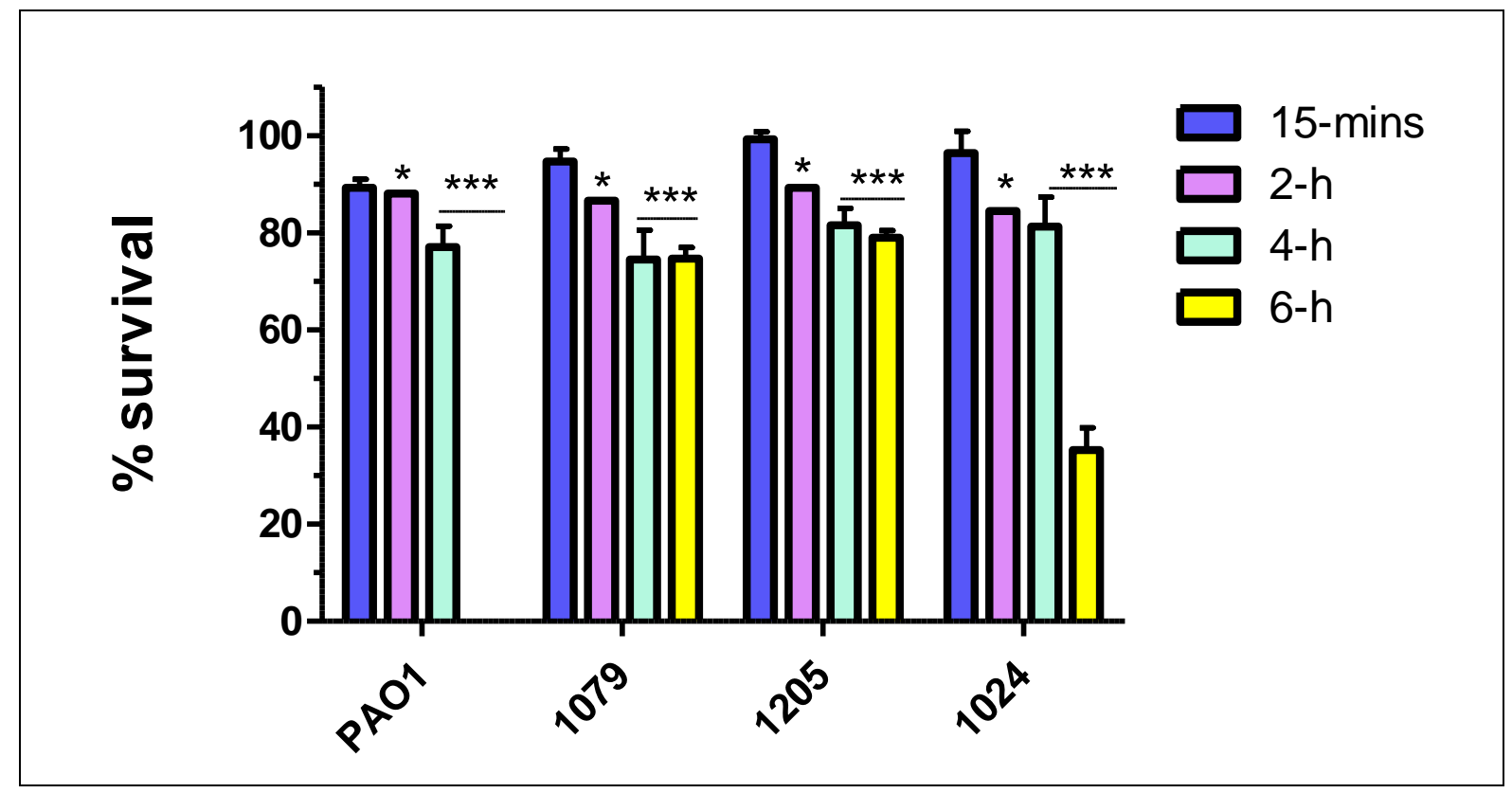

Figure 4.6 AgiMs rapidly kill P. aeruginosa strains.

The effects of 4 x MIC on all strains PA01, 1079, 1205 and 1024 are displayed as \% survival compared to the starting inoculum. The bar graph represents two independent experiments, with error bars representing SD. P values were calculated using a two-way ANOVA with Dunnet's post hoc test to compare all means to the $100 \%$ survival untreated control. ${ }^{*} p<0.05,{ }^{* * *} p<0.001$. 


\subsubsection{AgiMs inhibit the production of pyoverdine}

Pyoverdine is a siderophore produced by P. aeruginosa in iron-limited environments to sequester iron and transport it to the bacteria. It is implicated in the regulation and production of virulence factors and plays an essential role in the virulence of infections. Targeting virulence factors such as pyoverdine could aim to neutralise the activity, reducing the virulence of the bacteria. There is precedence for this, with molecules that interrupt the iron-acquisition system by competitively chelating iron have previously been reported to be effective antibacterials (25).

The aim of this experiment was to determine the effect of AgiMs on pyoverdine production in $P$. aeruginosa strains. Growth assays were prepared as described previously, with exponential phase inoculums treated with $0.25 \times$ MIC and $0.5 \times$ MIC AgiM HiM3, and growth and fluorescence measured every 5 mins for 20 -h at $37{ }^{\circ} \mathrm{C}$. The rationale of using sub-MIC concentrations of AgiM for this experiment was to elucidate pyoverdine perturbation in growing cultures of $P$. aeruginosa.

Figure 4.7 shows the growth $\left(\mathrm{OD}_{600}\right)$ and fluorescence (RFU) of each strain after treatment with AgiM HiM3 over 20-h. The untreated control of PAO1 reached an optical density (OD) of 1 at 625 mins, with pyoverdines characteristic fluorescence (Em. $405 \mathrm{~nm}$; Ex. $460 \mathrm{~nm}$ ) first detectable at 550 mins and peaking in fluorescence around 650 mins. Treatment with $0.25 \times$ MIC delayed bacterial growth reaching an OD = 1 until 850 mins while $0.5 \times$ MIC delayed $\mathrm{OD}_{600}=1$ until 1000 mins with both treatments successfully quenching pyoverdine fluorescence. The difference in growth inhibition of treatment groups compared to the control was statistically significant $(\mathrm{p}=0.003)$, with the inhibition of pyoverdine fluorescence in treatment groups compared to the control also statistically significant $(\mathrm{p}<0.001)$ (Figure 4.7). 
The clinical isolates 1205 and 1024 showed a similar trend to the laboratory strain PA01. The untreated control of 1205 reached $\mathrm{OD}_{600}=1$ at 650 mins, with pyoverdine fluorescence detectable at the same time point. Treatments 0.25 x MIC and 0.5 x MIC delay the $\mathrm{OD}_{600}=1$ to 850 mins and 1000 mins respectively, with no pyoverdine detectable for the duration of the experiment. The clinical isolate 1079 behaved differently. The untreated control reached an $\mathrm{OD}_{600}$ of 1 at 550 mins with pyoverdine fluorescence visible at the same time. Treatments of $0.25 \times$ MIC and $0.5 \times$ MIC reached an $0_{600}$ of 1 at 800 mins and 1000 mins respectively with corresponding pyoverdine fluorescence seen at these time points. This isolate is a strong producer of pyoverdine, with pyoverdine production unperturbed in the presence of sub-MIC concentrations of AgiM HiM3.

Overall these findings are consistent with AgiM HiM3 treatment reducing a proportion of the inoculum in a dose-dependent manner and delaying or inhibiting the production of pyoverdine in a strain-specific manner. 
PA01 a

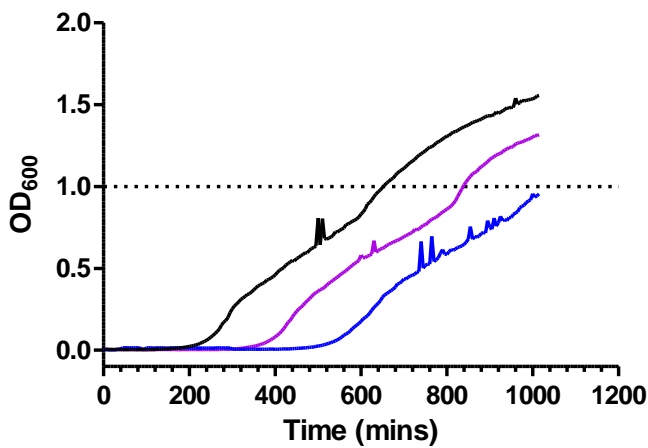

— untreated control (PAO1) $-0.5 \times \mathrm{MIC}-0.25 \times \mathrm{MIC}$ b

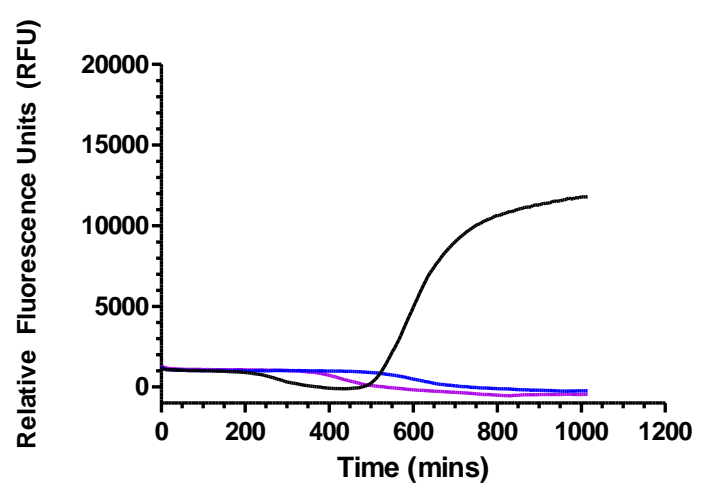

d

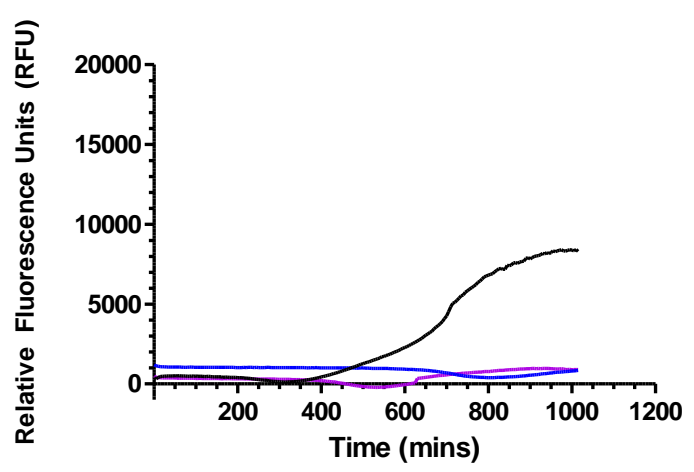




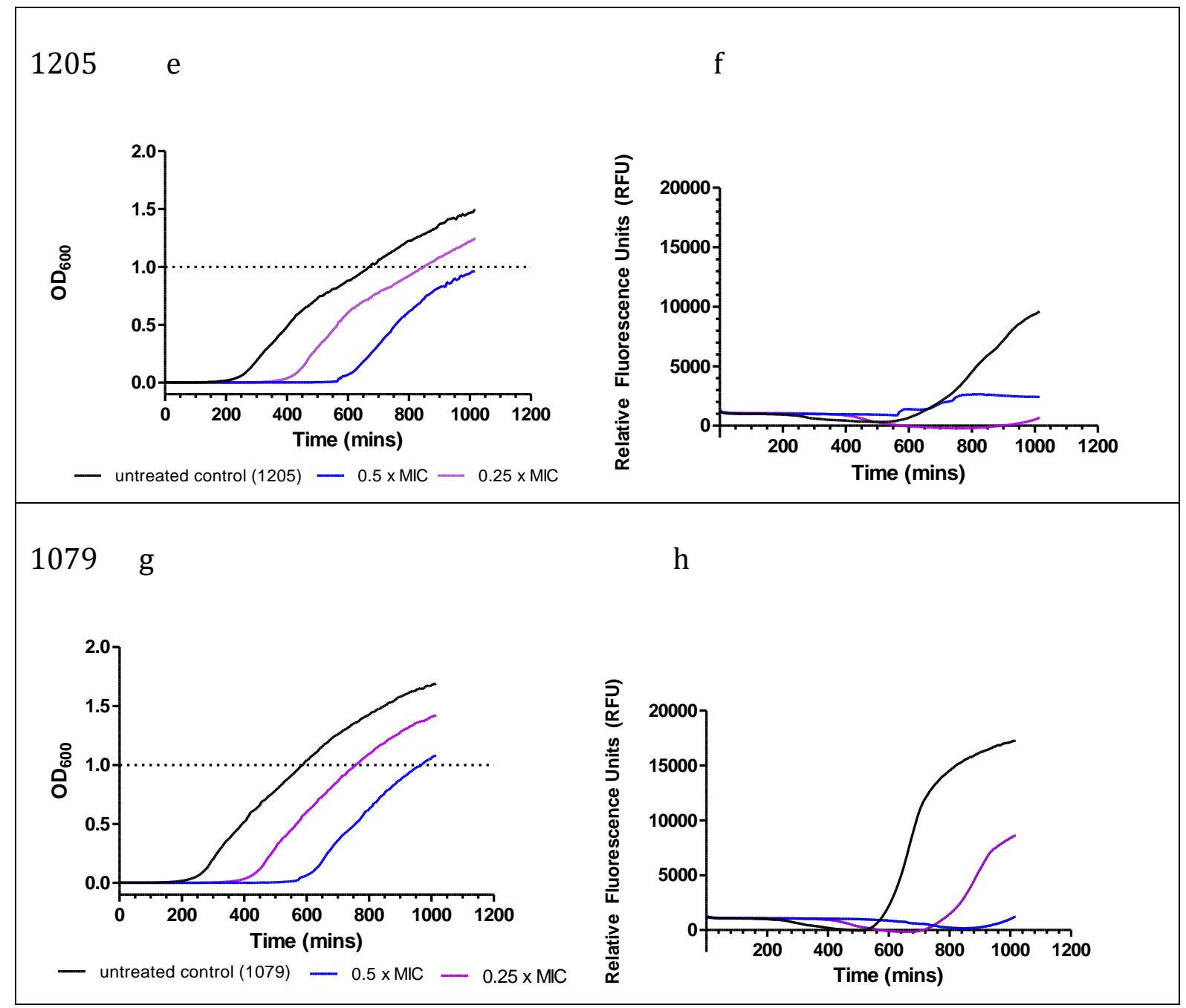

Figure 4.7 Subinhibitory concentrations of AgiM reduce siderophore pyoverdine.

4.7a-g. Pyoverdine production in P. aeruginosa strains PAO1 (A), 1024 (B), 1205 (C) and 1079 (D) was determined. Exponential phase cultures were treated with $1 / 4$ MIC or $1 / 2$ MIC AgiM HiM3, then incubated inside a Enspire 2300 Multilabel plate reader (Perkin Elmer, USA) under constant temperature control at $37^{\circ}$ C. Pyoverdine fluorescence (Ex. 405, Em. 455) and bacterial density (absorbance at $600 \mathrm{~nm}$ ) measurements were made every 5 minutes for 20 hours. 


\subsubsection{AgiMs are not substrates for efflux pumps}

To clarify the role of efflux pumps in the susceptibility of P.aeruginosa to AgiMs an experiment was designed to compare the sensitivity of the laboratory strain PA01 and efflux pump knockout mutant PA- $\Delta$ mexB. There are four major RND efflux pumps described in P. aeruginosa, with only MexAB-OprM expressed constitutively in wild-type P. aeruginosa. The knockout strain PA- $\Delta \operatorname{mex} B$, with an inactivated mexB gene has lost the function of proton motive force-driven efflux of antibiotics across the cytoplasmic membrane (10) effectively inactivating the MexAB-OprM efflux pump system.

To determine if AgiMs were substrates for P. aeruginosa efflux pumps, the laboratory strain PAO1 and an isogenic efflux pump mexB knockout mutant PA- $\Delta m e x B$ were investigated for their sensitivity to AgiM HiM3 and antibiotic ciprofloxacin. Exponential phase inoculums of PA01 and PA- $\triangle \operatorname{mexB}$ were treated with a 2-fold dilution series of AgiM and ciprofloxacin, and growth monitored for 18 -h at $37{ }^{\circ} \mathrm{C}$. The final $\mathrm{OD}_{600}$ measurements of treated wells were compared to the untreated control and used to calculate the percentage growth, which was plotted against the log concentration. The IC50 was determined using a non-linear regression model for log (inhibitor) vs normalised response (variable slope) (Figure 4.8a-b).

Strain PA- $\Delta m e x B$ as compared to PAO1 showed a significant increase in sensitivity to ciprofloxacin, with an $\mathrm{IC}_{50}$ of $<0.01 \mu \mathrm{g} / \mathrm{mL}$ compared to $0.1066 \mu \mathrm{g} / \mathrm{mL}$, respectively (Figure 4.8a). The difference in sensitivity is consistent with the efflux pump mexB knockout strain (PA- $\Delta$ mexB) losing the ability to extrude the antibiotic, making the bacteria more susceptible to ciprofloxacin, which is a known substrate of the MexABoprM efflux system (11). Comparatively, both PA- $\Delta$ mexB and PA01 had similar sensitivity to AgiM, with an IC50 of $0.1259 \mu \mathrm{M}$ and $0.1299 \mu \mathrm{M}$, respectively (Figure 4.8b). These data 
demonstrate that the presence or exclusion of the MexAB-oprmM efflux system does not affect AgiM efficacy.

Together, these data indicate AgiMs are poor efflux pump substrates, protecting the treatment from active extrusion from bacterial cells and allowing lower concentrations of AgiM to be effective. This also suggests $P$. aeruginosa may not be able to develop resistance to AgiMs through the overexpression of efflux pumps, which are a common resistance mechanism of MDR strains. 
A. Ciprofloxacin

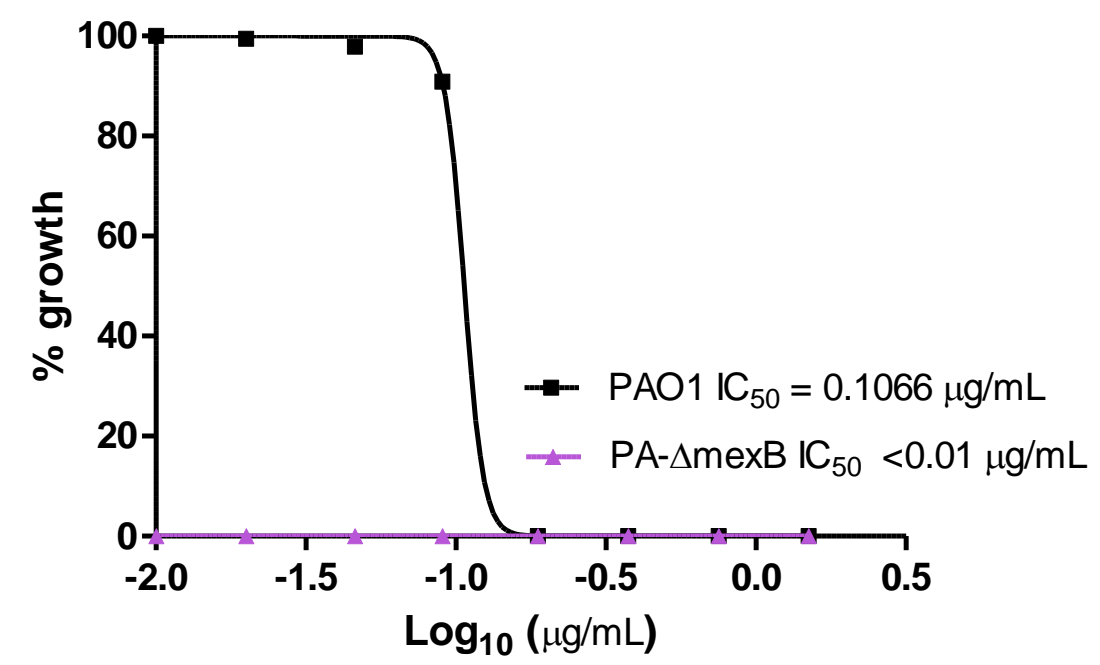

B. AgiM HiM3

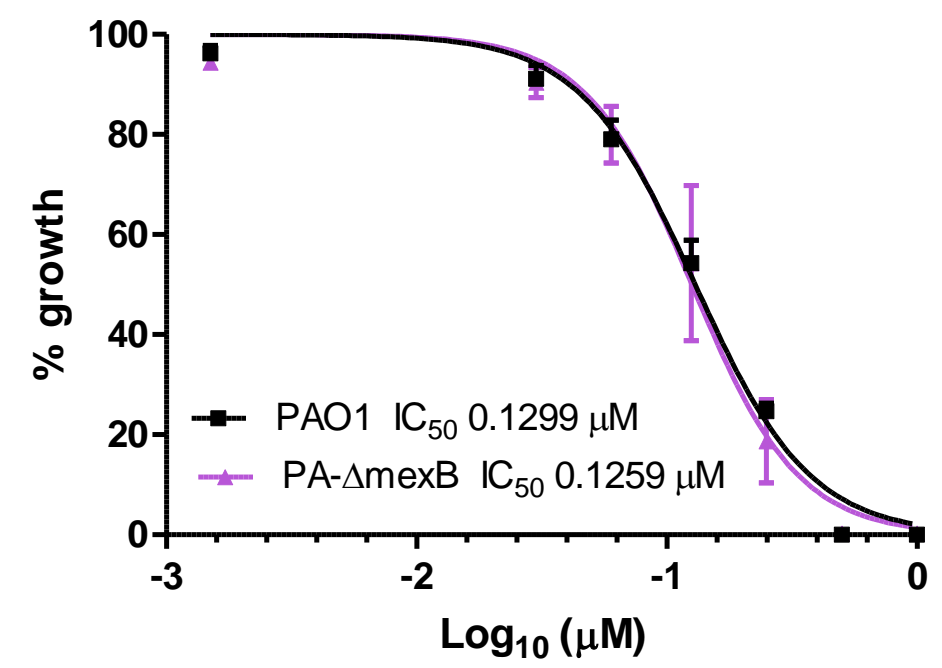

Figure 4.8 AgiM are not substrates for $P$. aeruginosa efflux pumps.

Exponentially growing cultures of strains PAO1 and PA- $\Delta$ mexB were treated with a 2 -fold dilution series of AgiM (ranging from $0.0015 \mu \mathrm{M}$ to $1 \mu \mathrm{M}$ ) or ciprofloxacin (ranging from 0.01 $\mu \mathrm{g} / \mathrm{mL}$ to $1.5 \mu \mathrm{g} / \mathrm{mL}$ ), with growth measured by $\mathrm{OD}_{600} \mathrm{~nm}$ over 18 -h at $37{ }^{\circ} \mathrm{C}$. The final $0 \mathrm{D} 600$ measurements were compared to the control and used to plot the percentage growth vs log concentration. The $\mathrm{IC}_{50}$ values were determined by non-linear regression for $\log$ (inhibitor) vs normalised response (variable slope) to compare the antibacterial effect of ciprofloxacin (A) and AgiM (B) in strains PA01 (black square) and PA- $\Delta$ mexb (purple triangle). The graphs represent the mean of two independent experiments and error bars show SD. 


\subsection{Key Findings}

The results from this chapter demonstrated the antibacterial activity of AgiM HiM3, with concentration-dependent killing observed in both P. aeruginosa and A. baumannii strains. The MIC across both bacteria ranged from $0.25 \mu \mathrm{M}$ to $1 \mu \mathrm{M}$, with low $\mathrm{IC}_{50}$ values indicating strong antibacterial activity in Gram-negative bacteria (Table 4-3).

Table 4-3 Summary of AgiM HiM3 activity against Gram-negative bacteria P. aeruginosa and $A$. baumannii

\begin{tabular}{lcc}
\hline $\begin{array}{c}\text { Pseudomonas } \\
\text { aeruginosa }\end{array}$ & MIC & IC $_{\mathbf{5 0}}(\mathbf{9 5 \%} \mathbf{C I})$ \\
\hline PA01 & & \\
\hline Clinical isolate 1079 & $0.5 \mu \mathrm{M}$ & $0.1378 \mu \mathrm{M}(0.1198$ to 0.1586$)$ \\
\hline Clinical isolate 1205 & $1 \mu \mathrm{M}$ & $0.1858 \mu \mathrm{M}(0.1522$ to 0.2268$)$ \\
\hline Clinical isolate 1024 & $1 \mu \mathrm{M}$ & $0.1042 \mu \mathrm{M}(0.09199$ to 0.1181$)$ \\
\hline \multicolumn{1}{c}{ Acinetobacter } & $1 \mu \mathrm{M}$ & $0.1388 \mu \mathrm{M}(0.11105$ to 0.1743$)$ \\
\multicolumn{1}{c}{ baumannii } & MIC & IC \\
\hline Clinical $(\mathbf{9 5 \%} \mathbf{C I})$ \\
\hline Clinical isolate AB6 & & \\
\hline Clinical isolate AB10 & $1 \mu \mathrm{M} 12$ & $0.07476 \mu \mathrm{M}(0.05885$ to $0.09833 \mu \mathrm{M})$ \\
\hline
\end{tabular}

Time-kill kinetic studies of the survival of P. aeruginosa treated with AgiMs provided preliminary pharmacokinetic information. AgiM demonstrated rapid killing of the inoculum, with bactericidal activity observed in $50 \%$ of the strains tested. The pattern of action observed over the 6-h period demonstrated AgiMs rapidly kill P. aeruginosa, with this killing effect increasing over the duration of the experiment. Treatment at MIC resulted in a significant reduction in survival across all strains of $P$. aeruginosa tested $(\mathrm{p}<0.001$ at 6 -h post-treatment). Comparison of treatment concentrations MIC and $4 \mathrm{x}$ MIC across the strains show both significantly inhibit growth at a similar rate, however increasing the AgiM concentration from $1 \times$ MIC to $4 \times$ MIC resulted in a change from growth-inhibiting behaviour to bactericidal behaviour in strains PA01 and 1024. The differences observed in treatment outcomes between P. aeruginosa strains PA01 and 
clinical strains could be due to the high SD seen over the four PA01 experiments. However, it is more likely due to the intrinsic traits of individual bacterial strains. It is possible the clinical isolates could be mucoid strains of $P$. aeruginosa which are known to have different antimicrobial susceptibilities than non-mucoid pseudomonas aeruginosa isolates (71).

Treatment with low concentrations of AgiMs resulted in the inhibition of pyoverdine production, an iron-sequestering siderophore associated with virulence of $P$. aeruginosa infections. Interfering with bacterial iron homeostasis is a potential therapeutic target, which may inhibit P. aeruginosa virulence and is a growing field of research. Sub-MIC concentrations of AgiM do not inhibit the growth of the bacteria; however, this treatment results in inhibition of pyoverdine. This suggests low concentrations of AgiMs could be used in conjunction with antibiotics for the attenuation of virulence by inhibition of pyoverdine. There are reports of attenuation of virulence as a viable treatment option for chronic and highly resistant strains of P. aeruginosa.

The results of this study indicated AgiMs are not substrates for MexB efflux pumps which are produced by $P$. aeruginosa. The upregulation of RND MexAB efflux pumps is associated with the development of resistance in P. aeruginosa, due to the active efflux of antimicrobials out of the bacterial cell. AgiM are not a substrate for these efflux pumps, which indicates that lower concentrations can be used and reduces the chance of resistance developing. 


\subsection{Discussion}

The antibacterial activity of AgiMs was demonstrated to be concentration-dependent, with higher doses killing an increasing proportion of the inoculum without significantly decreasing the growth rate of the remaining bacteria. This was demonstrated by the parallel growth curves, which increased in apparent lag time as the concentration of AgiM increased. These findings are consistent with publications of the antibacterial activity of AgNCs against E. Coli, two of which showed growth curves indicating the same dosedependent bactericidal effects (68)(72). This trend was observed in both P. aeruginosa and A. baumannii in this chapter, indicating AgiMs exhibit strong antibacterial activity against WHOs top two priority pathogens, in critical need of new therapeutics.

There are two expected growth profiles from antibacterial treatment, (1) the treatment may cause rapid killing of a proportion of the bacterial cells, effectively reducing the inoculum size without changing the rate of growth, demonstrated by an increase in the apparent lag time, or (2) the treatment may cause a reduction in the growth rate without killing the bacteria. The results of this study indicate that the killing effect of AgiMs does not significantly impair the growth rate of the bacteria, due to the increase in apparent lag time observed. These findings suggest AgiMs rapidly kill a fraction of the inoculum, which then re-grows at the same rate as the untreated control over the 20-h duration of the experiment. The resumption of growth could be due to the level of $\mathrm{Ag}^{+}$in the AgiM complex dropped below a therapeutic threshold, or the stability of the AgiM was degraded in the presence of the growth medium LB below cytotoxic levels. The predicted mechanism of action for AgiMs is the toxic effect of $\mathrm{Ag}^{+}$binding along the helical chain of DNA, inhibiting DNA function and leading to cell death, as reported in the literature by

(Modak and Fox). The dose-dependent killing effect shows the higher concentrations of 
$\mathrm{Ag}^{+}$in these treatments take longer to fall below the therapeutic threshold, thus delaying the growth of the inoculum. The highest concentrations of AgiM used $2 \mu \mathrm{M}$ and $1 \mu \mathrm{M}$ resulted in the sterilisation of the inoculum

AgiM treatment resulted in rapid killing of the inoculum, demonstrated by time-to-kill assays with a significant reduction in surviving cells at 2-h post-treatment across all strains of $P$. aeruginosa. It is predicted that this rapid killing is facilitated by $\mathrm{Ag}^{+}$most likely decreasing membrane permeability and allowing the influx of toxic levels of $\mathrm{Ag}^{+}$ into the cell. This effect is well characterised in the literature, with $\mathrm{Ag}^{+}$interacting with thiol proteins in the bacterial cell membrane as well as enzymes of the electron transport chain causing protein inhibition and increased cell permeability (36)(73). Morphological changes to the bacterial cell membrane by $\mathrm{Ag}^{+}$has been reported by Jung and colleagues, who showed E. coli cells were cracked and ruptured 2-h after $\mathrm{Ag}^{+}$treatment using TEM analysis (49).

The antibacterial activity of AgiMs resulted in the inhibition of pyoverdine production in P. aeruginosa strains. The link between pyoverdine and virulence has been clearly established in many contexts, including in C. elegans and in murine infections. The link between pyoverdine and virulence observed in C. elegans showed the level of pyoverdine produced directly correlated with the severity of disease and survival rate (25)(74). It has also been demonstrated in human trials, with Kang et al. reporting a correlation with pyoverdine production and virulence in P. aeruginosa isolates from CF patients. They reported pyoverdine production in vitro and in vivo correlated, allowing in vitro measurements to serve as a method to predict virulence (25). Treatment of AgiM does not target pyoverdine production, but the demonstration that sub-MIC concentrations of AgiM inhibit the production of this virulence determining siderophore shows the 
potential clinical utility of AgiMs. One strategy to circumvent the issue of MDR infections which do not respond to the current antibiotics is the use of anti-virulent compounds to disrupt pathogenesis without directly compromising bacterial growth. There is precedence for this, with the targeting of pyoverdine through fluoropyrimidines to reduce host mortality has been successfully achieved and published (75). A potential clinical use for AgiMs could be the attenuation of pyoverdine production, which may help the host immune defences to mitigate pathogenesis and clear the infection.

AgiMs are not a substrate for $P$. aeruginosa efflux pumps. The upregulation of RND efflux pumps in P. aeruginosa is associated with the development of MDR strains, and could in part explain the chronic infections of this opportunistic pathogen. This is reported in the lungs of cystic fibrosis patients, which present a harsh environment that leads to genomic adaption. Commonly mutated genes in $P$. aeruginosa infections are the negative regulators of RND efflux systems, with mexZ (a negative regulator of MexXY-OprM) the most frequent (25). Avoiding efflux out of the bacterial cell allows low concentrations of AgiM to be effective, which is vital when developing silver-based antibacterials. Potentially allowing a therapeutic dose of AgiM to be clinically viable and avoid the cytotoxic side effects of high doses of silver as well as reduce the potential of resistance developing. 


\section{CHAPTER 5. ANTIBIOFILM ACTIVITY OF AGIMS}

\subsection{Aims and rationale}

The ability of $P$. aeruginosa to form biofilms reduces the efficacy of antimicrobial treatments contributing to the high incidence of resistant infections. These unique bacterial communities can form on indwelling devices such as ventilators, catheters and medical implants, and soft tissue such as diabetic ulcers and the lungs of cystic fibrosis patients (16)(76). These infections can endure aggressive antibiotic therapy, often resulting in the surgical removal of the colonised area (77). The resistance to antimicrobials can be attributed to a combination of factors such as reduced permeability due to the extracellular matrix, increased efflux pump activity to remove antimicrobials that enter the cell and expression of resistance genes (29). Biofilm age is also known to influence the level of antimicrobial resistance. Finding antimicrobials with antibiofilm abilities is of great importance to avoid the undesirable outcome of surgical intervention and mortality caused by these resistant infections.

Silvers ability to challenge and disrupt biofilms is well reported, demonstrated by the ability of silver nitrate $\left(\mathrm{AgNO}_{3}\right.$ ), silver sulfadiazine $(\mathrm{AgSD})$ and AgNPs to penetrate $P$. aeruginosa biofilms in vitro (78)(79). Therefore, it was hypothesised that AgiMs would exhibit antibiofilm activity with penetration of established biofilms by disruption of the extracellular matrix and killing of the cells within the biofilm. The antibiofilm activity of AgiM was assessed using three metrics, (1) the ability of the treatment to inhibit planktonic growth of cells shed by the biofilm and (2) the ability of the treatment to disrupt biofilm biomass assessed by crystal violet staining and (3) the ability of the 
treatment to reduce the number of viable cells remaining in the biofilm evaluated by enumeration of CFU.

The experiments in this chapter evaluated the antibiofilm activity of AgiMs against $P$. aeruginosa strains PA01 and isolates 1079, 1024 and 1205. Biofilms were grown on MBEC devices for 20-h or 44-h to observe the efficacy of AgiM treatment on biofilms at different stages of maturation, with 20 -h biofilms characterised as 'young' and 44-h biofilms characterised as 'mature'.

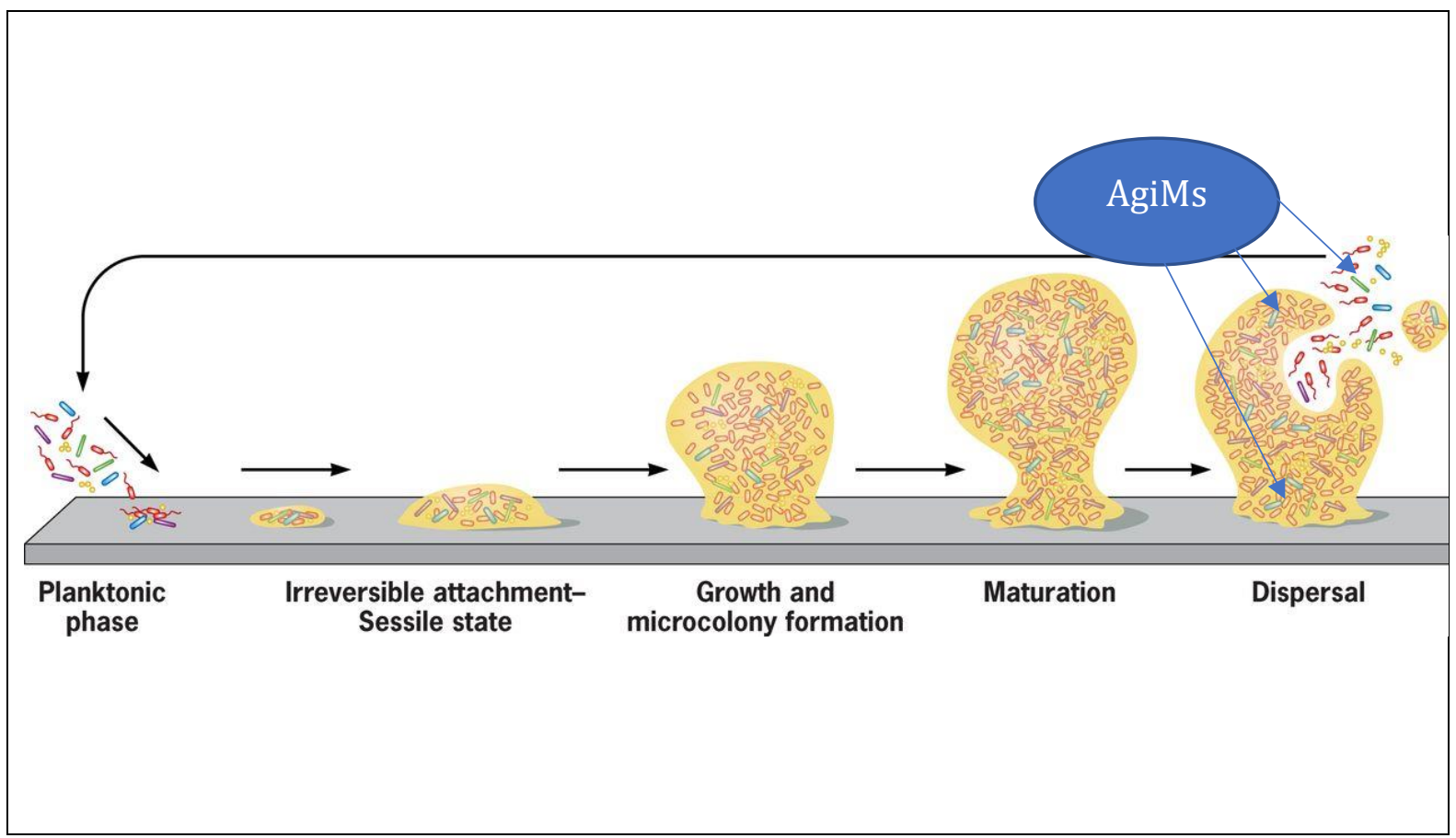

Figure 5.1 Proposed antibacterial activity of AgiMs on established biofilms.

This study aims to investigate the antibiofilm activity of AgiM on established biofilms. The lifecycle of biofilms is illustrated in the diagram above; the first step is the adherence of planktonic cells to biological or prosthetic surfaces, mediated by several factors including motility, extracellular adhesive appendages and secreted adhesins. As the cells colonise the surface, the second step of maturation begins, with a change in expression of bacterial genes involved in the establishment of the extracellular matrix which will encase the cells in a protective shield of exopolysaccharides. The third stage is dispersal, which can be activated by stress conditions or changes in nutrient availability. The major aims of this chapter are to establish if AgiM treatment (1) kills shed cells from the biofilm (2) decrease biomass of the biofilm, and (3) kills remaining cells left in the biofilm after treatment. Reprinted from Clinical Microbiology Reviews, Magana et al., Options and Limitations in Clinical Investigation of Bacterial Biofilms, Apr 4, 2018, with permission by American Society for Microbiology Copyright (C) 2018 


\subsection{Results}

\subsubsection{AgiMs reduce the viability of shed cells from biofilms}

Biofilms were grown on MBEC devices for 20-h or 44-h using the laboratory strain PAO1 and clinical isolates 1079, 1024 and 1205. The pegs of MBEC devices were immersed in different concentrations of AgiM treatment for 6-h, ranging from $0.6 \mu \mathrm{M}$ to $5 \mu \mathrm{M}(0.5 \mathrm{x}$ MIC to $5 \mathrm{x}$ MIC for clinical isolates). The expectation of using concentrations at or above the MIC was that any shed cells should not grow. At the completion of treatment, the peg lid was removed, and any growth in the treatment plate containing shed cells from the biofilms was measured $\left(\mathrm{OD}_{600}\right)$.

There was a reduction in growth for both 20 -h and $44-\mathrm{h}$ biofilms for all strains of $P$. aeruginosa treated with $5 \mu \mathrm{M}$ AgiM (5 x MIC)(Figure 5.2a-h). Shed cells from 20-h biofilms were reduced by $57 \%, 52 \%, 37 \%$ and $74 \%$ compared to the control for strains PA01, 1079,1024 and 1205 respectively (Figure 5.2a,c,e,g). The reduction of shed cells from 44h biofilms compared to the control was $63 \%, 73 \%, 42 \%$ and $38 \%$ for strains PA01, 1079, 1024 and 1079 respectively (Figure 5.2b,d,f,h). The inhibition of shed cells was significant for strains PA01, 1079 and 1205 (p=0.0001), but not for isolate 1024. This is most likely due to a combination of reduced susceptibility to AgiM treatment by isolate 1024 and increased variance in the measurements.

The concentration range of $0.5 \times$ MIC up to $5 \times$ MIC - the use of $5 \times$ MIC was needed to achieve a significant reduction in the growth of shed cells. This is consistent with the literature that indicates the sessile cells in biofilms are more resistant than planktonic cells, with reports of 10-1000 -fold increase in MIC to kill sessile bacteria compared to their planktonic counterparts. Concentrations significantly higher than the MIC - growth 
was still detected, although it was reduced. This decreased sensitivity to antimicrobial challenge is consistent with previously published reports.

Figure $5.3 \mathrm{a}$ and $5.3 \mathrm{~b}$ compare the ability of $5 \mu \mathrm{M}$ AgiM to inhibit new 20 -h biofilms and mature 44-h biofilms. These data confirm the findings that the $5 \times$ MIC AgiM reduces the growth of both 20 -h and 44-h biofilms in all strains except 1024. Figure 5.3c illustrates the variability of inhibition of shed cells relative to the control for 20 -h and 44 -h biofilms. These data show there is no significant difference in inhibition between 20 -h and $44-\mathrm{h}$ biofilms in strains PA01, 1024 and 1205. In comparison, strain 1079 shows a significant difference in inhibition with 44-h biofilms demonstrating greater susceptibility to AgiM treatment than the 20-h biofilm $(\mathrm{p}<0.01)$ (Figure 5.3c).

Overall, these data are consistent with high concentrations of AgiM (10 x MIC) reducing the viability of cells shed from 20-h and 44-h P. aeruginosa biofilms. 


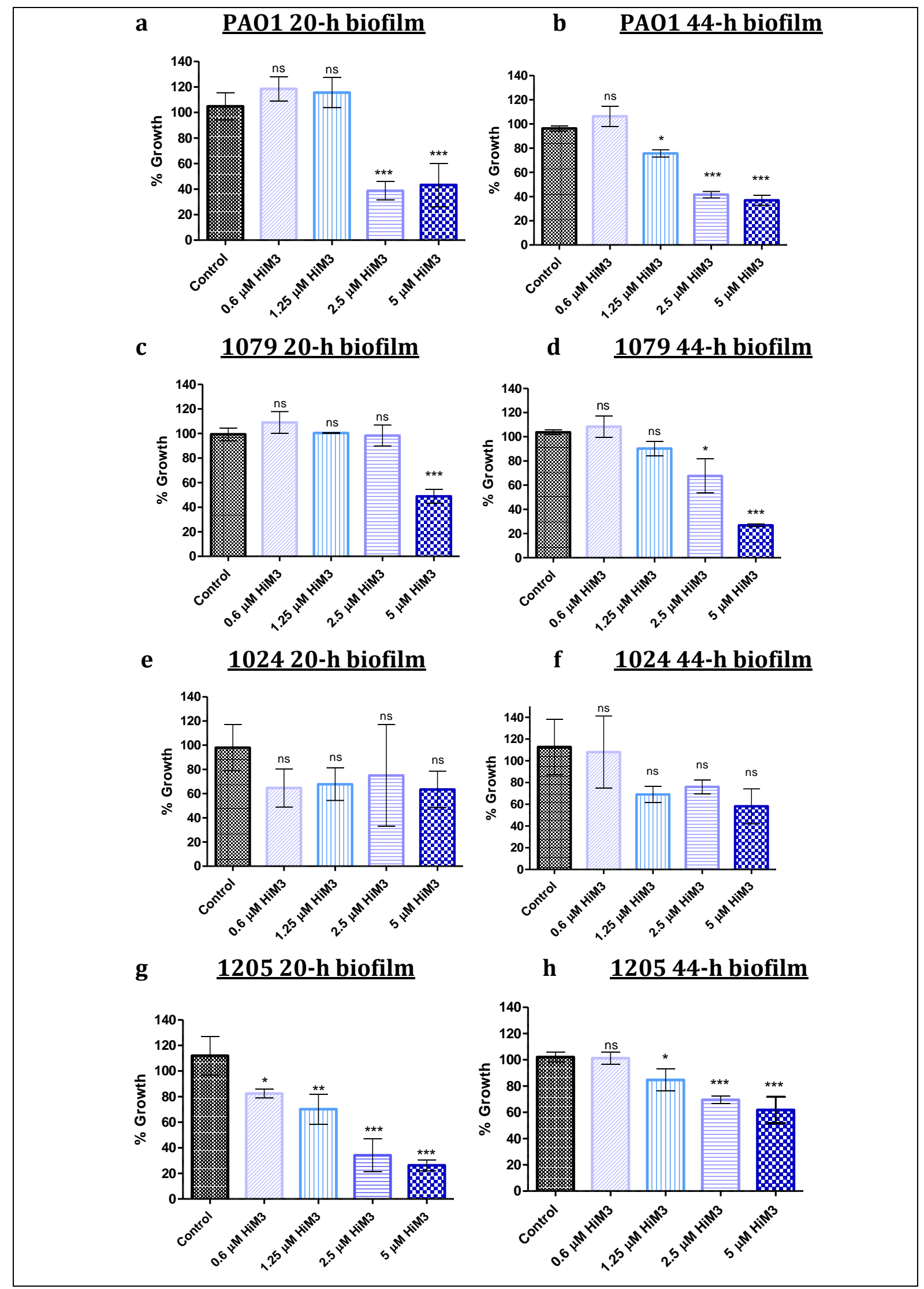


Figure 5.2 High concentrations of AgiM treatment reduce planktonic shedding from 20-h and 44-h biofilms.

5.2a-h: Biofilms were grown on MBEC devices for 20 -h or 44 -h at $37{ }^{\circ} \mathrm{C}$ with gentle agitation. Biofilms were grown from planktonic suspensions of P. aeruginosa strains PA01 (Figure 5.2a,b), clinical isolate 1079 (Figure 5.2c,d), clinical isolate 1079(Figure 5.2e,f), and clinical isolate 1205 (Figure 5.2g,h). Biofilms were then exposed to antimicrobial challenge for 6-h by treatment of AgiM at concentrations of $0.6 \mu \mathrm{M}, 1.25 \mu \mathrm{M}, 2.5 \mu \mathrm{M}$ and $5 \mu \mathrm{M}$. The MBEC devices were then removed, and the treatment plates were measured $\left(\mathrm{OD}_{600}\right)$ to evaluate the growth of cells shed from the biofilm. Bar charts represent the mean of three independent experiments with SD. The p-values were calculated using one-way ANOVA with Dunnets post hoc test to compare all treatments to the untreated control. $\left({ }^{* * *} \mathrm{p}=0.0001\right.$; $^{*} \mathrm{p}<0.05 ; \mathrm{ns}=$ no significance) 


\section{3a 20-h biofilm}

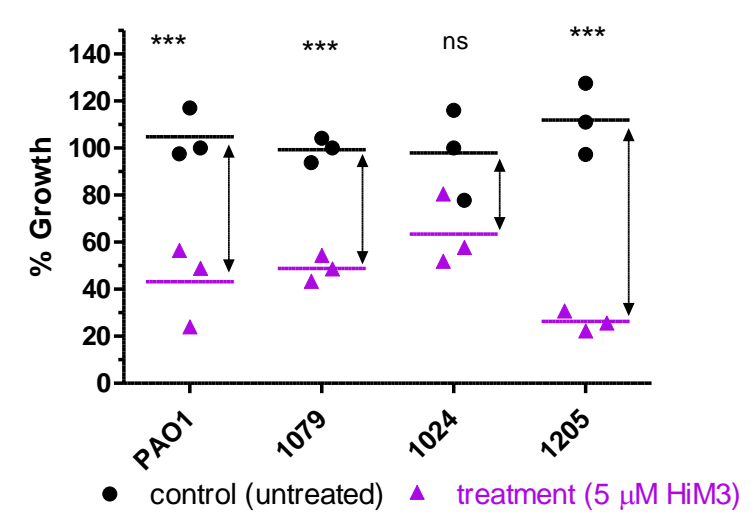

\section{3b 44-h biofilm}

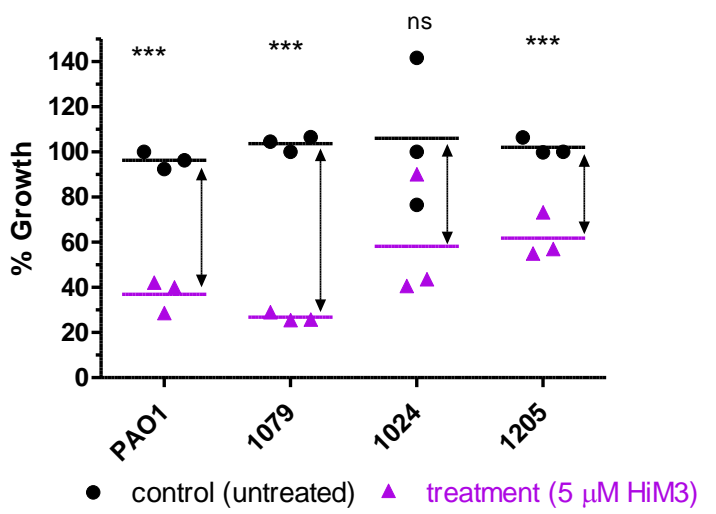

$5.3 c$

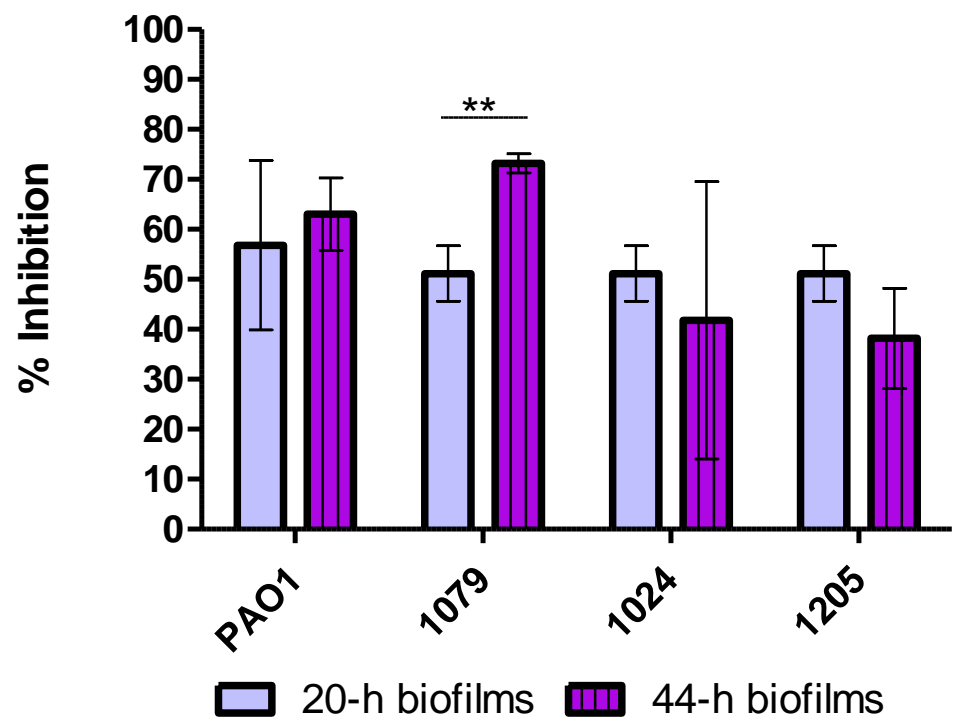

Figure 5.3 Comparison of highest concentration AgiM treatment (5 $\mu \mathrm{M})$ with untreated control on planktonic cells shed from $20-\mathrm{h}$ and $40-\mathrm{h}$ biofilms.

P. aeruginosa strains PA01, 1079, 1024 and 1205 were used to grow biofilms on MBEC devices for 20-h (Figure 2a) or 44-h (Figure 2b), followed by treatment of $5 \mu \mathrm{M}$ AgiM for 6-h. Figure 2c shows the comparison of the growth of shed cells for 20 -h and 44 -h biofilms of each strain tested. Figure $2 \mathrm{c}$ P values determined by unpaired t test. ${ }^{* *} \mathrm{p}=0.0029$ 


\subsubsection{AgiMs disrupt biofilm biomass}

To determine whether AgiM treatment disrupted biofilm mass, biofilms were grown for 20-h or 44-h and challenged with AgiM concentrations in a range of $0.6 \mu \mathrm{M}-5 \mu \mathrm{M}$ for 6h, with biomass quantified by crystal violet $(0.05 \%)$ staining. Figure 5.4 a-h shows the effect of AgiM treatment on biofilm biomass presented as the fraction of biomass remaining on the pegs after treatment compared to the untreated control.

Treatment of $20-\mathrm{h}$ biofilms at all AgiM concentrations caused a reduction in biomass compared to the control, with a statistically significant reduction in strains PA01, 1079 and 1205 at concentrations of $1.25 \mu \mathrm{M}, 2.5 \mu \mathrm{M}$ and $5 \mu \mathrm{M}$ (at least $\mathrm{p}<0.01$ ). These data indicate AgiM treatment is effective at biomass disruption in the younger 20 -h biofilms at concentrations as low as $1.25 \mu \mathrm{M}(1.25 \mathrm{x}$ MIC for clinical isolates and $2.5 \mathrm{x}$ MIC for PA01). Treatment of 44-h biofilms at all AgiM concentrations was less effective than in 20-h biofilms (Figure $5.4 \mathrm{a}$-h, Figure 5.5c). Only the highest concentrations of $5 \mu \mathrm{M}$ AgiM resulted in a significant reduction in biomass for two strains, 1079 and $1024(\mathrm{p}<0.0 .5)$. Treatment of 44-h biofilms with low concentrations of $0.6 \mu \mathrm{M}$ and $1.25 \mu \mathrm{M}$ was ineffective, causing a uniform increase in biomass compared to control for all strains tested; however, this was not statistically significant (Figure 5.4b,d,f,h). These results indicate AgiM is less effective in mature 44-h biofilms, which is likely due to the decreased permeability seen in more mature biofilms that have a fully established extracellular polysaccharide matrix compared to younger biofilms.

The highest concentration of $5 \mu \mathrm{M}$ AgiM ( $5 \times \mathrm{MIC}$ ) caused the most significant reduction in biomass compared to the control in both 20 -h and 44-h biofilms, with this effect always greater in the younger 20-h biofilms (Figure 5.5 a,b,c). This was clearly demonstrated in biofilms produced by PA01, with a 57\% reduction in 20 -h biofilm biomass compared to 
only $9 \%$ reduction in 44 -h biofilm biomass after treatment with $5 \mu \mathrm{M}$ AgiM $(\mathrm{p}=0.015)$ (Figure 5.5c).

High variance in strain susceptibility was observed across both 20 -h biofilms and 40-h biofilms (Figure $5.4 \mathrm{a}$-h). Strain 1205 was the most susceptible for 20-h biofilms, demonstrating $80 \%$ reduction in biomass after $5 \mu \mathrm{M}$ AgiM, but was significantly less susceptible for 44 -h biofilms with only $39 \%$ reduction in biomass. Strain 1024 was the most susceptible for 44-biofilms, with $5 \mu \mathrm{M}$ AgiM resulting in a 53\% reduction of biomass compared to the control. Differences in strain susceptibility are to be expected between the laboratory strain and clinical isolates, with this variation in effect increased by the high variance of measurements.

Overall, these data demonstrate treatment of AgiM reduces biofilm biomass, with 20-h biofilms more susceptible to AgiM disruption than mature 44-h biofilms. 


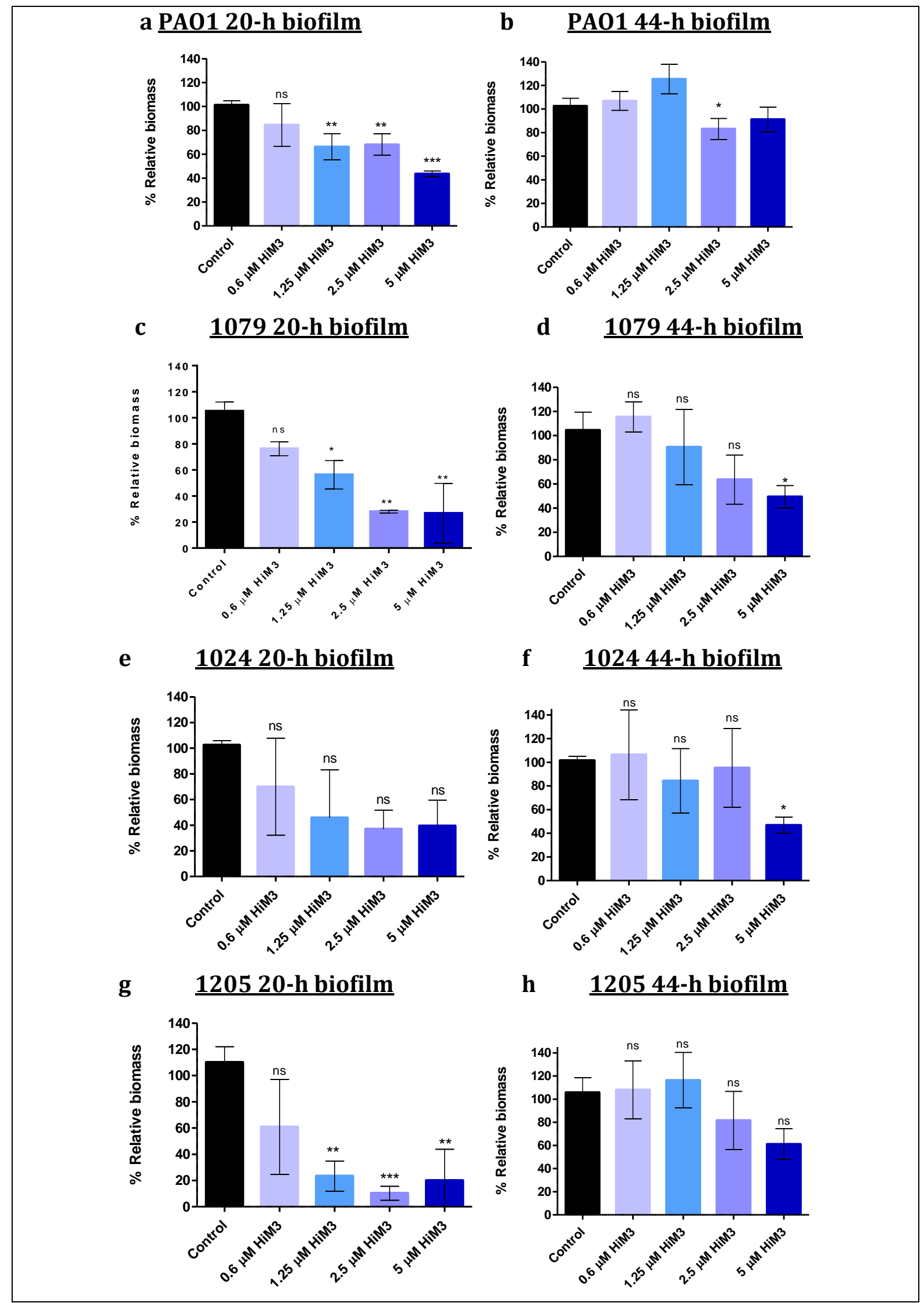

Figure 5.4 High concentrations of AgiM treatment disrupt 20-h and 44-h biofilms

High concentrations of AgiM treatment disrupt 20-h and 44-h biofilms. Biofilms were grown on MBEC devices for 20 -h or 44 -h at $37{ }^{\circ} \mathrm{C}$ with gentle agitation. Biofilms were grown from 
planktonic suspensions of $P$. aeruginosa strains PA01 (Figure 3a,b), clinical isolate 1079 (Figure $3 \mathrm{c}, \mathrm{d}$ ), clinical isolate 1079(Figure 3e,f), and clinical isolate 1205 (Figure 3g,h). Biofilms were then exposed to antimicrobial challenge for 6-h by treatment of AgiM at concentrations of $0.6 \mu \mathrm{M}, 1.25$ $\mu \mathrm{M}, 2.5 \mu \mathrm{M}$ and $5 \mu \mathrm{M}$. Antibiofilm activity assessed by the disruption of biofilm biomass measured by $\mathrm{OD}_{595}$ after crystal violet staining of the MBEC peg lid. Bar charts represent the mean of three independent experiments with SD. The p-values were calculated using one-way ANOVA with Dunnets post hoc test to compare all treatments to the untreated control. $\left({ }^{* * *} \mathrm{p}=0.0001 ;{ }^{*} \mathrm{p}<0.05\right.$; ns= no significance) 


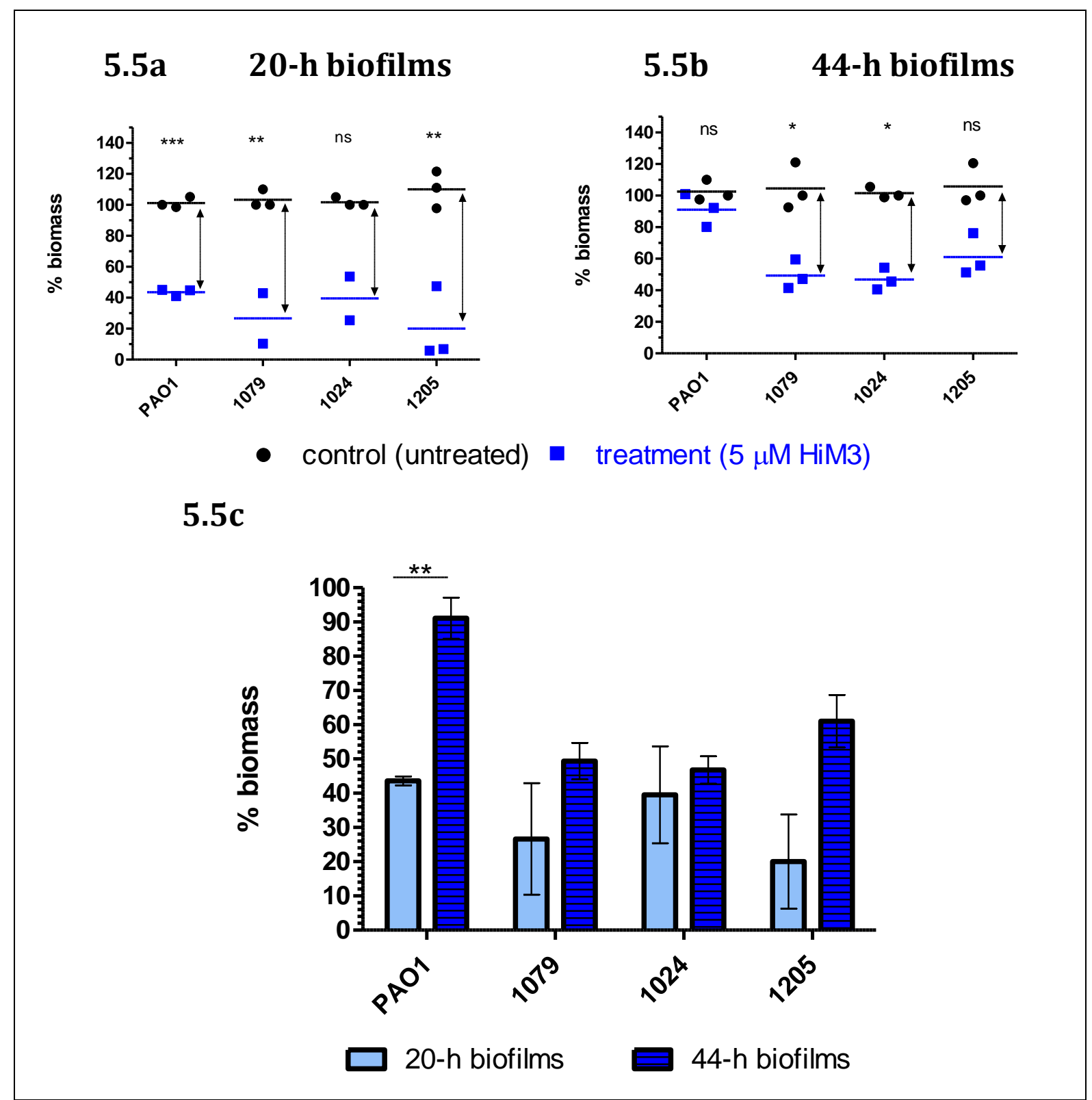

Figure 5.5 Effects of $5 \mu \mathrm{M}$ AgiM on 20-h and 44-h biofilms produced by P. aeruginosa

Bioflims produced by PA01, 1079, 1024 and 1205 were grown for 20-h (5a) or 44-h (5b), followed by treatment with $5 \mu \mathrm{M}$ AgiM for 6 -h. Disruption of biomass was determined by crystal violet staining and plotted as percentage growth normalised to the control. $\mathrm{P}$ values were calculated using one-way ANOVA with Dunnets post hoc test to compare all treatments to the untreated control. $\left({ }^{* *} \mathrm{p}=0.0001,{ }^{* *} \mathrm{p}=<0.01{ }^{*} \mathrm{p}<0.05\right.$; ns $=$ no significance). Figure $5 \mathrm{c}$ shows the comparison of biofilm mass reduction for 20 -h and 44 -h biofilms treated with $5 \mu \mathrm{M}$ AgiM in each strain. $P$ values were calculated using unpaired student $t$-test $\left({ }^{* *} p=0.015\right)$. 


\subsubsection{AgiM reduces the viability of $20-\mathrm{h}$ biofilms}

The bactericidal effect of AgiM was investigated on 20-h and 44-h biofilms by evaluating the number of viable cells remaining after treatment. This experiment was performed using only the laboratory strain PA01; biofilms were grown on MBEC devices, followed by AgiM treatment for 6-h. Individual pegs were removed from the MBEC device and placed into tubes for sonication to dissociate the biofilms and the number of colonyforming units (CFU) was determined by plating. A bactericidal agent exhibits 99.9\% killing, which can be defined as a $>3$ log reduction in CFU.

Analysis of 20 -h biofilms demonstrated AgiM treatment at concentrations of $2.5 \mu \mathrm{M}$ and $5 \mu \mathrm{M}$ caused a significant reduction in viable CFU compared to the control $(\mathrm{p}<0.05)$ (Figure 5.6a). The reduction of surviving cells was most significant at the highest concentration of $5 \mu \mathrm{M}$, which caused a 1-log fold $\mathrm{CFU} / \mathrm{mL}$ reduction compared to the control (Figure 5.6a). These data indicate AgiM reduces the number of surviving cells within 20 -h biofilms, but that the vast majority of cells remaining in the biofilm are still viable after treatment. These findings are consistent with the literature; Chambers et al. reported the enhanced tolerance of the biofilm mode of growth in a study of PAO1 biofilms, demonstrating exposure to tobramycin resulted in a 1.9-log reduction of cell viability compared to a 4.2-log reduction in viability of exponential-phase planktonic cells (80).

Treatment of 44-h biofilms with AgiM at all concentrations was ineffective, with no significant reduction in surviving cells compared to the control ( $p>0.05$ ) (Figure 6.6b). The enhanced tolerance exhibited by mature 44 -h biofilms is consistent with the literature, which suggests the maturation of biofilms coincides with increased resistance due to the biofilm matrix and phenotypic changes to cells increasing tolerance. 
Overall, these data show that AgiM can reduce the number of viable cells within biofilms, however, this effect is not bactericidal in the presence of sessile bacteria at the concentrations used in this experiment (10 x MIC). AgiM are not effective against more mature biofilms which exhibit more robust tolerance mechanisms.

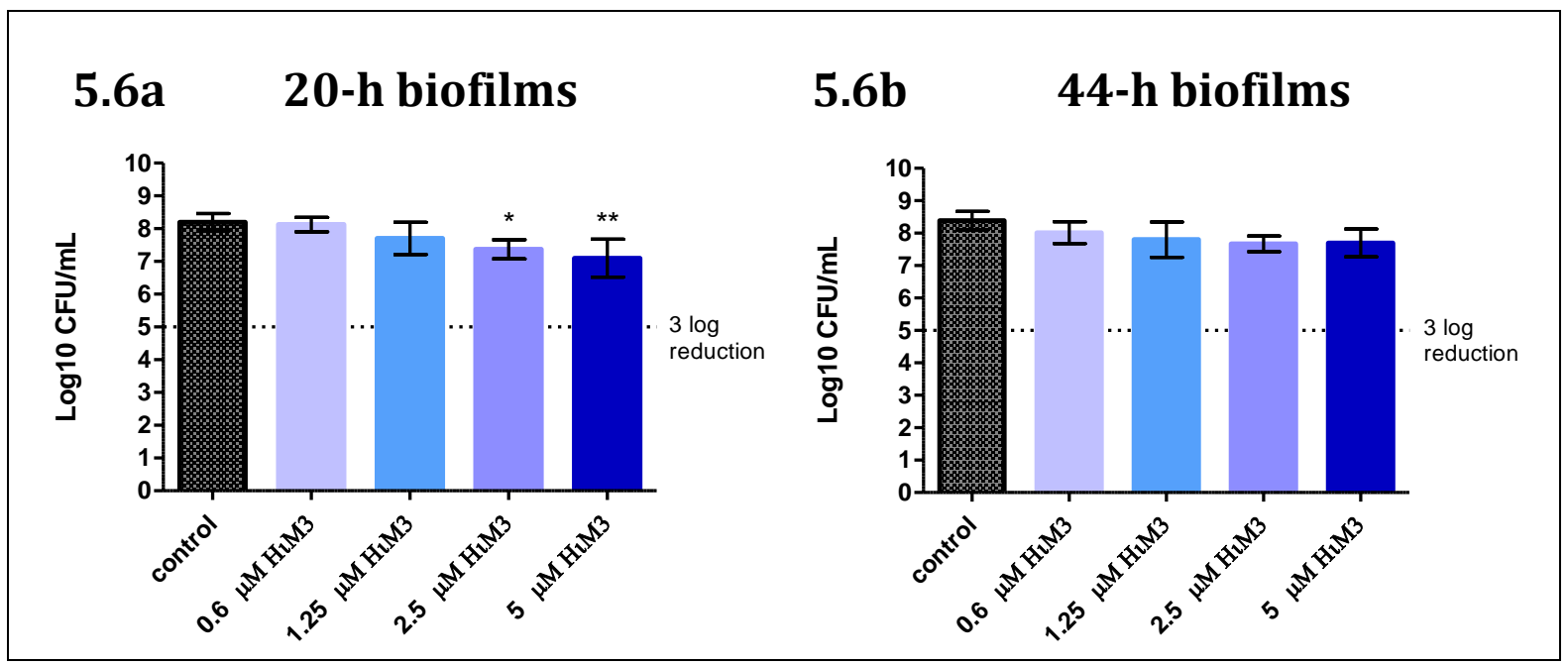

Figure 5.6 The effect of AgiM on 20-h and 44-h PA01 biofilm viability

The bar graphs represent two independent experiments, error bars show SD. n=2 independent experiments 


\subsection{Key findings}

This chapter demonstrated the antibiofilm activity of AgiMs against established $P$. aeruginosa biofilms, with inhibition of shed cell from the biofilm and disruption of the biofilm biomass. The experiments in this chapter showed strain-specific differences in susceptibility to AgiM treatment; however, all strains exhibited sensitivity to treatment.

Treatment of AgiMs killed a proportion of cells shed from biofilms, with a significant reduction in cells compared to the control in both 20 -h and 44 -h biofilms, 55\% and 54\% respectively (Figure 5.2). However, the fraction of killing observed was far less than expected with treatment concentrations exceeding the MIC $(10 \mathrm{x}$ MIC for PA01 and $5 \mathrm{x}$ MIC for clinical isolates). This discrepancy in sensitivity between cells shed from biofilms and planktonic cells in culture indicate cells released from biofilms exhibit higher tolerance than their planktonic counterparts.

The ability of AgiMs to disrupt biofilm biomass was demonstrated, with 20-h biofilms more susceptible than 44-h biofilms. Treatment with $5 \mu \mathrm{M}$ AgiM resulted in an average reduction in biomass across all strains of $68 \%$ in 20 -h biofilms and only $38 \%$ in 44 -h biofilms (Figure 5.4). AgiMs demonstrated the ability to disrupt biofilms; however, the cells remaining inside the biofilms remained highly resistant to treatment. Treatment with AgiMs reduced the number of surviving cells compared to the control to 20-h biofilms but was ineffective in the more resistant mature 44-h biofilms (Figure 5.6). The enhanced tolerance to AgiM challenge displayed by the mature 44-h biofilms is consistent with the literature showing established biofilms have inherent defence mechanisms such as a fully developed extracellular matrix and persister cell populations. 
An interesting finding from this study was that low concentrations of AgiMs have the potential to induce biofilm formation. Treatment with low concentrations caused an increase in shed cells from 20 -h and 44 -h biofilms, as well as inducing an increase in biomass for 44-h biofilms. While these increases were not statistically significant, it highlights the complexities of bacterial biofilm behaviour to antimicrobial therapy. Investigation of the antibiofilm activity of AgiMs demonstrated their potential for disruption and reduction of biofilms; however, it also highlighted the inherent difficulties of eradicating these bacterial communities due to tolerance and resistance mechanisms. 


\subsection{Discussion}

An interesting finding from this thesis was that cells shed from mature biofilms did not have the same susceptibility to AgiM treatment as planktonic cells. We anticipated shed cells to be susceptible to AgiMs, based on the notion that dispersed cells would return to the planktonic mode of growth. A proportion of shed cells were killed by AgiM treatment, but at concentrations far exceeding what is required to sterilise exponentially growing planktonic cells. This suggests shed cells from biofilms are not identical to their planktonic counterparts. There is precedence for the discrepancy in susceptibilities observed, with several reports that dispersed cells differ from both biofilm cells and planktonic cells, with a unique phenotype (80)(81)(18). It is proposed that cells shed from established biofilms retain a temporary tolerance, as they return to the planktonic mode of growth. Chambers and colleagues reported a 2-h period for dispersed cells to regain susceptibility to colistin treatment (80). This indicates that shed cells have a unique transitory phenotype with enhanced tolerance that is lost once they regain the planktonic mode of growth. However, the duration and mechanism involved in the phenotypic switch remain unclear.

The antibiofilm activity of AgiMs was demonstrated by the reduction of biofilm biomass in both immature and mature biofilms, with this effect less effective in better-established 44-h biofilms. Administration of a single dose of 5 x MIC AgiM over 6-h resulted in a $68 \%$ reduction of biofilm biomass in 24-h biofilms. The exceptional resistance displayed by bacterial biofilms requires treatment at concentrations that far exceed what is required to kill bacteria in the planktonic state. Bjarnsholt and colleagues demonstrated that 10 to 100 times more AgSD was required to kill P. aeruginosa biofilms compared to planktonic 
growth (79). It is hypothesised that increasing the concentration of AgiMs from $5 \mathrm{x}$ MIC to $10 \mathrm{x}$ MIC, acquainting to $10 \mu \mathrm{M}$ would be sufficient to eradicate the biofilm biomass.

The results of this chapter demonstrate a differential response to AgiM treatment within different subpopulations in the biofilm, with cells in the deeper layers of the biofilm tolerant to antimicrobial attack. The inability of AgiMs to significantly reduce the viability of remaining cells within the biofilm was attributed to the reduced penetration into the biofilm matrix and low diffusion through the layers of the biofilm likely resulting in subinhibitory concentrations of AgiM reaching the deeper layers of the biofilm structure. Tolerance in biofilms is associated with persister cell populations, a subset of bacteria that can endure extremely high antibiotic concentrations. Mah et al. reported a 20\% relapse of catheter-related bloodstream infections after an extended and aggressive treatment of colistin at concentrations up to 1000 -fold higher than the MIC over 14 days (77). These data indicate much higher concentrations of AgiM with repeated administration may be required to achieve the eradication of $P$. aeruginosa biofilms. Due to the strong biofilm biomass disruption activity of AgiM is it hypothesised that combination therapy of AgiMs with tobramycin could achieve sterilisation of biofilms. The synergy between these agents will be investigated in Chapter 6 .

The findings of this chapter indicated that sub-inhibitory levels of AgiM induce the formation of biofilms in P. aeruginosa, with an increase in biofilm biomass and viable CFU observed at low concentration of AgiM. These data are in complete agreement with the published literature describing biofilm stimulation by sub-inhibitory concentrations of antimicrobials (82). The first study to demonstrate the link between sub-MIC agents and biofilm formation was reported in 1988, with 0.25 x MIC rifampicin inducing biofilm formation in a strain of $S$. epidermidis (83). There is growing evidence that bacteria 
respond specifically and defensively to subinhibitory antibiotic concentrations, with Hoffman and colleagues finding administration of tobramycin at $0.3 \times$ MIC resulted in a 3.4-fold increase in the biomass of PA01 biofilms (84). The sub-inhibitory effect of biofilm induction appears to be ubiquitous across all types of antimicrobial and bacterial species (85) (82).

\subsubsection{Evaluation of experimental design and technique}

The experimental design used in this study aimed to provide a preliminary evaluation of the efficacy of AgiM on established biofilms. The rationale for this decision was the clinical relevance of established biofilms in the virulence of chronic $P$. aeruginosa infections, particularly in $\mathrm{CF}$ patients and the lack of antibacterial agents capable of biofilm eradication.

\subsubsection{Closed $v$ open system of biofilm growth}

Biofilms were established on MBEC devices, which are composed of a disposable 96-well microtiter plate with a lid that contains removable polystyrene pegs. The inoculated plate is incubated with agitation to allow cells to attach to the pegs and form biofilms. This offers a closed system of biofilm growth, with the advantages of simplicity, low cost and high throughput. A major disadvantage of this method is the risk of contamination from manipulation of pegs during removal for analysis. Further limitations include the potential of loosely attached biofilms becoming dislodged from pegs during the multiple washing steps and the difficultly removing firmly attached biofilms through sonication

Comparatively, the open system of biofilm growth using a flow cell is one of the most commonly performed techniques as it allows for control of growth parameters and dynamics. This method sees bacteria inoculums injected through silicone tubing into flow cells containing a nutrient medium. Cells attach to a surface, usually microscopy 
coverslips that allow the biofilm formation to be observed. The major advantage of this open system of biofilm growth is the potential for pharmacokinetic and pharmacodynamic assessments, as well as the exclusion of excludes any remaining planktonic cells which are eliminated by the constant flow. It also allows for nondestructive observation of the formation and maturation of biofilms using confocal laser scanning microscopy. The major disadvantage of this method is its time-consuming nature, taking several days to set up and maintain and can also be costly and complex to perform.

\subsubsection{Evaluation of biofilm biomass}

The crystal violet assay used in this study is among the most frequently used assays for investigating the effects of agents on biofilm biomass (Azerado et al., 2017). The limitation of this technique is that crystal violet stains viable cells and dead cells remaining in the biofilm matrix, providing an evaluation of biomass but not specifically the killing of the biofilm. The main advantages of this method are its reproducibility and rapid analysis of biofilm reduction. Alternative options, such as direct measurement of biomass using confocal microscopic quantification, can be labour intensive and expensive in comparison. However, the use of confocal laser scanning microscopy (CLSM) is an indispensable tool that allows direct visualisation of the differential killing of a subpopulation within each layer of the biofilm over the course of treatment and should be considered for future optimisation of AgiM investigations.

\subsubsection{Determination of biofilm viability}

Determination of viability by enumeration of colony-forming units on agar media is the most widely used technique and is a relatively simple and cost-effective method. The disadvantage of this method, aside from its time-consuming nature, is the serious 
limitation in detection as it detects only the culturable fraction of cells in the biofilm. The proportion of dormant, viable, but non-culturable cells in the biofilm are not detected (Li et al., 2014). An alternative method to be considered for future experiments is the use of flow cytometry to accurately determine the viability of biofilm cells (Cerca et al., 2011). Due to the expense of this technique, it was not appropriate for preliminary studies of AgiM.

\subsubsection{Limitations of in vitro biofilm susceptibility testing}

A major challenge for researchers is the development of standardised biofilm susceptibly testing assays and interpretation parameters to facilitate the correlation between in vitro results and treatment outcome. Classic in vitro susceptibility parameters such as MIC, aimed to predict therapeutic success are not applicable for biofilms; demonstrated in this study, by AgiM concentrations at 10 x MIC unable to kill bacteria in biofilms. Accordingly, pharmacodynamic parameters to quantify antimicrobial activity in biofilms have been established but are yet to have standardised biofilm endpoint parameters defined by EUCAST or CLSI (Thieme et al., 2019). Similar to the MIC, the minimum biofilm inhibitory concentration (MBIC) is defined as the lowest concentration of drug that results in an OD 650 difference of $\leq 10 \%$ of the mean of two positive control well readings. The biofilm bactericidal concentration (BBC) is the lowest concentration of drug that killed $99.9 \%$ of the cells recovered from a biofilm culture compared to the control, and the minimal biofilm-eradication concentration (MBEC) is defined as the lowest concentration of drug required to eradicate the biofilm (Joo et al., 2002).

Another limiting factor in susceptibility testing of biofilms is the current disparity between in vitro and in vivo results. The flow cell system is the most comparable method but is not realistic for high throughput routine testing. Optimal growth conditions and 
media need to be established to better reproduce the in vivo conditions. Additionally, there is a lack of consistent and robust animal biofilm models. 


\section{CHAPTER 6. AGIMS AND SYNERGY AGAINST}

\section{P. AERUGINOSA}

\subsection{Aims and rationale}

The emergence and escalation of MDR resistant strains of $P$. aeruginosa with the ability to acquire resistance to all known antibiotics is an urgent global health concern. Recent efforts to meet this rising challenge have led to the use of antibiotic adjuvants and synergistic combinations to resuscitate the potency of the current clinical arsenal. Adjuvant agents potentiate the activity of antibiotics to regain susceptibility, while synergy between antimicrobial agents lowers the required concentration of antimicrobials to kill the pathogen (72). Combination therapy, whereby two or more antibacterial agents are combined, can be used to combat severe infections combined to enhance the clinical efficacy (86). Additionally, this enhanced efficacy allows the use of a lower dose reducing the risk of adverse effects.

Silvers ability to disrupt multiple bacterial cellular processes, including increased membrane permeability in Gram-negative bacteria leads to the ability to potentiate the activity of several different classes of antibiotics. There are multiple reported of silver displaying synergistic activity with several antibiotics to restore drug sensitivity in $E$. coli (50) and against several resistant strains of Gram-negative bacteria, including $P$. aeruginosa (87).

Therefore, it was hypothesised that $\mathrm{Ag}^{+}$in the form of AgiM may exhibit synergistic activity with tobramycin, a commonly used aminoglycoside for P. aeruginosa infections. The aim of this chapter was to investigate the possible synergistic interactions between 
AgiM HiM3 and tobramycin. This would have the potential clinical advantage of reducing adverse toxic effects and enhancing the efficacy of tobramycin in resistant $P$. aeruginosa infections.

\subsection{Methods}

The checkerboard microdilution method was used to investigate the interactions between antimicrobial agents AgiM and tobramycin (see Materials and Methods 2.10). At the completion of the checkerboard assay, the fractional inhibitory concentration (FIC) index was determined for each dilution that showed no bacterial growth $\left(\mathrm{OD}_{600}<0.02\right)$, using the formula SFIC $=$ FIC tobramycin + FIC Agim .

$$
\begin{gathered}
\text { FIC tobramycin }=\text { MIC combination } / \mathrm{MIC}_{\text {tobramycin alone }} \\
\text { FIC }_{\text {AgiM }}=\mathrm{MIC}_{\text {combination }} / \mathrm{MIC}_{\text {AgiM alone }}
\end{gathered}
$$

The FIC indices were calculated according to the method of Elioponlo and Moerllering, with the results interpreted as; synergy $(\leq 0.5)$, partial synergy/additive $(0.5-1.0)$, indifference (1.0 - 4.0) and antagonism (>4.0) (Erzsele et al. 2015). The data was also presented visually on isobologram plots to confirm and analyse the interactions between tobramycin and AgiM. The isobologram is a commonly used method to visualise effective dose pairs (isoboles) graphically (88). The MIC values of single agents and different ratio combinations were plotted as axial points in a Cartesian plot. A straight line connects the MIC of tobramycin and AgiM, representing doses that produce an additive combination. This line of additivity allows comparison with the effective dose pairs plotted from experimental data. Dose pairs below this line are considered synergistic, and above are antagonistic. 


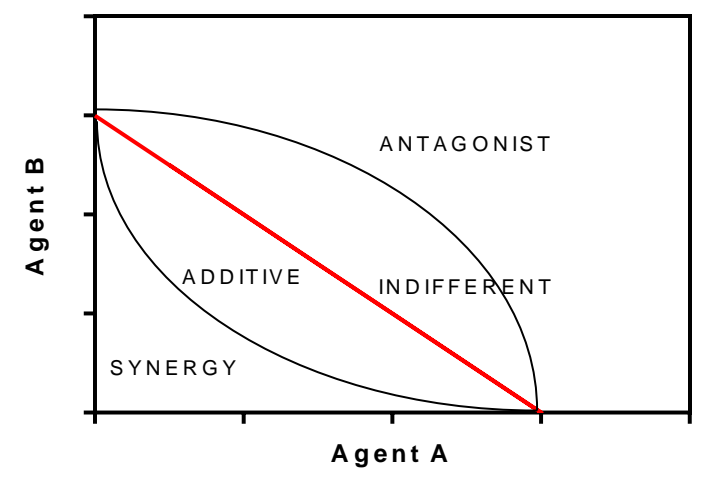

Figure 6.1 Schematic of Isobologram graph. The line of additivity drawn between the MIC of both agents is in red. The area above this line indicates indifferent, and antagonist interactions, and below this line indicate additive and synergistic interactions. 


\subsection{Results}

\subsubsection{AgiMs synergise with tobramycin}

The MIC of AgiMs HIM3 and tobramycin was determined for each strain of P. aeruginosa to allow appropriate concentrations for checkerboard assembly. All four strains showed equal sensitivity to tobramycin and AgiMs (Table 6-1). The MIC of tobramycin at 1.5 $\mu \mathrm{g} / \mathrm{mL}$ is in accordance with published values (MIC $<5 \mu \mathrm{g} / \mathrm{mL}$ ) for susceptible strains of P. aeruginosa (72). It was unexpected that all strains displayed equal sensitivity to AgiM treatment, as previous experiments in this study found PAO1 to be more sensitive than clinical isolates with an MIC of $0.5 \mu \mathrm{M}$ (Table 4-1). This variation could be attributed to bacterial variation between inoculums used in these experiments.

Table 6-1 in vitro sensitivities of P. aeruginosa strains to antimicrobial agents AgiM and tobramycin

\begin{tabular}{|c|cc|}
\hline P. AERUGINOSA STRAIN & MIC OF TOBRAMYCIN & MIC OF AGIM \\
\hline PAO1 & $1.5 \mu \mathrm{g} / \mathrm{mL}$ & $1 \mu \mathrm{M}$ \\
$\mathbf{1 2 0 5}$ & $1.5 \mu \mathrm{g} / \mathrm{mL}$ & $1 \mu \mathrm{M}$ \\
$\mathbf{1 0 2 4}$ & $1.5 \mu \mathrm{g} / \mathrm{mL}$ & $1 \mu \mathrm{M}$ \\
$\mathbf{1 0 7 9}$ & $1.5 \mu \mathrm{g} / \mathrm{mL}$ & $1 \mu \mathrm{M}$ \\
\hline
\end{tabular}

The interaction of AgiM and tobramycin combined was assessed by FIC indices, which ranged from 0.37 (synergy) to 1.05 (indifference) across the four strains tested (Table 62). All strains showed partial synergy, with PA01, 1205 and 1024 showing full synergy at specific concentrations. There were no antagonistic interactions across any of the strains examined.

The most effective treatment pairs to achieve synergy in the PA01 strain was $0.18 \mu \mathrm{g} / \mathrm{mL}$ tobramycin combined with $0.25 \mu \mathrm{M}$ AgiM. These doses are significantly less than when used alone. Tobramycin had a significant 8-fold decrease from $1.5 \mu \mathrm{g} / \mathrm{mL}$ to $0.18 \mu \mathrm{g} / \mathrm{mL}$, and AgiM had a 4-fold decrease from $1 \mathrm{uM}$ to $0.25 \mu \mathrm{M}$. For clinical isolates 1205 and 1024, 
the effective combination of $0.37 \mu \mathrm{g} / \mathrm{mL}$ tobramycin and $0.25 \mu \mathrm{M}$ AgiM achieved synergy.

This showed a 4-fold decrease in the concentration of antimicrobial agents required compared to when used alone.

Table 6-2 FIC calculations and interpretations for all strains of $P$. aeruginosa determined from checkerboard assay . synergy $(\leq 0.5)$, partial synergy $(0.5-1.0)$, indifference $(1.0-4.0)$, antagonism (>4.0). Synergistic combinations are highlighted in blue.

\begin{tabular}{|c|c|c|c|c|}
\hline $\begin{array}{c}\text { Tob/AgiM } \\
\text { Combination }\end{array}$ & $\begin{array}{c}\text { FIC }_{\mathbf{A}} \\
\mathrm{MIC}_{\mathrm{A} \text { combo }} / \mathrm{MIC}_{\mathrm{Tob}}\end{array}$ & $\begin{array}{c}\text { FIC }_{\mathbf{B}} \\
\mathrm{MIC}_{\mathrm{B}} \\
\text { combo/MIC } \mathrm{MIg}_{\mathrm{Ag}}\end{array}$ & $\begin{array}{l}\sum F I C= \\
\text { FIC }_{A}+\text { FIC }_{B}\end{array}$ & Effect \\
\hline \multicolumn{5}{|l|}{ PA01 } \\
\hline $0.07 / 0.5$ & $0.07 / 1.5$ & $0.5 / 1$ & 0.55 & $\begin{array}{l}\text { PARTIAL } \\
\text { SYNERGY }\end{array}$ \\
\hline $0.18 / 0.25$ & $0.18 / 1.5$ & $0.25 / 1$ & 0.37 & SYNERGY \\
\hline $0.37 / 0.25$ & $0.37 / 1.5$ & $0.25 / 1$ & 0.49 & SYNERGY \\
\hline $0.75 / 0.125$ & $0.37 / 1.5$ & $0.125 / 1$ & 0.625 & $\begin{array}{l}\text { PARTIAL } \\
\text { SYNERGY }\end{array}$ \\
\hline \multicolumn{5}{|l|}{1024} \\
\hline $0.07 / 0.5$ & $0.07 / 1.5$ & $0.5 / 1$ & 0.55 & $\begin{array}{l}\text { PARTIAL } \\
\text { SYNERGY }\end{array}$ \\
\hline $0.18 / 0.5$ & $0.18 / 1.5$ & $0.5 / 1$ & 0.62 & $\begin{array}{l}\text { PARTIAL } \\
\text { SYNERGY }\end{array}$ \\
\hline $0.37 / 0.25$ & $0.37 / 1.5$ & $0.25 / 1$ & 0.49 & SYNERGY \\
\hline $0.75 / 0.125$ & $0.75 / 1.5$ & $0.125 / 1$ & 0.625 & $\begin{array}{l}\text { PARTIAL } \\
\text { SYNERGY }\end{array}$ \\
\hline \multicolumn{5}{|l|}{1079} \\
\hline $0.07 / 0.5$ & $0.07 / 1.5$ & $0.5 / 1$ & 0.55 & $\begin{array}{l}\text { PARTIAL } \\
\text { SYNERGY }\end{array}$ \\
\hline $0.18 / 0.5$ & $0.18 / 1.5$ & $0.5 / 1$ & 0.62 & $\begin{array}{l}\text { PARTIAL } \\
\text { SYNERGY }\end{array}$ \\
\hline $0.37 / 0.5$ & $0.37 / 1.5$ & $0.5 / 1$ & 0.75 & $\begin{array}{l}\text { PARTIAL } \\
\text { SYNERGY }\end{array}$ \\
\hline $0.75 / 0.25$ & $0.75 / 1.5$ & $0.25 / 1$ & 0.75 & $\begin{array}{l}\text { PARTIAL } \\
\text { SYNERGY }\end{array}$ \\
\hline \multicolumn{5}{|l|}{1205} \\
\hline $0.07 / 1$ & $0.07 / 1.5$ & $1 / 1$ & 1.05 & INDIFFERENT \\
\hline $0.18 / 0.5$ & $0.18 / 1.5$ & $0.5 / 1$ & 0.62 & $\begin{array}{l}\text { PARTIAL } \\
\text { SYNERGY }\end{array}$ \\
\hline $0.37 / 0.25$ & $0.37 / 1.5$ & $0.25 / 1$ & 0.49 & SYNERGY \\
\hline $0.75 / 0.125$ & $0.75 / 1.5$ & $0.125 / 1$ & 0.62 & $\begin{array}{l}\text { PARTIAL } \\
\text { SYNERGY }\end{array}$ \\
\hline
\end{tabular}


The isobolograms acquired from the checkerboard assay demonstrated no antagonistic effect between the two antimicrobials. All interactions were illustrated to be additive or synergistic, except for one indifferent interaction in strain 1205 (Figure 6.2). Results from this experiment confirm synergy can be achieved by the combination of AgiM and tobramycin at doses significantly lower than the MIC. These data indicate that treatment with AgiM and tobramycin in combination can exhibit significant synergistic toxicity on bacterial cells.

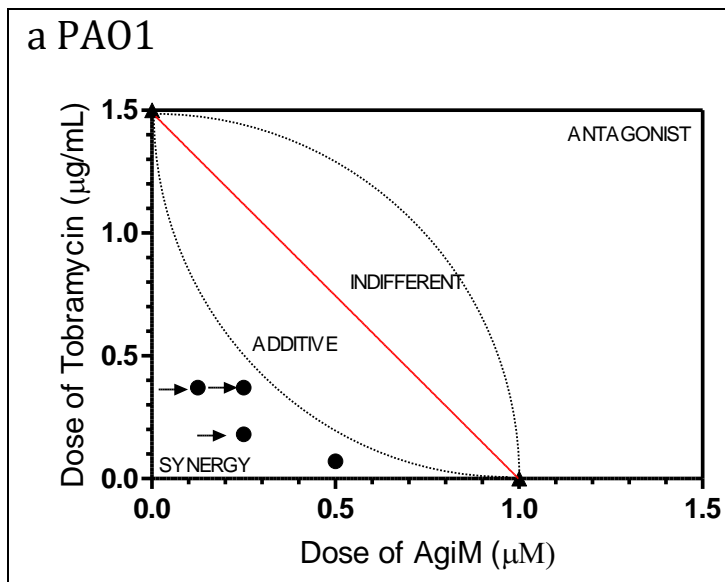

\section{b 1205}

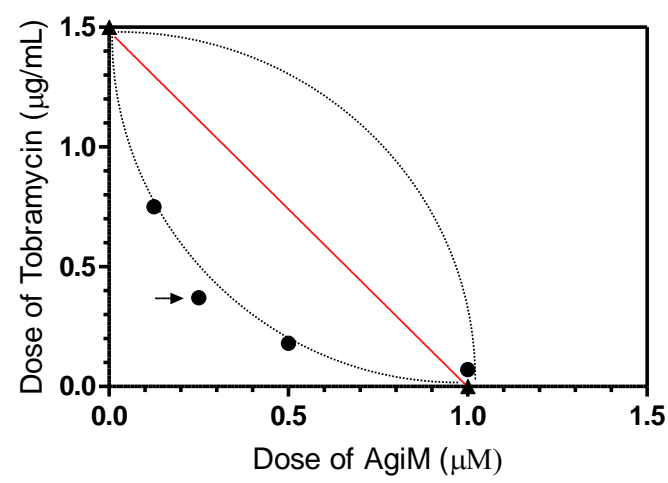

d 1024
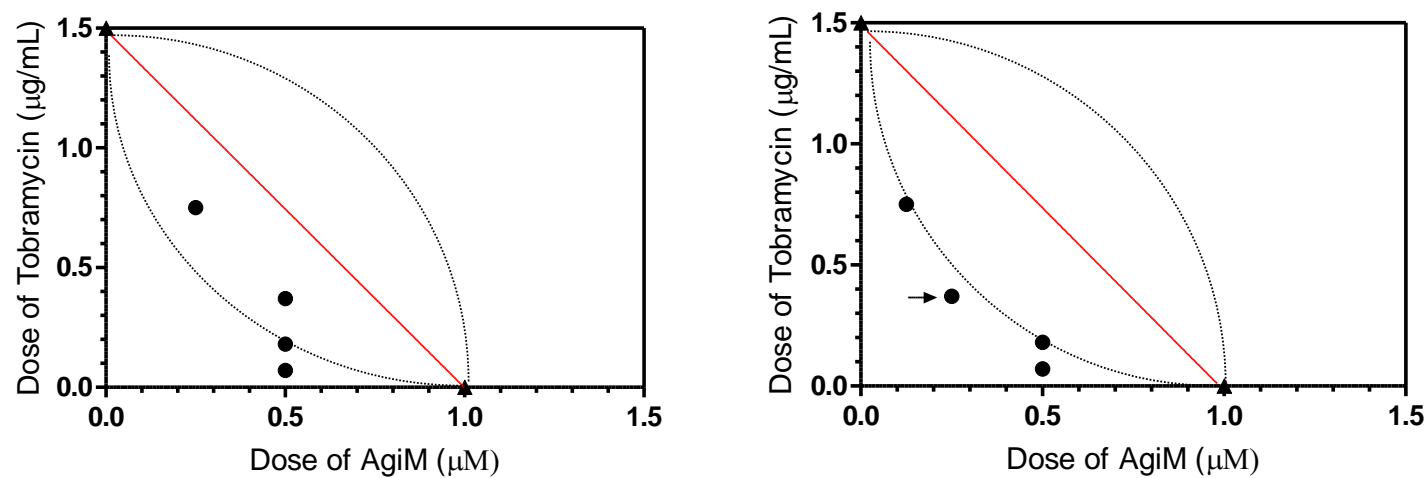

Figure 6.2 a-d: Isobolograms confirm synergistic and additive interactions between AgiM and tobramycin in four strains of $P$. aeruginosa.

(A) PA01, (B) clinical isolate 1205, (C) clinical isolate 1079, (D) clinical isolate 1024. Figure 2A is annotated to demonstrate regions of synergy, additive, indifferent and antagonist interaction, as illustrated in Figure 1. Arrows point to treatment pairs that achieved full synergy (FIC>0.5). A straight red line connects the MIC values of tobramycin and AgiM alone. Effective treatment pairs from checkerboard assays were plotted as axial points. Points below the red line indicate additive or synergistic interactions. 


\subsection{Key findings}

The synergy between two antibacterial agents is defined as the effect elicited by two agents used in combination that is greater than the sum of the effects elicited by the compounds used individually (88). The results of this chapter confirm the hypothesis that AgiMs in combination with tobramycin exhibit synergistic activity. Analysing the FIC values across the four strains tested showed a mean FIC of 0.61 that describes a partially synergistic effect of the combination. Full synergy $(\mathrm{FIC}<0.5)$ was achieved in three strains PA01, 1205 and 1024; thus, the overall interaction of partial synergy could be due to the low number of strains investigated in this study. No antagonistic interactions were observed in any of the strains, suggesting this combination has the potential for further in vitro and in vivo investigation.

The findings of this chapter are in accordance with the numerous publications that have reported synergy between $\mathrm{Ag}^{+}$with multiple classes of antibiotics such as tetracyclines, quinolones, $\beta$-lactams and aminoglycosides (50) (51). These studies concluded that aminoglycosides demonstrate the highest potential to synergise with $\mathrm{Ag}^{+}(51)(50)$. Aminoglycosides, a bactericidal class of antibiotic, bind the $16 \mathrm{~S}$ rRNA component of the 30 S ribosomal subunit in bacteria to induce protein mistranslation through the misincorporation of amino acids contributing to cell death (51). A crucial step in the aminoglycoside mode of action is to be transported across the bacterial cytoplasmic membrane in order to reach its target. P. aeruginosa is known to confer resistance to aminoglycosides by alteration of the outer membrane composition. It is predicted that AgiMs potentiate the activity of tobramycin by $\mathrm{Ag}^{+}$induced disruption of the outer membrane allowing enhanced cellular penetration of tobramycin into the bacterial cell. 


\subsection{Discussion}

While the exact molecular mechanism behind the synergistic interactions between silver and aminoglycosides are yet to be fully understood, the overall consensus is that $\mathrm{Ag}^{+}$acts upon multiple molecular targets to destabilise and weaken the bacterial membrane, ultimately leading to a massive increase in antibiotic uptake (51), (50).

Silvers potentiating effect on aminoglycoside activity to kill persistor cells and inhibit biofilm formation has been reported. Both persistor cells and biofilm formation are indicative of resistance in Gram-negative organisms. A combination of sublethal $\mathrm{Ag}^{+}$plus gentamicin on E. coli persistor cells and an in vitro E. coli biofilm exhibited increased bactericidal activity compared to individual treatment with $\mathrm{Ag}^{+}$or the antibiotic alone in both experimental models (50). These findings suggest AgiMs could be used synergistically with aminoglycosides to treat chronic $P$. aeruginosa infections, which exbibit strong resistance due to biofilm formation.

This thesis investigated the in vitro potential for synergy between AgiMs and tobramycin; however, there is evidence of in vivo synergistic interactions between silver and aminoglycosides that indicate future investigations of AgiMs in animal models are warranted. The effect of treatment with $\mathrm{Ag}^{+}$and vancomycin, individually and combined in both an acute and mild peritonitis mouse model showed that combined treatment of vancomycin plus $\mathrm{Ag}^{+}$led to a significant reduction in E. coli cell counts in both models. Additionally, treatment of vancomycin plus $\mathrm{Ag}^{+}$increased survival in the acute model, with $90 \%$ survival after five days compared to only $50 \%$ survival of mice treated with $\mathrm{Ag}^{+}$only, and $10 \%$ survival in mice treated with vancomycin only (50).

This study shows the in vitro potential for synergy between AgiMs and tobramycin in four susceptible strains of $P$. aeruginosa. Synergistic interactions allow for lower or sublethal 
doses of antibacterial agents, conferring multiple benefits, including delaying the emergence of resistance and reducing drug-related toxicity due to the decreased dose required to achieve potency. The interaction between AgiMs and tobramycin demonstrates the use of sublethal doses of both agents can be clinically effective; offering the potential for the systemic use of AgiMs in a clinical setting feasible. 



\section{CHAPTER 7. INVESTIGATION OF IN VIVO ACTIVITY OF}

\section{AGIMS}

\subsection{Aims and rationale}

The use of mammalian models of infection during antimicrobial testing is essential to determine the efficacy of treatments and to evaluate advancement to other models of infection or commencement of human clinical trials. The larvae of the greater wax moth Galleria Mellonella were used in this thesis to investigate the virulence of the Gramnegative bacteria P. aeruginosa and to evaluate the antibacterial efficacy of AgiMs. This well-established model of infection has been developed for a variety of human pathogens, including but not limited to $P$. aeruginosa (89), A. baumannii (90), and Listeria monocytogenes (91). The larvae of G. Mellonella are a good proxy for human infection; while they lack an adaptive immune response, the larvae possess an innate immune system comparable to mammals in structure and function (92). The aim of this chapter was to investigate the ability of AgiMs to ameliorate $P$. aeruginosa infections in a larval model, with both laboratory and clinical isolates of $P$. aeruginosa evaluated. It was hypothesised that administration of AgiMs would prolong survival of infected larvae in a dose-dependent manner, correlating with the in vitro antibacterial activity reported in this thesis. 


\subsection{Results}

\subsubsection{G. Mellonella are highly susceptible to infection by $P$. aeruginosa}

P. aeruginosa is highly virulent in G. Mellonella, with doses as small as 25 CFU of strain NCTC13437 achieving $100 \%$ mortality of larvae within 24-h (89). Initial experiments aimed to determine the appropriate number of CFU that will not kill the larvae immediately but lead to increased mortality over the duration of the experiment enabling monitoring of survival over this time. The appropriate bacterial inoculum was defined in this study as 80 - $100 \%$ mortality within $24-48 \mathrm{~h}$.

Groups of 10 larvae were infected with a range of bacterial inoculums, $\left(2 \times 10^{3} \mathrm{CFU} / \mathrm{mL}\right.$, $4 \times 10^{3} \mathrm{CFU} / \mathrm{mL}, 6 \times 10^{3} \mathrm{CFU} / \mathrm{mL}, 8 \times 10^{3} \mathrm{CFU} / \mathrm{mL}$ and $10 \times 10^{3} \mathrm{CFU} / \mathrm{mL}$ ) and incubated at $37^{\circ} \mathrm{C}$; with survival monitored over 48 -h. Table $7-1$ shows that each P. aeruginosa strain had different susceptibilities, with clinical isolate 1079 the most virulent and requiring only $20 \mathrm{CFU} /$ larva compared to the less virulent isolates 1205 and 1024 that required $100 \mathrm{CFU} /$ larva to reach the defined 80 - $100 \%$ mortality.

The effect of infection with P. aeruginosa PA01, 1079, 1205 and 1024 on the survival of G. Mellonella larvae is shown in Figure 7.1a-d. These data show G. Mellonella larvae are susceptible to infection by $P$. aeruginosa with all larvae infected at the highest bacterial concentrations $\left(8 \times 10^{3} \mathrm{CFU} / \mathrm{mL}\right.$ and $\left.10 \times 10^{3} \mathrm{CFU} / \mathrm{mL}\right)$ showing $100 \%$ mortality at the completion of the experiment (grey dashed line and black solid line, Figure 7.1a-d). Larvae injected with PBS-only (uninfected control) showed 100\% survival, confirming the manipulation itself did not cause harm to the larvae.

G. Mellonella were highly susceptible to infection by strain 1079 , showing $100 \%$ mortality by 17 -h with doses $4 \times 10^{3} \mathrm{CFU} / \mathrm{mL}, 8 \times 10^{3} \mathrm{CFU} / \mathrm{mL}$ and $10 \times 10^{3} \mathrm{CFU} / \mathrm{mL}$, and by 21 -h at $2 \times 10^{3} \mathrm{CFU} / \mathrm{mL}$ (Figure 7.1b). The laboratory strain PA01 showed $80 \%$ larval 
mortality at 20-h after infection with $4 \times 10^{3} \mathrm{CFU} / \mathrm{mL}$ (Figure 7.1a). Larvae were less susceptible to infection by strains 1205 and 1024 and only reached $80 \%$ mortality at the highest inoculum of $10 \times 10^{3} \mathrm{CFU} / \mathrm{mL}$ (Figure 7.1c,d). The defined bacterial inoculum for each strain is listed in Table 1, and will be used in subsequent experiments in this chapter.

These data indicate G. Mellonella larvae are highly susceptible to infection with $P$. aeruginosa strains used in this study, in a dose-dependent manner. The inoculum range of $20 \mathrm{CFU}$ to $100 \mathrm{CFU}$ necessary to achieve mortality demonstrates the variability in virulence of different strains of $P$. aeruginosa.

Table 7-1: Summary of inoculum size required to achieve $\geq \mathbf{8 0 \%}$ death of larvae within 24 $48 \mathrm{~h}$ post-infection in different strains of $P$. aeruginosa.

These indicate the determined CFU per larvae to be used in future experiments.

\begin{tabular}{|c|c|c|}
\hline P. aeruginosa strain & $\begin{array}{c}\text { Bacterial load to achieve } \\
\geq \mathbf{8 0} \% \text { mortality }\end{array}$ & CFU per larvae \\
\hline PAO1 & $4 \times 10^{3} \mathrm{CFU} / \mathrm{mL}$ & 40 \\
\hline 1079 & $2 \times 10^{3} \mathrm{CFU} / \mathrm{mL}$ & 20 \\
\hline 1205 & $10 \times 10^{3} \mathrm{CFU} / \mathrm{mL}$ & 100 \\
\hline 1024 & $10 \times 10^{3} \mathrm{CFU} / \mathrm{mL}$ & 100 \\
\hline
\end{tabular}




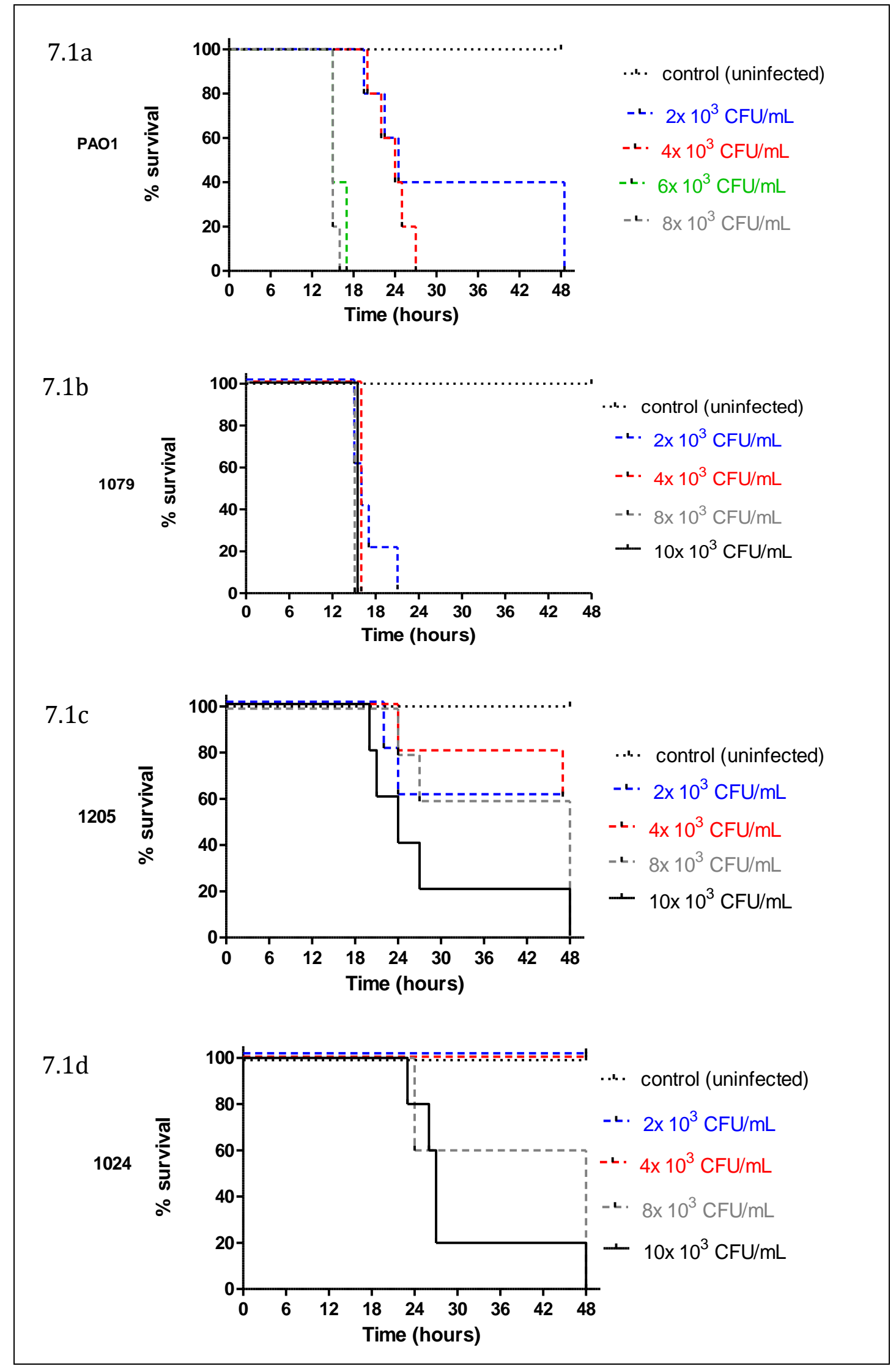

Figure 7.1 Effect of varying inoculum doses of $P$. aeruginosa strains on the survival of $G$. mellonella larvae.

$7.1 \mathrm{a}-\mathrm{d}$ : Instar larvae were infected with a range of bacterial concentrations $\left(2 \times 10^{3}\right.$ $\mathrm{CFU} / \mathrm{mL}, 40 \times 10^{3} \mathrm{CFU} / \mathrm{mL}, 60 \times 10^{3} \mathrm{CFU} / \mathrm{mL}, 80 \times 10^{3} \mathrm{CFU} / \mathrm{mL}, 100 \times 10^{3} \mathrm{CFU} / \mathrm{mL}$ ) for 
each strain and survival recorded over 48 hours during incubation at $37^{\circ} \mathrm{C}$. The control group were injected with PBS only. $n=20$ (pooled from 2 independent experiments for each strain). 


\subsubsection{Treatment with AgiMs extends survival of G. mellonella larvae after infection with $P$. aeruginosa}

\subsubsection{AgiM treatment of $P$. aeruginosa PA01 exhibits a dose-dependent increase in larval survival}

Larvae were infected with $4 \times 10^{3} \mathrm{CFU} / \mathrm{mL}$ (40 CFU) PA01 followed by treatment of AgiM HiM3 20 mins post-infection at concentrations of $10 \mu \mathrm{M}, 5 \mu \mathrm{M}$ and $2.5 \mu \mathrm{M}$, then incubated at $37^{\circ} \mathrm{C}$ and monitored hourly for survival. Control groups that were not infected with bacteria, but administered with AgiM-only or PBS-only showed $100 \%$ survival; demonstrating the manipulation and treatment itself did not cause mortality. Two independent experiments, performed one month apart using different batches of larvae are presented as Kaplan-Meier survival curves in Figures $7.2 \mathrm{a}$ and $7.2 \mathrm{~b}$. In both independent experiments, all doses of AgiM extended survival of larvae compared to the untreated control. The lowest dose of $2.5 \mu \mathrm{M}$ prolonged survival, but to a lesser extent than the highest dose of $10 \mu \mathrm{M}$. Both experiments show the same trend, increasing doses of AgiMs results in increasing survival. Panel $2 \mathrm{~b}$ showed higher mortality in the control compared to panel 7.2a most likely due to variation in susceptibility between batches.

Figure 7.2c shows the combined survival percentages of larvae at 4-h post onset of mortality in the untreated control. These data show all concentrations increase survival, with $10 \mu \mathrm{M}$ AgiM significantly extending survival to $90 \%$ compared to the untreated control at $40 \%(\mathrm{p}<0.05)$ (Figure 7.2c).

An increase in survival was observed after AgiM treatment (figure 2a) with increasing statistical significance between the untreated control and $2.5 \mu \mathrm{M}, 5 \mu \mathrm{M}$ and $10 \mu \mathrm{M}$ treatment groups determined by Log Rank (Mantel-Cox) test (at least $\mathrm{p}<0.05$ ). Variation between experimental repetitions was observed, the untreated control in 2 a reached $90 \%$ mortality at 24 -h post-infection, compared to $2 \mathrm{~b}$, which achieved $100 \%$ mortality 
of the control larvae at 22-h post-infection. Analysis of larvae survival 4 hours after the onset of mortality allowed further characterisation of survival differences. Figure 7.2c showed a dose-dependent increase in survival, with treatment of $10 \mu \mathrm{M}$ AgiM significantly increasing larval survival proportion compared to the untreated control determined by one-way ANOVA $(\mathrm{p}=0.02)$ (figure $7.2 \mathrm{c}$ ).

Overall, these data demonstrate AgiM treatment prolongs survival of larvae infected with PA01 in a dose-dependent manner. 
$7.2 \mathrm{a}$

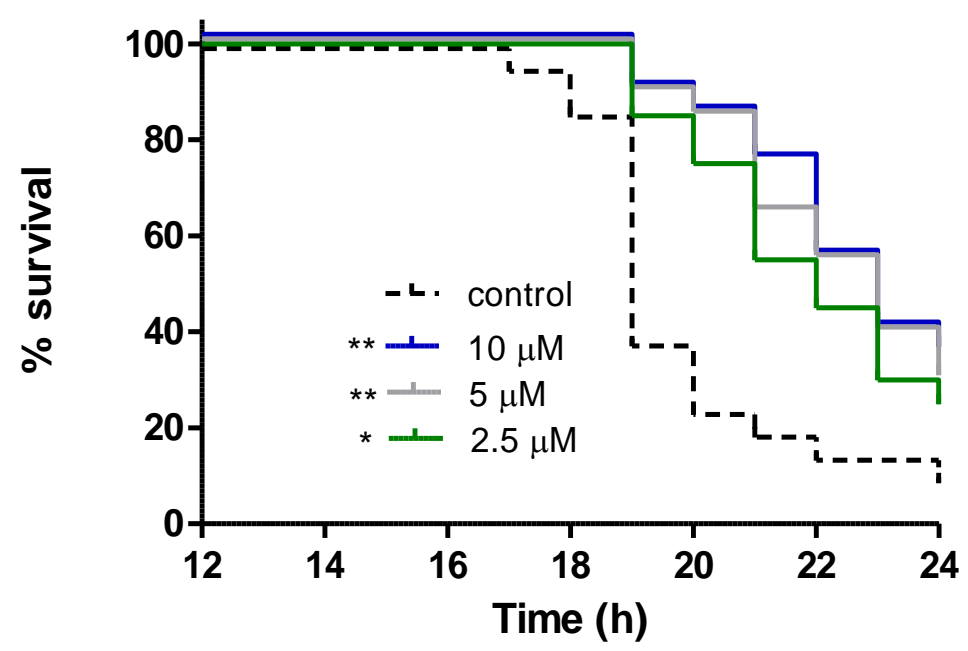

$7.2 \mathrm{~b}$

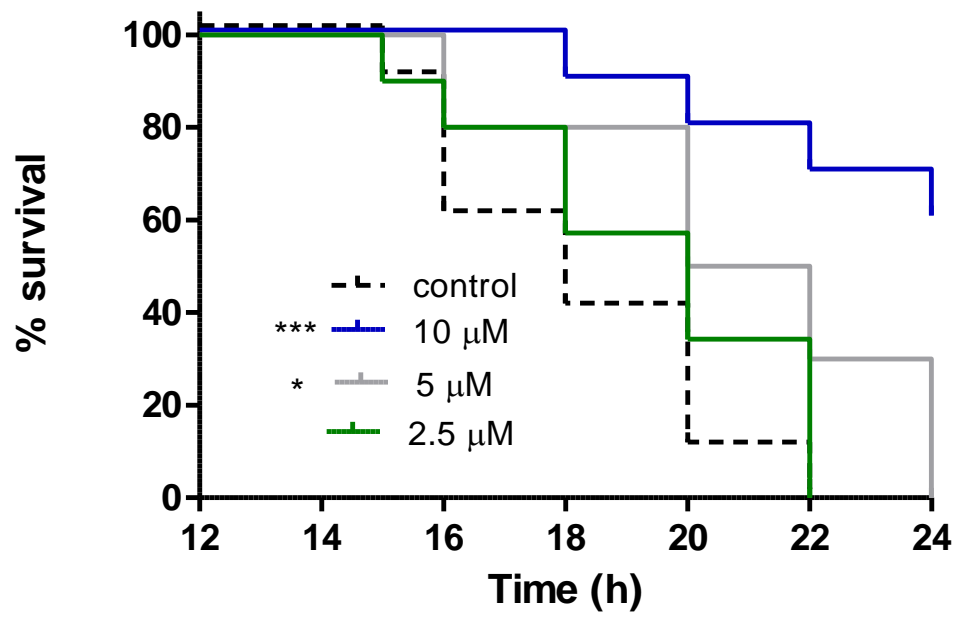

$7.2 \mathrm{c}$

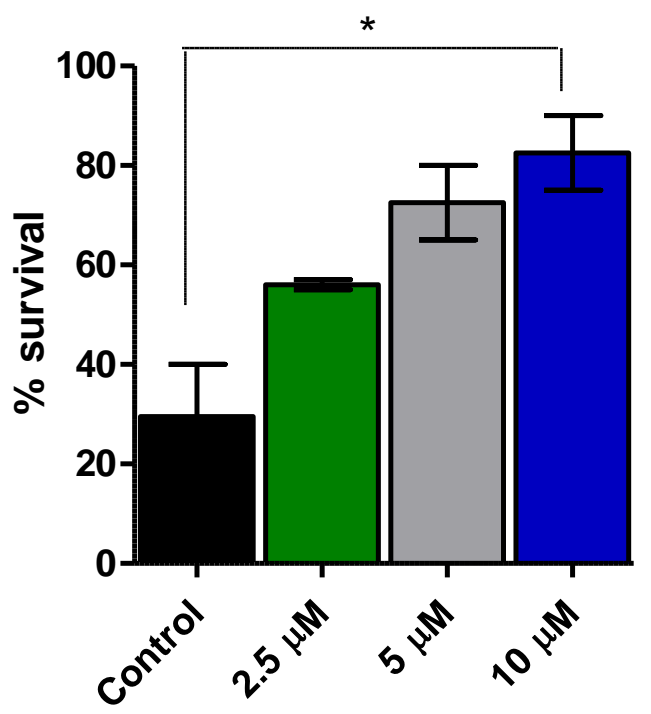

Figure 7.2 Effect of AgiM treatment on the survival of G. Mellonella larvae infected with PA01 strain of $\mathrm{P}$. aeruginosa 
Groups of 10 larvae were infected with 4 x 103 CFU/mL P. aeruginosa PA01 followed by treatment of AgiM (HIM3) administered 20 mins post-infection at concentrations of 2.5 $\mu \mathrm{M}$ (green), $5 \mu \mathrm{M}$ (grey) and $10 \mu \mathrm{M}$ (blue), then incubated at $37^{\circ} \mathrm{C}$ and monitored hourly for survival up to 24-h post-infection. The control group were treated with injections of PBS only (black dotted line). Kaplan-Meier survival curves 7.2a and 7.2b represent two independent experiments using two independent batches of $G$. Mellonella larvae. The bar graph in 7.2c shows the survival proportions of the untreated and treated larvae from the two experiments in 7.2a and 7.2b at 4-h after the onset of larval mortality. Kaplan-Meier $p$ values were calculated using Log Rank (Mantel-Cox) tests to compare each treatment to the untreated control. The bar graph $\mathrm{p}$ values were calculated using a one-way ANOVA with Tukey's post hoc test to compare all treatments to each other and the untreated control. 


\subsubsection{AgiM treatment of $P$. aeruginosa clinical isolates exhibits a dose- dependent increase in larval survival}

The experiment was repeated using the clinical isolates of P. aeruginosa. It was expected that the same trend of dose-dependent enhanced survival would be observed; however, it was unclear if treatment of AgiM would be effective in the highly virulent strain 1079.

The experiment was performed as previously described, using the doses of AgiMs stated in Table 7-1. The effect of AgiM HiM3 treatment on the survival of larvae are shown as Kaplan-Meier survival curves in figure 7.3a-c, and bar graphs representing survival proportion at 4-h post mortality onset in figure 7.4. The results from these experiments show AgiM treatment extends survival in clinical isolates with enhanced survival against strain 1079, moderate enhancement of survival in strain 1205 and poor enhancement of survival in strain 1024 (Figure 7.3a-c).

Analysis of survival curves for larvae infected with the clinical isolates demonstrated that treatment with AgiM HiM3 in a range of concentrations enhanced survival in a dosedependent manner (figure 7.3a,b,c). An increase in survival was observed after AgiM treatment with a significant statistical difference between the untreated control and 10 $\mu \mathrm{M}$ AgiM across all clinical isolates determined by Log Rank (Mantel-Cox) test (at least $\mathrm{p}<0.05$ ) (Figure 7.3a-c),

Larvae infected with strain 1205 shows enhanced survival in all treatment ranges, with only $10 \mu \mathrm{M}$ HiM3 significantly increasing survival to 70-90\% compared to the untreated control which reached $30 \%$ survival $(\mathrm{P}<0.05)$ (figure $7.3 \mathrm{~b}$ ). In contrast, when larvae were infected with strain 1024 all treatment concentrations increased survival; however, it is important to note that the control only achieved $30 \%$ mortality in both repetitions (figure 7.3c). 
Due to variation between experimental repetitions as well as discrepancies in sensitivities between the clinical isolates bar graphs were used to demonstrate survival proportion of larvae at 4-h after onset of mortality (figure $7.4 \mathrm{a}-\mathrm{c}$ ). There was no significant difference between the untreated control and any of the AgiM treatments for strain 1079, which is a highly virulent strain (figure 7.4a). Larvae infected with 1205 and 1024 demonstrated a significant increase in survival after treatment of AgiM in all concentrations compared to the control, determined by one-way ANOVA ( $p<0.05$ )(figure $7.4 \mathrm{~b}-\mathrm{c}$ ). Additionally, there was a significant difference between $2.5 \mu \mathrm{M}$ and 10 $\mu \mathrm{M}$ treatments in 1205 infected larvae $(\mathrm{p}<0.05$ ) (figure $7.4 \mathrm{~b})$.

In summary, these data are consistent with AgiM treatment prolonging survival in a dosedependent manner in larvae infected with P. aeruginosa clinical isolates. 

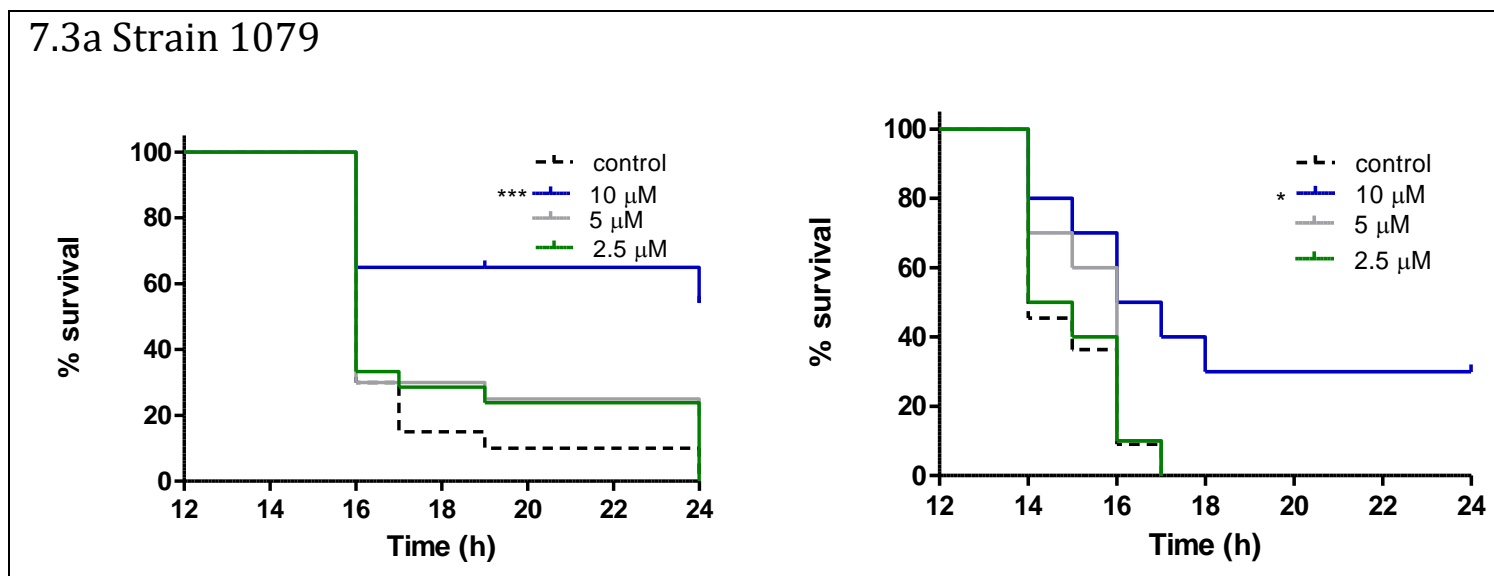

7.3b Strain 1205
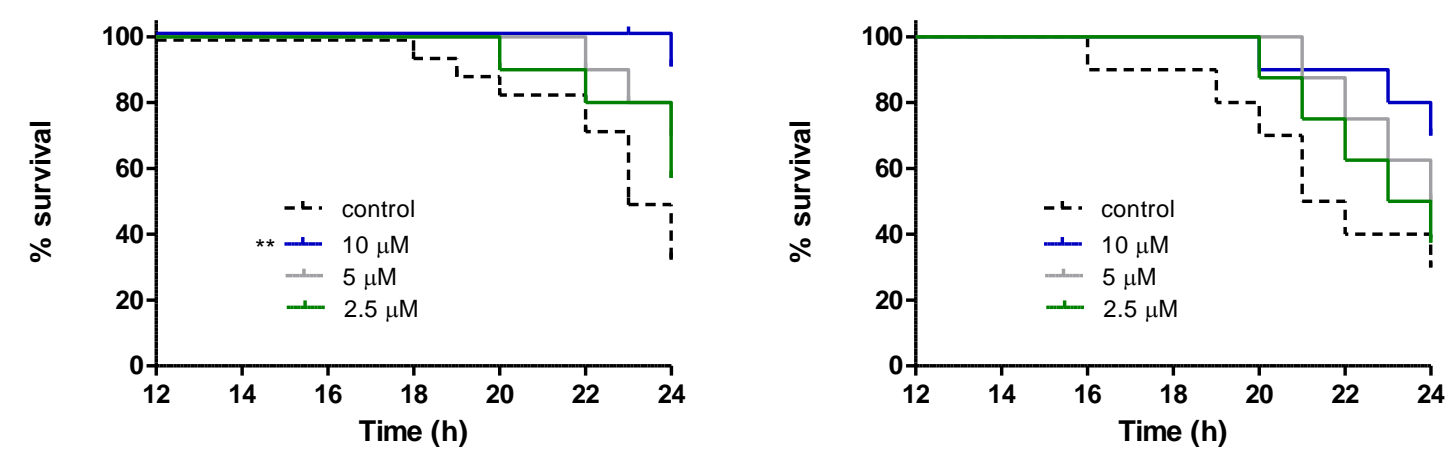

\section{3c Strain 1024}
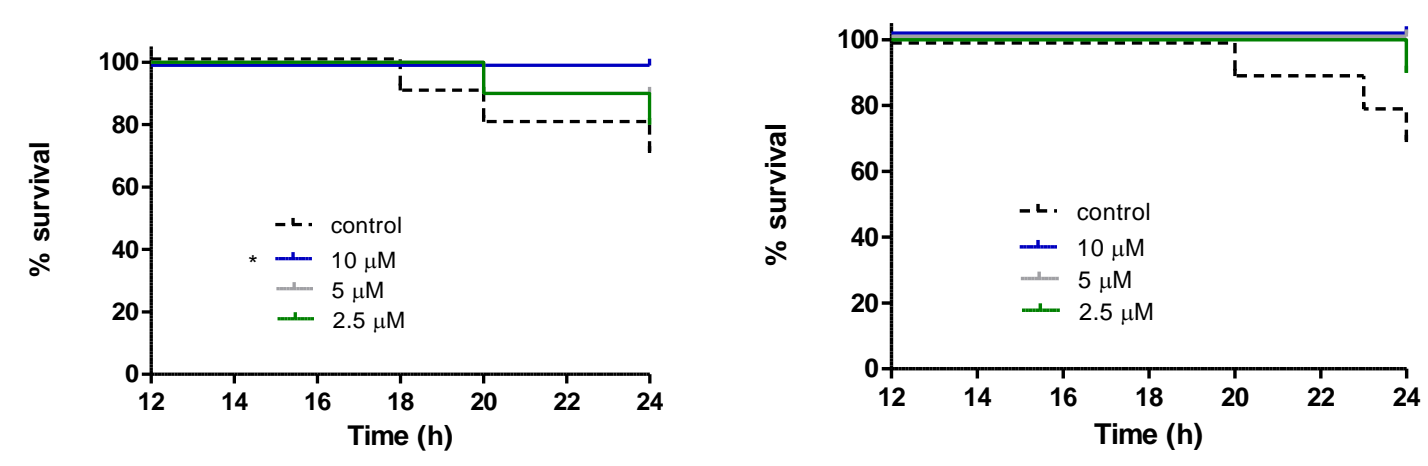

Figure 7.3 Effect of AgiM treatment on the survival of G. Mellonella larvae infected with clinical isolates of $P$. aeruginosa

7.3a-c Larvae were infected with 20 CFU 1079, 100 CFU 1024 and 100 CFU 1205. HiM3 treatments were administered 20-min post-infection at doses of $2.5 \mu \mathrm{M}$ (green), $5 \mu \mathrm{M}$ (grey) and $10 \mu \mathrm{M}$ (blue), and survival monitored hourly for 24-h. Kaplan-Meier survival curves $\mathrm{A}$ and $\mathrm{B}$ represent two independent experiments using two independent batches of $G$. mellonella. P values were calculated using Log Rank (Mantel-Cox) tests to compare each treatment to the untreated control. 
7.4a strain 1079

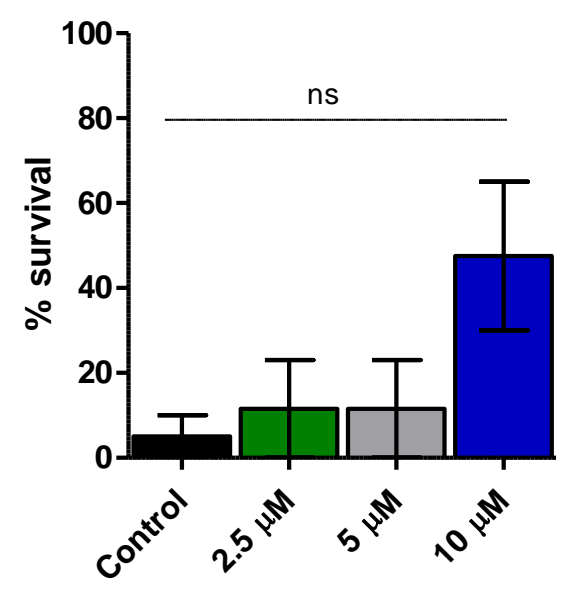

7.4b strain 1205

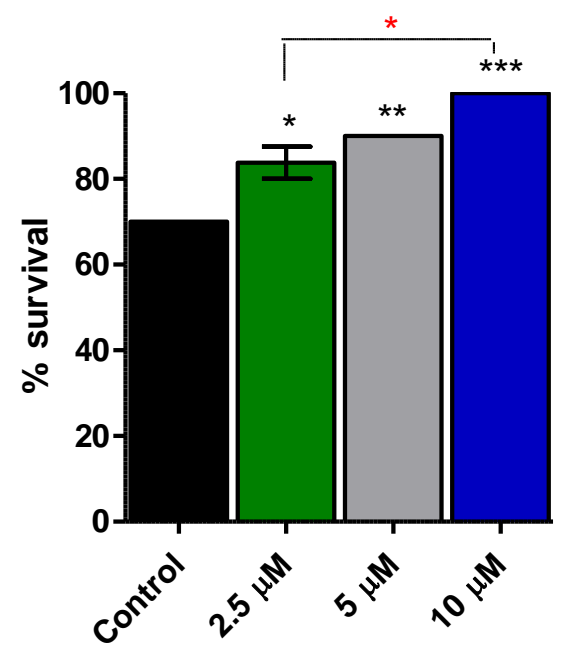

7.4c strain 1024

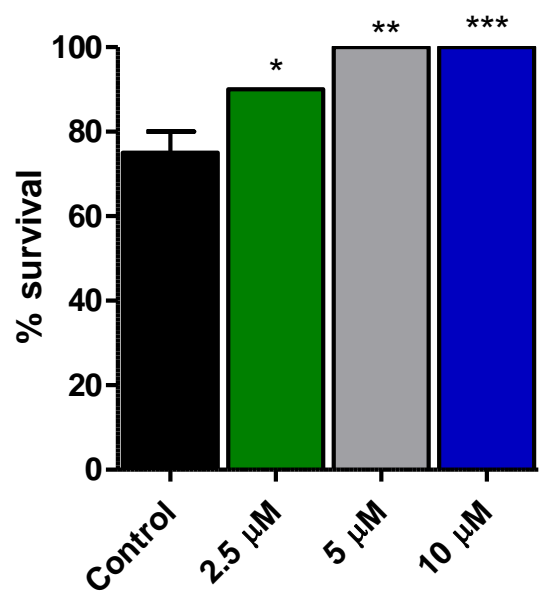

Figure 7.4 Survival proportion of larvae at 4-h after onset of mortality 
7.4a-c. Data from figure 7.3 presented as bar graphs representing the survival percentage of larvae at 4-h post onset of mortality. P values were calculated using a oneway ANOVA with Tukey's post hoc test to compare all treatments to each other and the untreated control. * indicates statistical significance compared to the untreated control, ${ }^{*}$ indicates statistical significance between treatment groups. ${ }^{*}<0.01,{ }^{* *} \mathrm{p}<0.05$, ns $=$ no significance 


\subsubsection{Enhanced larval survival after treatment of AgiM results in a parallel reduction in the number of viable CFU in the larval haemolymph}

An alternative metric to larval survival is the measurement of the bacterial load in the haemolymph of the larvae at different time points after infection. Determination of bacterial load provides insight to the effect of AgiM exposure on the bacteria over the duration of the experiment regardless of larval fate.

\subsubsection{Bacterial load observed at 12 -h and $24-h$ post-infection}

Larvae were infected with $40 \mathrm{CFU} /$ larva of P. aeruginosa strain PA01, then administered a single dose of AgiM (10 $\mu \mathrm{M}) 20$ mins post-infection. After 12-h and 24-h post-infection five larvae from each treatment group were randomly selected and haemolymph extracted and diluted, then plated onto Pseudomonas selection agar. The CFU for each larva was enumerated and plotted (Figure 7.5b-c).

At 12-h post-infection both control and treated larvae exhibited $100 \%$ survival (Figure 7.5a), with comparable internal bacterial loads with a mean of $2.73 \log _{10} \mathrm{CFU} / \mathrm{mL}$ and 2.56 $\log _{10} \mathrm{CFU} / \mathrm{mL}$, respectively (Figure 7.5B).

At 24-h post-infection, the treatment group showed enhanced survival with $40 \%$ mortality, whereas the control group exhibited $100 \%$ mortality (Figure 7.5a). Treatment with $10 \mu \mathrm{M}$ AgiM HiM3 was statistically different from the untreated control $(P=0.0033)$ determined by Log-rank (Mantel-Cox). In parallel, the bacterial load enumerated from the haemolymph correlates with the data, with a mean CFU/mL of $8.85 \log _{10} \mathrm{CFU} / \mathrm{mL}$ for control and 4.09 $\log _{10} \mathrm{CFU} / \mathrm{mL}$ for treatment $(\mathrm{p}<0.001)$ (Figure 7.5B, C).

In summary, at 12 -h post-infection, there is no death in either group, and both show similar bacterial loads. By 24 -h post-infection, the treatment has extended survival and reduced internal bacterial growth. The therapeutic benefit observed from the 
administration of AgiMs correlated with a significantly reduced bacterial load in the haemolymph. 
a

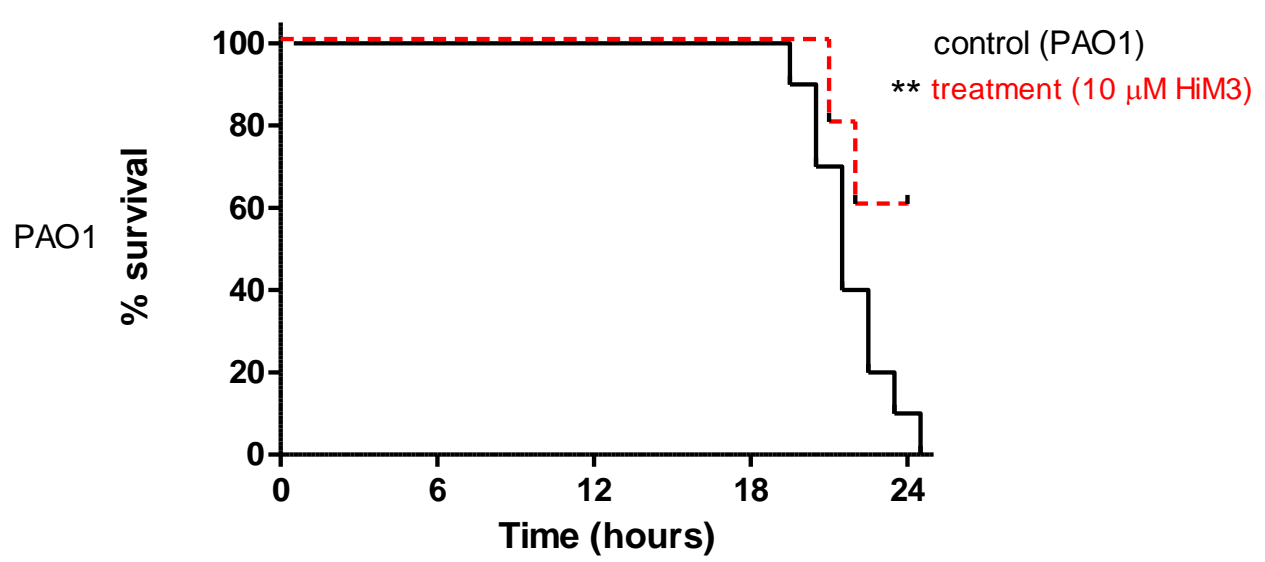

b

c
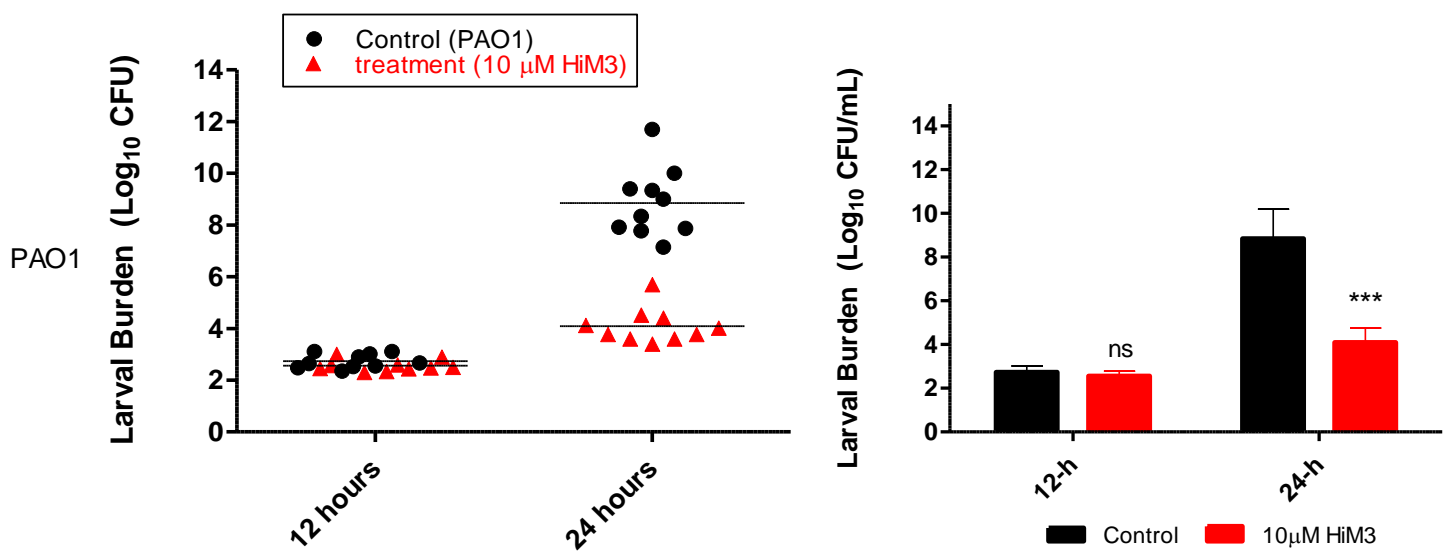

Figure 7.5 The effect of AgiMs treatment on survival of Galleria mellonella and the corresponding internal bacterial load of PA01

7.5a-c: Groups of 10 larvae were infected with $4 \times 10^{3} \mathrm{CFU} / \mathrm{mL}$ of P. aeruginosa strain PA01. A single dose of AgiM HiM3 $(10 \mu \mathrm{M})$ was administered 20 mins post-infection, then larvae incubated at $37^{\circ} \mathrm{C}$. Five larvae from each treatment group were sampled at time points 12 -h and 24-h, and viable internal bacterial load determined by extraction of haemolymph. The haemolymph dilution was plated on Pseudomonas selection agar and incubated for 24-h, with enumeration of viable CFU the following day. $n=10$ (pooled from 2 independent experiments). Data from the experiment presented as (a)Kaplan-Meier survival curves. P values calculated by log-rank (Mantel-Cox) tests to indicate a difference between treatment and the control, ${ }^{* *} \mathrm{p}<0.01$. (b) haemolymph dilutions expressed as viable $\mathrm{CFU} / \mathrm{mL}$. (c )Bar graph represent larval burden as mean+SD, with $\mathrm{P}$ value determined by unpaired t-test, ${ }^{* * *}=\mathrm{p}<0.0001$ 


\subsubsection{The number of viable CFU in haemolymph at the onset of mortality}

The experimental parameters were modified to investigate the number of viable cells at the onset of mortality, as the previous experiment sampled the control larvae at $0 \%$ mortality and $100 \%$ mortality. It was unclear at what point bacterial proliferation occurred, with no difference between control and treatment bacterial load at $12-\mathrm{h}$, and a significant difference at 24-h. It was reasoned that enumeration of viable cells in the haemolymph at the onset of mortality, defined as approximately $20 \%$ mortality of the control would be a more useful metric. This metric may also help to alleviate the variability associated with larval batch susceptibility to $P$. aeruginosa infection, with the onset of mortality ranging from 15 - $21 \mathrm{~h}$ post-infection in different strains (Figure 7.6).

The experiment performed as previously described. Larvae were injected with predetermined bacterial inoculum (Table 7-1), then treated with $10 \mu \mathrm{M}$ AgiM HiM3 20 mins post-infection. Larvae survival was monitored hourly, and five larvae from each treatment group were randomly selected for haemolymph extraction at approximately $20 \%$ mortality within the control group.

Larvae in the untreated control infected with PA01, 1079, 1205 and 1024 reached approximately $20 \%$ mortality at 18-h, 16-h, 21-h and 22-h, respectively (Figure 76 a-g). Comparatively, larvae treated with AgiMs at these time points had $100 \%$ survival for all strains except stain 1079, which reached $10 \%$ mortality (Figure 7.6 c-d).

Measurement of the internal bacterial load of PA01, 1205 and 1024 showed treatment with $10 \mu \mathrm{M}$ AgiMs significantly decreased the viable bacterial cells within each larvae when compared to the untreated control $(P<0.001)$ (figure 7.6b,f,h). PA01 control larvae had a mean burden of $8.20 \log _{10} \mathrm{CFU} / \mathrm{mL}$ with a decrease to $3.30 \log _{10} \mathrm{CFU} / \mathrm{mL}$ in treated larvae. 1205 infected larvae had a mean of $7.55 \log _{10} \mathrm{CFU} / \mathrm{mL}$ compared to 2.80 
$\log _{10} \mathrm{CFU} / \mathrm{mL}$ treated larvae. 1024 infected larvae had the lowest larval burden at 5.85 $\log _{10} \mathrm{CFU} / \mathrm{mL}$, with treated larval burden at $2.47 \log _{10} \mathrm{CFU} / \mathrm{mL}$. Comparison of the mean larval burden between control and treatment shows a $2.3-2.7$ - fold log reduction in viable CFU across these three strains (Table 7-2).

The mean bacterial load of larvae infected with 1079 was $11.10 \log _{10}$ CFU, compared to 9.06 $\log _{10}$ CFU for treated larvae (Table 7-2). Treatment with HiM3 did not significantly enhance survival of larvae or reduce the internal burden. Analysis of Kaplan-Meier survival curves shows only larvae infected with 1205 and 1024 had a significant increase in survival at the end of the 24-h experiment, with strains 1079 and PAO1 showing no significant increase in survival $(P<0.05)$.

In summary, the effect of AgiM treatment on the viability of P. aeruginosa can be observed at the onset of mortality, with a strong correlation between enhanced survival and reduction in larval burden.

Table 7-2 The effect of AgiM treatment on the viability of $P$. aeruginosa strains, and the corresponding log reduction in internal larval burden

\begin{tabular}{|c|c|c|c|c|}
\hline $\begin{array}{c}\text { P.aeruginosa } \\
\text { strain }\end{array}$ & $\begin{array}{c}\text { Inoculum } \\
\text { (CFU/larva) }\end{array}$ & $\begin{array}{c}\text { Untreated control } \\
\text { (Log10 CFU/mL) }\end{array}$ & $\begin{array}{c}\text { Treatment 10uM } \\
\text { AgiM } \\
\text { (Log10 CFU/mL) }\end{array}$ & $\begin{array}{c}\text { Log } \\
\text { reduction }\end{array}$ \\
\hline PAO1 & 40 & 8.20 & 3.30 & 2.5 \\
1079 & 20 & 11.22 & 9.06 & 1.2 \\
\cline { 2 - 5 } 1205 & 100 & 7.55 & 2.80 & 2.7 \\
1024 & 100 & 5.86 & 2.50 & 2.3 \\
\hline
\end{tabular}




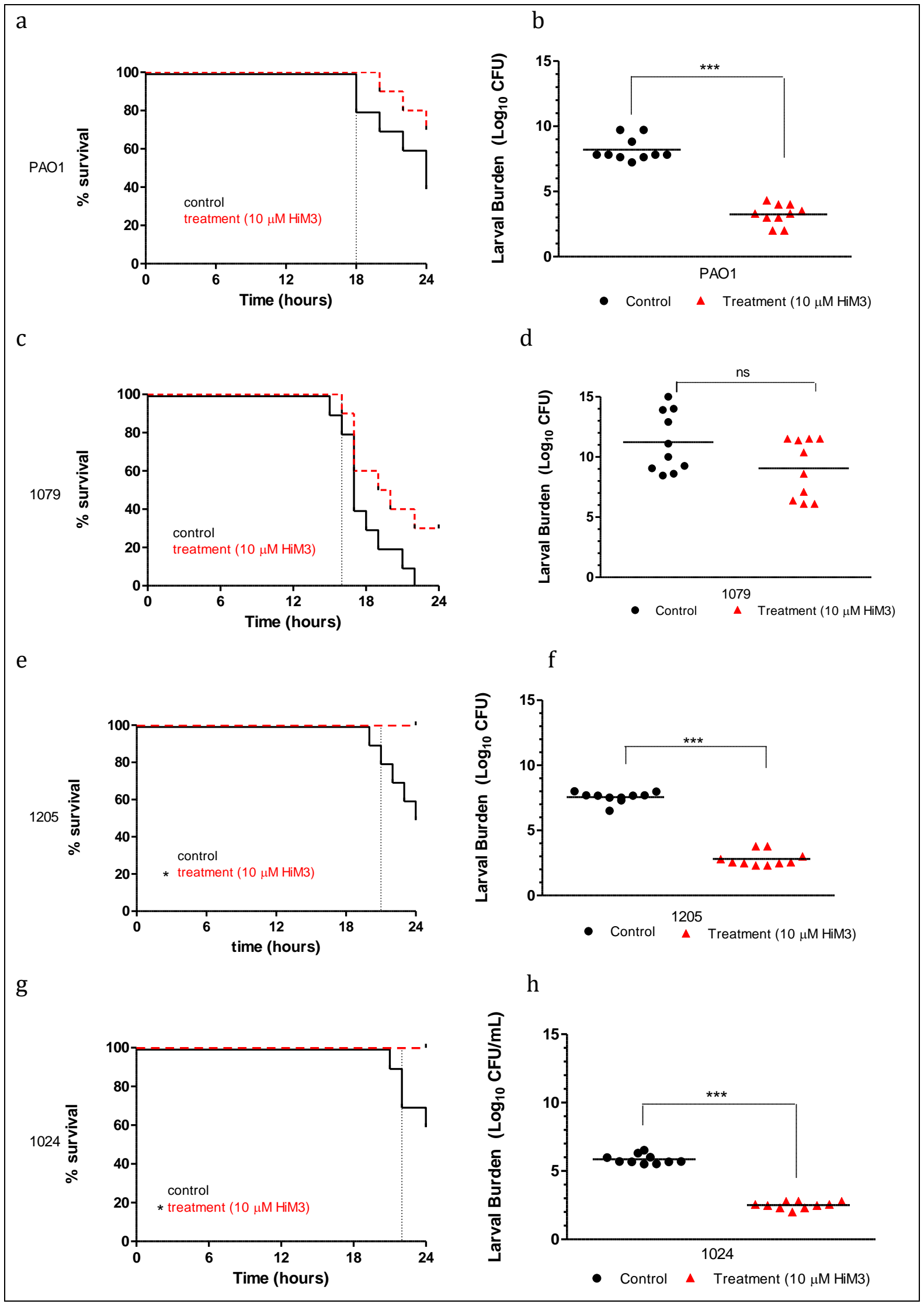

Figure 7.6 The effect of AgiM treatment on survival and internal larval burden of $P$. aeruginosa strains at onset of mortality 
7.6 a-h:-Larvae were infected with strain-specific dose of bacterial suspension and treated with $10 \mu \mathrm{M}$ HiM3 20-mins post-infection (PA01: $4 \times 10^{3} \mathrm{CFU} / \mathrm{mL}, 1079: 2 \times 10^{3}$ $\mathrm{CFU} / \mathrm{mL}, 1205$ and 1024: $10 \times 10^{3} \mathrm{CFU} / \mathrm{mL}$ ). Five larvae from each treatment group were sampled at approximately $20 \%$ mortality in the control of each strain tested, with the haemolymph sampled and dilutions plated on Pseudomonas selection agar. Data presented as (a) Kaplan-Meier survival curves. P values calculated by log-rank (MantelCox) tests to indicate a difference between treatment and the control (b) the larval burden of control and treatment groups as $\log _{10} \mathrm{CFU} / \mathrm{mL}$. P values determined by unparied t-test with Mann-Whitney to compare differences in two groups. 


\subsubsection{AgiMs in combination with tobramycin extend survival of $G$. mellonella}

The previous experiment established the synergistic interaction of AgiMs and tobramycin treatment on P. aeruginosa using checkerboard assays. It was reasoned that combined treatment of AgiMs and tobramycin would enhance survival of G. Mellonella larvae after infection with $P$. aeruginosa.

Experiments were performed as described in Materials and Methods 2.11.1. Larvae were infected with $40 \mathrm{CFU}$ /larva P. aeruginosa PA01 followed by treatment of either AgiMonly, tobramycin-only or AgiM/tobramycin combined and incubated at $37^{\circ} \mathrm{C}$ for 24 -h. Larval survival was monitored hourly and plotted as Kaplan-Meier survival graphs in Figure 7.7.

These data are consistent with AgiMs in combination with tobramycin showing an additive interaction. We were unable to demonstrate a synergistic effect due to the metric of survival.

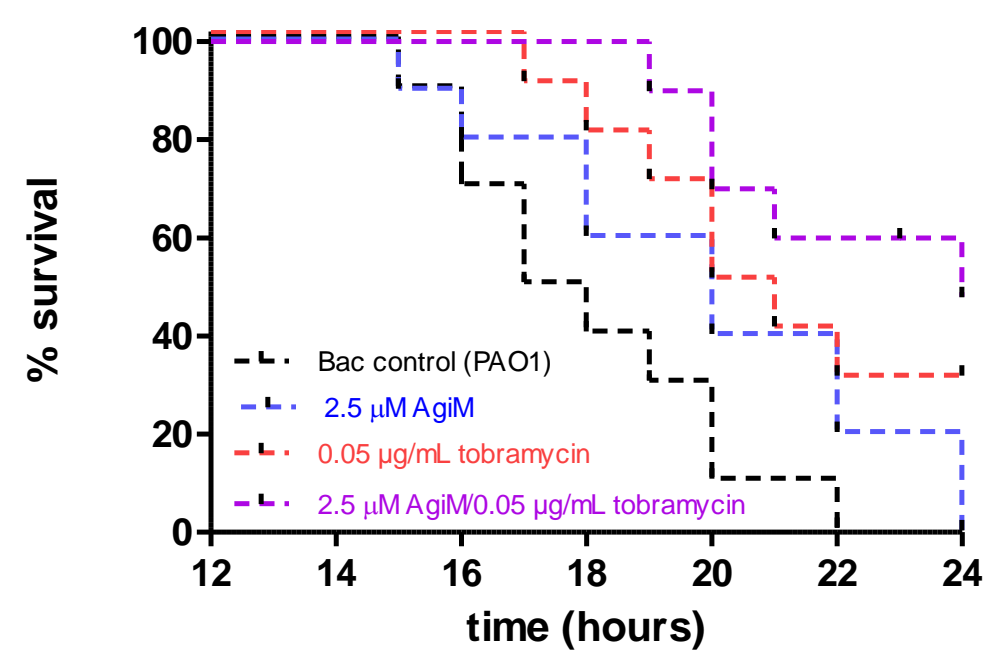

Figure 7.7 Effect of combined treatment of AgiM/tobramycin on the survival of G. Mellonella

Larvae were infected with $40 \mathrm{CFU} /$ larva P. aeruginosa PA01 followed by treatment of either 2.5 $\mu \mathrm{M}$ AgiM-only, $0.05 \mu \mathrm{g} / \mathrm{mL}$ tobramycin-only or $2.5 \mu \mathrm{M} / 0.05 \mu \mathrm{g} / \mathrm{mL}$ AgiM/tobramycin combined and incubated at $37^{\circ} \mathrm{C}$ for 24 -h. 


\subsection{Key findings}

The larvae of $G$. Mellonella provided an adequate host to assess the virulence of $P$. aeruginosa strains used in this study, as well as evaluate the therapeutic efficacy of AgiM HiM3 treatment. Initial experiments to determine the correct bacterial inoculum for each strain highlighted the variety of virulence across different strains of the same bacteria, with 1079 extremely virulent compared to strains 1024 and 1205 . Similar findings of inoculum variation between bacterial strains were described in the literature (89)(93)

The antibacterial activity of AgiMs prolonged the survival of larvae after infection with $P$. aeruginosa. Treatment of AgiM HiM3 in a range of concentrations showed the therapeutic effect was dose-dependent with higher concentrations resulting in enhanced survival. The therapeutic benefit of AgiM observed correlated with the internal bacterial burden inside the larvae. Determination of the larval burden at different time points over the duration of 24-h post-infection with P. aeruginosa showed AgiM treatment at high concentrations $(10 \mu \mathrm{M})$ significantly reduced the number of viable bacteria in the larvae. The effect was observed at the onset of mortality, demonstrating a strong correlation between enhanced survival and reduction in larval burden.

Treatment with AgiM enhanced survival but did not completely rescue larvae from death, rather the survival time was extended. Larvae infected with PA01 had a median survival time of 18-h, with $90 \%$ mortality at 24-h post-infection. Treatment with AgiM extended median survival time by 4 hours (figure $7.2 \mathrm{~b}$ ). These data are consistent with AgiM treatment of larvae infected with $P$. aeruginosa clinical isolates which did not rescue larvae but did extend median survival time by 1-3 hours compared to the untreated control (figure 7.3). A successful therapeutic agent aims to rescue larvae and increase survival to $100 \%$. These data show AgiM at the doses used in this study are not an 
effective treatment but demonstrate the potential for AgiM antibacterial activity. These data show the efficacy of AgiM in vivo correlates with the in vitro sensitivities observed in this study. 


\subsection{Discussion}

The larvae of G. Mellonella provided an adequate model of infection to investigate the effect of AgiMs against P. aeruginosa infections in a physiologically relevant environment. The innate immune system of the larvae involves a cellular and humoral immune response; killing pathogens in a similar process to mammals by employing enzymes such as lysozymes, antimicrobial peptides such as defensins and reactive oxygen species (94). The experiments designed for this chapter were modelled on the literature, with most studies using single treatment doses, usually administered between 20 mins and 2-h after infection of the larvae with the bacteria. In some instances, the treatment was administered immediately after infection (95) or directly prior to infection (96). The administration of AgiM 20-min post-infection was selected for these experiments as the larvae of G. Mellonella are noted to be highly sensitive to infection by P. aeruginosa (89). It was hypothesised that 20 minutes would be sufficient for the infection to take hold followed by systemic delivery of AgiMs directly into the hemocoel of the larvae mimicking the conventional administration route used in mammalian models.

Efforts were made to ensure complete mortality of the control would be achieved within the time frame of the experiment; unfortunately, the high variance observed throughout these experiments made the accurate comparison of survival outcomes difficult, with the control often not reaching $100 \%$ mortality. One of the limitations of using G. Mellonella are the variations between batches in term of susceptibility. A potential solution to this would be large experiments using single batches of larvae, however this was outside the scope of this study. The issue of variation between experimental repetitions was present throughout the experiments in this chapter. Independent experiments, often months apart, used different batches of G. Mellonella, new bacterial inoculums and freshly made 
AgiM. The inconsistencies between replicates could be due to diversity within the $G$. Mellonella batch or colony of $P$. aeruginosa sampled. To minimise the potential for variation within the larval sample population antibiotic-free G. Mellonella larvae were sourced from Biosuppliers, with larvae between 150 - $210 \mathrm{mg}$ selected and starved overnight for experimentation.

Determination of internal bacterial load revealed an unexpected mode of action for AgiMs. The bacterial load of treatment and control were comparable at 12-h postinfection, indicating that treatment had not killed the bacteria or reduced the number of viable cells. At 24-h post-infection there was a visible increase in viable cells in both groups, but a significantly smaller increase in the treatment group compared to the control; 1.59 -fold increase in the treatment group compared to a 3.24-fold increase in the control (Figure 7.5b-c). At the completion of this experiment, the control larvae were all dead, and treatment larvae reached $40 \%$ mortality. It is assumed that if this experiment was continued, the treatment group would reach $100 \%$ mortality as bacterial proliferation exceeded the therapeutic capacity of the single dose of AgiM. Hill and colleagues determined the bacterial load of P. aeruginosa in larvae after treatment with cefotaxime and showed both treatment and control bacterial burdens increased over the course of the experiment, with the treatment group showing significantly reduced growth compared to the control which correlated with prolonged survival. (89)

These findings indicate AgiMs exhibit strong potential as an antibacterial therapeutic. While a single administration of treatment did not completely save the larvae, the results from this chapter show this single dose was capable of significantly reducing the internal bacterial load and extending survival. It is proposed that a second dose of AgiM between the 12-h and 24-h period may have kept the bacterial load suppressed and ensured 100 
$\%$ survival of the larvae. It is interesting to speculate if a second dose would maintain bacterial growth suppression for an extended period to increase survival. It was not possible due to the time constraints of this study to explore this idea. Future investigations should explore the potential therapeutic benefit of repeated-dose on survival. This will be further discussed in the general discussion. 



\section{CHAPTER 8. GENERAL DISCUSSION}

\subsection{Research aims}

The overarching motivation of this thesis was to determine the potential of silver stabilised i-motifs (AgiMs) as a novel therapeutic against Gram-negative bacteria. The rapid increase of chronic Gram-negative infections and the spread of MDR microorganisms are an inescapable reality. The current therapeutic options are quickly depleting with a sharp decrease in new antibiotic development and the isolation of novel compounds from natural sources, necessitating research and development of alternative treatment options. Therefore, this thesis aimed to investigate the antibacterial activity of AgiMs against $P$. aeruginosa, an opportunistic pathogen associated with nosocomial infections displaying a high incidence of intrinsic and acquired resistance mechanisms. The antibacterial potential of AgiMs was assessed in planktonic cultures, biofilm assays and validated in an invertebrate model of infection. Specifically, the aims of this thesis explored the hypothesis that AgiMs deliver soluble $\mathrm{Ag}^{+}$to bacterial cells and offer a novel therapeutic agent for resistant Gram-negative bacteria.

\subsection{Research outcomes}

Overall, the results of this study supported the hypothesis that AgiMs exhibit strong antibacterial activity through the delivery of soluble $\mathrm{Ag}^{+}$. This thesis provides the experimental proof that AgiMs have the potential as an effective therapeutic agent for the treatment of resistant Gram-negative infections.

MIC and IC50 values indicated strong in vitro activity of AgiMs against all strains of studied. Time-kill studies showed that the effect against P. aeruginosa can be bactericidal, 
although this effect was not seen in all strains. The antibacterial activity of AgiMs was demonstrated to be rapid and concentration-dependent, and is comparable to other silver antibacterial agents such as $\mathrm{AgNO}_{3}$ and AgNPs. Current research shows therapeutic potential for AgNPs; these small particles contain a large surface area and exhibit strong antimicrobial activity against various bacteria, viruses and fungi (36). AgNPs contain metallic silver $\mathrm{A}^{0}$, which needs to be converted to the active form of $\mathrm{Ag}^{+}$through oxidative dissolution, notably due to atmospheric $\mathrm{O}_{2}$ (97). In comparison, AgiMs contain ionic silver $\mathrm{Ag}^{+}$, an active antibacterial agent. $\mathrm{Li}$ and colleagues reported that $\mathrm{Ag}^{+}$have a similar mode of action to AgNPs, but exhibit stronger antibacterial activity compared to AgNPs. Administration of silver nitrate $\left(\mathrm{AgNO}_{3}\right)$ was more effective than AgNPs against four bacterial strains, with MICs of $1 \mu \mathrm{g} / \mathrm{mL}$ for $\mathrm{Ag}^{+}$compared to $2-8 \mu \mathrm{g} / \mathrm{mL}$ for AgNPs against P. aeruginosa (98). This reported antibacterial activity of $\mathrm{Ag}^{+}$is consistent with the findings of this thesis, which reported MICs of $0.25 \mu \mathrm{M}$ to $1 \mu \mathrm{M}$ against clinical isolates of P. aeruginosa and MDR strains of $A$. baumannii. Li and colleagues measured antibacterial activity using growth dynamics, reporting silver ions and AgNPs prolonged the growth lag phase in a concentration-dependent manner consistent with the growth profiles seen in this thesis which showed increasing concentrations of AgiMs resulted in a proportional increase in the apparent lag phase (Figure 3.1a-c). AgiM have the additional feature of $\mathrm{Ag}^{+}$ delivery without the corrosive nitrate group associated with $\mathrm{AgNO}_{3}$ application which vastly limits its clinical use.

While the morphological alterations of bacteria after AgiM treatment was not investigated in this thesis, there are several publications indicating $\mathrm{Ag}^{+}$induce severe damage in bacterial cells using TEM (transmission electron microscopy) imaging. These reports show the flagella of bacteria were damaged, restricting movement as well as holes on cell surfaces leading to cytoplasm leakage and ultimately cell death (98)(99). This 
supports $\mathrm{Ag}^{+}$mode of action against bacteria. While antibiotics are designed to target one specific component of the bacterial life cycle, $\mathrm{Ag}^{+}$are not limited to this selectivity and will absorb to any moiety they have a high affinity for, such as thiols, phosphates and amines. This explains the broad-spectrum antibacterial activity of AgiMs in this thesis, with $\mathrm{Ag}^{+}$binding non-specifically to a wide variety of targets and leading to simultaneous perturbation of multiple aspects of cell metabolism, ultimately leading to cell death. AgiMs exhibited rapid killing of $P$. aeruginosa, consistent with published data that indicates $\mathrm{Ag}^{+}$enter bacterial cells within $30 \mathrm{~min}$ of exposition and bind to cytoplasm components, proteins and nucleic acids (49).

The correlation between biofilms and chronic infections is well-established, with $65-80 \%$ of all infections associated with biofilm formation, highlighting their enormous clinical impact (53). It is important to note that while many clinical trials are being conducted with potential antibiofilm agents, there are currently no antibiofilm compounds in clinical use (100). Rather, the current clinical approach is the prevention of biofilm formation. The antimicrobial properties of silver are apparent in these agents, with silver-coated catheters delivering a localised high concentration of $\mathrm{Ag}^{+}$to the areas of potential colonisation and effectively reducing the rate of catheter-related infections. These impregnated devices are effective until the antimicrobial is exhausted, making them ideal for short term implants. Rather than prevention, this thesis investigated the eradication of established biofilms using AgiMs treatment. Treatment of mature P. aeruginosa biofilms indicated a differential response to AgiMs killing effect within the different subpopulations of the biofilm, demonstrated by killing of dispersed cells and disruption of the biofilm biomass but not significantly altering the viability of the remaining cells within the biofilm. This is consistent with the literature, which reports the importance of the silver concentration to kill established biofilms effectively. Bjarnsholt and colleagues 
increased the concentration of silver sulfadiazine to eradicate $P$. aeruginosa biofilms up to 100 -fold compared to that used to eradicate planktonic bacteria, requiring $5-10$ $\mu \mathrm{g} / \mathrm{mL}$ against biofilms compared to $0.156 \mu \mathrm{g} / \mathrm{mL}$ in planktonic culture (79).

It was critical to evaluate the toxicity of AgiMs in an in vivo model. The invertebrate model of infection G. Mellonella was selected, providing an affordable and ethically acceptable model that presents an innate immune response similar to that of mammals (91). This thesis showed a single administration of AgiMs was effective in the extension of survival, with this therapeutic benefit correlated with a reduction in internal bacterial burden. Treatment concentrations up to $10 \mu \mathrm{M}$ (approximately $10 \mathrm{x}$ MIC) remained non-toxic toward the larvae. AgiM treatment was not able to save the larvae, rather extend survival. The potential for synergistic interactions between AgiMs and tobramycin demonstrated in this thesis indicate that combination therapy with AgiMs and antibiotics may enhance the efficacy to be clinically relevant. Recommendations for potential dosing regimes and combination therapy to enhance the therapeutic index of AgiMs are discussed below.

\subsubsection{AgiMs and interval dosing}

As a preliminary study of the antibacterial activity of AgiMs, all experiments were modelled to simulate a single administration of the antibacterial agent after infection. It is hypothesised the single dose of AgiMs is ineffective to treat established P. aeruginosa infections due to decreased permeability into the matrix and low diffusion through the deep layers of the biofilm where the persister subpopulation lies dormant. A proposed strategy to combat these persister cells and facilitate the eradication of biofilms is interval dosing which recommends the administration of multiple doses. The strategy, also called 'pulse dosing' was first proposed in 1944 to eradicate persister cells (85). The regime proposes an initial high dose of the drug to disrupt and kill a proportion of the 
biofilm, then allows the concentration of drug to decrease which signals the release of persister cells and their transition into the planktonic mode of growth. At this point, a second large dose is administered to kill the dispersed cells associated with biofilm recalcitrance. This pulse-dosing can be continued until the eradication of the biofilm is achieved. Figure 8.1 illustrates the proposed effect of interval dosing with AgiM on $P$. aeruginosa biofilms (Figure 8.1). Lewis et al. demonstrated this regime with two consecutive doses of fluoroquinolone to eradicate a P. aeruginosa biofilm in vitro (23). It is was beyond the scope of this thesis to investigate the effect of a multi-dose treatment regime; however, it is recommended that future endeavours explore this strategy. These experiments should focus on 1. the concentration of AgiM, 2. the duration of drug administration, 3. administration in combination with tobramycin and 4. administration of interval dosing

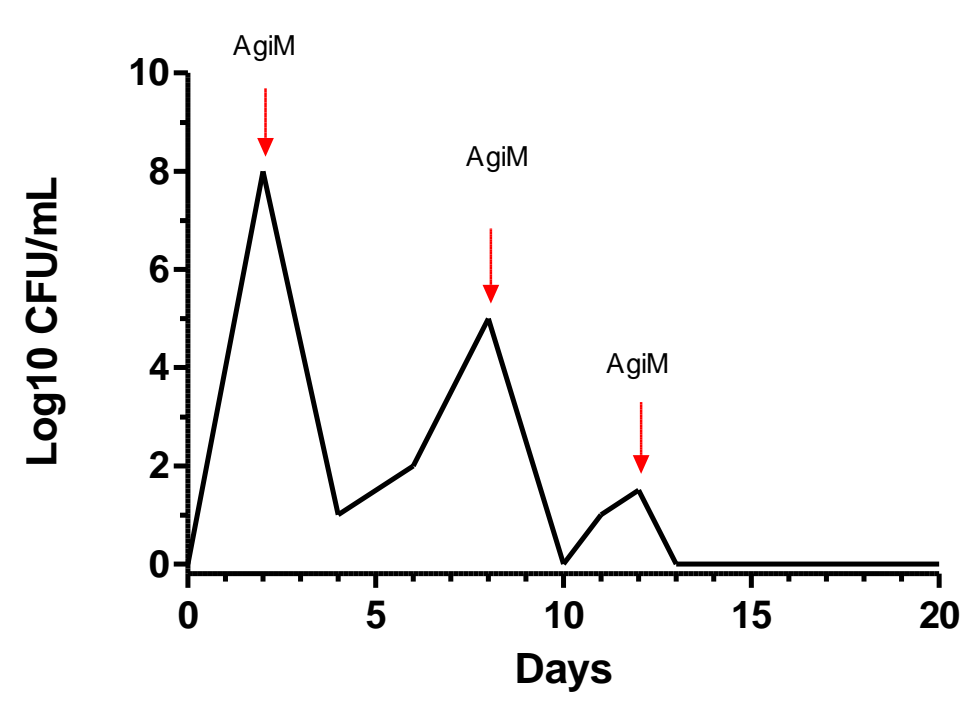

Figure 8.1 Proposed effect of 'interval dosing' of AgiMs on P. aeruginosa mature biofilms

This graph shows the potential mechanism of three administrations of AgiM to first disrupt biofilms, then as the concentration of AgiM lowers and persister cells transition to a planktonic state a second, and if necessary a third dose can be administered to result in the eradication of viable cells in the biofilm. 


\subsubsection{AgiMs and combination therapy}

Multidrug-resistant $P$. aeruginosa strains cause frequent therapeutic failure, with only a limited number of viable antimicrobial options. Multiple studies have demonstrated combination therapy to be effective on P. aeruginosa biofilms in vitro (101)(102)(103) and in animal models of infection (104). The major advantage of combination therapy is that it allows for simultaneous targeting of different bacterial resistance mechanisms, rather than a single antibiotic that is targeted to particular cellular function.

Pamp et al. used a combination of colistin, ciprofloxacin and tetracycline to eradicate $P$. aeruginosa biofilms. It was discovered that ciprofloxacin and tetracycline, which target metabolically active bacteria were effective at the outer layers of the biofilm while in comparison, colistin which targets less active cells was effective at killing the deeper metabolically attenuated inner layers of the biofilm (103). A salient finding from this thesis was the synergistic interaction of AgiMs and tobramycin. It is proposed the Ag+ in AgiM potentiates the activity of tobramycin by targeting multiple metabolic processes inducing oxidative stress and resulting in the damage to the bacterial cell membrane. Silvers ability to permeabilise the outer membrane allows enhanced entry of the aminoglycoside to reach its target and inhibit protein synthesis. These synergistic combinations of sublethal doses of AgiMs and aminoglycosides could restore sensitivity to resistant infections of $P$. aeruginosa, enhancing the currently exhausted antibiotic arsenal. This finding provides the rationale for further exploration of the systemic use of AgiMs in combination with aminoglycosides to treat MDR P. aeruginosa infections.

\subsection{Considerations for AgiMs as a potential therapeutic}

The concentration-dependent antibacterial activity of AgiMs reported in this thesis demonstrate the potential for clinical therapeutic use. The discovery and development of 
new antimicrobial formulations is challenging. The pharmacokinetics of AgiM need to be explored to understand the absorption, distribution, metabolism and elimination of the compound while pharmacodynamics of AgiM would clarify the optimal dose. These parameters are associated with improved clinical outcomes and a reduction in adverse effects (105).

\subsubsection{Resistance}

The prevalence of silver in medical and non-medical antibacterial applications has raised concerns that bacterial resistance will develop compromising its clinical utility, in a manner comparable to the misuse of antibiotics (70). The mechanism of resistance to silver in Gram-negative bacteria involves restricting the accumulation of silver in the periplasm, limiting the amount of silver able to enter the cell. This mode of action can be achieved through endogenous and exogenous resistance mechanisms, inhibiting silver from entering the cell or removing it from the cell, respectively (52). The literature shows that resistance studies indicate selection of silver resistance in Gram-negative bacteria is low. No reduction in silver susceptibility was observed in P. aeruginosa, A. baumannii, $C$. frenundii or $S$. sonnei over 42 days of continuous passage in the presences of $\mathrm{AgNO}_{3}$. Conversely, resistance to silver was selected in E. Coli after only 6 days and was found to be due to upregulation of the copper-related efflux pump cusCFBA (52). Observed selection for resistance seen in vitro in some Gram-negative pathogens suggests the implementation of surveillance programmes to monitor the emergence and spread of silver-resistant strains and to restrict the over-use of silver in non-medical applications could limit the development of resistance and preserve the clinical utility. Silver has been used effectively as an antimicrobial for centuries with very few reports of resistance, indicating the development of resistance is low. However, it would be inadvisable to 
under-estimate the remarkable ability of bacteria to adapt and survive when faced with a challenge.

\subsubsection{Potential silver toxicity}

An important consideration when developing new silver-based therapeutics is the potential for cytotoxicity when used in humans. The National Institute for Occupational Safety and Health recommended exposure limit is $0.01 \mathrm{mg} / \mathrm{m}^{3}$ for all forms of silver; however, there are discrepancies in exposure limits across different regulatory boards with some recommending $0.1 \mathrm{mg} / \mathrm{m}^{3}$ (106). Chronic ingestion or inhalation of silver can lead to the development of argyria, a permanent bluish-grey discolouration of the skin or argyrosis, discolouration of the eyes. Exposure to soluble silver compounds can also produce adverse toxic effects such as liver and kidney damage, irritation of the respiratory and intestinal tract, and changes in blood cells. Clinical exposure of controlled silver concentrations is reported to have minimal risk of toxicity (107), which supports the importance of pharmacokinetics when developing new silver-based therapeutics to ensure $\mathrm{Ag}^{+}$absorption in the body remains below cytotoxic thresholds. This was not explored for AgiM in this thesis, but future experiments will need to determine the quantity of silver and the stability and dissolution rate of $\mathrm{Ag}^{+}$to ensure silver levels below the cytotoxic threshold are maintained. This will be discussed further in Future directions 8.6.

The ecological impact of the wide-spread use silver-based antibacterial agents is a rising concern and needs to be considered when designing silver-based therapeutics. Once released back into the environment, silver may present adverse effects on bacteria, algae, invertebrates and vertebrates (45). To remedy this, it is essential to understand the mechanisms of silver ions in the environment clearly and to establish systems to 
minimise their environmental impact. There are currently ongoing studies to determine the impact of AgNPs on wastewater treatment plants and if chemical treatment will inactivate them (106).

\subsubsection{Route of administration}

The route of administration for AgiMs requires careful consideration to ensure therapeutically effective drug concentrations with high efficacy and reduced toxicity. Standard antimicrobial drugs are most commonly delivered systemically by oral or parental administration in the form of tablets and injections. This method exposes the body to high concentrations of the drug, rather than just the site of infection, which confers a high risk of adverse effects and potential systemic toxicity. A major disadvantage of this method is first-pass elimination, whereby the concentration of a drug is greatly reduced due to significant metabolism in the liver or gastrointestinal tract before it reaches systemic circulation (108).

Systemic administration is not advised for DNA-based therapeutics such as AgiMs due to the rapid degradation of DNA by endogenous nucleases and low absorption through the gastrointestinal tract. However, chemical modifications to DNA-based compounds can restore the in vivo capabilities of DNA-based compounds, such as 3'-end capping with inverted thymidine to protect against 3'-exonuclease in human serum (97). The main concern for the systemic delivery of AgiMs is related to $\mathrm{Ag}^{+}$cytotoxicity and inactivation of $\mathrm{Ag}^{+}$in serum. The accumulation of $\mathrm{Ag}^{+}$in major organs is a serious concern after a study by Guo and colleagues reported organ toxicity in kidneys, lungs and liver after intravenous injection of AgNPs in mice (109).

Silver-based antimicrobials are most commonly applied topically, via a soaking solution (AgNO3), cream (silver sulfadiazine) or an impregnated dressing (silver-coated 
dressings). Topical administration is an advised method of delivery for AgiMs, in order to avoid the many unwanted issues of systemic administration. Another promising method of delivery is parental administration to pulmonary airways. This non-invasive delivery system localises the drug to the lungs and avoids metabolism in the liver. In 1997 the FDA approved tobramycin designed to be inhaled for treatment of chronic $P$. aeruginosa infections in CF patients (86). Aerosol delivery for inhaled antibiotics maximises drug delivery to the airways while limiting the potential systemic side effects (110). Administration of AgiMs directly to the pulmonary airways requires extensive investigation to elucidate the potential toxicity. Studies on the application of AgNPs in the lungs of rats demonstrated transient lung inflammation and the beginning of genotoxic effects, with focal deposition of silver in lymph nodes, liver, spleen and kidney observed by LA-ICP-MS (Laser Ablation Inductively Coupled Plasma Mass Spectrometry) (111). The potential applications of AgiMs are discussed in section 8.4

\subsubsection{Stabilisation of i-Motif DNA}

Silver stabilised i-motifs are inherently stable at neutral $\mathrm{pH}$; however, there are many reported modifications to i-motifs that importantly do no alter the conformational structure. Maintaining the integrity of the DNA i-Motif structure is crucial to prevent destabilisation or unfolding into ssDNA. Several chemical modifications of these structures have been reported to increase stability whilst maintaining conformational integrity. These modifications include, but are not limited to; fluorine substitutions (57), phenoxazine 2'-deoxynucleotide (i-clamp) substitutions (112), LNA incorporation (113) and PNA nucleic modification (63). Fluorine substitution at the sugar moiety by 2-deoxy2-fluoroarabinose lead to significant stabilisation of i-Motif in a wide range of $\mathrm{pH}$ conditions. Substitution of i-clamp leads to thermal stabilisation in a range of $\mathrm{pH}$

conditions without compromising base-pair interactions and conformation. Another 
study confirmed C-rich peptide nucleic acid (PNA) could form i-Motifs. PNA, a synthetic analogue of DNA, offers the structural features of DNA i-Motifs without the negatively charged sugar backbone. Locked nucleic acid (LNA) is a conformational locked RNA that can increase the thermal stability of i-Motifs. These modifications increase the stability of i-Motifs for in vitro and in vivo studies as well as potential future clinical applications.

\subsubsection{The commercial viability of AgiM}

The cost of commercial synthesis of AgiMs is an important consideration to establish the viability for clinical use. The financial burden of large scale DNA synthesis could be a limiting factor in AgiM development. Therefore the length of i-motif sequences selected are important to consider when evaluating the potential cost of synthesis; indicating the smaller half i-motif sequences are more attractive commercially than the larger full imotif sequences. Conversely, exploration of alternative synthesis methods for AgiM could reduce the high cost of large scale commercial synthesis. Rolling circle amplification (RCA) is a potential method; it utilises an isothermic enzymatic process to rapidly generate long ssDNA using a circular DNA template and special DNA polymerases (114)(115). A simple and versatile technique, RCA allows products to be specifically designed by modification of the circular template. This technique has been used extensively to construct nanostructures and multivalent molecular scaffolds for various biomedical applications, including diagnostics and therapeutics (114).

\subsection{Potential applications of AgiMs}

\subsubsection{Burn wound care management}

Topical applications of AgiMs in a cream or solution for the management of burn wounds holds promise. Unfortunately, the current commercial market is already saturated with a vast array of silver-based antibacterial products, including but not limited to AgSD, 
$\mathrm{AgNO}_{3}$, silver-coated dressings and medical devices. The predicted cost of commercial production of AgiMs for this application makes it an unviable option. The application of AgiMs as a therapeutic is better suited to more chronic disorders with inadequate strategies available, as discussed below.

\subsubsection{Diabetic foot ulcers}

Diabetic foot ulcers are a major complication associated with patients with diabetes mellitus. Individuals suffering from this chronic disorder are at high risk of developing microbial infections, with foot ulcers the leading cause of hospitalisation and amputation of lower limbs (116). The challenge of wound management for diabetic patients is confounded by the reduced blood circulation at the extremities, which makes systemic administration of treatment mostly ineffective. The current literature shows treatment with silver as a potential strategy. Hernandez and colleagues demonstrated topical administration of AgNPs (1.8 mg/mL) every 24-h for 7 days resulted in significant improvement and closure of the wound in diabetic patients (117). Therefore, it indicates the topical administration of AgiMs as a potent antibacterial agent to treat these diabetic ulcers is a viable option. The application of AgiMs in a cream formation could allow for improved and prolonged delivery of $\mathrm{Ag}^{+}$directly to the wound compared to liquid solutions. Comparison of AgiMs to other silver-based antimicrobials available shows AgiM has the advantage of delivering soluble $\mathrm{Ag}^{+}$directly to the site of infection. The combination of AgiM with a broad-spectrum antibiotic such as ciprofloxacin could aid in the rapid eradication of these chronic infections.

\subsubsection{Cystic fibrosis and respiratory tract infections}

Cystic fibrosis (CF) is a genetic disease characterised by a mutation in the cystic fibrosis transmembrane regulator (CFTR), resulting in abnormal airway surface liquid (ASL) that 
hinders regular mucociliary clearance from the lungs (118). The altered airway epithelia encourage inflammation while impairing the immune response during infections promoting chronic colonisation of the lungs by P. aeruginosa leading to pulmonary failure and is the cause of death in over $90 \%$ of patients. Bacterial infections of the lungs occur at a young age in $\mathrm{CF}$, with up to $85 \%$ of patients with chronic $P$. aeruginosa infections by adolescence (118).

The delivery of tobramycin by inhalation is a well-established treatment for Pseudomonas aeruginosa infections in patients with CF (118) and is also used to treat non-CF patients with respiratory tract disease (110). Nebulised antimicrobials allow deposition of the drug in the lungs at much higher concentrations than those that can be achieved through intravenous administration and avoids the systemic exposure and toxicity associated with that delivery. Current treatment regimes temporarily improve pulmonary function; however, eradication of chronic infection is rare. This in part, is due to antibiotic resistance and the inadequate disruption of biofilms and targeting of persistor cells within them.

More effective therapeutic strategies are needed. The synergistic combination of AgiMs with tobramycin is a potential treatment for these chronic P. aeruginosa infections. However, further investigation into the antibacterial activity of AgiMs and tobramycin and the ability to disrupt biofilm formation is required. The hypothesis is that AgiMs and tobramycin in synergy would be capable of disrupting biofilms and allow access to the persistor cell population beneath. The synergistic activity enhances the therapeutic index of tobramycin, allowing the use of a lower dose of antibiotic. Silver has the advantage of targeting dormant persistor cells as its mechanism is independent of bacterial metabolism, unlike many antibiotics. There is precedence for silver to treat respiratory 
infections in the literature. Cannon and colleagues found silver coupled to a methylated caffeine carrier displayed in vitro efficacy against drug-resistant $P$. aeruginosa isolated from patients with respiratory tract infections. The therapeutic efficacy of this compound was validated in a murine model of severe lung sepsis, showing mice in the treatment group had increased survival with decreased levels of bacteria in the lung (107). These findings support the further exploration of AgiM as a potential therapy for bacterial respiratory tract infections.

\subsection{Limitations}

The results obtained in this thesis need to be considered in the light of experimental limitations. Unfortunately, time constraints prohibited the quantification of bound $\mathrm{Ag}^{+}$in AgiM structures, which could be used to validate the antibacterial activity reported in this thesis. Therefore, to further optimise AgiMs for clinical efficacy, it is essential to undertake pharmacokinetic and pharmacodynamic studies which will be further discussed in Future Direction 8.6.

In addition, this thesis predominately used P. aeruginosa as the selected pathogen with only preliminary data in A. baumannii. These two priority pathogens were selected due to their high incidence of resistance and the urgent need for novel therapeutics. Evaluation of antibiotic susceptibilities of clinical isolates was undertaken near the completion of this thesis which revealed no MDR resistant strains of $P$. aeruginosa were used. Strain variations between the isolates allowed observation of AgiMs ability to interact with different levels of virulence; however, validation in MDR strains is vital due to the mutagenic nature of P. aeruginosa and global increase in resistant strains. It would be pertinent for future experiments to continue the investigation of AgiMs against $A$. 
baumannii and other Gram-negative and Gram-positive pathogens to establish how broad the antibacterial potential of AgiMs are.

A significant obstacle in antimicrobial susceptibility testing is the correlation between in vitro data into in vivo efficacies. Discrepancies in therapeutic relevance hinder researchers in the development of antimicrobials. Common in vitro susceptibility testing methods; agar diffusion, agar dilution and broth dilution can be used to detect MIC values but importantly do not provide a comparable environment to the human body. However, regardless of inherent limitations of in vitro susceptibly testing, it is an invaluable tool for scientists to evaluate antimicrobial susceptibilities and resistance in a time and costefficient manner. A long-established practice, the use of animals in susceptibility testing is an essential prerequisite to clinical development (104). The use of animal models is ethically contentious and can be an expensive and laborious task for researchers to undertake. The purpose of in vivo testing is to place the results of in vitro testing in the context of clinical relevance to humans. These models of infection offer insight into antimicrobial activity in the host environment, giving valuable information on efficacy and toxicity. Discrepancies between results of in vitro and in vivo antimicrobial susceptibly testing is a common hindrance when evaluating the clinical importance of antimicrobial agents (104). Despite this, both methods are necessary to advance potential novel therapeutics to clinical trials.

\subsection{Future directions}

This thesis provides an investigation of AgiMs antibacterial activity against $P$. aeruginosa, assessing its ability using both in vitro and in vivo models of infection. The results 
obtained from this thesis indicate a more substantial examination of AgiMs is warranted, with the overarching aim of progressing to animal models and clinical trials.

AgiMs exhibited strong antibacterial activity in clinical isolates of $P$. aeruginosa and resistant strains of $A$. baumannii. This indicates other Gram-negative bacteria would also be susceptible to AgiM treatment. It would be worth considering the effect of AgiMs against the ESKAPE pathogens, a collection of the common pathogens demonstrating high levels of pathogenesis and resistance. The strong antibacterial activity reported in this thesis has led to a new project investigating the distribution of $\mathrm{Ag}^{+}$into remote organs of rats secondary to the application of AgiMs through intravenous administration. Weimann and colleagues carried out a similar study on AgNPs, which reported focal accumulations of $\mathrm{Ag}^{+}$in peripheral organs (111). This new project will also aim to elucidate the silver content of AgiMs using atomic spectroscopy.

Inhalation of AgiMs is potentially one of the most important routes of exposure that requires careful consideration of the potential health hazards and will hopefully be explored in the future. Nebulised antibiotics are commonly administered for chronic $P$. aeruginosa infections of CF patients, and the addition of AgiMs in a synergistic combination could be a potential therapeutic option. Particle pharmacokinetics combined with studies of dissolution rates would provide insights into the impact of in vivo AgiM administration. Previous experiments by Takenaka and colleagues reported the inhalation of $15 \mathrm{~nm}$ AgNPs in rats showed a rapid decrease in lung particle content directly following inhalation with subsequent translocation to the blood circulation and distribution to the liver, kidney and brain (119). These findings show the importance of a clear understanding of AgiMs in vivo bioreactivity to allow the development of a safe and effective therapeutic agent. 


\subsection{Conclusions}

This thesis aimed to assess the efficacy of AgiMs for the treatment of resistant Gramnegative infections. The antibacterial activity of AgiMs was assessed in planktonic cultures of $P$. aeruginosa and $A$. baumannii, two common opportunistic pathogens associated with high morbidity and mortality rates globally. AgiMs exhibited strong concentration-dependent antibacterial activity and synergistic interactions with aminoglycosides. The antibacterial activity of AgiM treatment was validated against mature P. aeruginosa biofilms, with low concentrations of AgiMs disrupting and reducing the mass of established biofilms; however, these concentrations were unable to eradicate the biofilms. The in vitro findings were mirrored in vivo in an invertebrate model of infection, which demonstrated treatment extended the survival of larvae. The therapeutic benefit of AgiMs correlated with the reduction in the internal bacterial load of the larvae. Altogether these results indicate a strong antibacterial activity against resistant Gramnegative bacteria, but that AgiMs alone are not sufficient to be clinically relevant at present.

These findings support the recommendation of continued research into AgiMs as an antibacterial to treat chronic diseases with inadequate treatment options such as diabetic ulcers and respiratory tract infections prevalent in CF patients. Future studies should be conducted to improve the efficacy of AgiMs. First, the quantification of silver content and elucidation of the optimal poly-C tract length and turn sequence for antibacterial efficacy, biosafety and biocompatibility to attain increased therapeutic benefits and reduced potential side effects. Secondly, an examination of the cytotoxicity, genotoxicity, and inflammatory response of the AgiMs toward human cells and murine models of infection. 
Altogether, the findings of this thesis provide a preliminary evaluation of the antibacterial of AgiMs and support the continued exploration of its therapeutic potential against the increasing threat of resistant pathogens. 


\section{BIBLIOGRAPHY}

1. Davies J, Davies D. Origins and Evolution of Antibiotic Resistance. Microbiol Mol Biol Rev [Internet]. 2010;74(3):417-33. Available from:

http://mmbr.asm.org/cgi/doi/10.1128/MMBR.00016-10

2. Rice LB. Federal Funding for the Study of Antimicrobial Resistance in Nosocomial Pathogens: No ESKAPE. J Infect Dis. 2008;197(8):1079-81.

3. Tanwar J, Das S, Fatima Z, Hameed S. Multidrug resistance: An emerging crisis. Interdiscip Perspect Infect Dis. 2014;2014.

4. Feng Y, Hodiamont CJ, Van Hest RM, Brul S, Schultsz C, Ter Kuile BH. Development of antibiotic resistance during simulated treatment of Pseudomonas aeruginosa in chemostats. PLoS One. 2016;11(2):1-12.

5. Breidenstein EBM, de la Fuente-Núñez C, Hancock REW. Pseudomonas aeruginosa: All roads lead to resistance. Trends Microbiol. 2011;19(8):419-26.

6. Mathee K, Ciofu O, Sternberg C, Lindum PW, Campbell JIA, Jensen P, et al. Mucoid conversion of Pseudomonas aeruginosa by hydrogen peroxide: A mechanism for virulence activation in the cystic fibrosis lung. Microbiology. 1999;145(6):134957.

7. Magana M, Sereti C, Ioannidis A, Mitchell CA, Ball AR, Magiorkinis E, et al. Options and limitations in clinical investigation of bacterial biofilms. Clin Microbiol Rev. 2018;31(3):1-49.

8. Blair JMA, Webber MA, Baylay AJ, Ogbolu DO, Piddock LJV. Molecular mechanisms 
of antibiotic resistance. Nat Rev Microbiol. 2015;13(1):42-51.

9. Hancock REW. Resistance mechanisms in Pseudomonas aeruginosa and other nonfermentative gram-negative bacteria. Clin Infect Dis. 1998;27(SUPPL.1):93-9.

10. Hirakata Y, Srikumar R, Poole K, Gotoh N, Suematsu T, Kohno S, et al. Multidrug efflux systems play an important role in the invasiveness of Pseudomonas aeruginosa. J Exp Med. 2002;196(1):109-18.

11. Ohene-Agyei T, Lea JD, Venter H. Mutations in MexB that affect the efflux of antibiotics with cytoplasmic targets. FEMS Microbiol Lett. 2012;333(1):20-7.

12. Munita JM, Arias CA. Mechanisms of Antibiotic Resistance.

13. Obritsch MD, Fish DN, MacLaren R, Jung R. Nosocomial infections due to multidrug-resistant Pseudomonas aeruginosa: Epidemiology and treatment options. Pharmacotherapy. 2005;25(10 I):1353-64.

14. Hahn A, Burrell A, Fanous H, Chaney H, Sami I, Perez GF, et al. Antibiotic multidrug resistance in the cystic fibrosis airway microbiome is associated with decreased diversity. Heliyon [Internet]. 2018;4(9):e00795. Available from: https://doi.org/10.1016/j.heliyon.2018.e00795

15. Exner M, Bhattacharya S, Christiansen B. Antibiotic resistance: What is so special about multidrug-resistant Gram-negative bacteria? multiresistenten Bakterien? [Internet]. [cited 2019 Jun 27]. Available from: https://www.ncbi.nlm.nih.gov/pmc/articles/PMC5388835/pdf/HIC-12-05.pdf

16. Mulcahy LR, Isabella VM, Lewis K. Pseudomonas aeruginosa Biofilms in Disease. Microb Ecol. 2014;68(1):1-12. 
17. Joo HS, Otto M. Molecular basis of in vivo biofilm formation by bacterial pathogens. Chem Biol [Internet]. 2012;19(12):1503-13. Available from: http://dx.doi.org/10.1016/j.chembiol.2012.10.022

18. Lebeaux D, Ghigo J-M, Beloin C. Biofilm-Related Infections: Bridging the Gap between Clinical Management and Fundamental Aspects of Recalcitrance toward Antibiotics. Microbiol Mol Biol Rev. 2014;78(3):510-43.

19. Mah TFC, O'Toole GA. Mechanisms of biofilm resistance to antimicrobial agents. Trends Microbiol. 2001;9(1):34-9.

20. Maurice NM, Bedi B, Sadikot RT. Pseudomonas aeruginosa biofilms: Host response and clinical implications in lung infections. Am J Respir Cell Mol Biol. 2018;58(4):428-39.

21. Petrova OE, Sauer K. Escaping the biofilm in more than one way: Desorption, detachment or dispersion. Curr Opin Microbiol [Internet]. 2016;30:67-78. Available from: http://dx.doi.org/10.1016/j.mib.2016.01.004

22. Moradali MF, Ghods S, Rehm BHA. Pseudomonas aeruginosa lifestyle: A paradigm for adaptation, survival, and persistence. Front Cell Infect Microbiol. 2017;7(FEB).

23. Lewis K. Persister cells, dormancy and infectious disease. Nat Rev Microbiol. 2007;5(1):48-56.

24. Wood TK, Knabel SJ, Kwan BW. Bacterial persister cell formation and dormancy. Appl Environ Microbiol. 2013;79(23):7116-21.

25. Kang D, Revtovich A V., Chen Q, Shah KN, Cannon CL, Kirienko N V. PyoverdineDependent Virulence of Pseudomonas aeruginosa Isolates From Cystic Fibrosis Patients. Front Microbiol. 2019;10(September):1-12. 
26. Nichols WW, Dorrington SM, Slack MPE, Walmsley HL. Inhibition of tobramycin diffusion by binding to alginate. Antimicrob Agents Chemother. 1988;32(4):51823.

27. Azeredo J, Azevedo NF, Briandet R, Cerca N, Coenye T, Costa AR, et al. Critical review on biofilm methods. Crit Rev Microbiol. 2017;43(3):313-51.

28. Kirienko DR, Kang D, Kirienko N V. Novel pyoverdine inhibitors mitigate Pseudomonas aeruginosa pathogenesis. Front Microbiol. 2019;10(JAN):1-14.

29. Poole K. Pseudomonas aeruginosa: Resistance to the max. Front Microbiol. 2011;2(APR).

30. Gibson GA, Bauer SR, Neuner EA, Bass SN, Lam SW. Influence of colistin dose on global cure in patients with bacteremia due to carbapenem-resistant gramnegative bacilli. Antimicrob Agents Chemother. 2016;60(1):431-6.

31. Wang $\mathrm{L}, \mathrm{Hu} \mathrm{C}$, Shao L. The antimicrobial activity of nanoparticles: Present situation and prospects for the future. Int J Nanomedicine. 2017;12:1227-49.

32. Podolsky SH. The evolving response to antibiotic resistance (1945-2018). Palgrave Commun [Internet]. 2018;4(1). Available from: http://dx.doi.org/10.1057/s41599-018-0181-x

33. Scoffone VC, Trespidi G, Chiarelli LR, Barbieri G, Buroni S. Quorum sensing as antivirulence target in cystic fibrosis pathogens. Int J Mol Sci. 2019;20(8).

34. Gellatly SL, Hancock REW. Pseudomonas aeruginosa: New insights into pathogenesis and host defenses. Pathog Dis. 2013;67(3):159-73.

35. Morones JR, Elechiguerra JL, Camacho A, Holt K, Kouri JB, Ramírez JT, et al. The 
bactericidal effect of silver nanoparticles. Nanotechnology. 2005;16(10):2346-53.

36. Möhler JS, Sim W, Blaskovich MAT, Cooper MA, Ziora ZM. Silver bullets: A new lustre on an old antimicrobial agent. Biotechnol Adv. 2018;36(5):1391-411.

37. Alexander JW. History of the Medical Use of Silver. Surg Infect (Larchmt) [Internet]. 2009;10(3):289-92. Available from:

http://www.liebertonline.com/doi/abs/10.1089/sur.2008.9941

38. Klasen HJ. A historical review of the use of silver in the treatment of burns. II. Renewed interest for silver. Burns. 2000;26(2):131-8.

39. Maillard JY, Hartemann P. Silver as an antimicrobial: facts and gaps in knowledge. Crit Rev Microbiol. 2013;39(4):373-83.

40. Barras F, Aussel L, Ezraty B. Silver and antibiotic, new facts to an old story. Antibiotics. 2018;7(3):1-10.

41. Blaskovich MAT, Butler MS, Cooper MA. Polishing the tarnished silver bullet: the quest for new antibiotics. Essays Biochem [Internet]. 2017;61(1):103-14. Available from: http://essays.biochemistry.org/lookup/doi/10.1042/EBC20160077

42. Ruddaraju LK, Pammi SVN, Guntuku G sankar, Padavala VS, Kolapalli VRM. A review on anti-bacterials to combat resistance: From ancient era of plants and metals to present and future perspectives of green nano technological combinations. Asian J Pharm Sci. 2020;15(1):42-59.

43. Zou L, Lu J, Wang J, Ren X, Zhang L, Gao Y, et al. Synergistic antibacterial effect of silver and ebselen against multidrug-resistant Gram-negative bacterial infections. EMBO Mol Med [Internet]. 2017;9(8):1165-78. Available from: 
http://embomolmed.embopress.org/lookup/doi/10.15252/emmm.201707661

44. Salomoni R, Léo P, Montemor AF, Rinaldi BG, Rodrigues MFA. Antibacterial effect of silver nanoparticles in Pseudomonas aeruginosa. Nanotechnol Sci Appl. 2017;10:115-21.

45. Mijnendonckx K, Leys N, Mahillon J, Silver S, Van Houdt R. Antimicrobial silver: Uses, toxicity and potential for resistance. BioMetals. 2013;26(4):609-21.

46. Hwang I sok, Hwang JH, Choi H, Kim KJ, Lee DG. Synergistic effects between silver nanoparticles and antibiotics and the mechanisms involved. J Med Microbiol [Internet]. 2012 Dec 1 [cited 2019 Jan 24];61(PART12):1719-26. Available from: http://jmm.microbiologyresearch.org/content/journal/jmm/10.1099/jmm.0.04 7100-0

47. Percival SL, Bowler PG, Russell D. Bacterial resistance to silver in wound care. J Hosp Infect. 2005;60(1):1-7.

48. Medici S, Peana M, Nurchi VM, Zoroddu MA. Medical Uses of Silver: History, Myths, and Scientific Evidence. J Med Chem. 2019;62(13):5923-43.

49. Woo KJ, Hye CK, Ki WK, Shin S, So HK, Yong HP. Antibacterial activity and mechanism of action of the silver ion in Staphylococcus aureus and Escherichia coli. Appl Environ Microbiol. 2008;74(7):2171-8.

50. Morones-Ramirez JR, Winkler JA, Spina CS, Collins JJ. Silver Enhances Antibiotec Activity Against Gram-Negative Bacteria. Sci Transl Med. 2013;5(190):1-11.

51. Herisse M, Duverger Y, Martin-Verstraete I, Barras F, Ezraty B. Silver potentiates aminoglycoside toxicity by enhancing their uptake. Mol Microbiol. 2017;105(1):115-26. 
52. Randall CP, Gupta A, Jackson N, Busse D, O’Neill AJ. Silver resistance in Gramnegative bacteria: A dissection of endogenous and exogenous mechanisms. J Antimicrob Chemother. 2014;70(4):1037-46.

53. Ahmad I, Ahmad S, Editors KPR. Antibacterial Drug Discovery to Combat MDR. Antibacterial Drug Discovery to Combat MDR. 2019.

54. Kwon HB, Lee JH, Lee SH, Lee AY, Choi JS, Ahn YS. A case of argyria following colloidal silver ingestion. Ann Dermatol. 2009;21(3):308-10.

55. Day HA, Huguin C, Waller ZAE. Silver cations fold i-motif at neutral pH. Chem Commun. 2013;49(70):7696-8.

56. Zeraati M, Langley DB, Schofield P, Moye AL, Rouet R, Hughes WE, et al. I-motif DNA structures are formed in the nuclei of human cells. Nat Chem [Internet]. 2018;10(6):631-7. Available from: http://dx.doi.org/10.1038/s41557-018-00463

57. Assi HA, Garavís M, González C, Damha MJ. I-motif DNA: Structural features and significance to cell biology. Nucleic Acids Res. 2018;46(16):8038-56.

58. Jin KS, Shin SR, Ahn B, Rho Y, Kim SJ, Ree M. pH-dependent structures of an i-motif DNA in solution. J Phys Chem B. 2009;113(7):1852-6.

59. Abdelhamid MAS, Fábián L, Macdonald CJ, Cheesman MR, Gates AJ, Waller ZAE. Redox-dependent control of i-Motif DNA structure using copper cations. Nucleic Acids Res. 2018;46(12):5886-93.

60. Cheng E, Xing Y, Chen P, Yang, Sun Y, Zhou D, et al. A pH-triggered, fastresponding DNA hydrogel. Angew Chemie - Int Ed. 2009;48(41):7660-3. 
61. Takahashi S, Brazier JA, Sugimoto N. Topological impact of noncanonical DNA structures on Klenow fragment of DNA polymerase. Proc Natl Acad Sci U S A. 2017;114(36):9605-10.

62. Mergny J, Li J, Lacroix L, Amrane S, Chaires JB. Thermal difference spectra : a specific signature for nucleic acid structures Thermal difference spectra : a specific signature for nucleic acid structures. Nucleic Acids Research. 2005.

63. Surana S, Bhat JM, Koushika SP, Krishnan Y. An autonomous DNA nanomachine maps spatiotemporal $\mathrm{pH}$ changes in a multicellular living organism. Nat Commun. 2011;2(1):1-7.

64. Wang C, Du Y, Wu Q, Xuan S, Zhou J, Song J, et al. Stimuli-responsive plasmonic core-satellite assemblies: I-motif DNA linker enabled intracellular pH sensing. Chem Commun. 2013;49(51):5739-41.

65. Wright EP, Huppert JL, Waller ZAE. Identification of multiple genomic DNA sequences which form i-motif structures at neutral pH. Vol. 45, Nucleic Acids Research. 2016. p. 2951-9.

66. Day HA, Huguin C, Waller ZAE. Silver cations fold i-motif at neutral pH. Chem Commun. 2013;49(70):7696-8.

67. Li T, He N, Wang J, Li S, Deng Y, Wang Z. Effects of the i-motif DNA loop on the fluorescence of silver nanoclusters. RSC Adv. 2016;6(27):22839-44.

68. Javani S, Lorca R, Latorre A, Flors C, Cortajarena AL, Somoza Á. Antibacterial Activity of DNA-Stabilized Silver Nanoclusters Tuned by Oligonucleotide Sequence. ACS Appl Mater Interfaces. 2016;8(16):10147-54.

69. Swasey SM, Leal LE, Lopez-Acevedo O, Pavlovich J, Gwinn EG. Silver (I) as DNA 
glue: Ag+-mediated guanine pairing revealed by removing Watson-Crick constraints. Sci Rep. 2015;5(April):1-9.

70. Tewari R, Chopra D, Wazahat R, Dhingra S, Dudeja M. Antimicrobial susceptibility patterns of an emerging multidrug resistant nosocomial pathogen: Acinetobacter baumannii. Malaysian J Med Sci. 2018;25(3):129-34.

71. Owlia P, Nosrati R, Alaghehbandan R, Lari AR. Antimicrobial susceptibility differences among mucoid and non-mucoid Pseudomonas aeruginosa isolates. GMS Hyg Infect Control. 2014;9(2):Doc13.

72. Liu Y, Li R, Xiao X, Wang Z. Antibiotic adjuvants: an alternative approach to overcome multi-drug resistant Gram-negative bacteria. Crit Rev Microbiol [Internet]. 2019;45(3):301-14. Available from: https://doi.org/10.1080/1040841X.2019.1599813

73. Hobman JL, Crossman LC. Bacterial antimicrobial metal ion resistance. J Med Microbiol [Internet]. 2015 May 1 [cited 2019 Jan 23];64(5):471-97. Available from:

http://www.microbiologyresearch.org/content/journal/jmm/10.1099/jmm.0.02 $3036-0$

74. Poole K, Tetro K, Zhao Q, Neshat S, Heinrichs DE, Bianco N. Expression of the multidrug resistance operon mexA-mexB-oprM in Pseudomonas aeruginosa: mexR encodes a regulator of operon expression. Antimicrob Agents Chemother. 1996;40(9):2021-8.

75. Elkins XLCA, Editors HIZ. Effl ux-Mediated Antimicrobial Resistance in Bacteria.

76. Ganderton L, Chawla J, Winters C, Wimpenny J, Stickler D. Scanning electron 
microscopy of bacterial biofilms on indwelling bladder catheters. Eur J Clin Microbiol Infect Dis. 1992;11(9):789-96.

77. Khatoon Z, McTiernan CD, Suuronen EJ, Mah TF, Alarcon EI. Bacterial biofilm formation on implantable devices and approaches to its treatment and prevention. Heliyon [Internet]. 2018;4(12):e01067. Available from: https://doi.org/10.1016/j.heliyon.2018.e01067

78. Tsai CJY, Loh JMS, Proft T. Galleria mellonella infection models for the study of bacterial diseases and for antimicrobial drug testing. Virulence [Internet]. 2016;7(3):214-29. Available from:

http://dx.doi.org/10.1080/21505594.2015.1135289

79. Bjarnsholt T, Kirketerp-Møller K, Kristiansen S, Phipps R, Nielsen AK, Jensen PØ, et al. Silver against Pseudomonas aeruginosa biofilms. Apmis. 2007;115(8):9218.

80. Chambers JR, Cherny KE, Sauer K. Susceptibility of Pseudomonas aeruginosa dispersed cells to antimicrobial agents is dependent on the dispersion cue and class of the antimicrobial agent used. Antimicrob Agents Chemother. 2017;61(12):1-18.

81. Chua SL, Liu Y, Yam JKH, Chen Y, Vejborg RM, Tan BGC, et al. Dispersed cells represent a distinct stage in the transition from bacterial biofilm to planktonic lifestyles. Nat Commun. 2014;5.

82. Moyano AJ, Mas CR, Colque CA, Smania AM. Dealing with biofilms of Pseudomonas aeruginosa and Staphylococcus aureus: In vitro evaluation of a novel aerosol formulation of silver sulfadiazine. Burns [Internet]. 2020;46(1):128-35. Available 
from: https://doi.org/10.1016/j.burns.2019.07.027

83. Theophel K, Schacht VJ, Schlüter M, Schnell S, Stingu CS, Schaumann R, et al. The importance of growth kinetic analysis in determining bacterial susceptibility against antibiotics and silver nanoparticles. Front Microbiol. 2014;5(NOV):1-10.

84. Hoffman LR, D’Argenio DA, MacCoss MJ, Zhang Z, Jones RA, Miller SI. Aminoglycoside antibiotics induce bacterial biofilm formation. Nature. 2005;436(7054):1171-5.

85. Song T, Duperthuy M, Wai SN. Sub-optimal treatment of bacterial biofilms. Antibiotics. 2016;5(2):1-18.

86. Fish DN, Choi MK, Jung R. Synergic activity of cephalosporins plus fluoroquinolones against Pseudomonas aeruginosa with resistance to one or both drugs. J Antimicrob Chemother. 2002;50(6):1045-9.

87. Zou L, Wang J, Gao Y, Ren X, Rottenberg ME, Lu J, et al. Synergistic antibacterial activity of silver with antibiotics correlating with the upregulation of the ROS production. Sci Rep [Internet]. 2018;8(1):11131. Available from: http://www.nature.com/articles/s41598-018-29313-w

88. Tallarida RJ. Drug Synergism : Its Detection and Applications. 2001;298(3):86572.

89. Hill L, Veli N, Coote PJ. Evaluation of Galleria mellonella larvae for measuring the efficacy and pharmacokinetics of antibiotic therapies against Pseudomonas aeruginosa infection. Int J Antimicrob Agents [Internet]. 2014;43(3):254-61. Available from: http://dx.doi.org/10.1016/j.ijantimicag.2013.11.001

90. Peleg AY, Jara S, Monga D, Eliopoulos GM, Moellering RC, Mylonakis E. Galleria 
mellonella as a model system to study Acinetobacter baumannii pathogenesis and therapeutics. Antimicrob Agents Chemother. 2009;53(6):2605-9.

91. Mukherjee K, Altincicek B, Hain T, Domann E, Vilcinskas A, Chakraborty T. Galleria mellonella as a model system for studying Listeria pathogenesis. Appl Environ Microbiol. 2010;76(1):310-7.

92. Guzman CA, Rohde M, Chakraborty T, Domann E, Hudel M, Wehland J, et al. Interaction of Listeria monocytogenes with mouse dendritic cells. Infect Immun. 1995;63(9):3665-73.

93. Yang HF, Pan AJ, Hu LF, Liu YY, Cheng J, Ye Y, et al. Galleria mellonella as an in vivo model for assessing the efficacy of antimicrobial agents against Enterobacter cloacae infection. J Microbiol Immunol Infect [Internet]. 2017;50(1):55-61. Available from: http://dx.doi.org/10.1016/j.jmii.2014.11.011

94. Ignasiak K, Maxwell A. Galleria mellonella (greater wax moth) larvae as a model for antibiotic susceptibility testing and acute toxicity trials. BMC Res Notes. 2017;10(1):1-8.

95. Dean SN, Bishop BM, Van Hoek ML. Susceptibility of Pseudomonas aeruginosa biofilm to alpha-helical peptides: D-enantiomer of LL-37. Front Microbiol. 2011;2(JULY):1-11.

96. Thomas RJ, Hamblin KA, Armstrong SJ, Müller CM, Bokori-Brown M, Goldman S, et al. Galleria mellonella as a model system to test the pharmacokinetics and efficacy of antibiotics against Burkholderia pseudomallei. Int J Antimicrob Agents [Internet]. 2013;41(4):330-6. Available from: http://dx.doi.org/10.1016/j.ijantimicag.2012.12.009 
97. Rai M, Yadav A, Gade A. Silver nanoparticles as a new generation of antimicrobials. Biotechnol Adv [Internet]. 2009;27(1):76-83. Available from: http://dx.doi.org/10.1016/j.biotechadv.2008.09.002

98. Li WR, Sun TL, Zhou SL, Ma YK, Shi QS, Xie XB, et al. A comparative analysis of antibacterial activity, dynamics, and effects of silver ions and silver nanoparticles against four bacterial strains. Int Biodeterior Biodegrad [Internet]. 2017;123:304-10. Available from: http://dx.doi.org/10.1016/j.ibiod.2017.07.015

99. Atiyeh BS, Costagliola M, Hayek SN, Dibo SA. Effect of silver on burn wound infection control and healing: Review of the literature. Vol. 33, Burns. 2007. p. 139-48.

100. Miquel S, Lagrafeuille R, Souweine B, Forestier C. Anti-biofilm activity as a health issue. Front Microbiol. 2016;7(APR):1-14.

101. Tré-Hardy M, Nagant C, El Manssouri N, Vanderbist F, Traore H, Vaneechoutte M, et al. Efficacy of the combination of tobramycin and a macrolide in an in vitro Pseudomonas aeruginosa mature biofilm model. Antimicrob Agents Chemother. 2010;54(10):4409-15.

102. Erzsele ÁKOSJ, Ere ERPÁ. EVALUATING SYNERGY BETWEEN MARBOFLOXACIN AND GENTAMICIN IN PSEUDOMONAS AERUGINOSA STRAINS ISOLATED FROM DOGS WITH OTITIS EXTERNA. 2015;62(1):45-55.

103. Pamp SJ, Gjermansen M, Johansen HK, Tolker-Nielsen T. Tolerance to the antimicrobial peptide colistin in Pseudomonas aeruginosa biofilms is linked to metabolically active cells, and depends on the pmr and mexAB-oprM genes. Mol Microbiol. 2008;68(1):223-40. 
104. Fantin B, Carbon C. In vivo antibiotic synergism: Contribution of animal models. Antimicrob Agents Chemother. 1992;36(5):907-12.

105. Jarrell AS, Kruer RM, Johnson D, Lipsett PA. Antimicrobial Pharmacokinetics and Pharmacodynamics. Surg Infect (Larchmt). 2015;16(4):375-9.

106. Kaegi R, Voegelin A, Ort C, Sinnet B, Thalmann B, Krismer J, et al. Fate and transformation of silver nanoparticles in urban wastewater systems. Water Res [Internet]. 2013;47(12):3866-77. Available from: http://dx.doi.org/10.1016/j.watres.2012.11.060

107. Cannon CL, Hogue LA, Vajravelu RK, Capps GH, Ibricevic A, Hindi KM, et al. In vitro and murine efficacy and toxicity studies of nebulized SCC1, a methylated caffeinesilver(I) complex, for treatment of pulmonary infections. Antimicrob Agents Chemother. 2009;53(8):3285-93.

108. Doherty MM, Pang KS. First-pass effect: Significance of the intestine for absorption and metabolism. Drug Chem Toxicol. 1997;20(4):329-44.

109. Guo W, Qi XJ, Orbach R, Lu CH, Freage L, Mironi-Harpaz I, et al. Reversible Ag+crosslinked DNA hydrogels. Chem Commun. 2014;50(31):4065-8.

110. Drobnic ME, Suñé P, Montoro JB, Ferrer A, Orriols R. Inhaled tobramycin in noncystic fibrosis patients with bronchiectasis and chronic bronchial infection with Pseudomonas aeruginosa. Ann Pharmacother. 2005;39(1):39-44.

111. Wiemann M, Vennemann A, Blaske F, Sperling M, Karst U. Silver nanoparticles in the lung: Toxic effects and focal accumulation of silver in remote organs. Nanomaterials. 2017;7(12):1-26.

112. Yuan Z, Chen YC, Li HW, Chang HT. Fluorescent silver nanoclusters stabilized by 
DNA scaffolds. Chem Commun. 2014;50(69):9800-15.

113. Wright EP, Day HA, Ibrahim AM, Kumar J, Boswell LJE, Huguin C, et al. Mitoxantrone and analogues bind and stabilize i-motif forming DNA sequences. Sci Rep. 2016;6:4-10.

114. Ali MM, Li F, Zhang Z, Zhang K, Kang DK, Ankrum JA, et al. Rolling circle amplification: A versatile tool for chemical biology, materials science and medicine. Chem Soc Rev. 2014;43(10):3324-41.

115. Zhao W, Ali MM, Brook MA, Li Y. Rolling circle amplification: Applications in nanotechnology and biodetection with functional nucleic acids. Angew Chemie Int Ed. 2008;47(34):6330-7.

116. Bader MS. Diabetic foot infection. Am Fam Physician. 2008;78(1).

117. Alejandro Almonaci Hernández C, Juarez-Moreno K, Castañeda-Juarez ME, Almanza-Reyes H, Pestryakov A, Bogdanchikova N, et al. Silver Nanoparticles for the Rapid Healing of Diabetic Foot Ulcers. Int J Med Nano Res. 2017;4(1):19.

118. Davies JC. Pseudomonas aeruginosa in cystic fibrosis: Pathogenesis and persistence. Paediatr Respir Rev. 2002;

119. Takenaka S, Karg E, Roth C, Schulz H, Ziesenis A, Heinzmann U, et al. Pulmonary and systemic distribution of inhaled ultrafine silver particles in rats. Environ Health Perspect. 2001;109(SUPPL. 4):547-51. 



\section{APPENDICES}

\section{Appendix A. Buffers and solutions}

All buffers and solutions used in this study were prepared with $\mathrm{ddH}_{2} \mathrm{O}$. The $\mathrm{pH}$ adjustments were made using a calibrated pH meter (Orion, Model SA520).

AgiM Master Mix variations

6:1 $\mathrm{Ag}^{+}$: DNA ratio Master Mix

100 mM MES pH $6.5 \quad 40 \mu \mathrm{L}$

$250 \mu \mathrm{M}$ iMotif oligonucleotide $\quad 20 \mu \mathrm{L}$

$\mathrm{H}_{2} \mathrm{O}$ MilliQ $\quad 310 \mu \mathrm{L}$

$1 \mathrm{mM} \mathrm{AgNO}_{3} \quad 30 \mu \mathrm{L}$

$\underline{10: 1 \mathrm{Ag}^{+} \text {: DNA ratio Master Mix }}$

100 mM MES pH $6.5 \quad 40 \mu \mathrm{L}$

$250 \mu \mathrm{M}$ iMotif oligonucleotide $\quad 20 \mu \mathrm{L}$

$\mathrm{H}_{2} \mathrm{O}$ MilliQ $\quad 290 \mu \mathrm{L}$

$1 \mathrm{mM} \mathrm{AgNO}_{3} \quad 50 \mu \mathrm{L}$

20:1 $\mathrm{Ag}^{+}$: DNA ratio Master Mix

100 mM MES pH $6.5 \quad 40 \mu \mathrm{L}$

$250 \mu \mathrm{M}$ iMotif oligonucleotide $\quad 20 \mu \mathrm{L}$

$\mathrm{H}_{2} \mathrm{O}$ MilliQ $\quad 240 \mu \mathrm{L}$

$1 \mathrm{mM} \mathrm{AgNO}_{3} \quad 100 \mu \mathrm{L}$ 


\section{1 x Tris-EDTA (TE) (pH 8.0)}

1 M Tris base $\quad 10 \mathrm{~mL}$

$0.5 \mathrm{~m} \mathrm{Na} 2 \mathrm{EDTA}$

Compounds dissolved in $800 \mathrm{~mL}$ ddH20, then $\mathrm{pH}$ adjusted to 8.0 and volume adjusted to $1 \mathrm{~L}$ before autoclaving.

\section{0 x Phosphate Buffered Solution (PBS) (pH 7.4)}

$\mathrm{NaCl} \quad 80 \mathrm{~g} / \mathrm{L}$

$\mathrm{Na}_{2} \mathrm{HPO}_{4} \quad 14.2 \mathrm{~g} / \mathrm{L}$

$\mathrm{KCl} \quad 2 \mathrm{~g} / \mathrm{L}$

$\mathrm{KH}_{2} \mathrm{PO}_{4}$

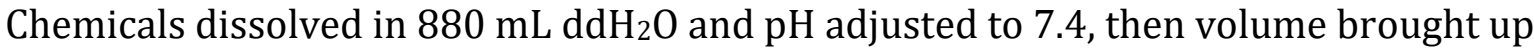
to $1 \mathrm{~L}$ before autoclaving at $121^{\circ} \mathrm{C}$ for $20 \mathrm{~min}$. $1 \mathrm{X}$ PBS made by dilutiong $1: 10$ in $\mathrm{dd}_{2} \mathrm{O}$, and autoclaving before use. 


\section{Appendix B. Susceptibilities of bacterial strains}

Susceptibility to antibiotics was determined using the Kirby-Bauer Disk Diffusion Protocol to determine the sensitivity or resistance of the bacterial strains to a variety of antibiotics. Growth inhibition zones are measured to determine a classification of $\mathrm{S}=$ sensitive; I = intermediate (Sensitive/Resistant) or $\mathrm{R}$ = resistant.

All tests were performed in duplicate using both Lysogeny Broth (LB) nutrient media and Mueller Hinton (MH) nutrient media. The Kirby-Bauer Disk Diffusion Protocol and CLSI instruct the use of MH nutrient medium as the gold standard for susceptibility testing. It is a non-selective, non-differential medium with proven reproducibility for susceptibility testing. LB nutrient medium was also used in this test protocol as it is the chosen growth medium used throughout this study. A microbiology standard, LB media is the most widely used nutrient-rich medium for the culture and growth of bacteria. Figure 1 and Figure 2 show the agar plates for $P$. aeruginosa and $A$. baumannii, respectively; with $\mathrm{MH}$ agar plates on the left and LB agar plates on the right. These figures confer LB and MH nutrient mediums produce comparable growth and inhibition zones for all strains of bacteria tested. 

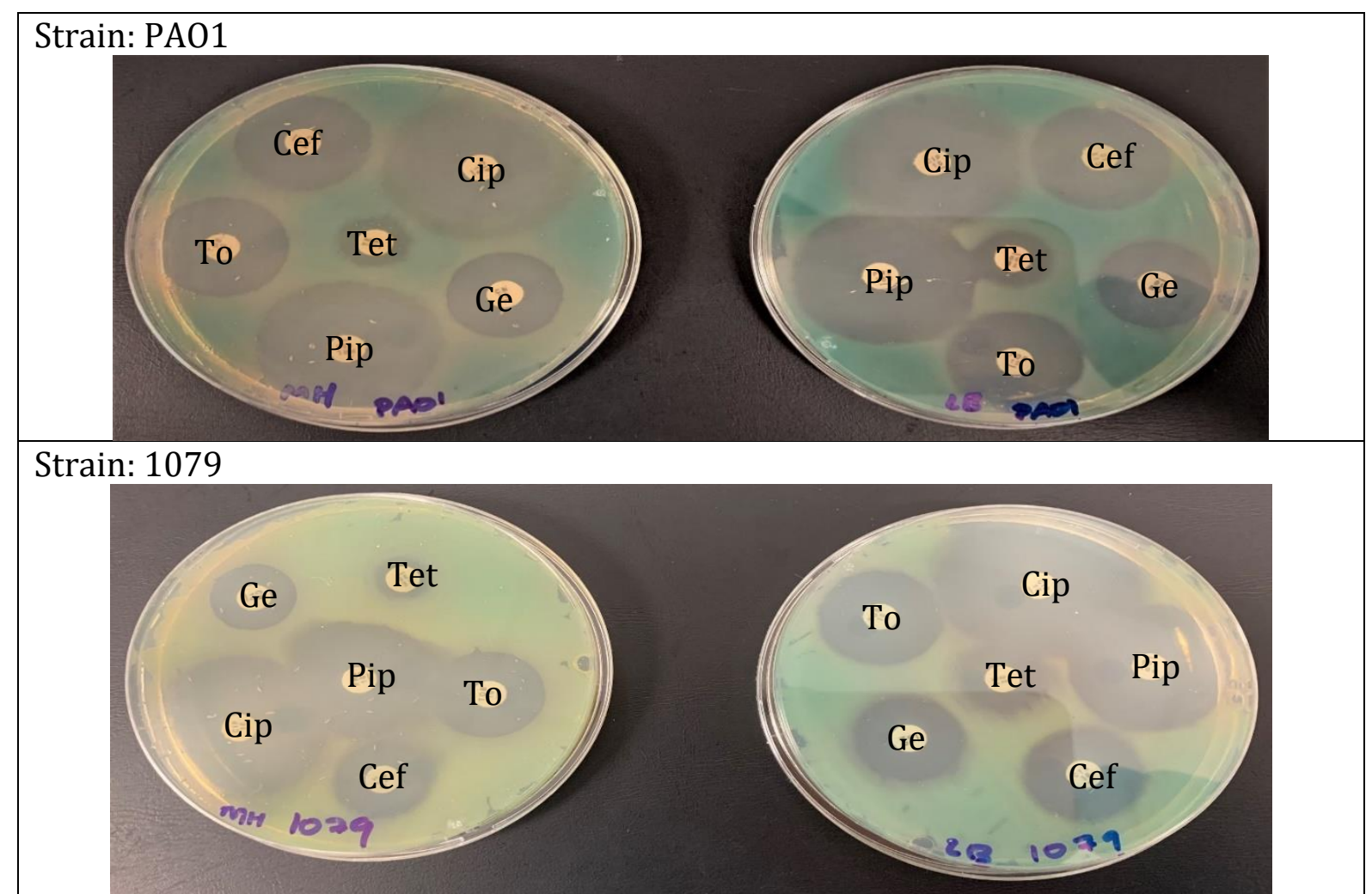

Strain: 1024

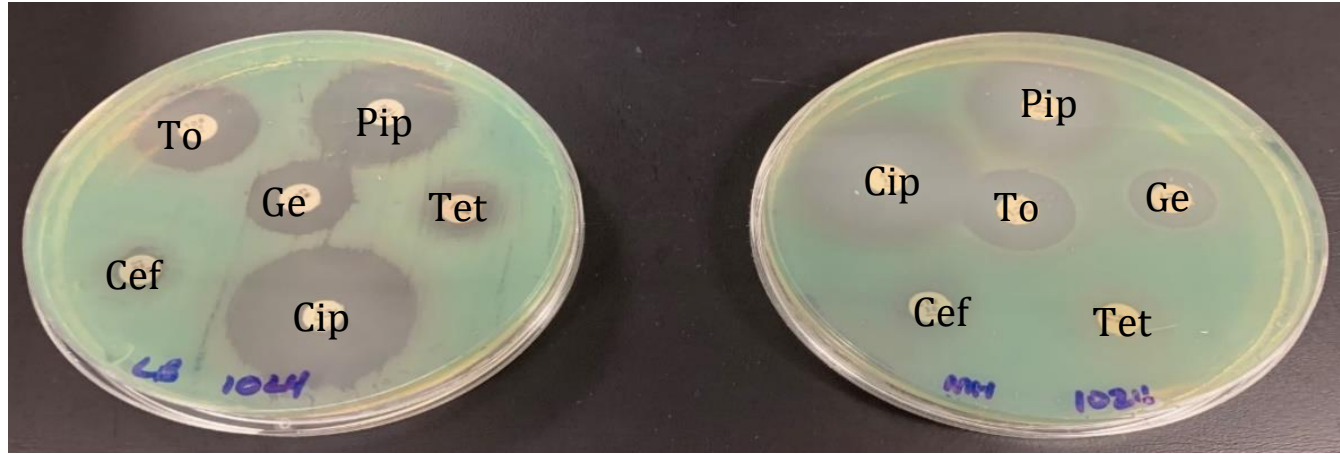

Strain: 1205

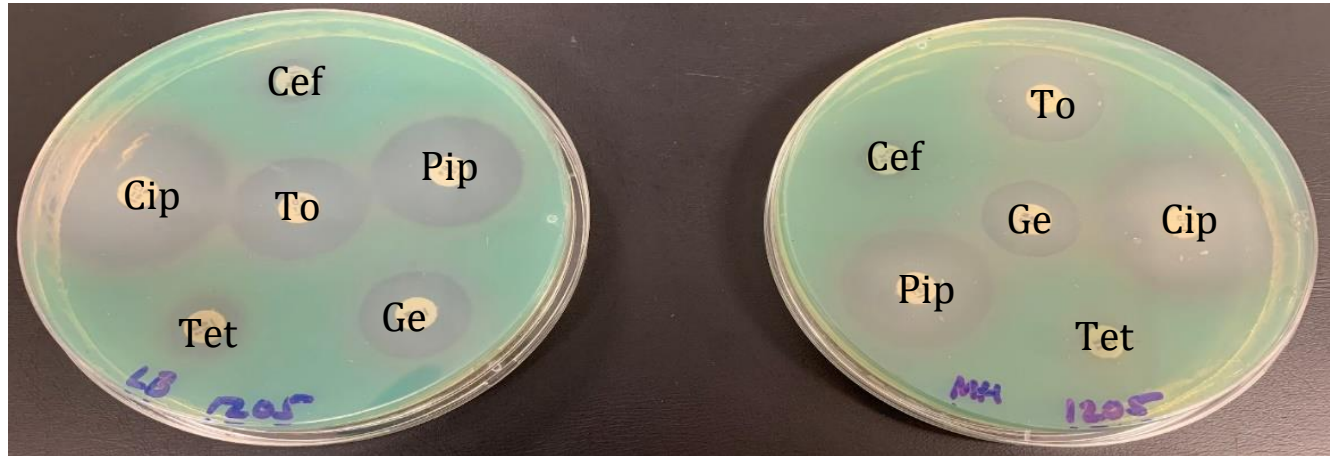

Susceptibility to Antimicrobials of PAO1 and clinical isolates. Antibiotics tested Tobramycin (Tob)., Ciprofloxacin (Cip), Piperacillin (Pip), Cefotaxime (Cef), Tetracycline (Tet), Gentamicin (Gen). Plates on the left are MH agar compared with LB agar on the right. 

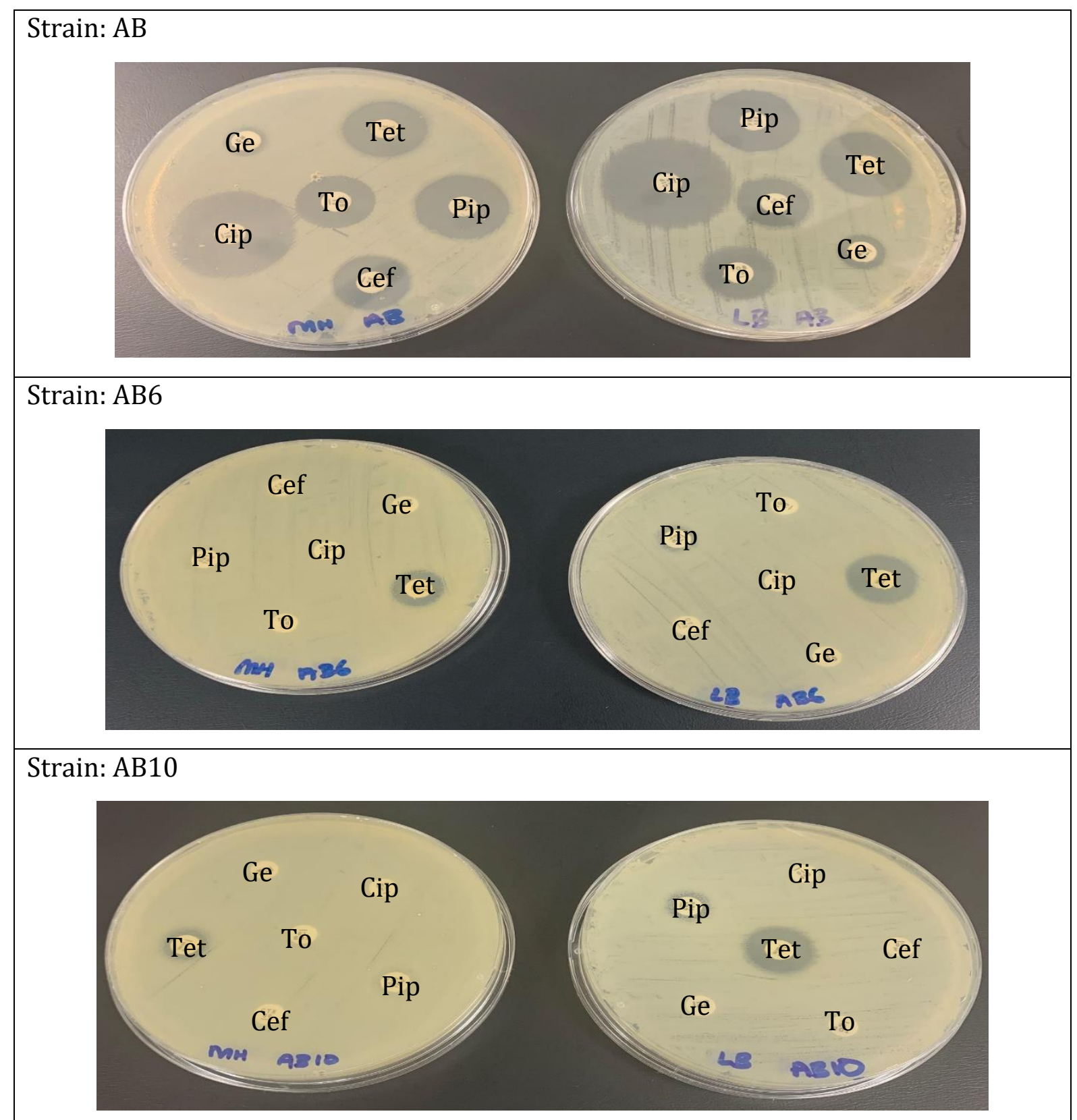

Strain: AB12

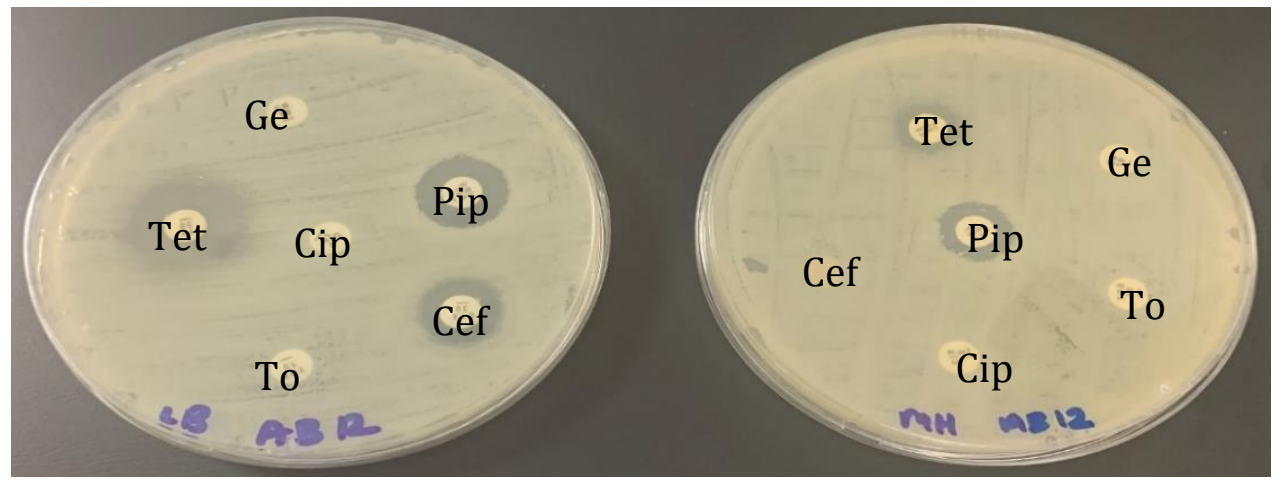

Susceptibility to Antimicrobials of AB and clinical isolates. Antibiotics tested Tobramycin (Tob)., Ciprofloxacin (Cip), Piperacillin (Pip), Cefotaxime (Cef), Tetracycline (Tet), Gentamicin (Gen). Plates on the left are MH agar compared with LB agar on the righ 
